\title{
Substrate-Controlled Asymmetric Total Synthesis and Structure Revision of (-)-Bisezakyne A
}

\author{
Iljin Shin, Dongjoo Lee, *Hyoungsu Kim*
}

College of Pharmacy and Research Institute of Pharmaceutical Science and Technology (RIPST), Ajou University, Suwon 16499, Republic of Korea

*E-mail: dongjoo@ ajou.ac.kr

*E-mail: hkimajou@ajou.ac.kr

Supporting Information (Part A): Experimental Procedures.....S1 S29

Supporting Information (Part B): NMR Spectra................S30 S149 


\title{
- Supporting Information: Part A -
}

\author{
Part A (S3 S29)
}

Experimental Procedures and Product Characterizations

General \& Note: S3

Asymmetric Synthesis of Proposed and Revised Structure of Bisezakyne A: S4 S27

Synthesis of Key Common 6,7-syn-Hydroxy Bromo Amide 4: S3 S6

Synthesis of 9,10-anti-Intramolecular Amide Enolate Alkylation (IAEA) Substrates: S8 S9

IAEA of 9,10-anti-10-TIPS or PMB-protectedSubstrates: S9 S11

Synthesis of Authentic 9,12-cis- \& 9,12-trans-IAEA Products: S12 S14

Synthesis of Proposed Structure of Bisezakyne A (1a): S15 S22

Synthesis of Revised Structure of Bisezakyne A (1b): S23 S27

References: S27

Comparative ${ }^{1} \mathrm{H} \&{ }^{13} \mathrm{C}$ NMR Data for Bisezakyne A: S28 S29 


\section{General \& Note : S3}

\section{General}

Proton $\left({ }^{1} \mathrm{H}\right)$ and carbon $\left({ }^{13} \mathrm{C}\right)$ NMR spectra were obtained on a Varian Mercury 400, Bruker Avance II 400, Unity Inova 500, Bruker Avance III 500, or Avance III 700 spectrometer. Chemical shifts are reported in ppm units with $\mathrm{Me}_{4} \mathrm{Si}$ or $\mathrm{CHCl}_{3}$ as the internal standard. Specific rotation was obtained on a Jasco P-2000 (light source, WI $589 \mathrm{~nm}$ ). High resolution mass spectra (HRMS) were recorded using electronionization (EI) and chemical ionization (CI). Melting point was obtained on an IA9100. All reactions were routinely carried out under an inert atmosphere of dry nitrogen. Reactions were checked by thin layer chromatography (Kieselgel 60 F254, Merck). Spots were detected by viewing under a UV light, and by colorizing with charring after dipping in $p$-anisaldehyde solution in a mixture of acetic acid, sulfuric acid, and methanol. In aqueous work-up, all organic solutions were dried over anhydrous sodium sulfate and filtered prior to rotary evaporation. The crude compounds were purified by column chromatography on a silica gel (Kieselgel 60, 70-230 mesh, Merck). Unless otherwise noted, materials and all solvents were obtained from commercial suppliers and were used without purification. Toluene and methylene chloride were dried with $4 \AA$ molecular sieve.THF was freshly distilled from sodium and benzophenone.

Note: The present Supporting Information A includes modified versions of the experimental procedures that have been already described in theliterature in casethe experimental procedures were improved in terms of reaction conditions, yields, selectivity, and so on, or where additional spectroscopic data are available. 
Asymmetric Total Synthesis of Proposed and Revised Structures

$$
\text { of }
$$

Bisezakyne A 1a \& 1b:

$$
\text { S4 S27 }
$$




\section{Synthesis of Key Common 6,7-syn-Hydroxy Bromo Amide 4: S3 S6}

\section{Determination of Enantiomeric Excess of Epoxy Alcohol 7 by the Mosher Method ${ }^{2}$ Preparation of (S)-MTPA Ester SI-A-1}
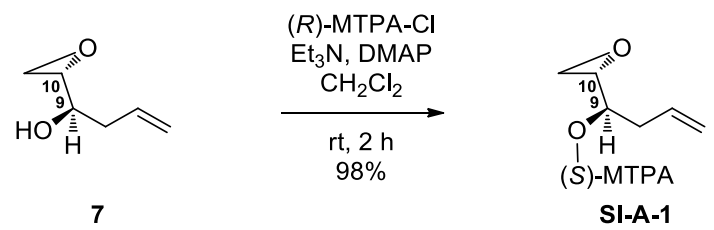

SI-A-1

To a solution of known epoxy alcohol $7^{1}(6.0 \mathrm{mg}, 0.05 \mathrm{mmol})$ in $\mathrm{CH}_{2} \mathrm{Cl}_{2}$ were successively added $(R)-(+)-\alpha$-methoxy- $\alpha$-trifluoromethylphenylacetyl chloride $(0.020 \mathrm{~mL}, 0.11 \mathrm{mmol}), \mathrm{Et}_{3} \mathrm{~N}$ $(0.015 \mathrm{~mL}, 0.11 \mathrm{mmol})$, and DMAP $(10.0 \mathrm{mg}, 0.08 \mathrm{mmol})$ at room temperature. After being stirred for $2 \mathrm{~h}$ at the same temperature, the reaction mixture was quenched with saturated aqueous $\mathrm{NaHCO}_{3}$ and diluted with $\mathrm{Et}_{2} \mathrm{O}$. The layers were separated, and the aqueous layer was extracted with $\mathrm{Et}_{2} \mathrm{O}$. The combined organic layers were washed with saturated brine, dried over anhydrous $\mathrm{Na}_{2} \mathrm{SO}_{4}$, filtered, and concentrated in vacuo. The residue was filtered through a short column of silica gel (hexanes/ethyl acetate, 5:1) to afford the crude (S)-MTPA Ester SI-A-1 as a colorless oil (17.0 mg, 98\%): ${ }^{1} \mathrm{H}$ NMR (400 $\left.\mathrm{MHz}, \mathrm{CDCl}_{3}\right) \delta 7.53-7.51$ (m, $\left.2 \mathrm{H}\right), 7.43-7.36$ (m, $3 \mathrm{H}), 5.79$ (dddd, $J=17.2,10.0,6.4,6.4 \mathrm{~Hz}, 1 \mathrm{H}$ ), 5.20-5.14 (m, $2 \mathrm{H}$ ), 5.09 (ddd, $J=7.6$, 4.8, $4.8 \mathrm{~Hz}, 1 \mathrm{H}), 3.54(\mathrm{~d}, J=1.6 \mathrm{~Hz}, 3 \mathrm{H}), 2.99$ (ddd, $J=6.4,4.0,2.4 \mathrm{~Hz}, 1 \mathrm{H}), 2.70(\mathrm{dd}, J=$ 4.0, 4.0 Hz, $1 \mathrm{H}), 2.64(\mathrm{dd}, J=5.2,2.4 \mathrm{~Hz}, 1 \mathrm{H}), 2.63-2.48(\mathrm{~m}, 2 \mathrm{H})$.

\section{Preparation of $(R)$-MTPA Ester SI-A-2}
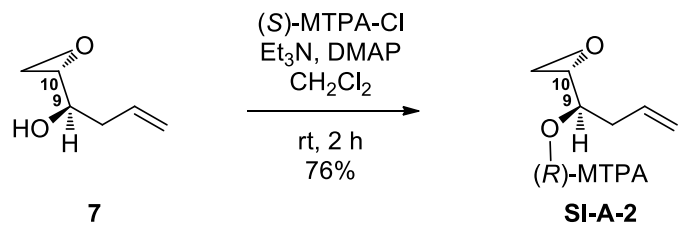

To a solution of epoxy alcohol $7(6.0 \mathrm{mg}, 0.05 \mathrm{mmol})$ in $\mathrm{CH}_{2} \mathrm{Cl}_{2}$ were successively added $(S)$ (+)- $\alpha$-methoxy- $\alpha$-trifluoromethylphenylacetyl chloride $(0.020 \mathrm{~mL}, 0.11 \mathrm{mmol}), \mathrm{Et}_{3} \mathrm{~N}(0.015 \mathrm{~mL}$, $0.11 \mathrm{mmol})$, and DMAP $(10.0 \mathrm{mg}, 0.08 \mathrm{mmol})$ at room temperature. After being stirred for $2 \mathrm{~h}$ at the same temperature, the reaction mixture was quenched with saturated aqueous $\mathrm{NaHCO}_{3}$ and diluted with $\mathrm{Et}_{2} \mathrm{O}$. The layers were separated, and the aqueous layer was extracted with $\mathrm{Et}_{2} \mathrm{O}$. 
The combined organic layers were washed with saturated brine, dried over anhydrous $\mathrm{Na}_{2} \mathrm{SO}_{4}$, filtered, and concentrated in vacuo. The residue was filtrated by column chromatography (silica gel, hexanes/ethyl acetate, 5:1) to afford the crude $(\boldsymbol{R})$-MTPA Ester SI-A-2 as a colorless oil $(13.0 \mathrm{mg}, 76 \%):{ }^{1} \mathrm{H}$ NMR $\left(400 \mathrm{MHz}, \mathrm{CDCl}_{3}\right) \delta 7.53-7.51(\mathrm{~m}, 2 \mathrm{H}), 7.42-7.36(\mathrm{~m}, 3 \mathrm{H}), 5.67$ (dddd, $J=17.2,10.0,7.2,7.2 \mathrm{~Hz}, 1 \mathrm{H}$ ), 5.15 (ddd, $J=7.2,4.8,4.8 \mathrm{~Hz}, 1 \mathrm{H}$ ), 5.10-5.05 (m, $2 \mathrm{H}$ ), $3.54(\mathrm{~d}, J=1.2 \mathrm{~Hz}, 3 \mathrm{H}), 3.10(\mathrm{ddd}, J=6.8,4.4,4.4 \mathrm{~Hz}, 1 \mathrm{H}), 2.76(\mathrm{dddd}, J=8.8,5.2,5.2,5.2$ $\mathrm{Hz}, 2 \mathrm{H}), 2.56-2.41(\mathrm{~m}, 2 \mathrm{H})$.

\section{Preparation of Epoxy Amide 8}

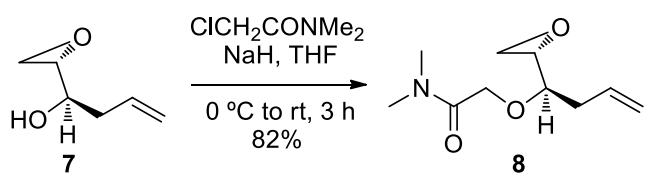

To a cooled $\left(0^{\circ} \mathrm{C}\right)$ solution of the known epoxy $7(6.38 \mathrm{~g}, 55.89 \mathrm{mmol})$ in THF $(100 \mathrm{~mL}, 0.56 \mathrm{M})$ was added $\mathrm{NaH}$ (4.47 g, 60\% dispersion in mineral oil, $111.79 \mathrm{mmol}$ ). The resulting mixture was stirred for $10 \mathrm{~min}$ at the same temperature, and 2-chloro- $N, N$-dimethylacetamide $(7.48 \mathrm{~g}, 61.47$ mmol) was added. After being stirred for $3 \mathrm{~h}$ at room temperature, the reaction mixture was quenched with saturated aqueous $\mathrm{NH}_{4} \mathrm{Cl}$ and diluted with EtOAc. The layers were separated, and the aqueous layer was extracted with EtOAc. The combined organic layers were washed with brine, dried over anhydrous $\mathrm{Na}_{2} \mathrm{SO}_{4}$, and concentrated in vacuo. The residue was purified by column chromatography (silica gel, $n$-hexane/EtOAc, $2 / 1$ to EtOAc only) to afford $\alpha$-alkoxy amide 8 as a yellow oil $(9.15 \mathrm{~g}, 82 \%):[\alpha]^{20.3}=-6.11\left(c 3.96, \mathrm{CHCl}_{3}\right) ;{ }^{1} \mathrm{H} \mathrm{NMR}(400 \mathrm{MHz}$, $\left.\mathrm{CDCl}_{3}\right) \delta 5.90(\mathrm{dddd}, J=17.2,10.0,6.8,6.8 \mathrm{~Hz}, 1 \mathrm{H}), 5.14(\mathrm{ddd}, J=17.2,6.8,1.6 \mathrm{~Hz}, 1 \mathrm{H}), 5.08$ $(\mathrm{dd}, J=9.6,1.6 \mathrm{~Hz}, 1 \mathrm{H}), 4.23\left(\mathrm{AB}, J_{\mathrm{AB}}=13.6 \mathrm{~Hz}, \Delta v_{\mathrm{AB}}=22.4 \mathrm{~Hz}, 2 \mathrm{H}\right), 3.40(\mathrm{ddd}, J=6.4,5.6$, $5.6 \mathrm{~Hz}, 1 \mathrm{H}), 3.0(\mathrm{~s}, 3 \mathrm{H}), 2.97$ (ddd, $J=6.4,3.6,2.8 \mathrm{~Hz}, 1 \mathrm{H}), 2.93$ (s, 3H), 2.82-2.78 (m, 2H), 2.47-2.43 (m, 2H); ${ }^{13} \mathrm{C}$ NMR $\left(100 \mathrm{MHz}, \mathrm{CDCl}_{3}\right) \delta 168.7,133.5,117.3,78.1,69.3,52.6,45.3$, 37.0, 36.5, 35.3; IR (neat) $1643 \mathrm{~cm}^{-1}$; HRMS (EI) found 199.1204 [calcd for $\mathrm{C}_{10} \mathrm{H}_{17} \mathrm{NO}_{3}(\mathrm{M})^{+}$ 199.1208]. 


\section{Preparation of 9,10-anti-Hydroxy Bromo Amide 9}
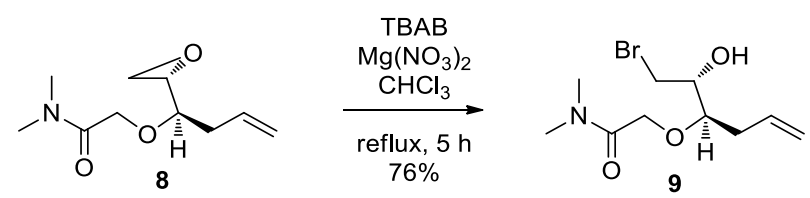

To a solution of $\alpha$-alkoxy amide $8(7.77 \mathrm{~g}, 39.0 \mathrm{mmol})$ in $\mathrm{CHCl}_{3}(100 \mathrm{~mL}, 0.4 \mathrm{M})$ were added TBAB (tetrabutylammonium bromide, $31.00 \mathrm{~g}, 97.5 \mathrm{mmol}$ ) and magenesium nitrate hexahydrate (7.00 g, $27.3 \mathrm{mmol})$ at room temperature. The resulting mixture was stirred at $90{ }^{\circ} \mathrm{C}$ for $6 \mathrm{~h}$, quenched with $\mathrm{H}_{2} \mathrm{O}$ at room temperature, and diluted with excess EtOAc. The layers were separated, and the aqueous layer was extracted with EtOAc. The combined organic layers were washed with brine, dried over anhydrous $\mathrm{Na}_{2} \mathrm{SO}_{4}$, and concentrated in vacuo. The residue was purified by column chromatography (silica gel, $n$-hexane/ethyl acetate, $1 / 1$ to 1/2) to afford hydroxy bromo amide 9 as a yellow oil $(8.32 \mathrm{~g}, 76 \%)$ : $[\alpha]^{21.8}{ }_{\mathrm{D}}=-61.47\left(c 1.37, \mathrm{CHCl}_{3}\right) ;{ }^{1} \mathrm{H}$ NMR $\left(400 \mathrm{MHz}, \mathrm{CDCl}_{3}\right) \delta 5.84(\mathrm{dddd}, J=17.2,10.4,7.2,7.2 \mathrm{~Hz}, 1 \mathrm{H}), 5.16(\mathrm{dd}, J=17.2,1.6$ $\mathrm{Hz}, 1 \mathrm{H}), 5.11(\mathrm{~d}, J=10.0 \mathrm{~Hz} 1 \mathrm{H}), 4.26\left(\mathrm{AB}, J_{\mathrm{AB}}=15.6 \mathrm{~Hz}, \Delta v_{\mathrm{AB}}=138.0 \mathrm{~Hz}, 2 \mathrm{H}\right), 3.86-3.81(\mathrm{~m}$, $1 \mathrm{H}), 3.61-3.51(\mathrm{~m}, 4 \mathrm{H}), 2.97$ (s, $3 \mathrm{H}), 2.92(\mathrm{~s}, 3 \mathrm{H}), 2.48-2.40(\mathrm{~m}, 1 \mathrm{H}), 2.36-2.30(\mathrm{~m}, 1 \mathrm{H}){ }^{13} \mathrm{C}$ NMR $\left(100 \mathrm{MHz}, \mathrm{CDCl}_{3}\right) \delta 170.1,133.8,117.8,83.7,72.5,68.1,35.77,35.71,35.59,35.0$; IR (neat) $3306,1639 \mathrm{~cm}^{-1}$; HRMS (EI) found 279.0470 [calcd for $\mathrm{C}_{10} \mathrm{H}_{18} \mathrm{BrNO}_{3}(\mathrm{M})^{+} 279.0470$ ]. 


\section{Synthesis of 9,10-anti-Intramolecular Amide Enolate Alkylation (IAEA) Substrates: S8 S9}

\section{Preparation of TIPS-protected Bromo Amide 6}

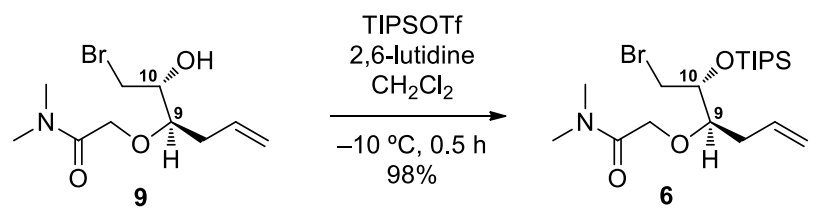

To a cooled $\left(-10{ }^{\circ} \mathrm{C}\right)$ solution of hydroxy bromo amide $9(1.21 \mathrm{~g}, 4.33 \mathrm{mmol})$ in $\mathrm{CH}_{2} \mathrm{Cl}_{2}(40 \mathrm{~mL}$, $0.1 \mathrm{M})$ were added 2,6-lutidine $(3.03 \mathrm{~g}, 25.98 \mathrm{mmol})$ and TIPSOTf $(1.16 \mathrm{~mL}, 4.33 \mathrm{mmol})$. The resulting mixture was stirred for $0.5 \mathrm{~h}$ at the same temperature, quenched with $\mathrm{MeOH}$, and diluted with saturated aqueous $\mathrm{NaHCO}_{3}$ and excess EtOAc. The layers were separated, and the aqueous layer was extracted with EtOAc. The combined organic layers were washed with brine, dried over anhydrous $\mathrm{Na}_{2} \mathrm{SO}_{4}$, and concentrated in vacuo. The residue was purified by column $(n$-hexane/ethyl acetate $=4 / 1)$ to afford TIPS-protected bromo amide $\mathbf{6}$ as a yellow oil $(1.85 \mathrm{~g}$, 98\%): $[\alpha]^{21.8}{ }_{\mathrm{D}}=+10.13\left(\right.$ c $\left.2.69, \mathrm{CHCl}_{3}\right) ;{ }^{1} \mathrm{H} \mathrm{NMR}\left(400 \mathrm{MHz}, \mathrm{CDCl}_{3}\right) \delta 5.88$ (dddd, $J=17.2$, 10.0, 7.2, 7.2 Hz, $1 \mathrm{H}), 5.13(\mathrm{dd}, J=17.2,1.2 \mathrm{~Hz}, 1 \mathrm{H}), 5.06$ (d, J=10.4 Hz, $1 \mathrm{H}), 4.27$ (s, $2 \mathrm{H})$, 4.06 (ddd, $J=8.8,4.8,4.8 \mathrm{~Hz}, 1 \mathrm{H}), 3.67-3.61$ (m, $2 \mathrm{H}$ ), 3.50 (dd, $J=10.4,5.2 \mathrm{~Hz}, 1 \mathrm{H}), 2.97$ (s, $3 \mathrm{H}), 2.92(\mathrm{~s}, 3 \mathrm{H}), 2.50-2.38(\mathrm{~m}, 2 \mathrm{H}), 1.05(\mathrm{~s}, 21 \mathrm{H}){ }^{13} \mathrm{C} \mathrm{NMR}\left(100 \mathrm{MHz}, \mathrm{CDCl}_{3}\right) \delta 168.4$, 134.6, 117.2, 81.6, 73.6, 69.8, 36.4, 35.5, 35.3, 35.1, 18.1, 12.7; IR (neat) 1644, 752 $\mathrm{cm}^{-1}$; HRMS (EI) found 435.1810 [calcd for $\mathrm{C}_{19} \mathrm{H}_{38} \mathrm{BrNO}_{3} \mathrm{Si}(\mathrm{M})^{+} 435.1804$ ].

\section{Preparation of PMB-protected Bromo Amide 10}
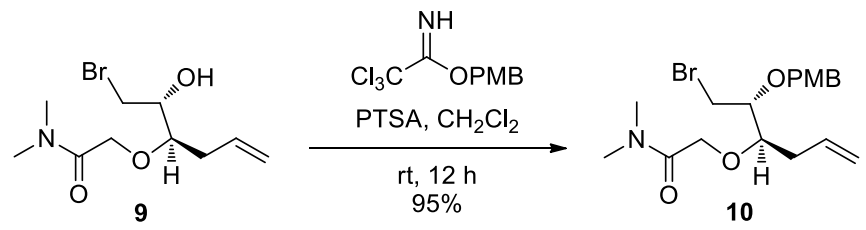

To a cooled $\left(0^{\circ} \mathrm{C}\right)$ solution of hydroxy bromo amide 9 (111.3 g, $\left.0.40 \mathrm{mmol}\right)$ in $\mathrm{CH}_{2} \mathrm{Cl}_{2}(4 \mathrm{~mL}$, $0.1 \mathrm{M})$ were successively added 4-methoxybenzyl 2,2,2-trichloroacetimidate $(0.25 \mathrm{~mL}, 1.20$ 
mmol) and PTSA (15.1 mg, $0.08 \mathrm{mmol})$. After being stirred for $12 \mathrm{~h}$ at room temperature, the resulting mixture was quenched with triethylamine, and concentrated in vacuo. The residue was purified by column chromatography (hexanes/ethyl acetate, 1:1) to afford PMB-protected bromo amide 10 as a colorless oil(127.8mg, 95\%): $[\alpha]^{21}{ }_{\mathrm{D}}=-2.4\left(c 1.00, \mathrm{CHCl}_{3}\right) ;{ }^{1} \mathrm{H} \mathrm{NMR}(400 \mathrm{MHz}$, $\left.\mathrm{CDCl}_{3}\right) \delta 7.28(\mathrm{~d}, J=8.8 \mathrm{~Hz}, 2 \mathrm{H}), 6.87(\mathrm{~d}, J=8.8 \mathrm{~Hz}, 2 \mathrm{H}), 5.84(\mathrm{dddd}, J=17.2,10.4,7.2,7.2$ $\mathrm{Hz}, 1 \mathrm{H}), 5.11(\mathrm{~d}, J=17.2 \mathrm{~Hz}, 1 \mathrm{H}), 5.08(\mathrm{~d}, J=10.4 \mathrm{~Hz}, 1 \mathrm{H}), 4.55\left(\mathrm{AB}, J_{\mathrm{AB}}=11.2 \mathrm{~Hz}, \Delta v_{\mathrm{AB}}=\right.$ $59.6 \mathrm{~Hz}, 2 \mathrm{H}), 4.24\left(\mathrm{AB}, J_{\mathrm{AB}}=13.2 \mathrm{~Hz}, \Delta v_{\mathrm{AB}}=35.2 \mathrm{~Hz}, 2 \mathrm{H}\right), 3.80(\mathrm{~s}, 3 \mathrm{H}), 3.73-3.61(\mathrm{~m}, 3 \mathrm{H})$, 2.95 (s, $3 \mathrm{H}), 2.92$ (s, $3 \mathrm{H}), 2.51-2.39(\mathrm{~m}, 2 \mathrm{H}) ;{ }^{13} \mathrm{C} \mathrm{NMR}\left(100 \mathrm{MHz}, \mathrm{CDCl}_{3}\right) \delta 168.5,159.1$, 133.9, 129.6, 129.5, 117.7, 113.7, 79.6, 78.5, 71.9, 69.4, 55.3, 36.5, 35.4, 34.7, 33.1; HRMS (EI) found 399.1044 [calcd $\mathrm{C}_{18} \mathrm{H}_{26} \mathrm{BrNO}_{4}(\mathrm{M})^{+}$399.1045].

\section{IAEA of 9,10-anti-Substrates: S9 S11}
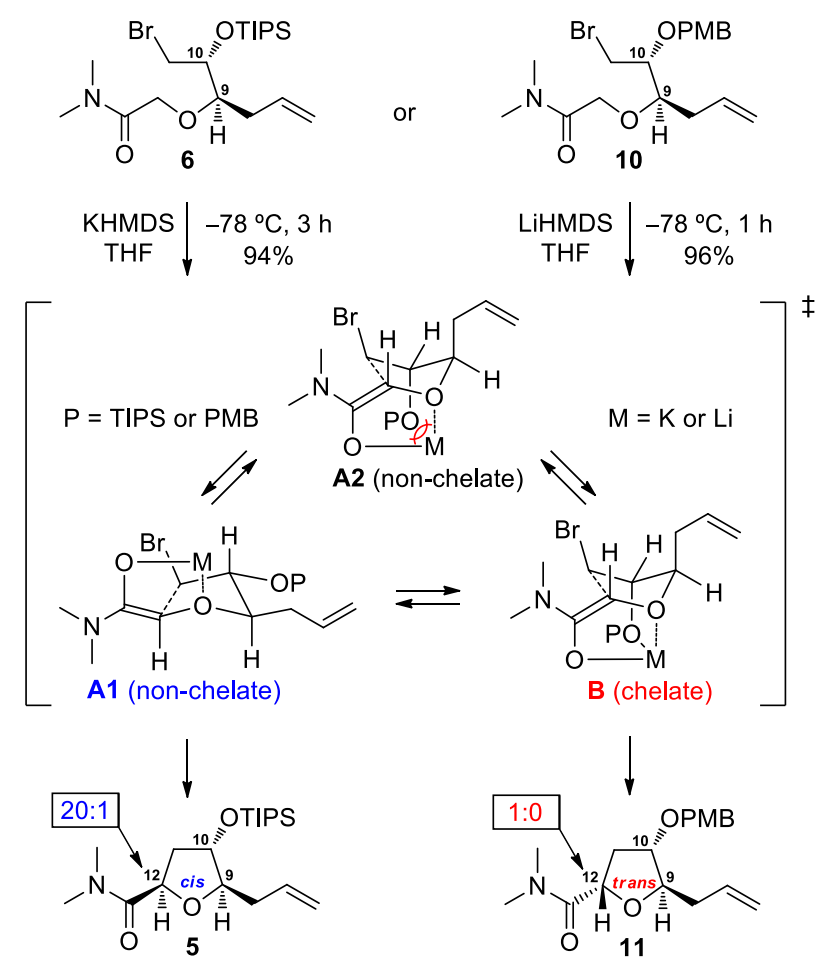


\section{IAEA Reaction of TIPS-protected Bromo Amide 5}

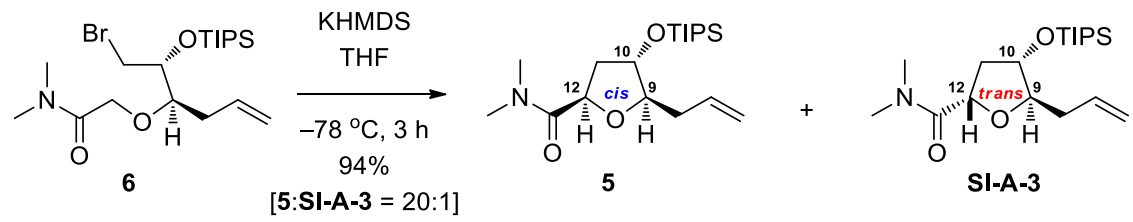

To a cooled $\left(-78^{\circ} \mathrm{C}\right)$ solution of bromo $\alpha$-alkoxy amide $6(75.8 \mathrm{mg}, 0.176 \mathrm{mmol})$ in THF (30 $\mathrm{mL}, 0.006 \mathrm{M})$ were added KHMDS (1.76 mL, 0.5 M solution in THF, $0.88 \mathrm{mmol}$ ) dropwise. The resulting mixture was stirred for $3 \mathrm{~h}$ at the same temperature, quenched with saturated aqueous $\mathrm{NH}_{4} \mathrm{Cl}$, and diluted with EtOAc. The layers were separated, and the aqueous layer was extracted with EtOAc. The combined organic layers were washed with brine, dried over anhydrous $\mathrm{Na}_{2} \mathrm{SO}_{4}$, and concentrated in vасио. The residue was purified by column (silica gel, $n$-hexane/ethyl acetate, 4/1) to afford 9,12-cis-10-TIPSO-THF 5 as a colorless oil (58.0 mg, 94\%) along with trace of amount of 2,5-trans-tetrahydrofuran SI-A-3: [For 9,12-cis-10TIPSO-THF 5] $[\alpha]^{22.5}{ }_{\mathrm{D}}=+14.93\left(c 0.75, \mathrm{CHCl}_{3}\right) ;{ }^{1} \mathrm{H}$ NMR $\left(400 \mathrm{MHz}, \mathrm{CDCl}_{3}\right) \delta 5.81$ (dddd, $J$ $=17.2,10.0,6.8,6.8 \mathrm{~Hz}, 1 \mathrm{H}), 5.07(\mathrm{ddd}, J=16.8,2.8,1.2 \mathrm{~Hz}, 1 \mathrm{H}), 5.04(\mathrm{~d}, J=10.0 \mathrm{~Hz}, 1 \mathrm{H})$, $4.80(\mathrm{dd}, J=8.4,6.8 \mathrm{~Hz}, 1 \mathrm{H}), 4.24(\mathrm{ddd}, J=8.4,5.2,2.8 \mathrm{~Hz}, 1 \mathrm{H}), 3.91$ (ddd, $J=12.8,6.4$, $2.8 \mathrm{~Hz}, 1 \mathrm{H}), 3.13(\mathrm{~s}, 3 \mathrm{H}), 2.96(\mathrm{~s}, 3 \mathrm{H}), 2.49$ (ddd, $J=12.8,8.4,5.2 \mathrm{~Hz}, 1 \mathrm{H}), 2.25$ (dd, $J=$ 6.8, $6.8 \mathrm{~Hz}, 2 \mathrm{H}), 1.96(\mathrm{ddd}, J=9.2,6.8,2.8 \mathrm{~Hz}, 1 \mathrm{H}), 1.06(\mathrm{~s}, 18 \mathrm{H}), 1.05(\mathrm{~s}, 3 \mathrm{H}),{ }^{13} \mathrm{C}$ NMR $(100$ $\left.\mathrm{MHz}, \mathrm{CDCl}_{3}\right) \delta 170.2,134.5,117.0,87.4,75.8,75.5,38.6,37.8,37.2,35.9,18.2,12.3$; IR (neat) 1642, $752 \mathrm{~cm}^{-1}$;HRMS (EI) found 355.2540 [calcd for $\mathrm{C}_{19} \mathrm{H}_{37} \mathrm{NO}_{3} \mathrm{Si}(\mathrm{M})^{+}$355.2543]. [For 9,12trans-10-TIPSO-THF SI-A-3] $[\alpha]^{22.9}{ }_{\mathrm{D}}=+44.26\left(c\right.$ 1.92, $\left.\mathrm{CHCl}_{3}\right) ;{ }^{1} \mathrm{H}$ NMR $\left(400 \mathrm{MHz}, \mathrm{CDCl}_{3}\right)$ $\delta 5.90($ dddd, $J=17.2,10.0,6.8,6.8 \mathrm{~Hz}, 1 \mathrm{H}$ ), 5.16 (dddd, $J=17.2,3.2,1.6,1.6 \mathrm{~Hz}, 1 \mathrm{H}), 5.10$ $(\mathrm{dd}, J=10.0,0.8 \mathrm{~Hz}, 1 \mathrm{H}), 4.70(\mathrm{dd}, J=7.6,7.6 \mathrm{~Hz}, 1 \mathrm{H}), 4.18(\mathrm{ddd}, J=12.8,6.8,6.8 \mathrm{~Hz}, 1$ H), 3.97 (ddd, $J=7.6,6.4,4.8$ Hz, 1 H), 3.11 (s, 3 H), 2.98 (s, 3 H), 2.52-2.44 (m, 1H), 2.442.33(m, 2H), 2.27 (dddd, $J=7.6,7.2,7.2,6.8 \mathrm{~Hz}, 1 \mathrm{H}), 1.09$ (s, $21 \mathrm{H}) ;{ }^{13} \mathrm{C} \mathrm{NMR}(100 \mathrm{MHz}$, $\left.\mathrm{CDCl}_{3}\right) \delta 170.7,134.2,116.8,84.2,75.1,74.5,38.0,36.82,36.76,35.9,18.0,17.9$ 12.2; IR (neat) $1634,752 \mathrm{~cm}^{-1}$; HRMS (EI) found 355.2546 [calcd for $\mathrm{C}_{19} \mathrm{H}_{37} \mathrm{NO}_{3} \mathrm{Si}(\mathrm{M})^{+} 355.2543$ ]. 


\section{IAEA Reaction of PMB-protected Bromo Amide 11}
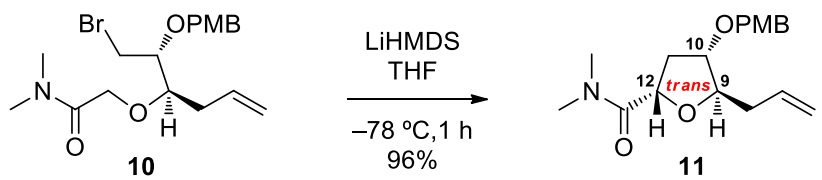

To a cooled $\left(-78{ }^{\circ} \mathrm{C}\right)$ solution of PMB ether $10(124.3 \mathrm{mg}, 0.311 \mathrm{mmol})$ in THF $(60 \mathrm{~mL}, 0.005$ M) was dropwise added LiHMDS $(0.93 \mathrm{~mL}, 1.0 \mathrm{M}$ solution in THF, $0.93 \mathrm{mmol})$. After being stirred for $1 \mathrm{~h}$ at the same temperature, the reaction mixture was quenched with saturated aqueous $\mathrm{NH}_{4} \mathrm{Cl}$, and diluted with EtOAc. The layers were separated, and the aqueous layer was extracted with EtOAc. The combined organic layers were washed with brine, dried over anhydrous $\mathrm{Na}_{2} \mathrm{SO}_{4}$, filtered, and concentrated in vacuo. The residue was purified by column chromatography (silica gel, $n$-hexane/ EtOAc, 1:1) to afford 9,12-trans-10-PMBO-THF 11 as a colorless oil (94.8 mg, 96\%): $[\alpha]^{22}{ }_{\mathrm{D}}=+69.1$ (c 1.04, $\left.\mathrm{CHCl}_{3}\right) ;{ }^{1} \mathrm{H}$ NMR $\left(400 \mathrm{MHz}, \mathrm{CDCl}_{3}\right) \delta 7.24$ (ddd, $J=8.4 \mathrm{~Hz}, 2 \mathrm{H}$ ), 6.85 (ddd, $J=8.0 \mathrm{~Hz}, 2 \mathrm{H}$ ), 5.81 (dddd, $J=16.8,10.0,7.6,7.6 \mathrm{~Hz}, 1 \mathrm{H}$ ), $5.08(\mathrm{~d}, J=16.0 \mathrm{~Hz}, 1 \mathrm{H}), 5.05(\mathrm{~d}, J=9.2 \mathrm{~Hz}, 1 \mathrm{H}), 4.66(\mathrm{dd}, J=7.2,7.2 \mathrm{~Hz}, 1 \mathrm{H}), 4.44(\mathrm{AB}$, $\left.J_{\mathrm{AB}}=11.2 \mathrm{~Hz}, \Delta v_{\mathrm{AB}}=44.0 \mathrm{~Hz}, 2 \mathrm{H}\right), 4.10(\mathrm{ddd}, J=12.0,6.0,6.0 \mathrm{~Hz}, 1 \mathrm{H}), 3.82(\mathrm{dd}, J=12.4$, 6.8, $6.8 \mathrm{~Hz}, 1 \mathrm{H}), 3.78$ (s, $3 \mathrm{H}), 3.05$ (s, $3 \mathrm{H}), 2.93$ (s, $3 \mathrm{H}), 2.48-2.20$ (m, $4 \mathrm{H}) ;{ }^{13} \mathrm{C}$ NMR $(100$ $\left.\mathrm{MHz}, \mathrm{CDCl}_{3}\right) \delta 170.3,158.8,133.8,129.8,129.0,117.0,113.5,82.3,80.9,75.0,71.0,55.1,37.3$, 36.8, 35.9, 34.2; HRMS (CI) found 319.1781[calcd $\mathrm{C}_{18} \mathrm{H}_{25} \mathrm{NO}_{4}(\mathrm{M})^{+} 319.1784$ ]. 


\section{Synthesis of Authentic 9,12-cis-\& 9,12-trans-IAEA Products: S12 S14}
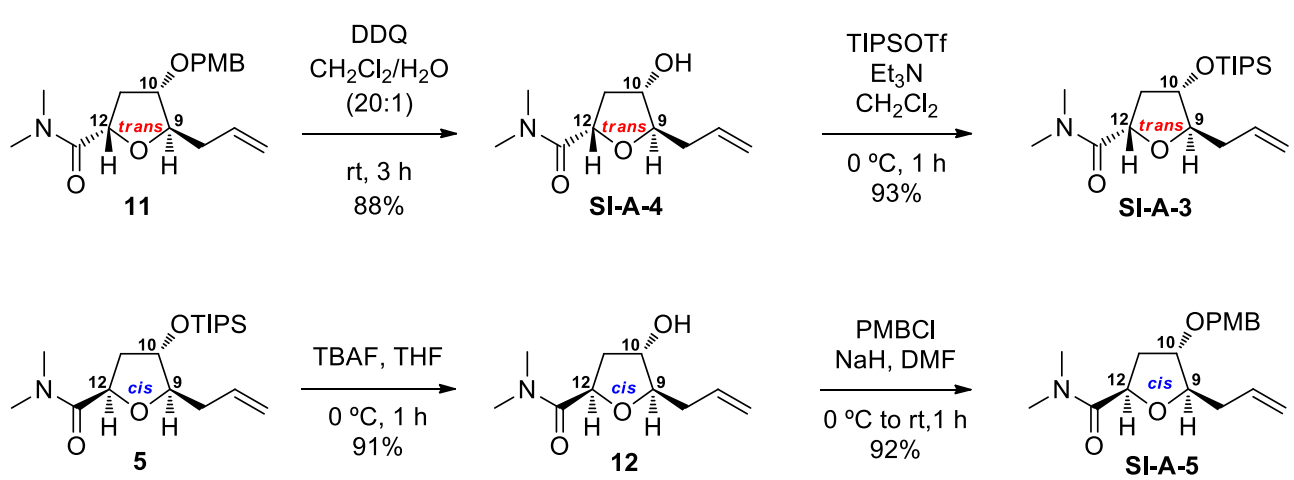

Preparation of 9,12-trans-10-Hydroxy-THF SI-A-4

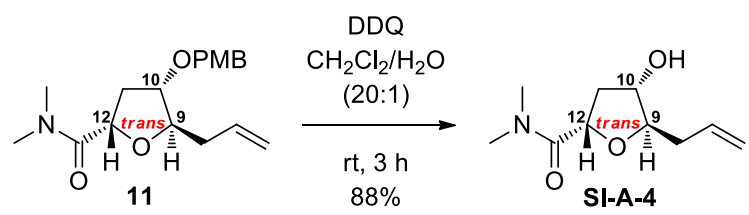

To a solution of 9,12-trans-10-PMBO-THF 11 (134.2 mg, $0.42 \mathrm{mmol})$ in $\mathrm{CH}_{2} \mathrm{Cl}_{2} / \mathrm{H}_{2} \mathrm{O}$ (20:1, $21 \mathrm{~mL}, 0.02 \mathrm{M}$ ) was added 2,3-dichloro-5,6-dicyano-1,4-benzoquinone (DDQ) (190.7 mg, 0.84 $\mathrm{mmol}$ ) at room temperature. After being stirred at the same temperature for $3 \mathrm{~h}$, the resulting mixture was quenched with saturated aqueous $\mathrm{NaHCO}_{3}$, and diluted with $\mathrm{CH}_{2} \mathrm{Cl}_{2}$. The layers were separated, and the aqueous layer was extracted with $\mathrm{CH}_{2} \mathrm{Cl}_{2}$. The combined organic layers were washed with saturated brine, dried over anhydrous $\mathrm{Na}_{2} \mathrm{SO}_{4}$, filtered, and concentrated in vасио. The residue was purified by column chromatography (silica gel, hexanes/ethyl acetate, 1:2 to 1:5) to afford 9,12-trans-10-hydroxy-THF SI-A-4 as a colorless oil $(74.1 \mathrm{mg}, 88 \%):[\alpha]^{22} \mathrm{D}$ $=+75.4\left(c 1.03, \mathrm{CHCl}_{3}\right) ;{ }^{1} \mathrm{H} \mathrm{NMR}\left(400 \mathrm{MHz}, \mathrm{CDCl}_{3}\right) \delta 5.80(\mathrm{dddd}, J=17.2,10.4,6.8,6.8 \mathrm{~Hz}$, $1 \mathrm{H}$ ), 5.14-5.06 (m, $2 \mathrm{H}), 4.97$ (dd, $J=7.6,2.0 \mathrm{~Hz}, 1 \mathrm{H}$ ), 4.95, (s, $1 \mathrm{H}), 4.15$ (ddd, $J=6.8,6.8$, $1.6 \mathrm{~Hz}, 1 \mathrm{H}$ ), 4.07 (ddd, $J=6.0,6.0,6.0 \mathrm{~Hz}, 1 \mathrm{H}$ ), 3.17 (s, $3 \mathrm{H}$ ), 2.99 (s, $3 \mathrm{H}), 2.30-2.17$ (m, $4 \mathrm{H}$ ); ${ }^{13} \mathrm{C}$ NMR $\left(100 \mathrm{MHz}, \mathrm{CDCl}_{3}\right) \delta 172.7,133.7,88.4,75.2$, 74.7, 38.3, 37.5, 36.4, 35.9; HRMS (FAB) found 200.1285 [calcd $\mathrm{C}_{19} \mathrm{H}_{36} \mathrm{O}_{3}(\mathrm{M}+\mathrm{H})^{+}$200.1287]. 


\section{Preparation of 9,12-trans-10-TIPSO-THF SI-A-3}

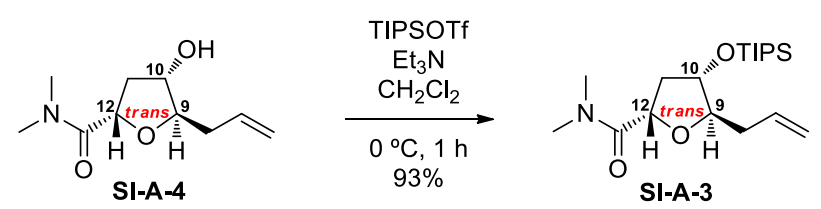

To a cooled $\left(0^{\circ} \mathrm{C}\right)$ solution of 9,12-trans-10-hydroxy-THFSI-A-4 $(74.1 \mathrm{mg}, 0.371 \mathrm{mmol})$ in $\mathrm{CH}_{2} \mathrm{Cl}_{2}(5 \mathrm{~mL}, 0.0371 \mathrm{M})$ were added $\mathrm{Et}_{3} \mathrm{~N}(0.517,3.71 \mathrm{mmol})$ and TIPSOTf $(0.2 \mathrm{~mL}, 0.742$ $\mathrm{mmol})$. The resulting mixture was stirred for $1 \mathrm{~h}$ at the same temperature, quenched with $\mathrm{MeOH}$, and diluted with saturated aqueous $\mathrm{NaHCO}_{3}$ and excess EtOAc. The layers were separated, and the aqueous layer was extracted with EtOAc. The combined organic layers were washed with brine, dried over anhydrous $\mathrm{Na}_{2} \mathrm{SO}_{4}$, and concentrated in vacuo. The residue was purified by column ( $n$-hexane/ethyl acetate $=4 / 1$ ) to afford 9,12-trans-10-TIPSO-THF SI-A-3 as a yellow oil (122.4mg, 93\%).

\section{Preparation of 9,12-cis-10-Hydroxy-THF 12}

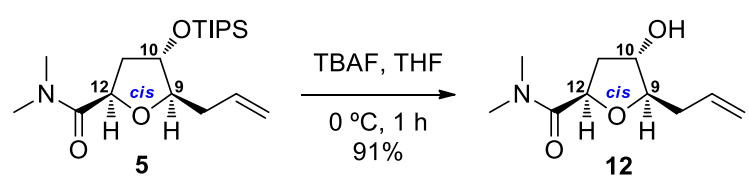

To a solution of 9,12-cis-10-TIPSO-THF 5 (726.1 $\mathrm{mg}, 2.04 \mathrm{mmol})$ in THF (20 mL $0.1 \mathrm{M})$ was added TBAF (2.04 mL, 1.0 M solution in THF, $2.04 \mathrm{mmol})$ dropwise at room temperature. The resulting mixture was stirred at the same temperature for $1 \mathrm{~h}$, quenched with saturated aqueous $\mathrm{NH}_{4} \mathrm{Cl}$, and diluted with $\mathrm{Et}_{2} \mathrm{O}$. The layers were separated, and the aqueous layer was extracted with $\mathrm{Et}_{2} \mathrm{O}$. The combined organic layers were washed with brine, dried over anhydrous $\mathrm{Na}_{2} \mathrm{SO}_{4}$, and concentrated in vacuo. The residue was purified by column chromatography (silica gel, $n$-hexane/ethyl acetate, 10/1 to 5/1) to afford 9,12-cis-10-hydroxyTHF 12 as a colorless oil (371.6 mg, 91\%): $[\alpha]^{23.4}{ }_{\mathrm{D}}=-0.46\left(c 1.61, \mathrm{CHCl}_{3}\right) ;{ }^{1} \mathrm{H}$ NMR (400 $\left.\mathrm{MHz} \mathrm{CDCl}_{3}\right) \delta 5.81(\mathrm{dddd}, J=17.2,10.4,7.2,7.2 \mathrm{~Hz}, 1 \mathrm{H}), 5.11$ (dddd, $J=17.2,3.6,1.6,1.6$ Hz, $1 \mathrm{H}), 5.08$ (dd, $J=10.4,1.2 \mathrm{~Hz}, 1 \mathrm{H}), 4.81$ (dd, $J=6.8,6.8 \mathrm{~Hz}, 1 \mathrm{H}), 4.24$ (ddd, $J=6.0$, 3.2, 3.2 Hz, 1 H), 3.90 (ddd, $J=7.2,7.2,3.2 \mathrm{~Hz}, 1 \mathrm{H}), 3.12$ (s, $3 \mathrm{H}), 2.96$ (s, $3 \mathrm{H}), 2.61$ (ddd, $J$ $=13.6,8.0,5.6 \mathrm{~Hz}, 1 \mathrm{H}), 2.38-2.20(\mathrm{~m}, 2 \mathrm{H}), 2.00(\mathrm{ddd}, J=13.2,6.8,3.2 \mathrm{~Hz}, 1 \mathrm{H}) 1.78(\mathrm{~s}, 1$ 
$\mathrm{H}){ }^{13} \mathrm{C}$ NMR $\left(100 \mathrm{MHz}, \mathrm{CDCl}_{3}\right) \delta 170.2,134.1,116.9,86.3,74.9,74.6,38.2,37.1,36.8,35.8$;

IR (neat) 3384, $1637 \mathrm{~cm}^{-1}$; HRMS (EI) found 199.1209 [calcd for $\mathrm{C}_{10} \mathrm{H}_{17} \mathrm{NO}_{3}(\mathrm{M})^{+} 199.1208$ ].

\section{Preparation of 9,12-cis-10-PMBO-THF SI-A-5}
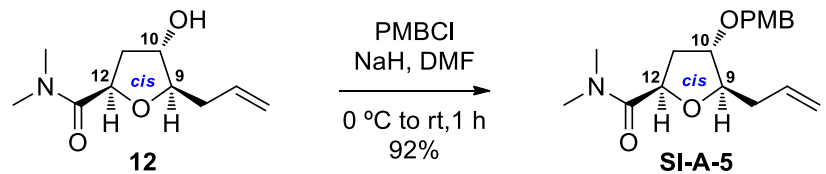

To a cooled $\left(0{ }^{\circ} \mathrm{C}\right)$ solution of 9,12-cis-10-hydroxy-THF $12(64.1 \mathrm{mg}, 0.322 \mathrm{mmol})$ in DMF (3.0 mL, $0.1 \mathrm{M})$ were successively added 4-methoxybenzyl chloride $(0.087 \mathrm{~mL}, 0.644 \mathrm{mmol})$ and $\mathrm{NaH}(51.4 \mathrm{mg}, 60 \%$ dispersion in mineral oil, $1.288 \mathrm{mmol})$. After being stirred for $1 \mathrm{~h}$ at room temperature, the reaction mixture was quenched with saturated aqueous $\mathrm{NH}_{4} \mathrm{Cl}$, and diluted with EtOAc. The layers were separated, and the aqueous layer was extracted with EtOAc. The combined organic layers were washed with saturated brine, dried over anhydrous $\mathrm{Na}_{2} \mathrm{SO}_{4}$, filtered, and concentrated in vacuo. The residue was purified by column chromatography (hexanes/ethyl acetate, 4:1) to afford 9,12-cis-10-PMBO-THF SI-A-5 as a colorless oil (94.2 mg, 92\%): $[\alpha]^{22}=+19.3\left(c 1.00, \mathrm{CHCl}_{3}\right) ;{ }^{1} \mathrm{H}$ NMR $\left(400 \mathrm{MHz}, \mathrm{CDCl}_{3}\right) \delta 7.25$ (ddd, $J=7.6,1.2,1.2$ $\mathrm{Hz}, 2 \mathrm{H}), 6.88$ (d, $J=8.4,2.4,2.4 \mathrm{~Hz}, 2 \mathrm{H}), 5.79$ (dd, $J=17.2,10.4,7.2,7.2 \mathrm{~Hz}, 1 \mathrm{H}), 5.09-5.04$ (m, 2 H), 4.83 (dd, $J=8.8,6.4 \mathrm{~Hz}, 1 \mathrm{H}), 4.44$ (s, 2 H), 4.04 (ddd, $J=9.6,6.4,2.4 \mathrm{~Hz}, 1 \mathrm{H}), 3.92$ (dd, $J=6.0,2.8,2.8 \mathrm{~Hz}, 1 \mathrm{H}), 3.80$ (s, $3 \mathrm{H}), 3.11$ (s, $3 \mathrm{H}), 2.95$ (s, $3 \mathrm{H}), 2.51$ (ddd, $J=14.8,8.4$, $6.4 \mathrm{~Hz}, 1 \mathrm{H}), 2.35-2.19$ (m, $2 \mathrm{H}), 2.08$ (ddd, $J=13.6,6.8,2.8 \mathrm{~Hz}, 1 \mathrm{H}) ;{ }^{13} \mathrm{C}$ NMR $(125 \mathrm{MHz}$, $\left.\mathrm{CDCl}_{3}\right) \delta 169.7,158.9,134.0,129.8,129.1,117.0,113.6,84.2,81.8,75.4,70.9,55.2,38.4,36.9$, 35.7, 34.0. 


\section{Synthesis of Proposed Structure of Bisezakyne A (1a): S15 S22}

\section{Preparation of chloride 4}

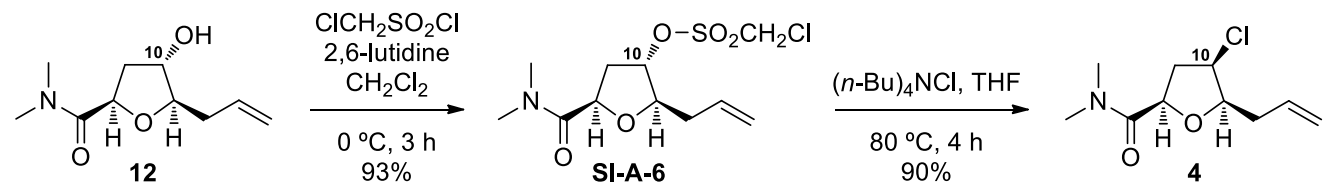

To a cooled $\left(0{ }^{\circ} \mathrm{C}\right)$ solution of alcohol $12(627.4 \mathrm{mg}, 3.15 \mathrm{mmol})$ in $\mathrm{CH}_{2} \mathrm{Cl}_{2}(6 \mathrm{~mL}, 0.5 \mathrm{M})$ were added 2,6-lutidine $(7.33 \mathrm{~mL}, 62.98 \mathrm{mmol})$ and chloromethanesulfonyl chloride $(4.76 \mathrm{~mL}$, $47.23 \mathrm{mmol}$ ) dropwise. The resulting mixture was stirred for $3 \mathrm{~h}$ at the same temperature, quenched with saturated aqueous $\mathrm{NH}_{4} \mathrm{Cl}$, and diluted with $\mathrm{CH}_{2} \mathrm{Cl}_{2}$. The layers were separated, and the aqueous layer was extracted with $\mathrm{CH}_{2} \mathrm{Cl}_{2}$. The combined organic layers were washed with $\mathrm{H}_{2} \mathrm{O}$ and brine, dried over anhydrous $\mathrm{Na}_{2} \mathrm{SO}_{4}$, and concentrated in vacuo. The residue was filtered through a short pad of silica gel ( $n$-hexane/EtOAc, 2/1 to 1/1) to afford the crude chloromethanesulfonate SI-A-6 (1.324 g, 93\%) as a brown oil, which was immediately used for the next step: ${ }^{1} \mathrm{H}$ NMR $\left(400 \mathrm{MHz}, \mathrm{CDCl}_{3}\right) \delta 5.78$ (dddd, $J=17.6,10.8,6.8,6.8 \mathrm{~Hz}, 1 \mathrm{H}$ ), 5.16-5.11 (m, $3 \mathrm{H}), 4.83(\mathrm{dd}, J=9.2,6.0 \mathrm{~Hz}, 1 \mathrm{H}), 4.62(\mathrm{~s}, 2 \mathrm{H}), 4.29$ (ddd, $J=6.4,6.4,1.2 \mathrm{~Hz}$, $1 \mathrm{H}), 3.13(\mathrm{~s}, 3 \mathrm{H}), 2.97(\mathrm{~s}, 3 \mathrm{H}), 2.84$ (ddd, $J=14.8,9.2,6.0 \mathrm{~Hz}, 1 \mathrm{H}), 2.39-2.52(\mathrm{~m}, 3$ H).[ $\mathbf{S}_{\mathbf{N}} \mathbf{2}$ Displacement] To a solution of the crude chloromethanesulfonate SI-A-6 (1.324 g, $4.248 \mathrm{mmol})$ in THF $(20 \mathrm{~mL}, 0.212 \mathrm{M})$ was added $n$-tetrabutylammonium chloride $(5.903 \mathrm{~g}$, $21.24 \mathrm{mmol}$ ) at room temperature. The resulting mixture was stirred at $80^{\circ} \mathrm{C}$ for $4 \mathrm{~h}$, quenched with $\mathrm{H}_{2} \mathrm{O}$ at room temperature, and diluted with EtOAc. The layers were separated, and the aqueous layer was extracted with EtOAc. The layers were separated, and the aqueous layer was extracted with EtOAc. The combined organic layers were washed with brine, dried over anhydrous $\mathrm{Na}_{2} \mathrm{SO}_{4}$, and concentrated in vacuo. The residue was purified by column (silica gel, $n$-hexane/EtOAc, $3 / 1$ to $1 / 1)$ to afford chloride $\mathbf{6}(831.3 \mathrm{mg}, 90 \%)$ as a colorless oil: $[\alpha]^{23.8}{ }_{\mathrm{D}}=-$ 12.97 (c 1.41, $\mathrm{CHCl}_{3}$ ); ${ }^{1} \mathrm{H}$ NMR (400 MHz, $\left.\mathrm{CDCl}_{3}\right) \delta 5.82$ (dddd, $J=16.8,10.0,7.2,7.2 \mathrm{~Hz}, 1$ H), $5.17(\mathrm{~d}, J=17.2 \mathrm{~Hz}, 1 \mathrm{H}), 5.09(\mathrm{ddd}, J=10.0,1.2,1.2 \mathrm{~Hz}, 1 \mathrm{H}), 4.59(\mathrm{dd}, J=8.8,5.6 \mathrm{~Hz}$, $1 \mathrm{H}$ ), 4.41 (ddd, $J=6.4,3.2,3.2 \mathrm{~Hz}, 1 \mathrm{H}$ ), 3.91 (ddd, $J=7.2,7.2,4.0 \mathrm{~Hz}, 1 \mathrm{H}$ ), 3.14 (s, $3 \mathrm{H}$ ), 3.01-2.94 (m, $1 \mathrm{H}), 2.97$ (s, $3 \mathrm{H}), 2.64$ (ddd, $J=15.2,9.2,6.4 \mathrm{~Hz}, 1 \mathrm{H}), 2.59-2.46$ (m, $2 \mathrm{H}$ ); 
${ }^{13} \mathrm{C}$ NMR $\left(100 \mathrm{MHz}, \mathrm{CDCl}_{3}\right) \delta 168.4,133.5,117.4,82.8,75.6,59.9,38.3,37.1,36.1,35.1$; IR (neat) 1649, $1292 \mathrm{~cm}^{-1}$; HRMS (EI) found 217.0870 [calcd for $\mathrm{C}_{10} \mathrm{H}_{16} \mathrm{ClNO}_{2}(\mathrm{M})^{+} 217.0870$ ].

\section{Preparation of Ketone SI-A-7}
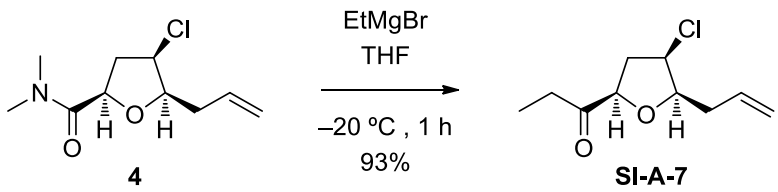

To a cooled $\left(-20^{\circ} \mathrm{C}\right)$ solution of amide $4(311.5 \mathrm{mg}, 1.43 \mathrm{mmol})$ in anhydrous THF (10 $\mathrm{mL}$, $0.14 \mathrm{M})$ was added ethylmagnesium bromide $(0.48 \mathrm{~mL}, 3.0 \mathrm{M}$ solution in ether, $1.44 \mathrm{mmol})$ dropwise. The resulting mixture was stirred at the same temperature for $1 \mathrm{~h}$, quenched with saturated aqueous $\mathrm{NH}_{4} \mathrm{Cl}$, and diluted with $\mathrm{Et}_{2} \mathrm{O}$. The layers were separated, and the aqueous layer was extracted with $\mathrm{Et}_{2} \mathrm{O}$. The combined organic layers were washed with brine, dried over anhydrous $\mathrm{Na}_{2} \mathrm{SO}_{4}$, and concentrated in vacuo. The residue was purified by column chromatography (silica gel, $n$-hexane/EtOAc, 8/1 to 4/1) to afford ketone SI-A-7 (267.7 mg, 93\%) as a colorless oil: $[\alpha]^{20.4}=+28.21\left(\mathrm{c} 0.17, \mathrm{CHCl}_{3}\right) ;{ }^{1} \mathrm{H} \mathrm{NMR}\left(400 \mathrm{MHz}, \mathrm{CDCl}_{3}\right) \delta 5.82$ (ddddd, $J=17.2,10.4,6.8,6.8,0.8 \mathrm{~Hz}, 1 \mathrm{H}), 5.21(\mathrm{dd}, J=17.2,1.2 \mathrm{~Hz}, 1 \mathrm{H}), 5.13(\mathrm{ddd}, J=$ $10.4,1.2,0.8 \mathrm{~Hz}, 1 \mathrm{H}), 4.36(\mathrm{dd}, J=2.8,1.4 \mathrm{~Hz}, 1 \mathrm{H}), 4.35(\mathrm{ddd}, J=10.8,3.2,3.2 \mathrm{~Hz}, 1 \mathrm{H})$, 4.02 (ddd, $J=7.2,6.4,2.8 \mathrm{~Hz}, 1 \mathrm{H}$ ), 2.93 (ddddd, $J=18.8,7.2,7.2,7.2,0.4 \mathrm{~Hz}, 1 \mathrm{H}$ ), 2.702.48(m, $5 \mathrm{H}), 1.05$ (ddd, $J=7.6,6.8,0.8 \mathrm{~Hz}, 3 \mathrm{H}) ;{ }^{13} \mathrm{C} \mathrm{NMR}\left(100 \mathrm{MHz}, \mathrm{CDCl}_{3}\right) \delta 213.1,133.1$, 117.9, 83.0, 81.9, 60.5, 40.8, 35.6, 31.3, 7.2; IR (neat) 1717,1294 $\mathrm{cm}^{-1}$; HRMS (EI) found 202.0769 [calcd for $\mathrm{C}_{10} \mathrm{H}_{15} \mathrm{ClO}_{2}(\mathrm{M})^{+} 202.0761$ ].

\section{Preparation of 13(R)-Alcohol 3a}

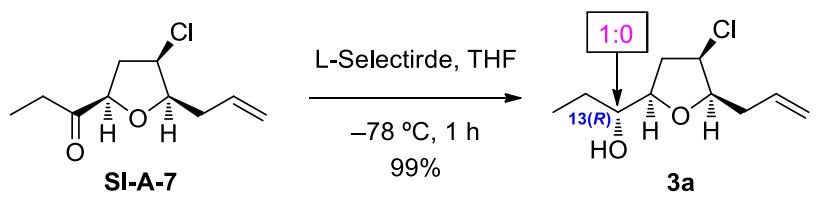

To a cooled $\left(-78{ }^{\circ} \mathrm{C}\right)$ solution of chloro ketone SI-A-7 $(170.8 \mathrm{mg}, 0.84 \mathrm{mmol})$ in THF $(8 \mathrm{~mL}$, $0.1 \mathrm{M}$ )was dropwise added lithium tri-sec-butylborohydride (L-Selectride $\AA$, $0.84 \mathrm{~mL}, 1.0 \mathrm{M}$ solution in THF, $0.84 \mathrm{mmol}$ ). The resulting mixture was stirred at the same temperature for $1 \mathrm{~h}$, quenched with $\mathrm{MeOH}$ and saturated aqueous $\mathrm{NH}_{4} \mathrm{Cl}$. The layers were separated, and the 
aqueous layer was extracted with $\mathrm{Et}_{2} \mathrm{O}$. The combined organic layers were washed with brine, dried over anhydrous $\mathrm{Na}_{2} \mathrm{SO}_{4}$, and concentrated in vacuo. The residue was purified by column chromatography (silica gel, $n$-hexane/ethyl acetate, 100/1 to 20/1) to afford 13(R)-alcohol 3a (172.4 mg, 99\%) as a colorless oil: $[\alpha]^{21.1}{ }_{\mathrm{D}}=-15.45\left(c\right.$ 0.77, $\left.\mathrm{CHCl}_{3}\right)$; ${ }^{1} \mathrm{H}$ NMR $(400 \mathrm{MHz}$, $\left.\mathrm{CDCl}_{3}\right) \delta 5.82(\mathrm{dddd}, J=17.2,10.4,6.8,6.8 \mathrm{~Hz}, 1 \mathrm{H}), 5.18(\mathrm{dd}, J=17.2,1.2 \mathrm{~Hz}, 1 \mathrm{H}), 5.10(\mathrm{dd}$, $J=10.0,0.8 \mathrm{~Hz}, 1 \mathrm{H}$ ), 4.41 (ddd, $J=6.0,3.6,2.0 \mathrm{~Hz}, 1 \mathrm{H}), 3.87-3.79$ (m, $2 \mathrm{H}), 3.54$ (ddd, $J=$ 10.4, 7.6, 7.6 Hz, $1 \mathrm{H}$ ), 2.58 (ddd, $J=14.0,8.8,6.8 \mathrm{~Hz}, 1 \mathrm{H}), 2.55-2.45$ (m, $2 \mathrm{H}), 2.36$ (s, 1H), $2.11(\mathrm{ddd}, J=14.0,6.0,2.0 \mathrm{~Hz}, 1 \mathrm{H}), 1.57-1.40(\mathrm{~m}, 2 \mathrm{H}) 1.01(\mathrm{dd}, J=7.2,7.2 \mathrm{~Hz}, 3 \mathrm{H}) ;{ }^{13} \mathrm{C}$ NMR (100 MHz, $\left.\mathrm{CDCl}_{3}\right) \delta 133.5,117.6,81.6,80.7,74.8,60.9,38.8,35.3,26.3,10.1$; IR (neat) $3452,1292 \mathrm{~cm}^{-1}$; HRMS (EI) found 204.0927 [calcd for $\mathrm{C}_{10} \mathrm{H}_{17} \mathrm{ClO}_{2}(\mathrm{M})^{+} 204.0917$ ].

\section{Determination of Absolute Stereochemistry at $\mathrm{C13}^{2}$}

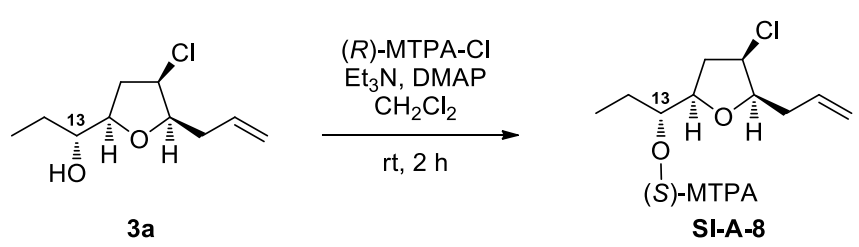

[(S)-MTPA Ester SI-A-8 $]{ }^{1} \mathrm{H}$ NMR $\left(400 \mathrm{MHz}, \mathrm{CDCl}_{3}\right) \delta 7.59(\mathrm{dd}, J=8.0,4.0 \mathrm{~Hz}, 2 \mathrm{H}), 7.40-$ 7.37 (m, $3 \mathrm{H}$ ), 5.75 (dddd, $J=17.2,10.4,6.8,6.8 \mathrm{~Hz}, 1 \mathrm{H}), 5.30$ (ddd, $J=7.6,7.6,3.6 \mathrm{~Hz}, 1 \mathrm{H}$ ), 5.12 (dddd, $J=17.2,2.0,2.0,2.0 \mathrm{~Hz}, 1 \mathrm{H}$ ), 5.05 (dd, $J=10.4,0.8 \mathrm{~Hz}, 1 \mathrm{H}$ ), 4.35 (ddd, $J=6.8$, 3.6, $2.8 \mathrm{~Hz}, 1 \mathrm{H}), 4.01$ (ddd, $J=8.8,7.6,6.0 \mathrm{~Hz}, 1 \mathrm{H}), 3.81$ (ddd, $J=6.8,6.8,4.0 \mathrm{~Hz}, 1 \mathrm{H}$ ), 3.55 (d, $J=1.2 \mathrm{~Hz}, 3 \mathrm{H}), 2.49$ (ddd, $J=14.4,8.4,6.4 \mathrm{~Hz}, 1 \mathrm{H}$ ), 2.43 (dd, $J=7.2,7.2 \mathrm{~Hz}, 2 \mathrm{H}$ ), 2.03 (ddd, $J=14.4,5.6,2.4 \mathrm{~Hz}, 1 \mathrm{H}$ ), 1.77 (ddddd, $J=15.2,7.6,7.6,7.6,3.6 \mathrm{~Hz}, 1 \mathrm{H}), 1.70-1.58$ (m, $1 \mathrm{H}), 0.98(\mathrm{dd}, J=7.2,7.2 \mathrm{~Hz}, 3 \mathrm{H})$.

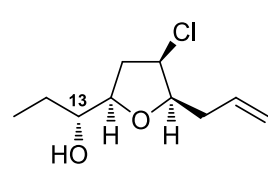

$3 \mathbf{a}$

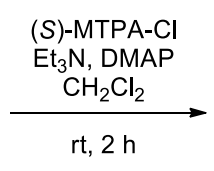

$\mathrm{rt}, 2 \mathrm{~h}$

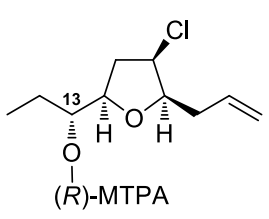

SI-A-9

[(R)-MTPA Ester SI-A-9] ${ }^{1} \mathrm{H}$ NMR $\left(400 \mathrm{MHz}, \mathrm{CDCl}_{3}\right) \delta 7.64(\mathrm{dd}, J=7.6,3.6 \mathrm{~Hz}, 2 \mathrm{H}), 7.39-$ 7.35 (m, $3 \mathrm{H}$ ), 5.79 (dddd, $J=16.8,10.0,6.8,6.8 \mathrm{~Hz}, 1 \mathrm{H}$ ), 5.33 (ddd, $J=8.4,8.4,3.2 \mathrm{~Hz}, 1 \mathrm{H}$ ), 5.15 (dddd, $J=16.8,0.8,0.8,0.8 \mathrm{~Hz}, 1 \mathrm{H}$ ), 5.07 (dd, $J=10.8,1.2 \mathrm{~Hz}, 1 \mathrm{H}$ ), 4.41 (ddd, $J=6.0$, 3.6, $2.0 \mathrm{~Hz}, 1 \mathrm{H}$ ), 4.04 (ddd, $J=8.8,8.8,5.2 \mathrm{~Hz}, 1 \mathrm{H}$ ), 3.89 (ddd, $J=6.4,6.4,3.6 \mathrm{~Hz}, 1 \mathrm{H}$ ), 3.62 
(d, $J=1.2 \mathrm{~Hz}, 3 \mathrm{H}), 2.61(\mathrm{ddd}, J=14.8,8.8,6.4 \mathrm{~Hz}, 1 \mathrm{H}), 2.49(\mathrm{ddd}, J=13.2,6.4,6.4 \mathrm{~Hz}, 2 \mathrm{H})$, 2.10 (ddd, $J=14.8,5.6,2.4 \mathrm{~Hz}, 1 \mathrm{H}), 1.69$ (ddddd, $J=15.2,7.6,7.6,7.6,3.2 \mathrm{~Hz}, 1 \mathrm{H}), 1.58-$ $1.45(\mathrm{~m}, 1 \mathrm{H}), 0.82(\mathrm{dd}, J=7.2,7.2 \mathrm{~Hz}, 3 \mathrm{H})$.

\section{Preparation of 13(S)-Bromide 2a}

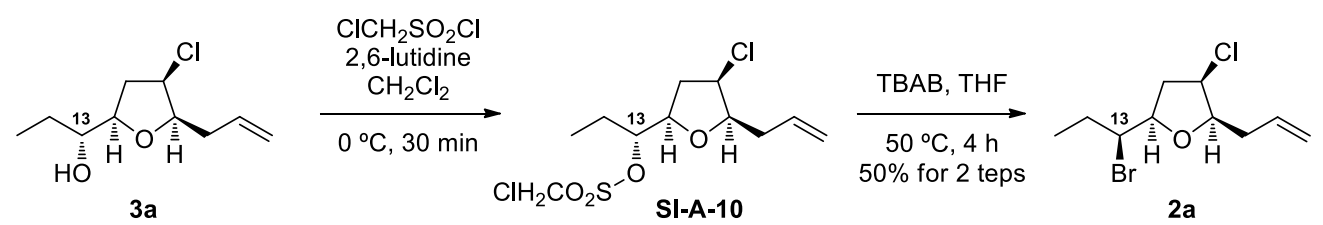

[Sulfonylation] To a cooled $\left(0{ }^{\circ} \mathrm{C}\right)$ solution of $13(R)$-alcohol 3a $(16.5 \mathrm{mg}, 0.081 \mathrm{mmol})$ in $\mathrm{CH}_{2} \mathrm{Cl}_{2}(1 \mathrm{~mL}, 0.081 \mathrm{M})$ were dropwise added 2,6-lutidine $(140.8 \mu \mathrm{L}, 1.215 \mathrm{mmol})$ and chloromethanesulfonyl chloride $(73.2 \mu \mathrm{L}, 0.81 \mathrm{mmol})$. The resulting mixture was stirred for $1 \mathrm{~h}$ at the same temperature, quenched with saturated aqueous $\mathrm{NH}_{4} \mathrm{Cl}$, and diluted with $\mathrm{CH}_{2} \mathrm{Cl}_{2}$. The layers were separated, and the aqueous layer was extracted with $\mathrm{CH}_{2} \mathrm{Cl}_{2}$. The combined organic layers were washed with $\mathrm{H}_{2} \mathrm{O}$ and brine, dried over anhydrous $\mathrm{Na}_{2} \mathrm{SO}_{4}$, and concentrated in vacuo. The residue was filtered through a short pad of silica gel ( $n$-hexane/EtOAc/ $\mathrm{CH}_{2} \mathrm{Cl}_{2}, 4 / 1 / 1$ to $2 / 1 / 1)$ to afford the crude $13(R)$-chloromethanesulfonate SI-A-10 (21.3 $\mathrm{mg}, 90 \%)$ as a brown oil, which was immediately carried onto the next step: ${ }^{1} \mathrm{H}$ NMR $\left(400 \mathrm{MHz}, \mathrm{CDCl}_{3}\right) \delta 5.80$ (dddd, $J=16.8,9.6,6.8,6.8 \mathrm{~Hz}, 1 \mathrm{H}), 5.19$ (dd, $J=17.2,1.6 \mathrm{~Hz}, 1 \mathrm{H}), 5.12$ (dd, $J=10.0,0.8$ $\mathrm{Hz}, 1 \mathrm{H}), 4.87(\mathrm{~d}, J=12.4 \mathrm{~Hz}, 1 \mathrm{H}), 4.80(\mathrm{ddd}, J=8.8,8.8,3.2 \mathrm{~Hz}, 1 \mathrm{H}), 4.69$ (d, $J=12.4 \mathrm{~Hz}$, $1 \mathrm{H}), 4.41$ (ddd, $J=6.0,3.2,2.0 \mathrm{~Hz}, 1 \mathrm{H}), 4.09$ (ddd, $J=8.8,8.8,5.2 \mathrm{~Hz}, 1 \mathrm{H}), 3.96$ (ddd, $J=$ 7.2, 6.0, 3.6 Hz, 1 H), 2.68 (ddd, $J=14.8,8.8,6.0 \mathrm{~Hz}, 1 \mathrm{H}), 2.58-2.45$ (m, $2 \mathrm{H}), 2.07$ (ddd, $J=$ 14.8, 5.6, 2.0 Hz, 1 H), 1.80-1.59 (m, $2 \mathrm{H}), 1.08(\mathrm{dd}, J=7.2,7.2 \mathrm{~Hz}, 3 \mathrm{H})$. [S $\mathbf{S}_{\mathbf{N}} 2$ Displacement] To a solution of the crude $13(R)$-chloromethanesulfonate SI-A-10 $(21.3 \mathrm{mg}, 0.067 \mathrm{mmol})$ in THF (1 mL $0.067 \mathrm{M}$ ) was added tetrabutylammoniumbromide (64.9 mg, $0.201 \mathrm{mmol})$. After being stirred at $70{ }^{\circ} \mathrm{C}$ for $5 \mathrm{~h}$, the reaction mixture was cooled to room temperature, quenched with $\mathrm{H}_{2} \mathrm{O}$, and diluted with $\mathrm{Et}_{2} \mathrm{O}$. The layers were separated and the aqueous layer was extracted with $\mathrm{Et}_{2} \mathrm{O}$. The combined organic layers were washed with brine, dried over anhydrous $\mathrm{Na}_{2} \mathrm{SO}_{4}$, and concentrated in vacuo. The residue was purified by column chromatography (silica gel, hexanes/EtOAc, 40/1 to 10/1) to afford $13(S)$-bromide $2 \mathbf{a}(10.3 \mathrm{mg}, 50 \%$, isolated yield for two 
steps) as a colorless oil; $[\alpha]^{21.1}{ }_{\mathrm{D}}=+19.09\left(\mathrm{c} 0.95, \mathrm{CHCl}_{3}\right) ;{ }^{1} \mathrm{H} \mathrm{NMR}\left(400 \mathrm{MHz}, \mathrm{CDCl}_{3}\right) \delta 5.79$ (dddd, $J=17.2,10.4,7.2,7.2 \mathrm{~Hz}, 1 \mathrm{H}), 5.17$ (dd, $J=17.2,1.2 \mathrm{~Hz}, 1 \mathrm{H}$ ), 5.09 (ddd, $J=3.9,3.9$, $10.1 \mathrm{~Hz}, 1 \mathrm{H}), 4.36(\mathrm{ddd}, J=6.0,3.6,2.0 \mathrm{~Hz}, 1 \mathrm{H}), 4.10-4.02(\mathrm{~m}, 2 \mathrm{H}), 3.91$ (ddd, $J=6.8,6.8$, $3.6 \mathrm{~Hz}, 1 \mathrm{H}), 2.73$ (ddd, $J=10.0,7.6,6.4 \mathrm{~Hz}, 1 \mathrm{H}), 2.55-2.42$ (m, $3 \mathrm{H}), 2.18$ (dddd, $J=15.2$, 7.6, 7.2, 7.2, $2.4 \mathrm{~Hz}, 1 \mathrm{H}$ ), 1.74 (ddddd, $J=15.2,7.6,7.6,7.2,7.2 \mathrm{~Hz}, 1 \mathrm{H}$ ), 1.07 (ddd, $J=7.6$, $7.2 \mathrm{~Hz}, 3 \mathrm{H}){ }^{13} \mathrm{C} \mathrm{NMR}\left(175 \mathrm{MHz}, \mathrm{CDCl}_{3}\right) \delta 133.8,118.1,83.1,80.7,61.5,60.5,42.0,35.8$, 28.4, 11.7.

\section{Preparation of Wittig Salt 13}
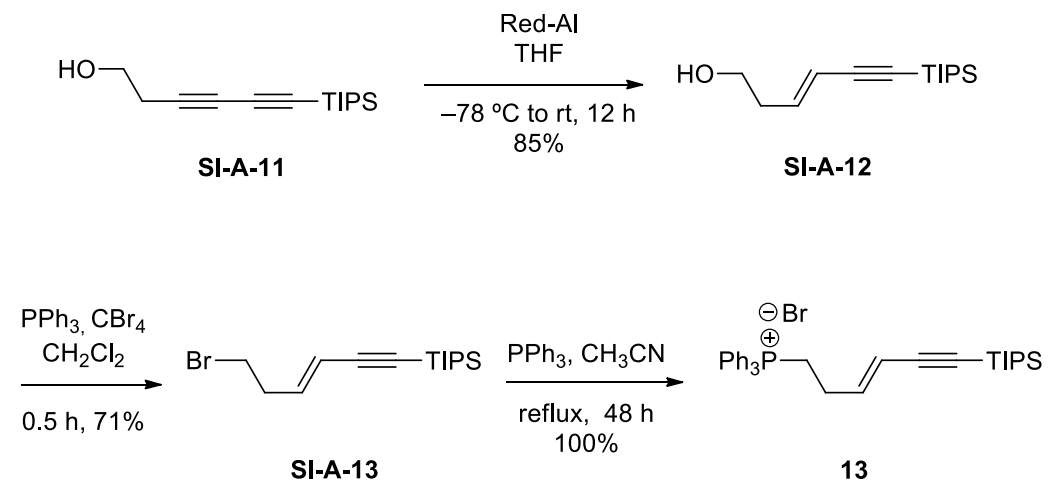

\section{Preparation of $(E)$-Homoallyl Alcohol SI-A-12}

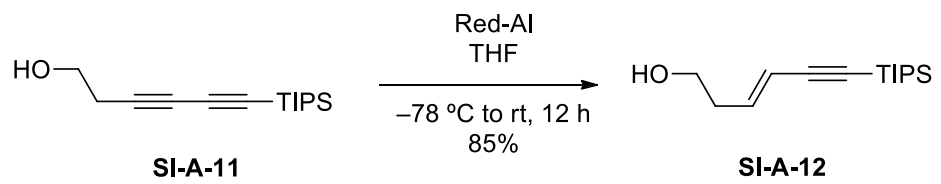

To a solution of known alcohol SI-A-11 (3.84 g, $15.3 \mathrm{mmol})$ in THF (100 ml, $0.15 \mathrm{M})$ was added Red-Al ${ }^{\circledR}(60 \mathrm{wt} \%$ in toluene, $7.49 \mathrm{~mL}, 23.0 \mathrm{mmol})$ at $-78^{\circ} \mathrm{C}$. After being stirred for $12 \mathrm{~h}$ at room temperature, the reaction mixture was cooled to $0{ }^{\circ} \mathrm{C}$, quenched with saturated aqueous $\mathrm{K} / \mathrm{Na}$ tartrate. The resulting mixture was diluted with $\mathrm{Et}_{2} \mathrm{O}$, and stirred vigorously for $2 \mathrm{~h}$. The layers were separated and the aqueous layer was extracted with $\mathrm{Et}_{2} \mathrm{O}$. The combined organic layers were washed with brine, dried over anhydrous $\mathrm{Na}_{2} \mathrm{SO}_{4}$, and concentrated in vacuo. The residue was purified by column chromatography (silica gel; hexane only) to afford alcohol SI-A12 as a coloress oil $(3.29 \mathrm{mg}, 85 \%):{ }^{1} \mathrm{H} \mathrm{NMR}\left(400 \mathrm{MHz}, \mathrm{CDCl}_{3}\right) \delta 6.18(\mathrm{ddd}, J=16.4,7.6,7.6$ Hz, $1 \mathrm{H}), 5.63$ (ddd, $J=15.6,1.2,1.2 \mathrm{~Hz}, 1 \mathrm{H}), 3.68$ (dd, $J=6.4,6.4 \mathrm{~Hz}, 2 \mathrm{H}), 2.37$ (dddd, $J=$ 
6.4, 6.4, 6.4, $1.6 \mathrm{~Hz}, 2 \mathrm{H}), 2.09$ (s, $1 \mathrm{H}), 1.07$ (s, $21 \mathrm{H}) ;{ }^{13} \mathrm{C} \mathrm{NMR}\left(100 \mathrm{MHz}, \mathrm{CDCl}_{3}\right) \delta 141.0$, 112.6, 105.3, 89.6, 61.4, 39.4, 18.7, 11.4.

Preparation of Bromide SI-A-13

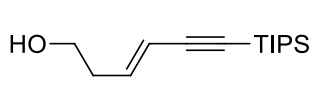

SI-A-12

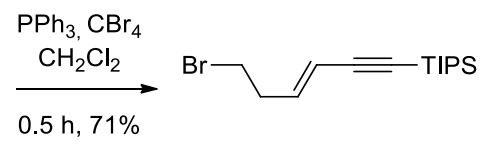

SI-A-13

To a solution of known alcohol SI-A-12 (429.5 mg, $1.701 \mathrm{mmol})$ in $\mathrm{CH}_{2} \mathrm{Cl}_{2}(17 \mathrm{ml}, 0.1 \mathrm{M})$ was added carbon tetrabromide $(564 \mathrm{mg}, 1.701 \mathrm{mmol})$, and triphenylphosphine $(892 \mathrm{mg}, 3.402$ $\mathrm{mmol}$ ) at room temperature. The resulting mixture was stirred for $30 \mathrm{~min}$ at room temperature, and concentrated in vacuo. The residue was purified by column chromatography (silica gel; hexane only) to afford bromide SI-A-13 as a white powder (383 mg, 71\%): ${ }^{1} \mathrm{H}$ NMR (400 MHz, $\left.\mathrm{CDCl}_{3}\right) \delta 6.13(\mathrm{ddd}, J=16.0,7.2,7.2 \mathrm{~Hz}, 1 \mathrm{H}), 5.17$ (ddd, $\left.J=15.6,1.2,1.2 \mathrm{~Hz}, 1 \mathrm{H}\right), 3.39$ (dd, $J=7.2,7.2 \mathrm{~Hz}, 2 \mathrm{H}), 2.67$ (dddd, $J=6.8,6.8,6.8,1.2 \mathrm{~Hz}, 2 \mathrm{H}), 1.08(\mathrm{~s}, 21 \mathrm{H})$.

\section{Preparation of Wittig Salt 13}

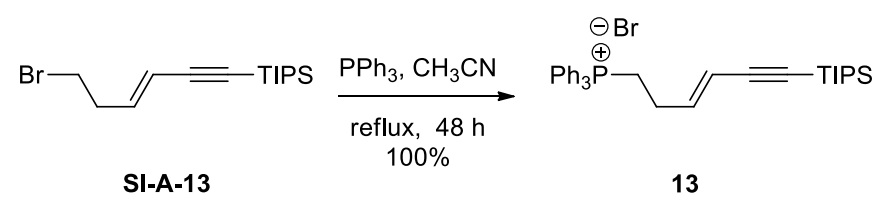

To a solution of bromide SI-A-13 $(292.8 \mathrm{mg}, 0.928 \mathrm{mmol})$ in acetonitrile $(2 \mathrm{~mL}, 0.46 \mathrm{M})$ was added $\mathrm{PPh}_{3}(731.0 \mathrm{mg}, 2.79 \mathrm{mmol})$. The resulting mixture was stirred at $100{ }^{\circ} \mathrm{C}$ for $24 \mathrm{~h}$, cooled, and concentrated in vacuo. The residue was directly purified by column chromatography (silica gel; $\left.\mathrm{CH}_{2} \mathrm{Cl}_{2} / \mathrm{MeOH}, 20 / 1\right)$ to afford Wittig salt 13 as a brown foam (538.0 mg, 100\%): ${ }^{1} \mathrm{H} \mathrm{NMR}$ $\left(400 \mathrm{MHz}, \mathrm{CDCl}_{3}\right) \delta 7.89(\mathrm{dd}, J=12.8,8.4 \mathrm{~Hz}, 6 \mathrm{H}), 7.78(\mathrm{ddd}, J=7.6,7.6,1.2 \mathrm{~Hz}, 3 \mathrm{H}), 7.69$ $(\mathrm{ddd}, J=8.0,8.0,2.8 \mathrm{~Hz}, 6 \mathrm{H}), 6.13(\mathrm{ddd}, J=15.6,6.8,6.8 \mathrm{~Hz}, 1 \mathrm{H}), 5.44(\mathrm{~d}, J=15.6 \mathrm{~Hz}, 1 \mathrm{H})$, 4.12 (ddd, $J=15.2,7.2,7.2 \mathrm{~Hz}, 2 \mathrm{H}), 2.60$ (dddd, $J=14.0,6.8,6.8,6.8 \mathrm{~Hz}, 1 \mathrm{H}), 1.04$ (s, $21 \mathrm{H}$ ). 


\section{Completion of Synthesis of Proposed Structure of Bisezakyne A (1a)}
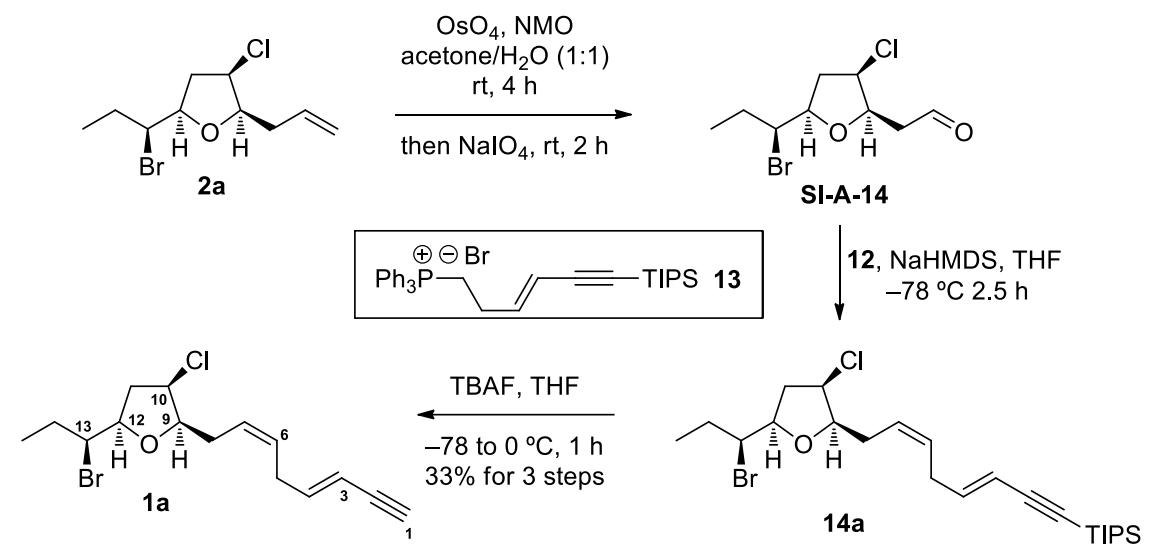

[Lemieux-Johnson Oxidation] To a solution of 13(S)-bromide 2a (10.8 $\mathrm{mg}, 0.040 \mathrm{mmol})$ in acetone $(0.5 \mathrm{~mL}, 0.08 \mathrm{M})$ were dropwise added $\mathrm{OsO}_{4}\left(0.5 \mathrm{wt} \%\right.$ in $\left.\mathrm{H}_{2} \mathrm{O}, 0.25 \mathrm{~mL}, 0.0051 \mathrm{mmol}\right)$ and $\mathrm{NMO}\left(50 \mathrm{wt} \%\right.$ in $\left.\mathrm{H}_{2} \mathrm{O}, 0.25 \mathrm{~mL}, 1.224 \mathrm{mmol}\right)$ at room temperature, and the resulting mixture was stirred for $4 \mathrm{~h}$ at the same temperature. To the mixture was then added $\mathrm{NaIO}_{4}(17.3$ $\mathrm{mg}, 0.080 \mathrm{mmol}$ ), and the resulting mixture was stirred at room temperature for $2 \mathrm{~h}$, diluted with $\mathrm{H}_{2} \mathrm{O}$ and $\mathrm{CH}_{2} \mathrm{Cl}_{2}$. The layers were separated, and the aqueous layer was extracted with $\mathrm{CH}_{2} \mathrm{Cl}_{2}$. The combined organic layers were washed with $\mathrm{H}_{2} \mathrm{O}$ and brine, dried over anhydrous $\mathrm{Na}_{2} \mathrm{SO}_{4}$, and concentrated in vacuo to afford the crude aldehyde SI-A-14 (20.8 mg) as a colorless oil. Without further purification, the crude aldehyde was carried onto the next step: ${ }^{1} \mathrm{H}$ NMR $\left(400 \mathrm{MHz}, \mathrm{CDCl}_{3}\right) \delta 9.79(\mathrm{~s}, 1 \mathrm{H}), 4.55(\mathrm{ddd}, J=6.0,4.0,2.0 \mathrm{~Hz}, 1 \mathrm{H}), 4.38(\mathrm{ddd}, J=6.8$, 6.8, 4.0 Hz, $1 \mathrm{H}), 4.06$ (ddd, $J=9.2,9.2,7.2 \mathrm{~Hz}, 1 \mathrm{H}), 4.01$ (dd, $J=9.2,3.2 \mathrm{~Hz}, 1 \mathrm{H}), 2.95$ (dd, $J=6.4,2.4 \mathrm{~Hz}, 2 \mathrm{H}), 2.78(\mathrm{ddd}, J=14.8,8.0,6.4 \mathrm{~Hz}, 1 \mathrm{H}), 2.45(\mathrm{ddd}, J=14.8,4.8,1.6 \mathrm{~Hz}, 1$ H), 2.14 (ddddd, $J=14.4,7.2,7.2,7.2,2.8 \mathrm{~Hz}, 1 \mathrm{H}), 1.72$ (dddd, $J=14.8,7.6,7.6,7.2 \mathrm{~Hz}, 1 \mathrm{H}$ ), $1.07(\mathrm{dd}, J=7.2,7.2 \mathrm{~Hz}, 3 \mathrm{H})$. [Wittig Reaction] To a cooled $\left(-78^{\circ} \mathrm{C}\right)$ suspension of $(E)$ triphenyl(6-(triisopropylsiyl)hex-3-en-5-yn-1yl)phosphonium bromide 13 (100 mg, $0.173 \mathrm{mmol}$ ) in dry THF ( $1 \mathrm{~mL}, 0.173 \mathrm{M})$ was added dropwise NaHMDS $(0.173 \mathrm{~mL}, 1 \mathrm{M}$ solution in THF, $0.173 \mathrm{mmol})$. To the mixture was added the crude aldehyde SI-A-14 (20.8 $\mathrm{mg}, 0.040 \mathrm{mmol})$ in dry THF $(1 \mathrm{~mL}, 0.040 \mathrm{M})$ at the same temperature. After being stirred for $1 \mathrm{~h}$, the reaction mixture was quenched with saturated aqueous $\mathrm{NH}_{4} \mathrm{Cl}$, and diluted with $\mathrm{Et}_{2} \mathrm{O}$. The layers were separated, and the aqueous layer was extracted with $\mathrm{Et}_{2} \mathrm{O}$. The combined organic layers were 
washed with brine, dried over anhydrous $\mathrm{Na}_{2} \mathrm{SO}_{4}$, and concentrated in vacuo. The residue was purified by column chromatography(silica gel, $n$-hexane/ethyl acetate, 40/1)to afford TIPS-(E)enyne 14a as a colorless oil (15.1 mg, $72 \%$ yield for two steps): ${ }^{1} \mathrm{H}$ NMR (400 $\mathrm{MHz}, \mathrm{CDCl}_{3}$ ) $\delta 6.18$ (ddd, $J=15.6,6.0,6.0 \mathrm{~Hz}, 1 \mathrm{H}$ ), 5.55 (ddd, $J=12.8,2.0,2.0 \mathrm{~Hz}, 1 \mathrm{H}$ ), 5.91 (ddd, $J=6.0$, $3.2,3.2 \mathrm{~Hz}, 2 \mathrm{H}$ ), 4.34 (ddd, $J=5.2,3.2,1.6 \mathrm{~Hz}, 1 \mathrm{H}$ ), $4.10-4.02(\mathrm{~m}, 2 \mathrm{H}), 3.87$ (ddd, $J=6.0,6.0$, $3.2 \mathrm{~Hz}, 1 \mathrm{H}$ ), 2.90 (ddd, $J=6.0,6.0,1.6 \mathrm{~Hz}, 2 \mathrm{H}), 2.72$ (ddd, $J=14.8,6.4,4.0 \mathrm{~Hz}, 1 \mathrm{H}$ ), 2.552.40 (m, 3H), 2.18 (ddddd, $J=14.8,7.6,7.6,7.6,2.8 \mathrm{~Hz}, 1 \mathrm{H}$ ), 1.74 (ddddd, $J=14.8,7.6,7.6$, 7.6, 7.6, Hz, $1 \mathrm{H}), 1.07$ (dd, $J=7.6,7.6 \mathrm{~Hz}, 3 \mathrm{H}), 1.07$ (s, 21H). [Deprotection] To a cooled (0 $\left.{ }^{\circ} \mathrm{C}\right)$ solution of TIPS- $(E)$-enyne 14a $(15.1 \mathrm{mg}, 0.031 \mathrm{mmol})$ in THF $(2 \mathrm{~mL}, 0.020 \mathrm{M})$ was added dropwise TBAF $(0.1 \mathrm{~mL}, 1.0 \mathrm{M}$ solution in THF, $0.1 \mathrm{mmol})$. The resulting mixture wasstirred for 30 min, quenched with saturated aqueous $\mathrm{NH}_{4} \mathrm{Cl}$, and diluted with $\mathrm{Et}_{2} \mathrm{O}$.The layers were separated and the aqueous layer was extracted with $\mathrm{Et}_{2} \mathrm{O}$. The combined organic layers were dried over anhydrous $\mathrm{Na}_{2} \mathrm{SO}_{4}$ concentrated in vacuo. The residue was purified by column chromatography (silica gel, $n$-hexane/ethylacetate, 100/1 to 40/1) to afford proposed structure of bisezakyne A (1a) as a colorless oil (7.0 mg, 33\% isolated yield for three steps): $[\alpha]_{\mathrm{D}}^{19.4}=$ $+19.11\left(c 0.75, \mathrm{CHCl}_{3}\right) ;{ }^{1} \mathrm{H}$ NMR $\left(400 \mathrm{MHz}, \mathrm{CDCl}_{3}\right) \delta 6.23$ (ddd, $J=16.4,6.8,6.8 \mathrm{~Hz}, 1 \mathrm{H}$ ), $5.53-5.45(\mathrm{~m}, 3 \mathrm{H}), 4.34$ (ddd, $J=6.0,3.2,1.6 \mathrm{~Hz}, 1 \mathrm{H}), 4.07$ (ddd, $J=9.2,9.2,4.4 \mathrm{~Hz}, 1 \mathrm{H}$ ), 4.04 (ddd, $J=9.6,9.6,2.8 \mathrm{~Hz}, 1 \mathrm{H}$ ), 3.87 (ddd, $J=6.8,6.8,3.6 \mathrm{~Hz}, 1 \mathrm{H}$ ), 2.91 (dd, $J=6.0$, $2.0 \mathrm{~Hz}, 2 \mathrm{H}), 2.80(\mathrm{~d}, J=2.4 \mathrm{~Hz}, 1 \mathrm{H}), 2.72(\mathrm{ddd}, J=14.4,8.4,6.0 \mathrm{~Hz}, 1 \mathrm{H}), 2.57-2.40(\mathrm{~m}, 3 \mathrm{H})$, 2.18 (ddddd, $J=14.8,2.8,7.6 \mathrm{~Hz}, 1 \mathrm{H}$ ), 1.74 (ddddd, $J=14.8,8.4,7.6 \mathrm{~Hz}, 1 \mathrm{H}$ ), 1.07 (dd, $J=$ 7.2, 7.2Hz, $3 \mathrm{H}){ }^{13} \mathrm{C}$ NMR $\left(175 \mathrm{MHz}, \mathrm{CDCl}_{3}\right) \delta 144.0,128.1,126.4,109.2,83.0,82.3,80.5$, 76.3, 61.2, 60.4, 41.9, 30.9, 29.5, 28.3, 11.5; IR (neat) 3292, $1285 \mathrm{~cm}^{-1}$; HRMS (EI) found 330.0389 [calcd for $\mathrm{C}_{15} \mathrm{H}_{20} \mathrm{BrClO}(\mathrm{M})^{+} 330.0386$ ]. 


\section{Synthesis of Revised Structure of Bisezakyne A (1b): S23 S27}

\section{Preparation of 13(S)-Alcohol 3b}
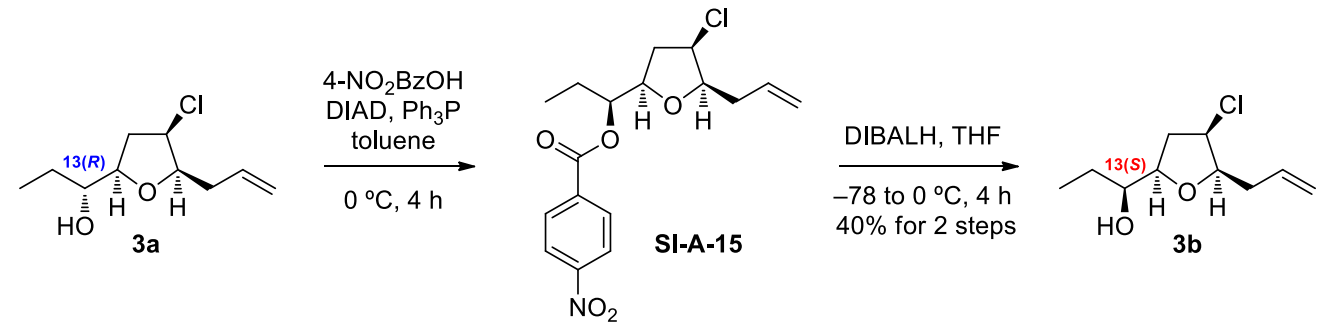

[Mitsunobu Reaction] To a cooled $\left(0{ }^{\circ} \mathrm{C}\right)$ solution of $13(R)$-alcohol 3a $(30.7 \mathrm{mg}, 0.15 \mathrm{mmol})$ in dry THF (3 mL, $0.05 \mathrm{M})$ were sequentially added triphenylphosphine (354 mg, $1.35 \mathrm{mmol})$, diisopropyl azodicarboxylate $(267.6 \mu \mathrm{L}, 1.35 \mathrm{mmol})$ and $p$-nitrobenzoic acid $(225.5 \mathrm{mg}, 1.35$ mmol). The resulting mixture wasstirred at $0{ }^{\circ} \mathrm{C}$ for $3 \mathrm{~h}$, and the reaction mixture was concentrated in vacuo. The residue was directly purified by column chromatography (silica gel, $n$-hexane/ethyl acetate, 20/1 to 8/1) to afford the 13(S)-p-nitrobenzoate SI-A-15 as a colorless oil (24.6 mg, 48\%): ${ }^{1} \mathrm{H}$ NMR (400 MHz, $\left.\mathrm{CDCl}_{3}\right) \delta 8.30-8.24$ (m, $4 \mathrm{H}$ ), 5.78 (dddd, $J=17.2$, $10.0,6.8,6.8 \mathrm{~Hz}, 1 \mathrm{H}), 5.37$ (ddd, $J=8.4,4.8,3.6 \mathrm{~Hz}, 1 \mathrm{H}), 5.13(\mathrm{dd}, J=16.8,1.2 \mathrm{~Hz}, 1 \mathrm{H})$, $5.07(\mathrm{~d}, J=10.0 \mathrm{~Hz}, 1 \mathrm{H}), 4.41(\mathrm{ddd}, J=6.0,3.6,2.0 \mathrm{~Hz}, 1 \mathrm{H}), 4.11$ (ddd, $J=10.8,5.2,5.2 \mathrm{~Hz}$, $1 \mathrm{H}), 3.83$ (ddd, $J=6.8,6.8,3.6 \mathrm{~Hz}, 1 \mathrm{H}$ ), 2.64 (dddd, $J=17.2,10.0,6.8,6.8 \mathrm{~Hz}, 1 \mathrm{H}), 2.53-$ $2.40(\mathrm{~m}, 2 \mathrm{H}), 2.32(\mathrm{ddd}, J=14.8,6.0,2.0 \mathrm{~Hz}, 1 \mathrm{H}), 1.91-1.71(\mathrm{~m}, 2 \mathrm{H}), 0.99$ (dd, J = 8.0, 8.0 Hz, 3 H). [DIBAL-H Reduction] To a cooled $\left(-78^{\circ} \mathrm{C}\right)$ solution of $13(S)$-p-nitrobenzoate SI-A$15(24.6 \mathrm{mg}, 0.070 \mathrm{mmol})$ in anhydrous THF $(1 \mathrm{~mL}, 0.07 \mathrm{M})$ was added dropwise DIBAL-H $(0.12 \mathrm{~mL}, 25 \%$ in toluene, $0.21 \mathrm{mmol})$. The resulting mixture was stirred for $1 \mathrm{~h}$ at the same temperature, quenched with $\mathrm{MeOH}$ and saturated aqueous $\mathrm{NH}_{4} \mathrm{Cl}$ and poured into $\mathrm{Et}_{2} \mathrm{O}$. The layers were separated and the aqueous layer was extracted twice with $\mathrm{Et}_{2} \mathrm{O}$. The combined organic layers were dried over anhydrous $\mathrm{Na}_{2} \mathrm{SO}_{4}$ concentrated in vacuo. The residue was purified by column chromatography (silica gel, $n$-hexane/ethylacetate, $5 / 1$ to $3 / 1$ ) to afford 13(S)-alcohol 3b as a colorless oil $(11.8 \mathrm{mg}, 83 \%)$ : $[\alpha]^{22.0}{ }_{\mathrm{D}}=-10.44\left(c 1.55, \mathrm{CHCl}_{3}\right) ;{ }^{1} \mathrm{H} \mathrm{NMR}$ $\left(400 \mathrm{MHz}, \mathrm{CDCl}_{3}\right) \delta 5.82(\mathrm{dddd}, J=16.8,9.6,6.8,6.8 \mathrm{~Hz}, 1 \mathrm{H}), 5.18(\mathrm{dd}, J=16.8,1.2 \mathrm{~Hz}, 1$ H), $5.11(\mathrm{~d}, J=10.4 \mathrm{~Hz}, 1 \mathrm{H}), 4.42(\mathrm{ddd}, J=6.4,3.6,2.8 \mathrm{~Hz}, 1 \mathrm{H}), 3.89$ (ddd, $J=9.2,6.0,3.2$ Hz, 1 H), 3.84-3.77 (m, 2 H), 2.57-2.42 (m, 3 H), 2.33 (ddd, $J=14.0,6.0,1.2$ Hz, 1 H), 2.23 (s, 
$1 \mathrm{H}), 1.52-1.36(\mathrm{~m}, 2 \mathrm{H}), 0.99(\mathrm{dd}, J=7.2,7.2 \mathrm{~Hz}, 3 \mathrm{H}),{ }^{13} \mathrm{C} \mathrm{NMR}\left(100 \mathrm{MHz}, \mathrm{CDCl}_{3}\right) \delta 133.6$, 117.6, 81.6, 80.7, 72.4, 61.1, 35.9, 35.3, 25.9, 10.3; IR (neat) 3443, $1291 \mathrm{~cm}^{-1}$; HRMS (EI) found 204.0923 [calcd for $\mathrm{C}_{10} \mathrm{H}_{17} \mathrm{ClO}_{2}(\mathrm{M})^{+} 204.0917$ ].

\section{Preparation of $13(R)$-Bromide $2 b$}

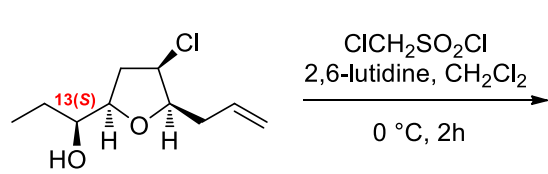

$3 \mathbf{b}$

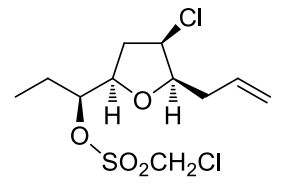

SI-A-16

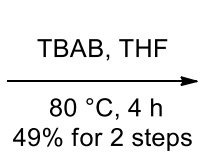

$49 \%$ for 2 steps

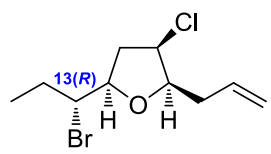

$2 \mathbf{b}$

[Sulfonylation] To a cooled $\left(0{ }^{\circ} \mathrm{C}\right)$ solution of $13(S)$-alcohol $3 \mathbf{b}(51.8 \mathrm{mg}, 0.253 \mathrm{mmol})$ in $\mathrm{CH}_{2} \mathrm{Cl}_{2}(1 \mathrm{~mL}, 0.253 \mathrm{M})$ were dropwise added 2,6-lutidine $(0.442 \mathrm{~mL}, 3.795 \mathrm{mmol})$ and chloromethanesulfonyl chloride $(0.255 \mathrm{~mL}, 2.530 \mathrm{mmol})$. The resulting mixture was stirred for $1 \mathrm{~h}$ at the same temperature, quenched with saturated aqueous $\mathrm{NH}_{4} \mathrm{Cl}$, and diluted with $\mathrm{CH}_{2} \mathrm{Cl}_{2}$. The layers were separated, and the aqueous layer was extracted with $\mathrm{CH}_{2} \mathrm{Cl}_{2}$. The combined organic layers were washed with $\mathrm{H}_{2} \mathrm{O}$ and saturated brine, dried over anhydrous $\mathrm{Na}_{2} \mathrm{SO}_{4}$, and concentrated in vасио. The residue was filtered through a short pad of silica gel ( $n$ hexanes/EtOAc/ $\mathrm{CH}_{2} \mathrm{Cl}_{2}, 4 / 1 / 1$ to $\left.2 / 1 / 1\right)$ to afford the crude $13(R)$-chloromethanesulfonate SI-A$16(76.5 \mathrm{mg}, 95 \%)$ as a brown oil, which was carried onto the next step: ${ }^{1} \mathrm{H}$ NMR (400 MHz, $\left.\mathrm{CDCl}_{3}\right) \delta 5.80(\mathrm{dddd}, J=17.2,10.4,6.8,6.8 \mathrm{~Hz}, 1 \mathrm{H}), 5.18(\mathrm{dd}, J=17.2,1.2 \mathrm{~Hz}, 1 \mathrm{H}), 5.12(\mathrm{dd}$, $J=10.4,1.2 \mathrm{~Hz}, 1 \mathrm{H}), 4.92(\mathrm{ddd}, J=6.8,5.6,3.6 \mathrm{~Hz}, 1 \mathrm{H}), 4.75$ (s, $2 \mathrm{H}), 4.45$ (ddd, $J=6.8,3.6$, $2.4 \mathrm{~Hz}, 1 \mathrm{H}), 3.99$ (ddd, $J=8.4,6.4,3.6 \mathrm{~Hz}, 1 \mathrm{H}), 3.83$ (ddd, $J=10.8,6.8,4.0 \mathrm{~Hz}, 1 \mathrm{H}), 2.63$ (ddd, $J=14.8,8.4,7.6 \mathrm{~Hz}, 1 \mathrm{H}), 2.53-2.40$ (m, $2 \mathrm{H}), 2.36$ (ddd, $J=14.4,6.8,2.0 \mathrm{~Hz}, 1 \mathrm{H}$ ), 1.80-1.72 (m, $2 \mathrm{H}) 1.07(\mathrm{dd}, J=7.6,7.2 \mathrm{~Hz}, 3 \mathrm{H})$. [ $\mathbf{S}_{\mathbf{N}} 2$ Displacement] To a solution of the 13(S)-chloromethanesulfonate SI-A-16 $(76.5 \mathrm{mg}, 0.241 \mathrm{mmol})$ in THF (10 mL $0.024 \mathrm{M})$ was added TBAB (466.4 mg, $0.723 \mathrm{mmol}$ ), and the resulting mixture was stirred at $70{ }^{\circ} \mathrm{C}$ for $5 \mathrm{~h}$. The reaction mixture was cooled to room temperature, quenched with $\mathrm{H}_{2} \mathrm{O}$, and diluted with $\mathrm{Et}_{2} \mathrm{O}$. The layers were separated and the aqueous layer was extracted with $\mathrm{Et}_{2} \mathrm{O}$. The combined organic layers were dried over anhydrous $\mathrm{Na}_{2} \mathrm{SO}_{4}$ concentrated in vacuo. The residue was purified by column chromatography (silica gel, hexanes/EtOAc, 20/1 to 10/1) to afford 13(R)bromide $2 \mathbf{b}$ as a colorless oil $(33.8 \mathrm{mg}, 50 \%$ for 2 steps $):[\alpha]^{22.2}{ }_{\mathrm{D}}=-2.16\left(c 0.99, \mathrm{CHCl}_{3}\right) ;{ }^{1} \mathrm{H}$ NMR (400 MHz, $\mathrm{CDCl}_{3}$ ) $\delta 5.82$ (dddd, $J=17.2,10.4,6.8,6.8 \mathrm{~Hz}, 1 \mathrm{H}$ ), 5.18 (dddd, $J=16.8$, 
3.2, 1.6, 1.6 Hz, $1 \mathrm{H}), 5.10(\mathrm{ddd}, J=10.4,2.0,0.8 \mathrm{~Hz}, 1 \mathrm{H}), 4.43$ (ddd, $J=6.4,4.0,2.4 \mathrm{~Hz}, 1$ H), 4.16 (ddd, $J=8.4,6.4,6.4 \mathrm{~Hz}, 1 \mathrm{H}), 4.05$ (ddd, $J=10.0,6.4,3.2 \mathrm{~Hz}, 1 \mathrm{H}$ ), 3.87 (ddd, $J=$ 7.2, 7.2, 4.0 Hz, $1 \mathrm{H}), 2.65$ (ddd, $J=15.2,8.4,7.2 \mathrm{~Hz}, 1 \mathrm{H}), 2.60-2.46(\mathrm{~m}, 2 \mathrm{H}), 2.24(\mathrm{ddd}, J=$ 15.2, 6.8, $2.4 \mathrm{~Hz}, 1 \mathrm{H}$ ), 2.03 (ddddd, $J=14.8,7.6,7.6,7.2,3.2 \mathrm{~Hz}, 1 \mathrm{H}$ ), 1.75 (dddd, $J=14.8$, 10.4, 7.2, 7.2 Hz, $1 \mathrm{H}), 1.10(\mathrm{dd}, J=7.6,7.2 \mathrm{~Hz}, 3 \mathrm{H}) ;{ }^{13} \mathrm{C} \mathrm{NMR}\left(175 \mathrm{MHz}, \mathrm{CDCl}_{3}\right) \delta 133.8$, $118.1,82.4,80.8,60.5,59.2,39.6,35.5,27.0,12.4$; IR (neat) $1290 \mathrm{~cm}^{-1}$.

\section{Completion of Synthesis of Revised Structure of Bisezakyne A (1b)}

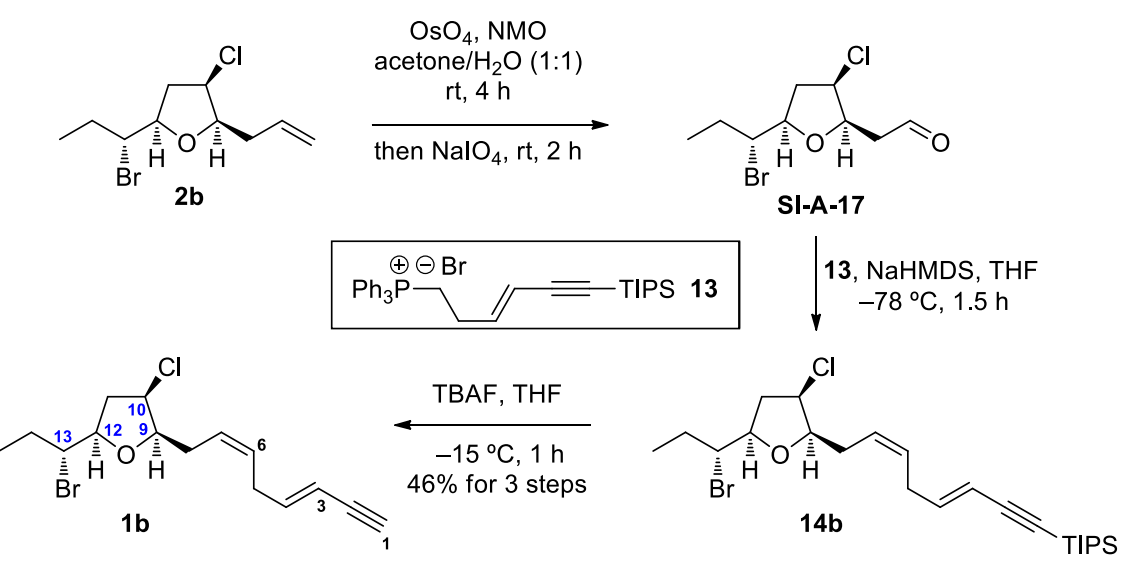

[Lemieux-Johnson Oxidation] To a solution of 13(R)-bromide $2 \mathbf{b}$ (23.9 $\mathrm{mg}, 0.089 \mathrm{mmol})$ in acetone $(0.5 \mathrm{~mL}, 0.178 \mathrm{M})$ were sequentially added $\mathrm{OsO}_{4}\left(0.5 \mathrm{wt} \%\right.$ in $\mathrm{H}_{2} \mathrm{O}, 0.25 \mathrm{~mL}, 0.0051$ mmol) and NMO (50 wt\% in $\left.\mathrm{H}_{2} \mathrm{O}, 0.25 \mathrm{~mL}, 1.224 \mathrm{mmol}\right)$ at room temperature, and the resulting mixture was stirred for $4 \mathrm{~h}$ at the same temperature. To the resulting mixture was added $\mathrm{NaIO}_{4}(17.3 \mathrm{mg}, 0.080 \mathrm{mmol})$ and the resulting mixture was stirred for $2 \mathrm{~h}$. The reaction mixture was diluted with $\mathrm{H}_{2} \mathrm{O}$ and $\mathrm{CH}_{2} \mathrm{Cl}_{2}$. The layers were separated, and the aqueous layer was extracted with $\mathrm{CH}_{2} \mathrm{Cl}_{2}$. The combined organic layers were washed successively with $\mathrm{H}_{2} \mathrm{O}$ and brine, dried over anhydrous $\mathrm{Na}_{2} \mathrm{SO}_{4}$, and concentrated in vacuo to afford the crude aldehyde SI-A-17 (32.1 mg) as a colorless oil. Without further purification, the crude aldehyde SI-A-17 was carried onto the next step: ${ }^{1} \mathrm{H}$ NMR $\left(400 \mathrm{MHz}, \mathrm{CDCl}_{3}\right) \delta 9.80(\mathrm{~s}, 1 \mathrm{H}), 4.63$ (ddd, $J=6.4,4.0,2.4 \mathrm{~Hz}, 1 \mathrm{H}), 4.35$ (ddd, $J=6.4,6.4,4.4 \mathrm{~Hz}, 1 \mathrm{H}), 4.14$ (ddd, $J=6.4,6.4,2.4 \mathrm{~Hz}, 1$ H), $4.02(\mathrm{ddd}, J=6.4,6.4,3.2 \mathrm{~Hz}, 1 \mathrm{H}), 3.01(\mathrm{dd}, J=7.2,2.4 \mathrm{~Hz}, 2 \mathrm{H}), 2.71(\mathrm{ddd}, J=15.2,7.6$, $7.6 \mathrm{~Hz}, 1 \mathrm{H}), 2.25$ (ddd, $J=15.2,7.2,2.8 \mathrm{~Hz}, 1 \mathrm{H}), 1.99$ (ddddd, $J=14.4,7.2,7.2,7.2,3.2 \mathrm{~Hz}$, 
$1 \mathrm{H}), 1.82-1.70(\mathrm{~m}, 1 \mathrm{H}) 1.10(\mathrm{dd}, J=7.2,7.2 \mathrm{~Hz}, 3 \mathrm{H})$. [Wittig Reaction] A cooled $\left(-78{ }^{\circ} \mathrm{C}\right)$ suspension of (E)-triphenyl(6-(triisopropylsiyl)hex-3-en-5-yn-1yl)phosphonium bromide $\mathbf{1 3}$ $(180.0 \mathrm{mg}, 0.361 \mathrm{mmol})$ in dry THF $(2 \mathrm{~mL}, 0.18 \mathrm{M})$ was added dropwise NaHMDS $(0.36 \mathrm{~mL}$, 1.0 M solution in THF, $0.360 \mathrm{mmol}$ ). To the mixture was added the crude aldehyde SI-A-17 (20.8 $\mathrm{mg}, 0.040 \mathrm{mmol})$ in dry THF $(1 \mathrm{~mL}, 0.040 \mathrm{M})$ at the same temperature. After being stirred for $1 \mathrm{~h}$ at the same temperature, the reaction mixture was quenched with saturated aqueous $\mathrm{NH}_{4} \mathrm{Cl}$, and diluted with $\mathrm{Et}_{2} \mathrm{O}$.The layers were separated, and the aqueous layer was extracted with $\mathrm{Et}_{2} \mathrm{O}$. The combined organic layers were washed with brine, dried over anhydrous $\mathrm{Na}_{2} \mathrm{SO}_{4}$, and concentrated in vacuo. The residue was purified by column chromatography(silica gel, $n$-hexane/ethyl acetate, 40/1) to afford TIPS-(E)-enyne $\mathbf{1 4 b}$ as a colorless oil (28.9 mg, $66 \%$ for 2 steps): ${ }^{1} \mathrm{H}$ NMR $\left(400 \mathrm{MHz}, \mathrm{CDCl}_{3}\right) \delta 6.18$ (ddd, $J=16.0,6.8$, $6.8 \mathrm{~Hz}, 1 \mathrm{H}), 5.55$ (ddd, $J=15.6,2.0,2.0 \mathrm{~Hz}, 1 \mathrm{H}), 5.55-5.44(\mathrm{~m}, 2 \mathrm{H}), 4.42$ (ddd, $J=6.0,3.6$, $2.0 \mathrm{~Hz}, 1 \mathrm{H}$ ), 4.15 (ddd, $J=8.8,6.4,6.4 \mathrm{~Hz}, 1 \mathrm{H}$ ), 4.05 (ddd, $J=9.6,6.0,2.8 \mathrm{~Hz}, 1 \mathrm{H}$ ), 3.83 (ddd, $J=7.2,7.2,4.4 \mathrm{~Hz}, 1 \mathrm{H}), 2.91(\mathrm{dd}, J=6.0,6.0 \mathrm{~Hz}, 2 \mathrm{H}), 2.56(\mathrm{ddd}, J=15.2,8.8,7.6 \mathrm{~Hz}$, $1 \mathrm{H}$ ), 2.60-2.43 (m, 2 H), 2.40 (ddd, $J=14.8,6.4,2.0 \mathrm{~Hz}, 1 \mathrm{H}$ ), 2.02 (ddddd, $J=14.8,7.2,7.2$, 7.2, $2.8 \mathrm{~Hz}, 1 \mathrm{H}$ ), 1.75 (ddddd, $J=14.8,10.0,7.2,7.2,7.2 \mathrm{~Hz}, 1 \mathrm{H}), 1.10$ (dd, $J=7.2,7.2 \mathrm{~Hz}, 3$ $\mathrm{H}), 1.074(\mathrm{~s}, 3 \mathrm{H}), 1.069$ (s, $18 \mathrm{H})$. [Deprotection] To a cooled $\left(0{ }^{\circ} \mathrm{C}\right)$ solution of TIPS- $(E)$ enyne $14 \mathbf{b}(26.3 \mathrm{mg}, 0.057 \mathrm{mmol})$ in THF $(2 \mathrm{~mL}, 0.029 \mathrm{M})$ was added dropwise TBAF $(0.1 \mathrm{~mL}$, $1.0 \mathrm{M}$ solution in THF, $0.1 \mathrm{mmol}$ ). After being stirred for $30 \mathrm{~min}$, the reaction was quenched with saturated aqueous $\mathrm{NH}_{4} \mathrm{Cl}$ and diluted with $\mathrm{Et}_{2} \mathrm{O}$. The layers were separated and the aqueous layer was extracted with $\mathrm{Et}_{2} \mathrm{O}$. The combined organic layers were dried over anhydrous $\mathrm{Na}_{2} \mathrm{SO}_{4}$ concentrated in vacuo. The residue was purified by column chromatography (silica gel, $n$-hexane/ethylacetate, $5 / 1$ to 3/1) to afford revised structure of bisezakyne A (1b) as a colorless oil (13.1 mg, 70\%): $[\alpha]^{21.9}{ }_{\mathrm{D}}=-5.56\left(c 0.14, \mathrm{CHCl}_{3}\right) ;{ }^{1} \mathrm{H} \mathrm{NMR}\left(400 \mathrm{MHz}, \mathrm{CDCl}_{3}\right) \delta 6.23(\mathrm{ddd}$, $J=16.0,6.0,6.0 \mathrm{~Hz}, 1 \mathrm{H}), 5.55-5.48$ (m, $2 \mathrm{H}$ ), 5.48 (dddd, $J=16.0,2.0,2.0,2.0 \mathrm{~Hz}, 1 \mathrm{H}$ ), 4.42 (ddd, $J=6.8,3.6,2.0 \mathrm{~Hz}, 1 \mathrm{H}$ ), 4.15 (ddd, $J=8.4,6.4,6.4 \mathrm{~Hz}, 1 \mathrm{H}$ ), 4.05 (ddd, $J=10.0,6.0$, $2.8 \mathrm{~Hz}, 1 \mathrm{H}), 3.83$ (ddd, $J=6.8,6.8,3.6 \mathrm{~Hz}, 1 \mathrm{H}), 2.92(\mathrm{ddd}, J=6.0,6.0,1.2 \mathrm{~Hz}, 2 \mathrm{H}), 2.81$ (d, $J=1.6 \mathrm{~Hz}, 1 \mathrm{H}), 2.65(\mathrm{ddd}, J=14.8,8.4,6.8 \mathrm{~Hz}, 1 \mathrm{H}), 2.57$ (ddd, $J=12.8,6.0,6.0 \mathrm{~Hz}, 1 \mathrm{H}$ ), 2.47 (ddd, $J=13.6,6.8,6.8 \mathrm{~Hz}, 1 \mathrm{H}), 2.24$ (ddd, $J=15.2,6.4,2.4 \mathrm{~Hz}, 1 \mathrm{H}), 2.01$ (ddddd, $J=$ 
14.8, 7.2, 7.2, 7.2, $2.8 \mathrm{~Hz}, 1 \mathrm{H}), 1.75$ (ddddd, $J=14.4,10.0,6.8,6.8,6.8 \mathrm{~Hz}, 1 \mathrm{H}), 1.10$ (dd, $J=$ 7.2, 7.2 Hz, $3 \mathrm{H}) ;{ }^{13} \mathrm{C} \mathrm{NMR}\left(175 \mathrm{MHz}, \mathrm{CDCl}_{3}\right) \delta 144.0,128.2,126.4,109.1,82.3,82.2,80.6$, 76.2, 60.4, 58.9, 39.5, 30.9, 29.2, 27.0, 12.2; IR (neat) $3294 \mathrm{~cm}^{-1}$; HRMS (EI) found 330.0387 [calcd for $\left.\mathrm{C}_{15} \mathrm{H}_{20} \mathrm{BrClO}(\mathrm{M})^{+} 330.0386\right]$.

\section{References}

1. (a) Gao, Y.; Hanson, R. M.; Klunder, J. M.; Ko, S. Y.; Masamune, H.; Sharpless, K. B. J. Am. Chem. Soc. 1987, 109, 5765. (b) Crimmins, M. T.; Powell, M. T. J. Am. Chem. Soc. 2003, 125, 7592. (c) Kim, B.; Sohn, T. I.; Kim, S.; Kim, D.; Lee, J. Heterocycles 2011, 82, 1113.

2. Ohtani, I.; Kusumi, T.; Kashman, Y.; Kakisawa, H. J. Am. Chem. Soc. 1991, 113, 4092. 


\section{Comparative ${ }^{1} \mathrm{H}$ NMR Data for Bisezakyne A}

Synthetic 13(S)-Bisezakyne A

$$
\text { (erythro) }
$$

Synthetic 13(S) (erythro, Proposed)

number

\begin{tabular}{|c|c|}
\hline 1 & $2.80(d, J=2.4)$ \\
\hline \multicolumn{2}{|l|}{2} \\
\hline 3 & $5.53-5.45(\mathrm{~m})$ \\
\hline 4 & $6.23(\mathrm{ddd}, \mathrm{J}=16.4,6.8,6.8)$ \\
\hline 5 & $2.91(d d, J=6.0,2.0)$ \\
\hline 6 & $5.53-5.45(\mathrm{~m})$ \\
\hline 7 & $5.53-5.45(\mathrm{~m})$ \\
\hline \multirow[t]{2}{*}{8} & $2.51(\mathrm{~m})$ \\
\hline & $2.47(\mathrm{~m})$ \\
\hline 9 & 3.87 (ddd, $J=6.8,6.8,3.6$ ) \\
\hline 10 & $4.34(\mathrm{ddd}, \mathrm{J}=6.0,3.2,1.6)$ \\
\hline \multirow[t]{2}{*}{11} & 2.72 (ddd, J= 14.4, 8.4, 6.0) \\
\hline & $2.43(\mathrm{~m})$ \\
\hline 12 & 4.07 (ddd, J= 9.2, 9.2, 4.4) \\
\hline 13 & 4.04 (ddd, J= 9.6, 9.6, 2.8) \\
\hline 14 & 2.18 (ddddd, J= 14.8, 7.6, 7.6, 7.6 2.8) \\
\hline & 1.74 (ddddd, $J=14.8,8.4,7.6,7.6,7.6$ ) \\
\hline
\end{tabular}

15
1.07 (dd, J= 7.2, 7.2)

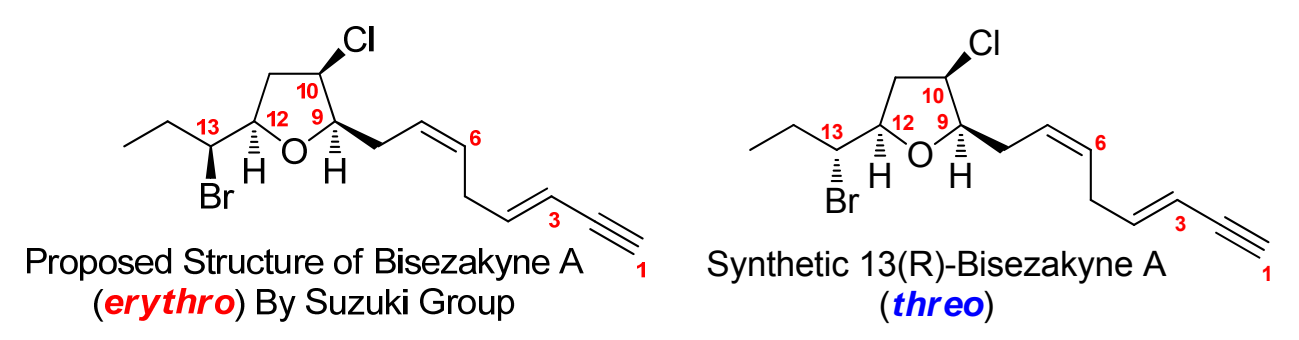

Synthetic 13(R) (threo, Revised)

$2.89(d, J=1.5)$

$2.81(d, J=1.6)$

5.49 (br dd, J=16.1, 6.4, 6.4)

6.24 (ddd, J= 16.1, 6.4, 6.4)

$2.92(\mathrm{~m})$

$5.51(\mathrm{~m})$

$5.51(\mathrm{~m})$

2.56 (ddd, J= 13.7, 6.4, 6.4; $\mathrm{Ha}$ )

2.47 (ddd, J= 13.7, 6.8, 6.8; $\mathrm{Hb}$ )

3.83 (ddd, $J=6.8,6.4,3.6$ )

4.42 (ddd, $J=6.8,3.6,2.4$ )

2.65 (ddd, J= 15.1, 8.3, 6.8; Ha)

2.24 (ddd, J= 15.1, 6.4, 2.4; $\mathrm{Hb}$ )

4.15 (ddd, $J=8.3,6.4,6.4$ )

4.05 (ddd, J= 9.9, 6.4, 2.9)

2.01 (ddddd, $\mathrm{J}=14.6,7.3,7.3,7.3,2.9$ )

1.74 (ddddd, $\mathrm{J}=14.6,9.9,7.3,7.3,7.3$ )

1.10 (dd, J= 7.3, 7.3)
5.48 (dddd, $J=16.0,2.0,2.0,2.0$ )

6.23 (ddd, $J=16.0,6.0,6.0$ )

2.92 (ddd, $J=6.0,6.0,1.2$ )

5.55-5.48 (m)

5.55-5.48 (m)

2.57 (ddd, , J = 12.8, 6.0, 6.0)

2.47 (ddd, , $\mathrm{J}=13.6,6.8,6.8$ )

3.83 (ddd, $\mathrm{J}=6.8,6.8,3.6$ )

4.42 (ddd, J = 6.8, 3.6, 2.0)

2.65 (ddd, J = 14.8, 8.4, 6.8)

2.24 (ddd, $J=15.2,6.4,2.4$ )

4.15 (ddd, $J=8.4,6.4,6.4$ )

4.05 (ddd, $\mathrm{J}=10.0,6.0,2.8$ )

2.01 (ddddd, $\mathrm{J}=14.8,7.2,7.2,7.2,2.8$ )

1.75 (ddddd, $\mathrm{J}=14.4,10.0,6.8,6.8,6.8$ )

1.10 (dd, $\mathrm{J}=7.2,7.2$ ) 


\section{Comparative ${ }^{13} \mathrm{C}$ NMR Data for Bisezakyne A}

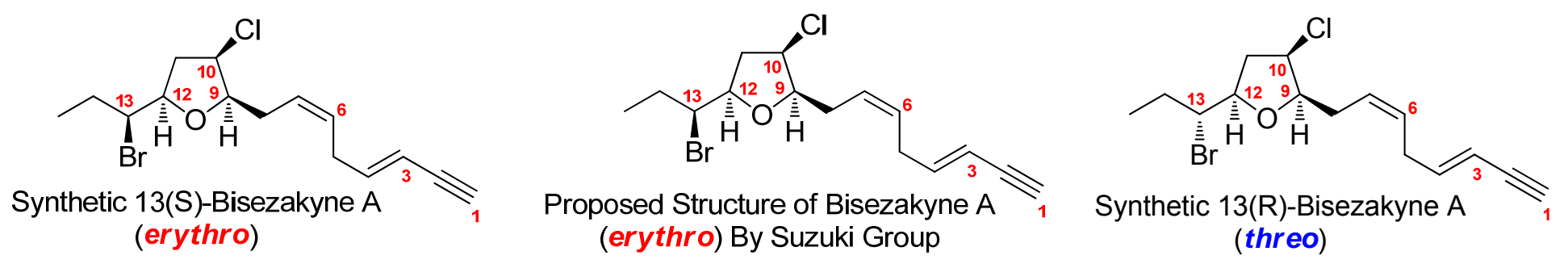

\begin{tabular}{|c|c|c|c|c|c|}
\hline $\begin{array}{l}\text { Carbon } \\
\text { number }\end{array}$ & $\begin{array}{l}\text { Synthetic 13(S) } \\
\text { (Proposed) }\end{array}$ & $\begin{array}{c}\Delta \\
\text { Syn.(erythro)-Nat. }\end{array}$ & Natural & $\begin{array}{c}\Delta \\
\text { Syn.(threo)-Nat. }\end{array}$ & $\begin{array}{c}\text { Synthetic 13(R) } \\
\text { (Revised) }\end{array}$ \\
\hline 1 & 76.3 & 0.0 & 76.3 & -0.1 & 76.2 \\
\hline 2 & 83.0 & 0.6 & 82.4 & -0.1 & 82.3 \\
\hline 3 & 109.2 & 0.1 & 109.1 & 0.0 & 109.1 \\
\hline 4 & 144.0 & 0.0 & 144.0 & 0.0 & 144.0 \\
\hline 5 & 30.9 & 0.0 & 30.9 & 0.0 & 30.9 \\
\hline 6 & 128.1 & -0.1 & 128.2 & 0.0 & 128.2 \\
\hline 7 & 126.4 & 0.0 & 126.4 & 0.0 & 126.4 \\
\hline 8 & 29.5 & 0.3 & 29.2 & 0.0 & 29.2 \\
\hline 9 & 82.3 & 0.1 & 82.2 & 0.0 & 82.2 \\
\hline 10 & 61.2 & 0.8 & 60.4 & 0.0 & 60.4 \\
\hline 11 & 41.9 & 2.4 & 39.5 & 0.0 & 39.5 \\
\hline 12 & 80.5 & -0.1 & 80.6 & 0.0 & 80.6 \\
\hline 13 & 60.4 & 1.4 & 59.0 & -0.1 & 58.9 \\
\hline 14 & 28.3 & 1.3 & 27.0 & 0.0 & 27.0 \\
\hline 15 & 11.5 & -0.7 & 12.2 & 0.0 & 12.2 \\
\hline
\end{tabular}




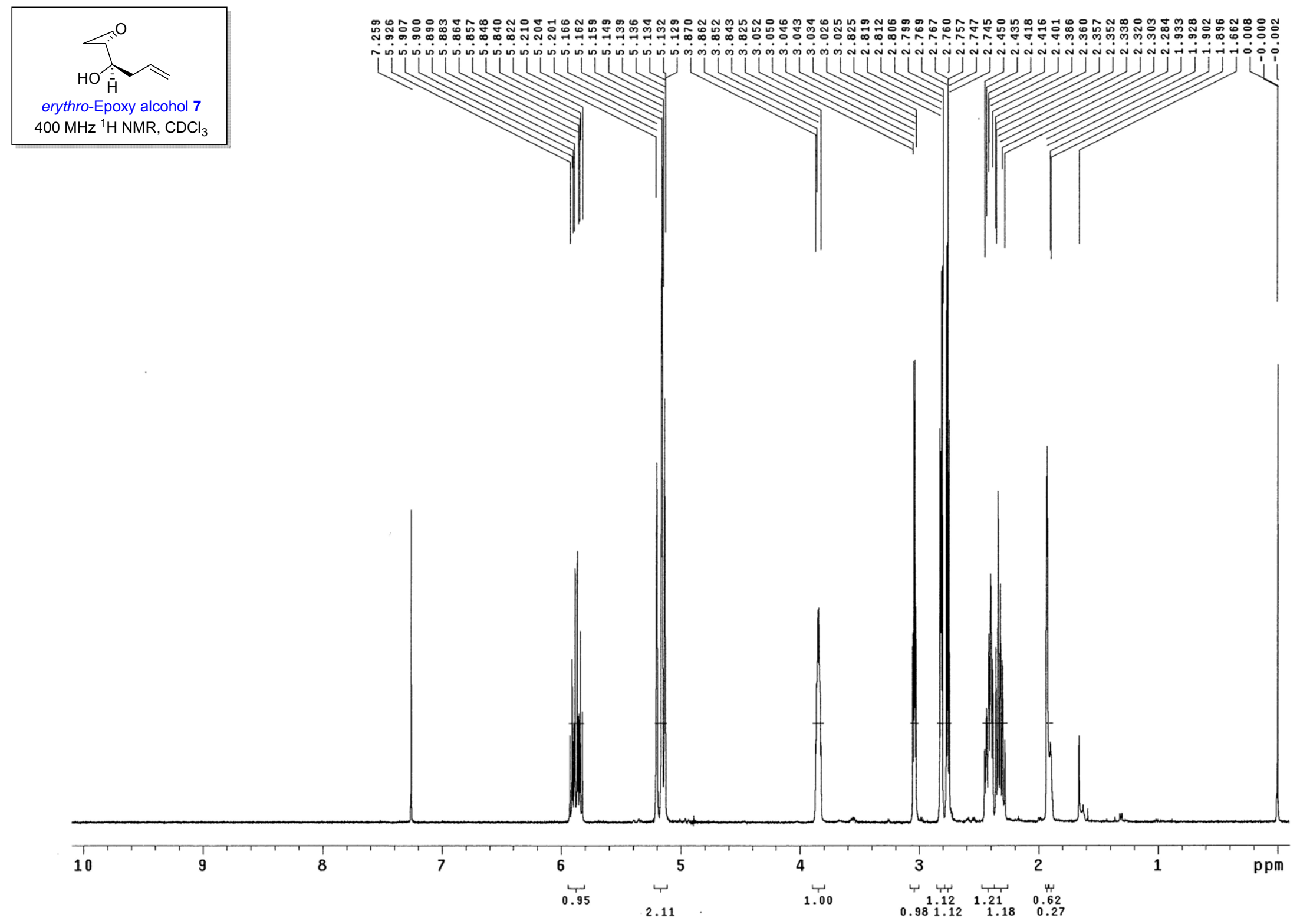




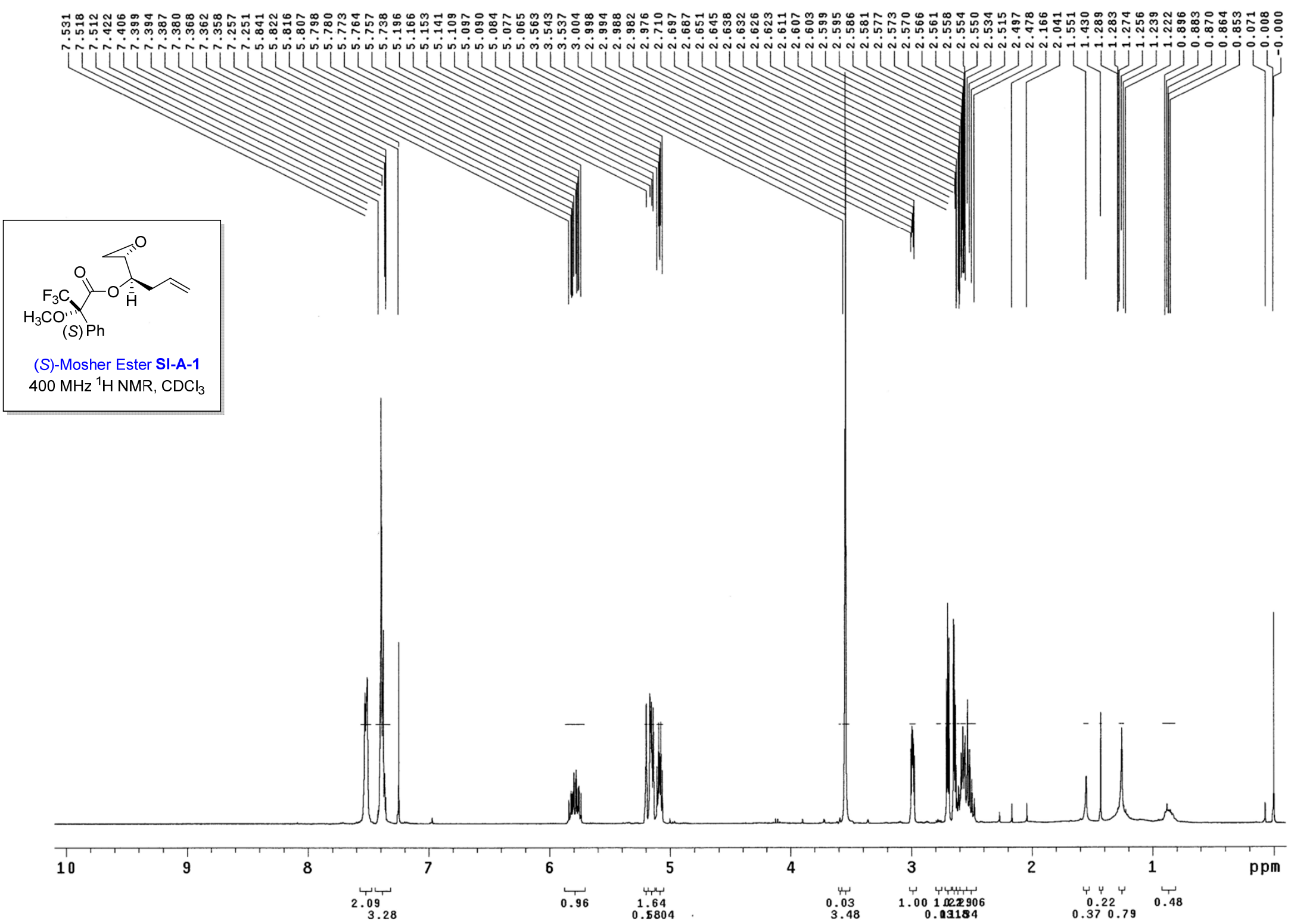




$$
\begin{aligned}
& (R)-\mathrm{Mosher} \text { Ester } \mathrm{SI}-\mathrm{A}-2 \\
& 400 \mathrm{MHz}{ }^{1} \mathrm{H} N M R, \mathrm{CDCl}_{3}
\end{aligned}
$$
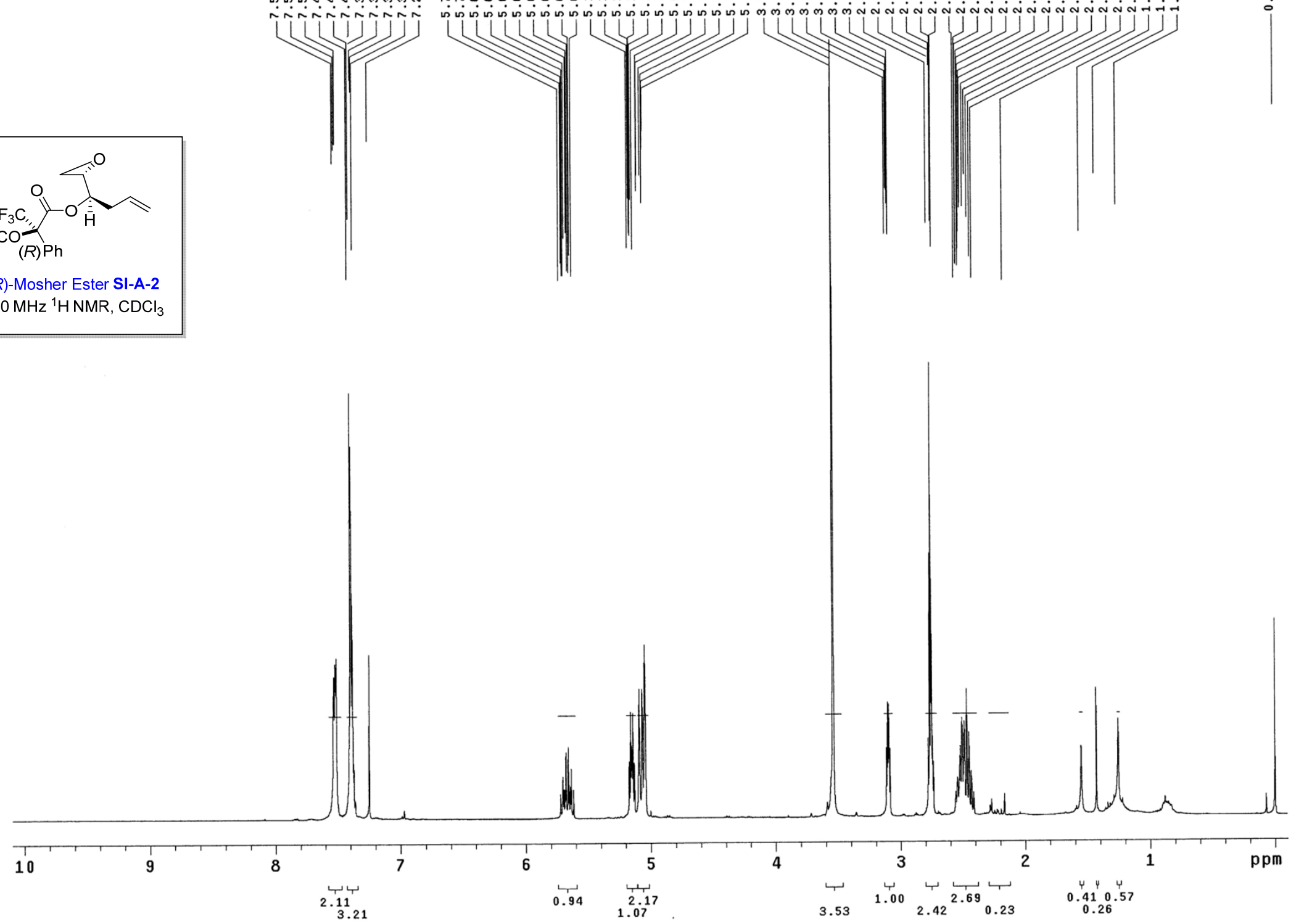


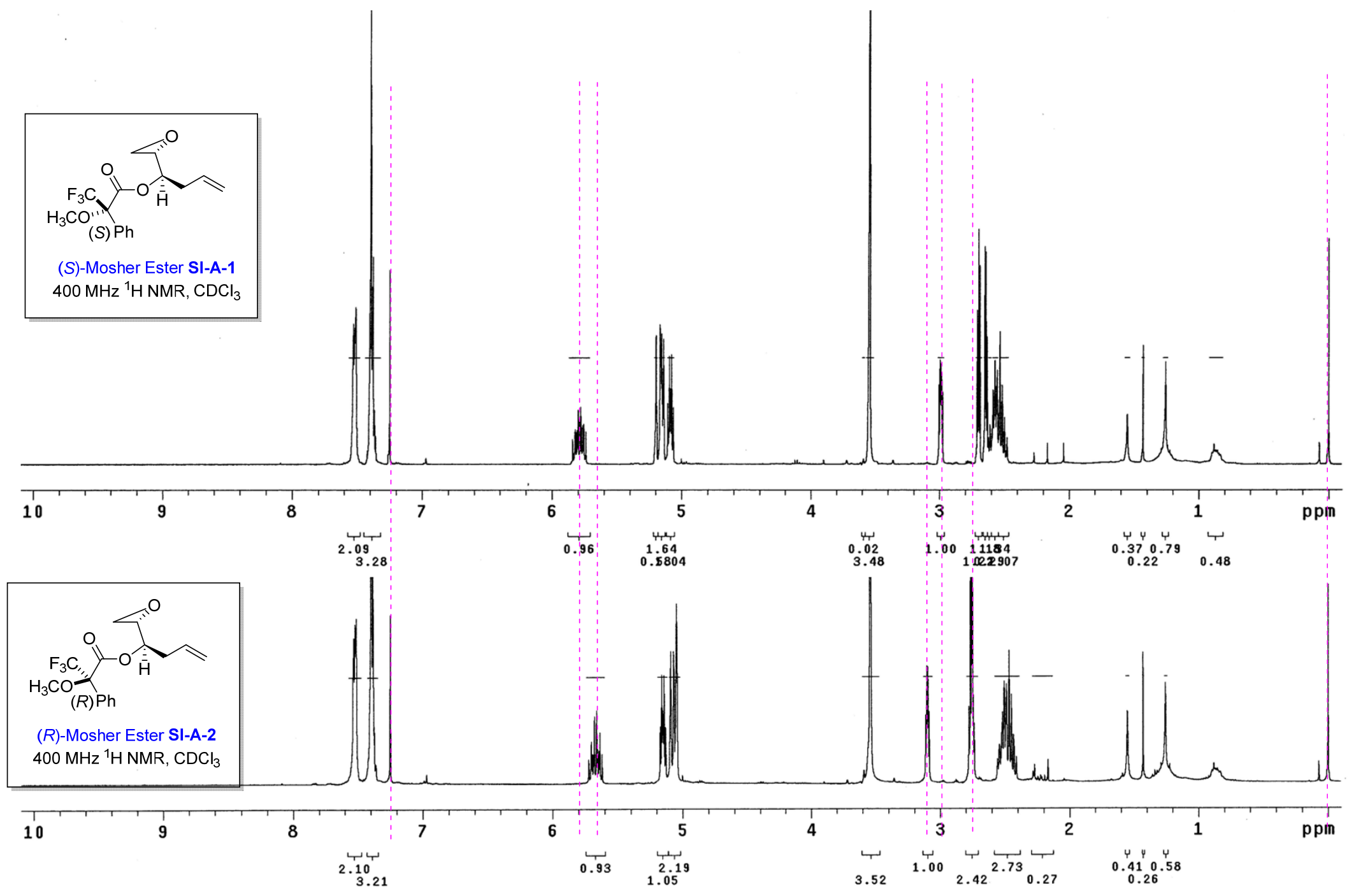




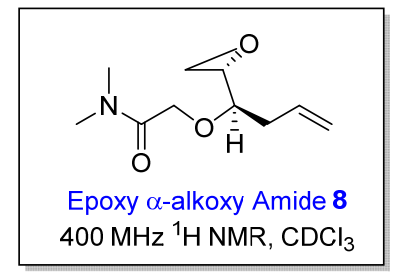

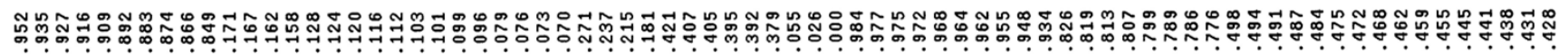
L
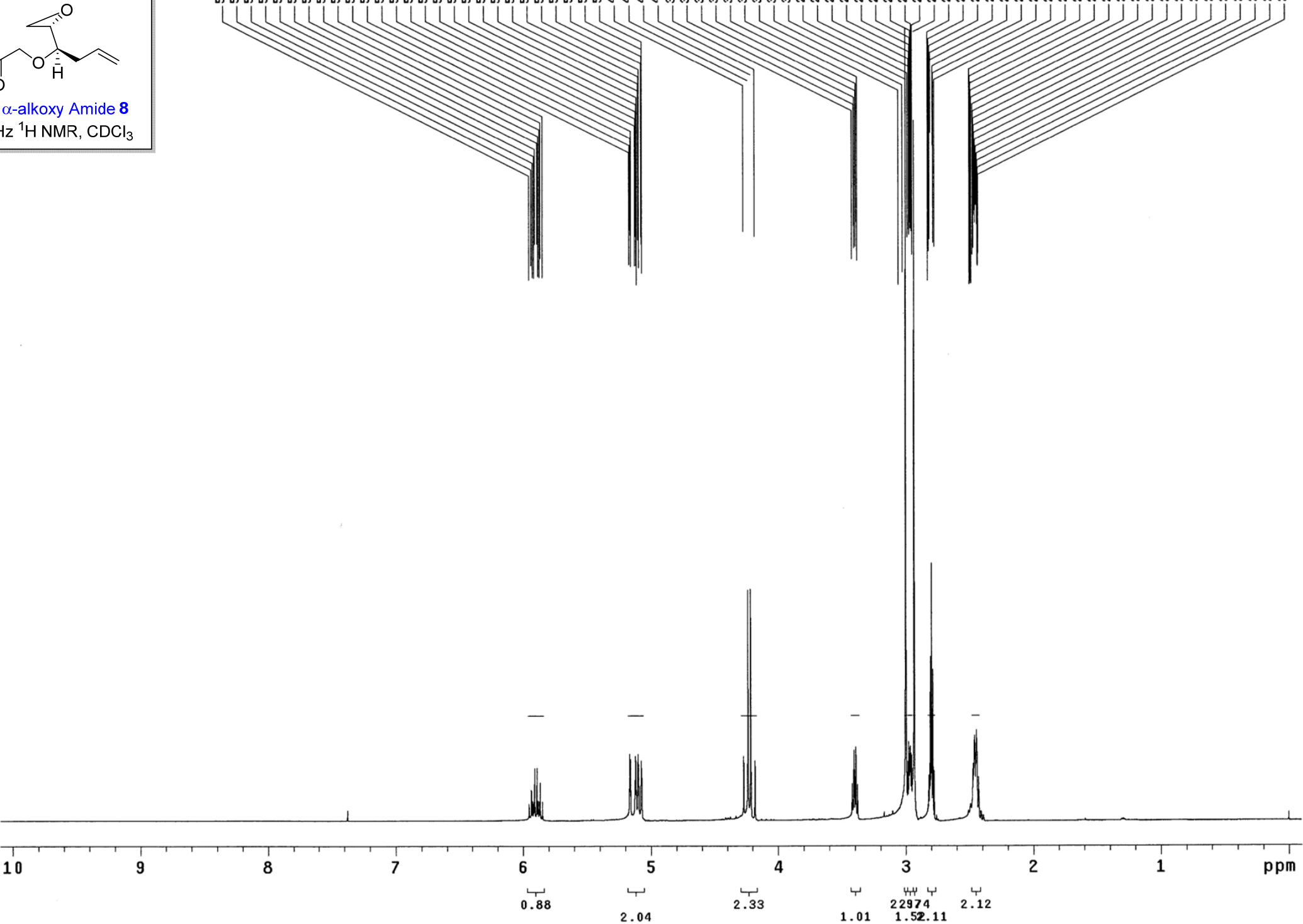

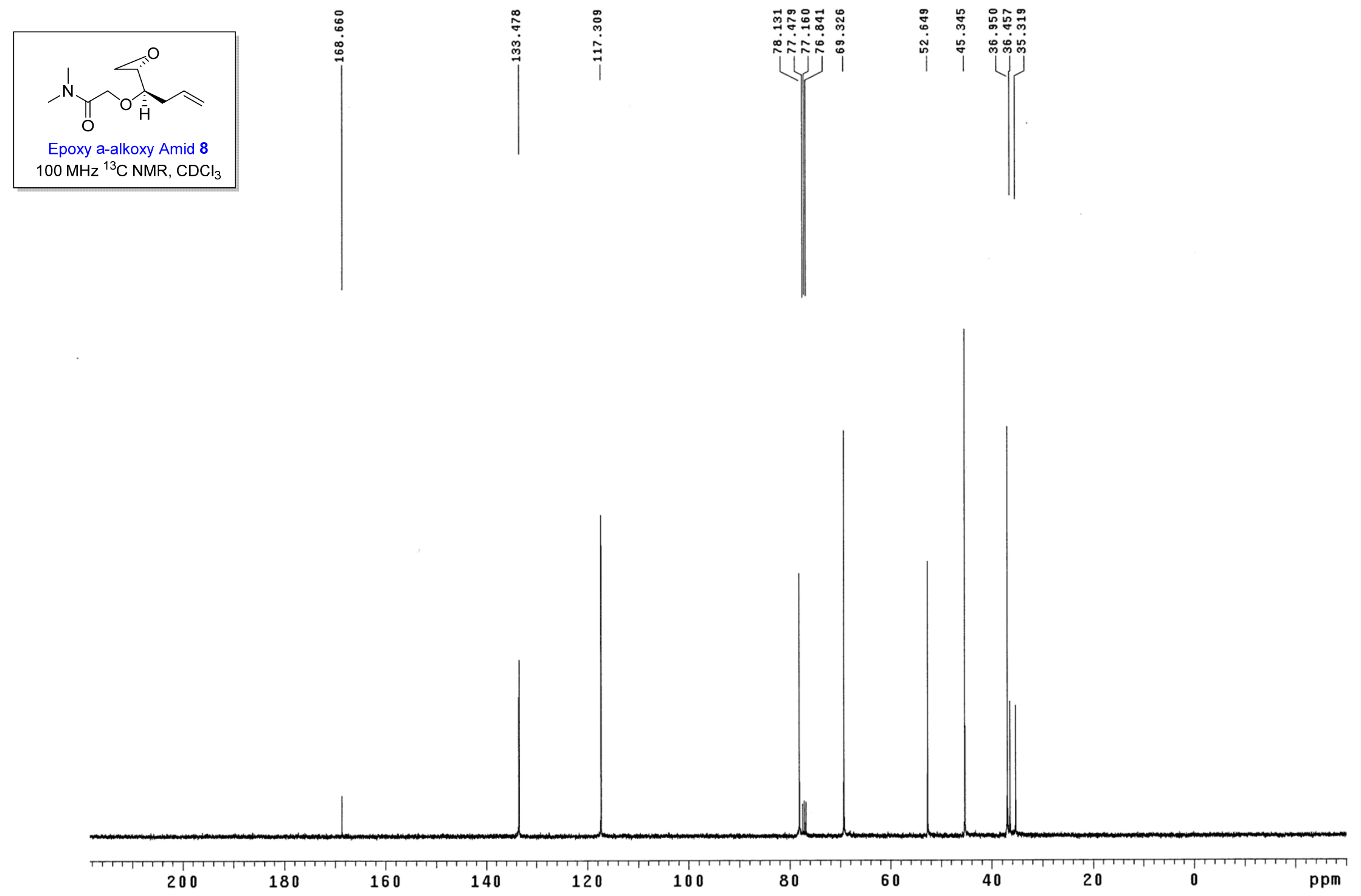


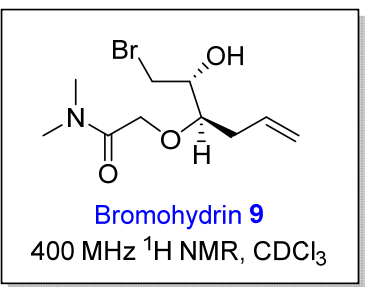

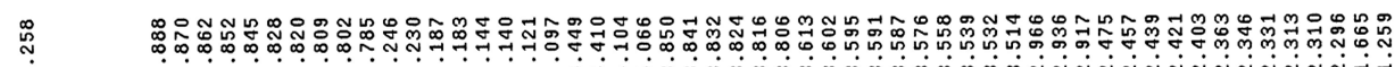

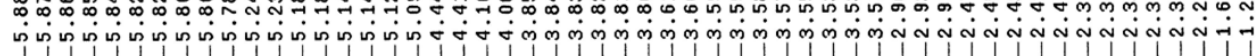

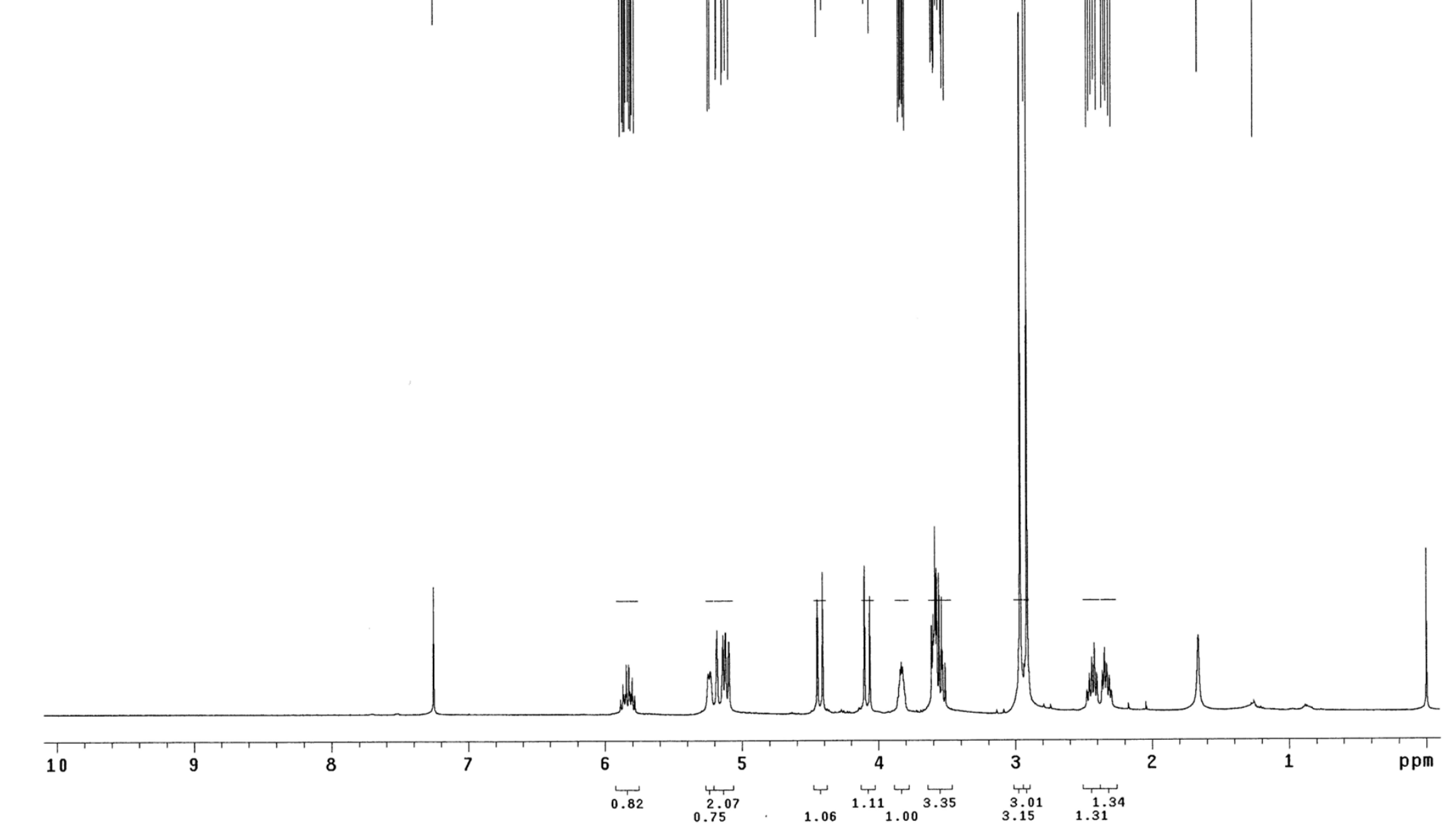




$100 \mathrm{MHz}^{13} \mathrm{C} \mathrm{NMR,} \mathrm{CDCl}_{3}$

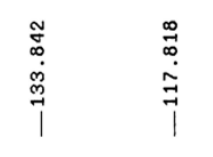

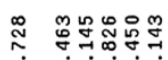

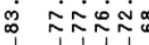

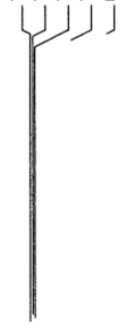

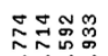

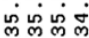

[บ|

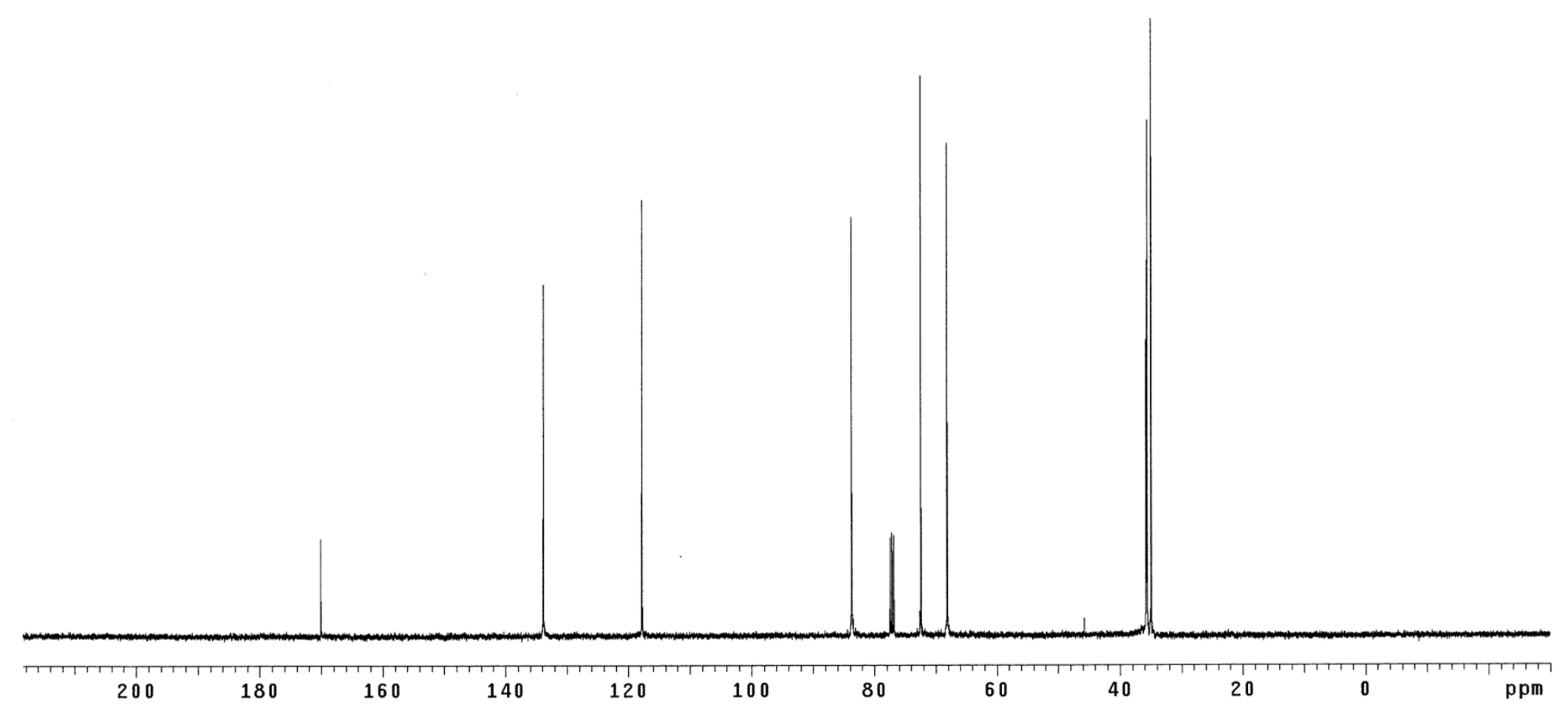




BIPS-protected
Bromo Amide 6
$400 \mathrm{MHz}{ }^{1} \mathrm{H}$ NMR, $\mathrm{CDCl}_{3}$
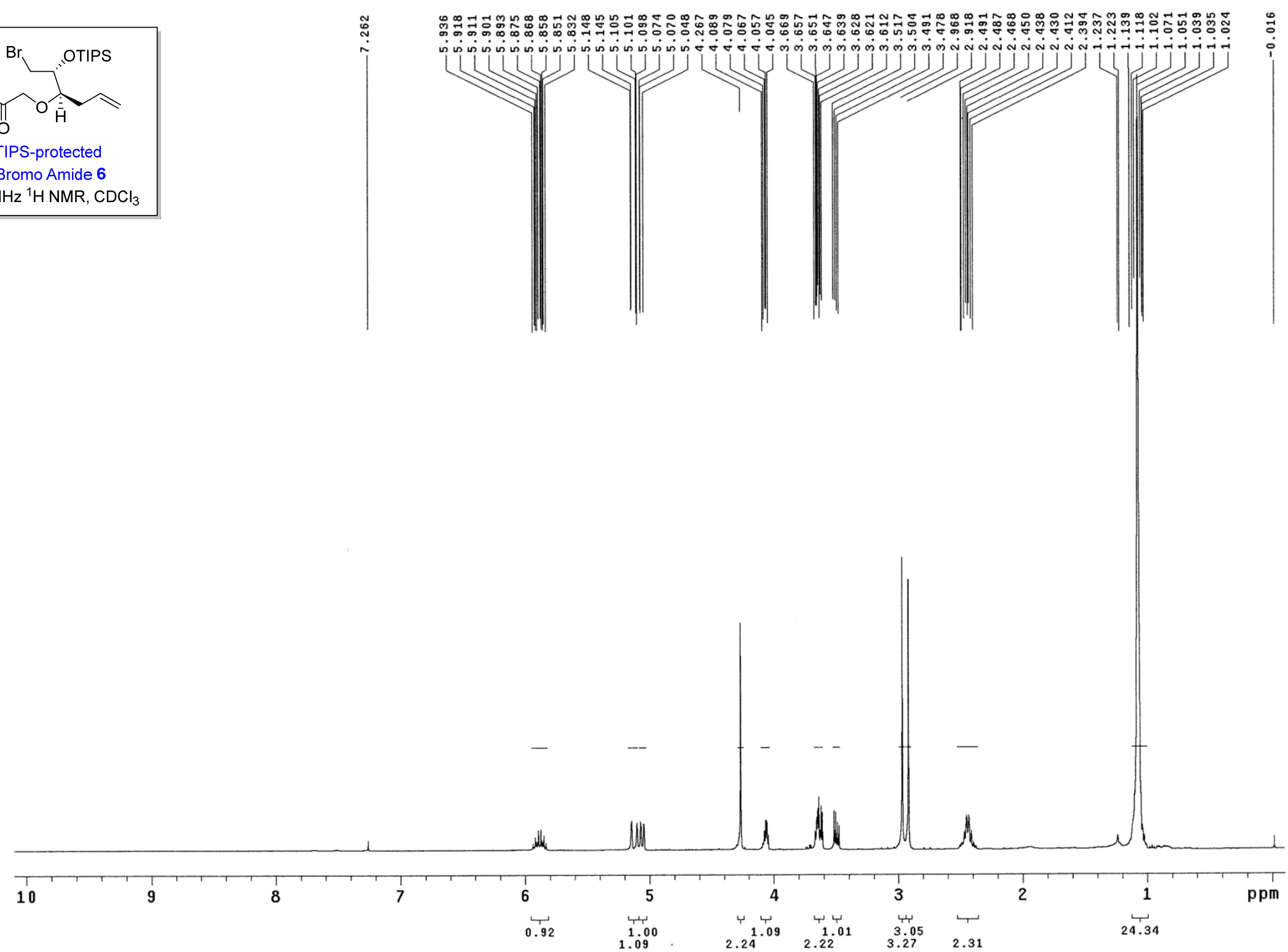


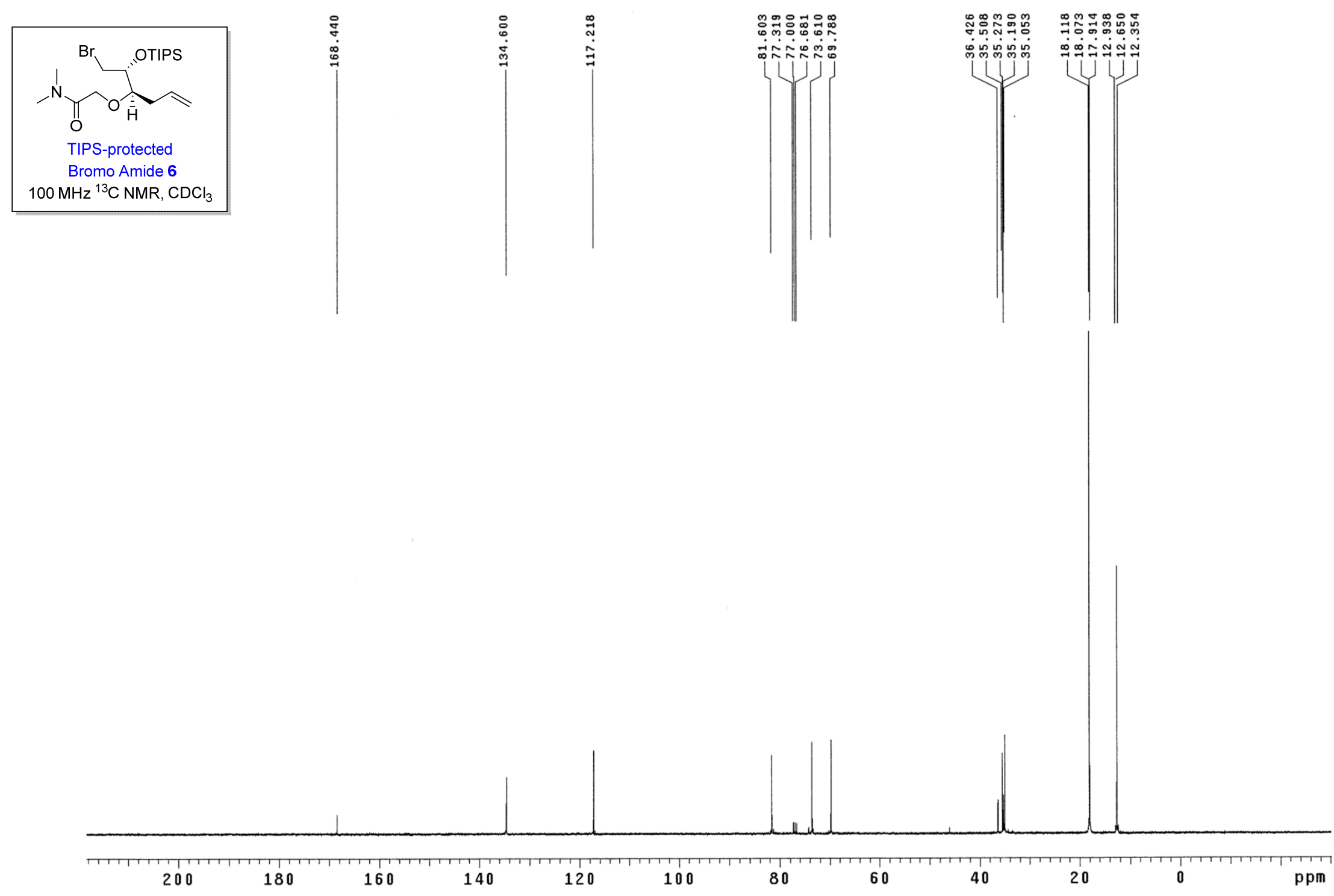




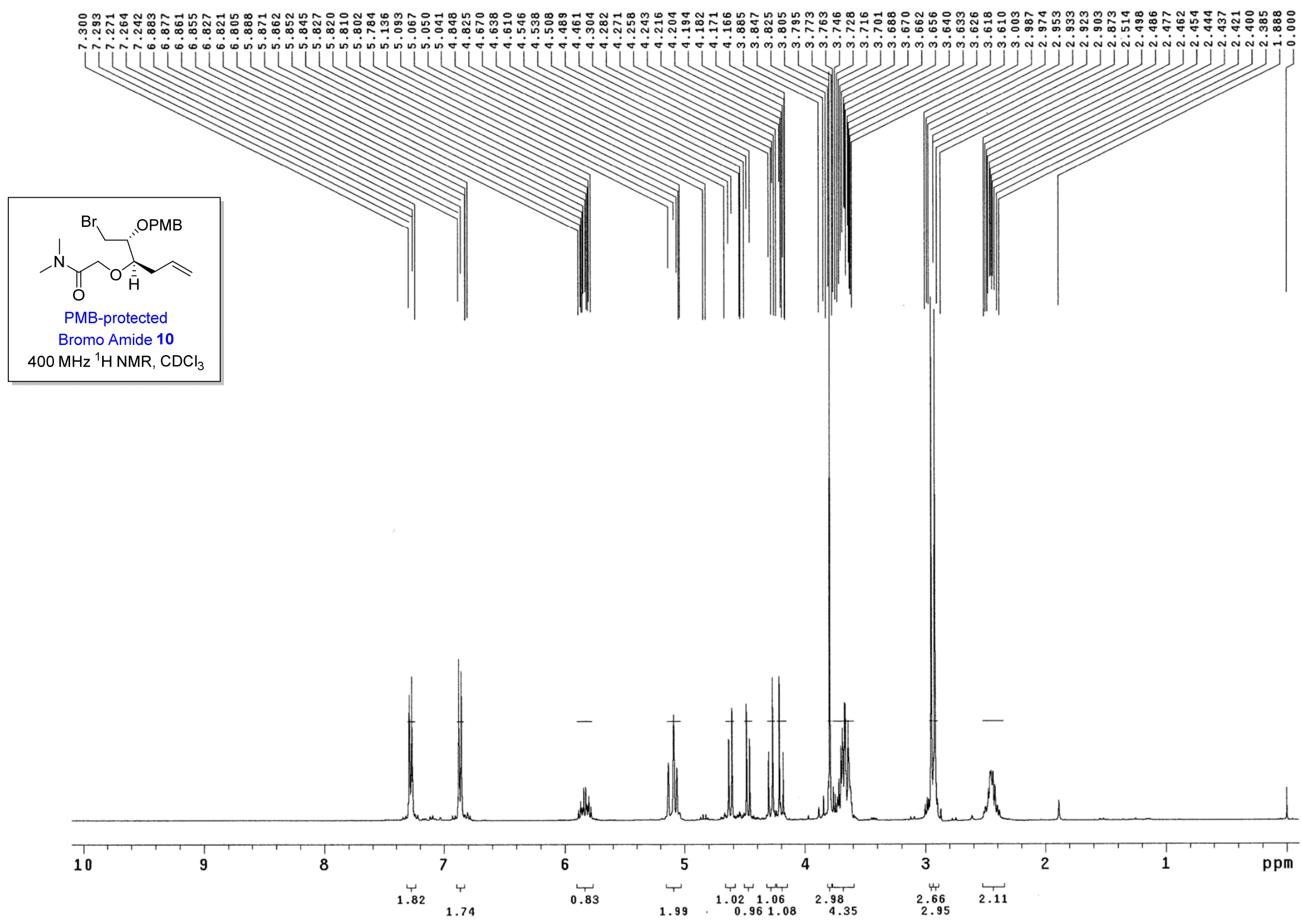




BMB-protected
Bromo Amide 10
$100 \mathrm{MHz}{ }^{13} \mathrm{C}$ NMR, $\mathrm{CDCl}_{3}$
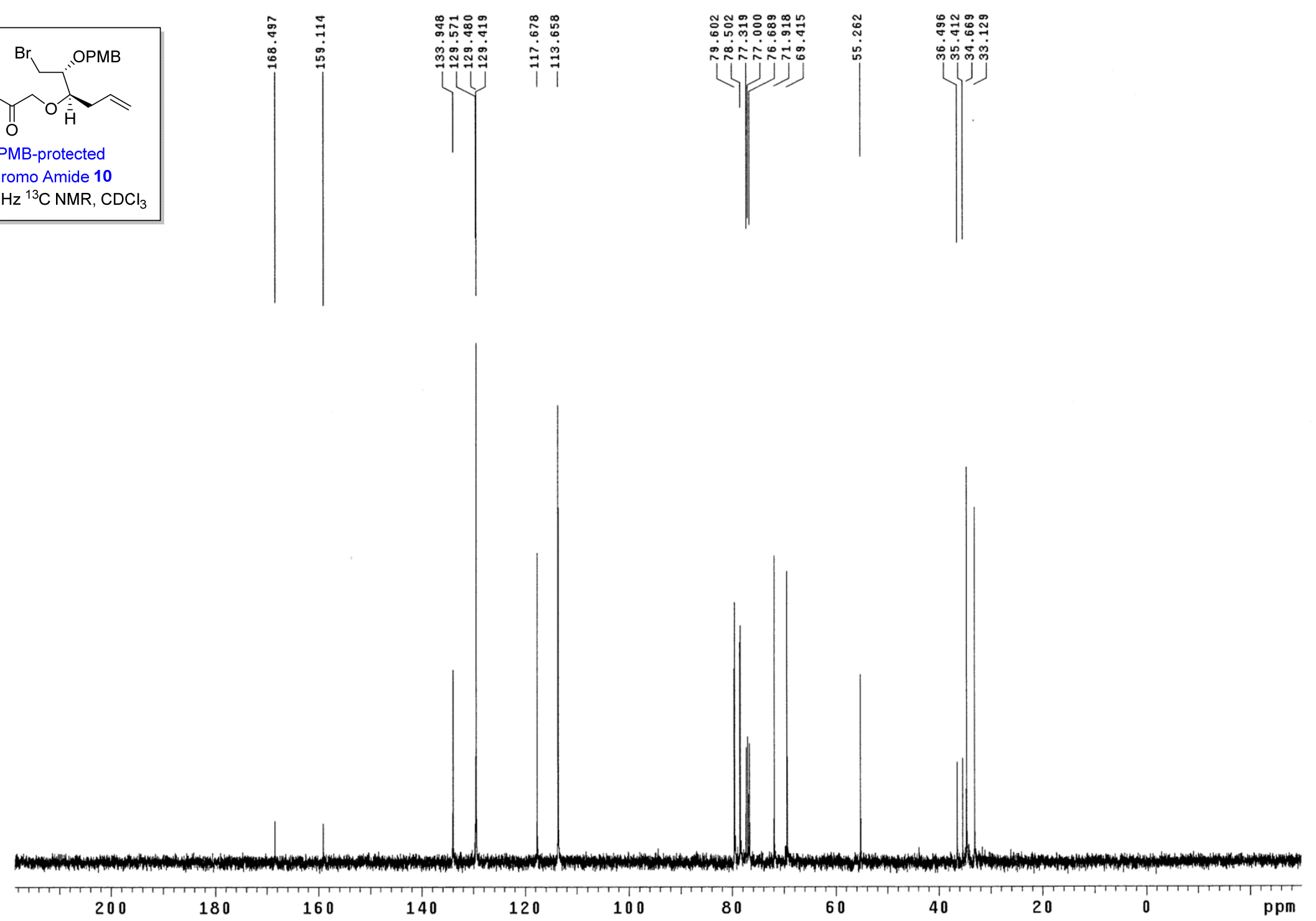


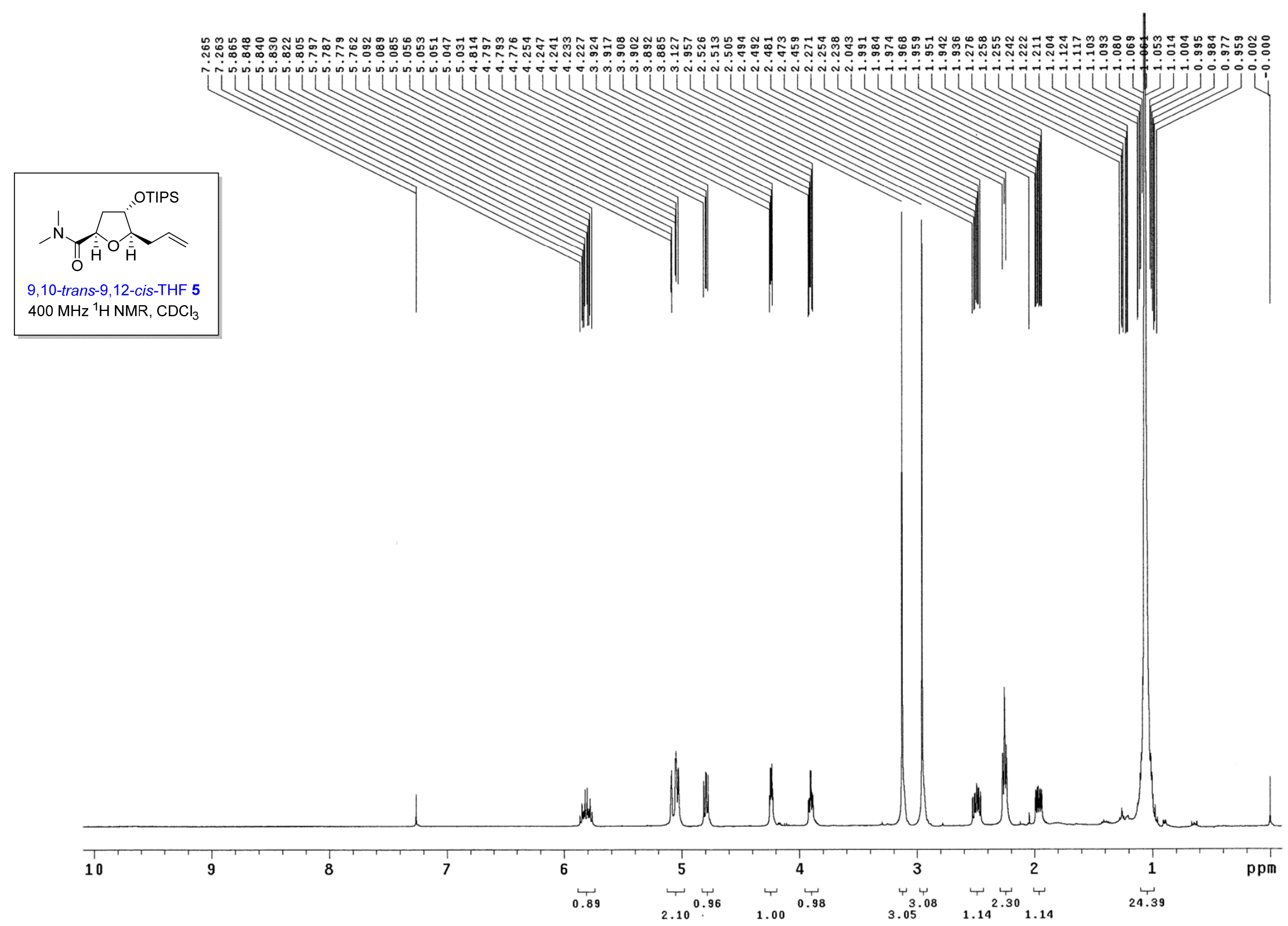




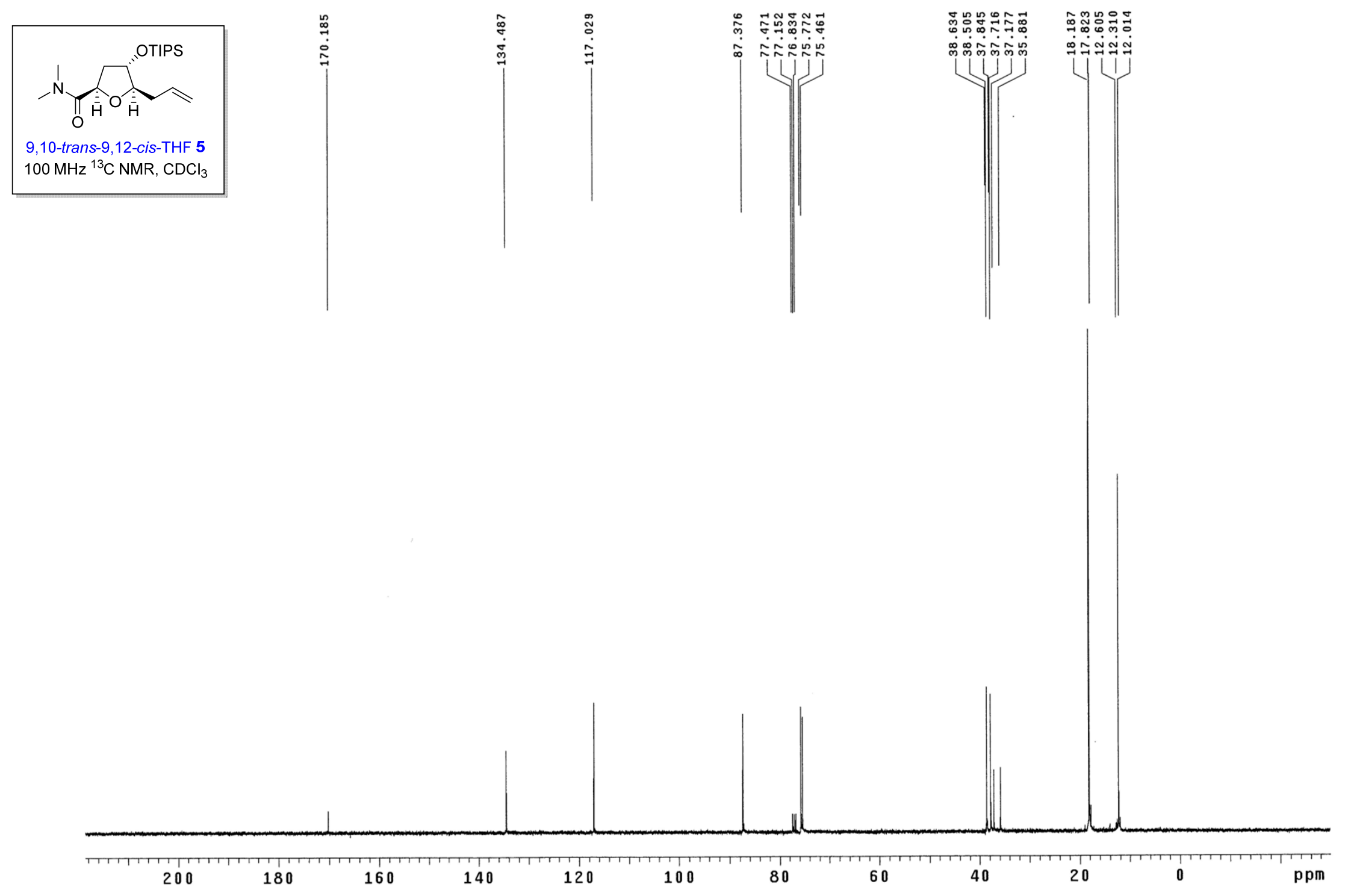




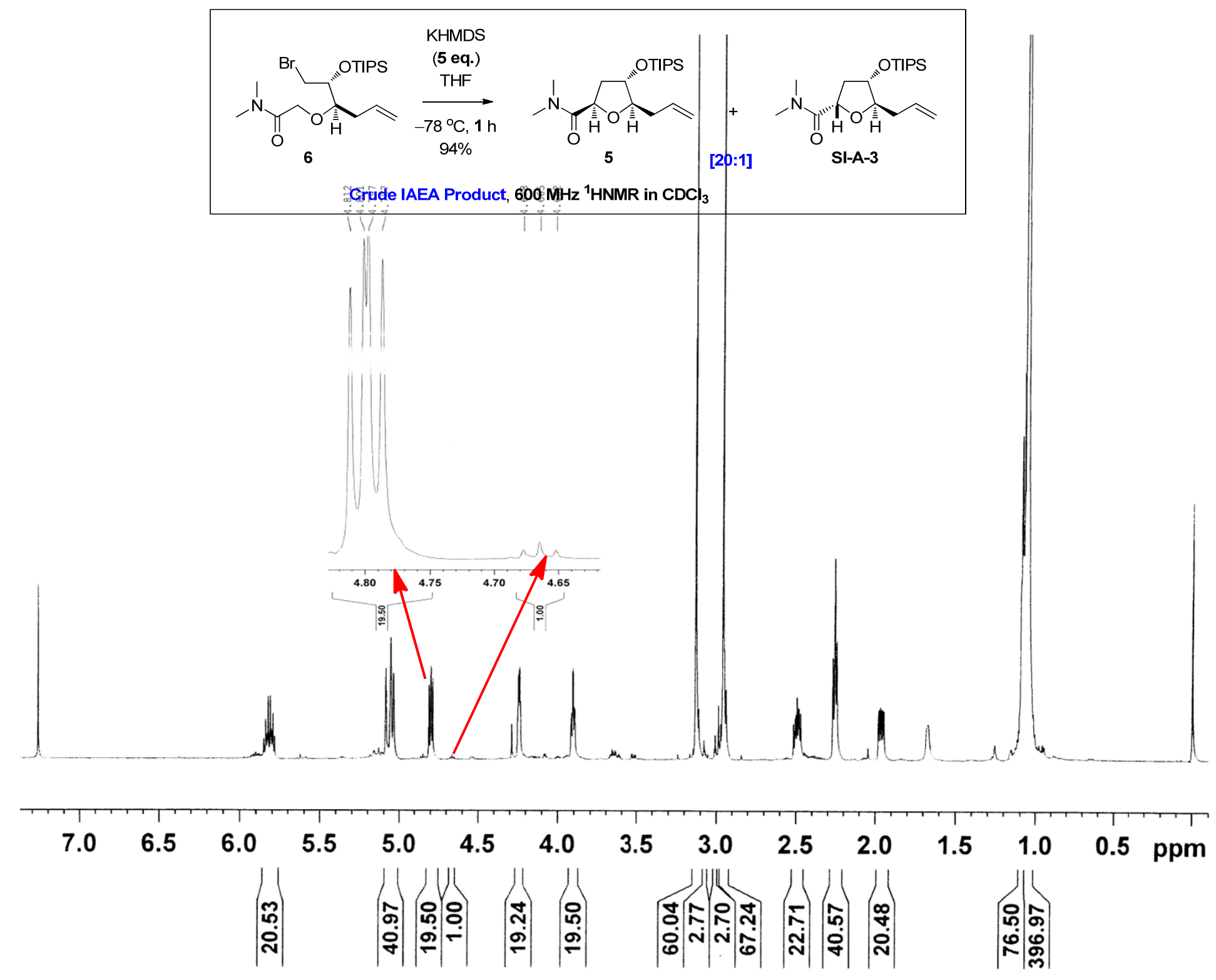




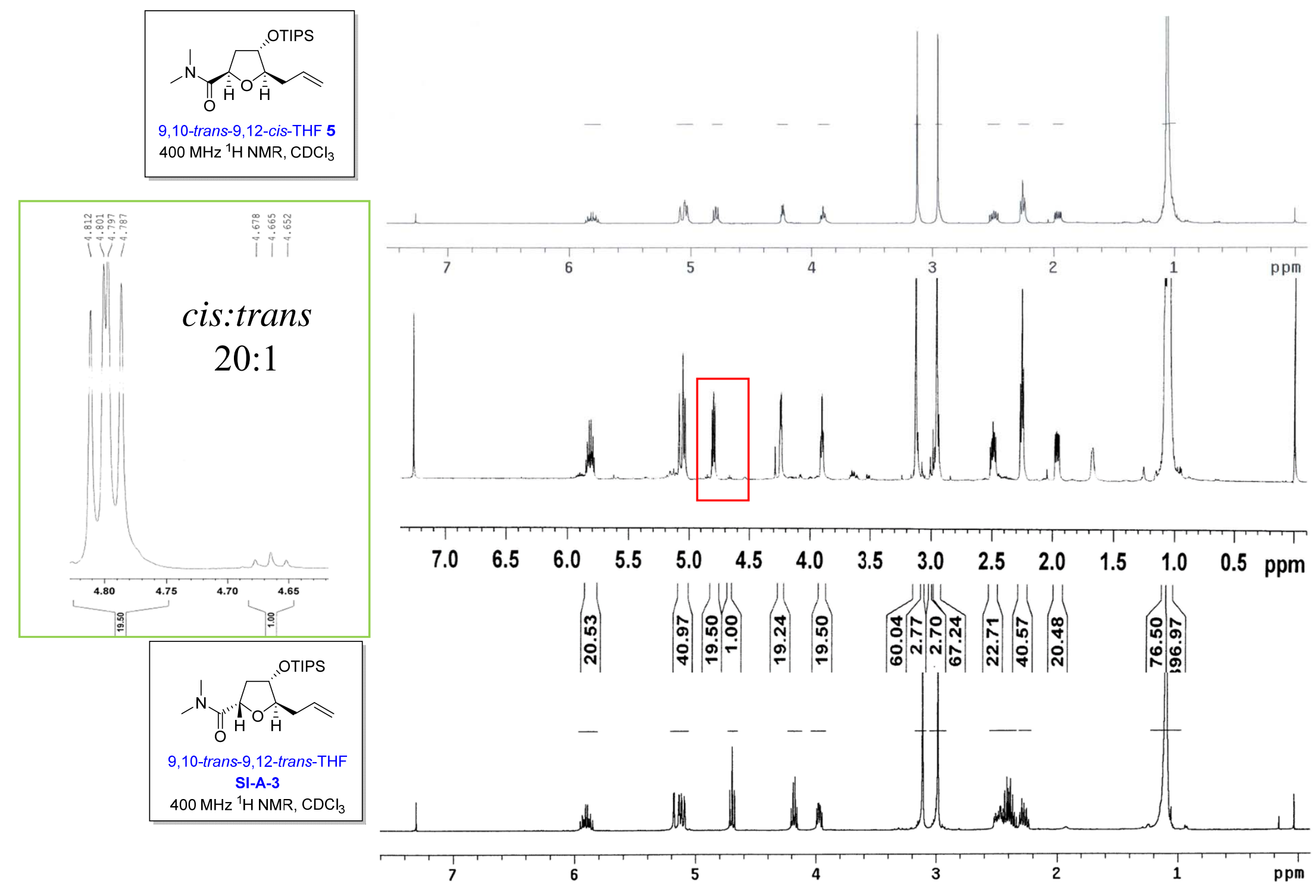




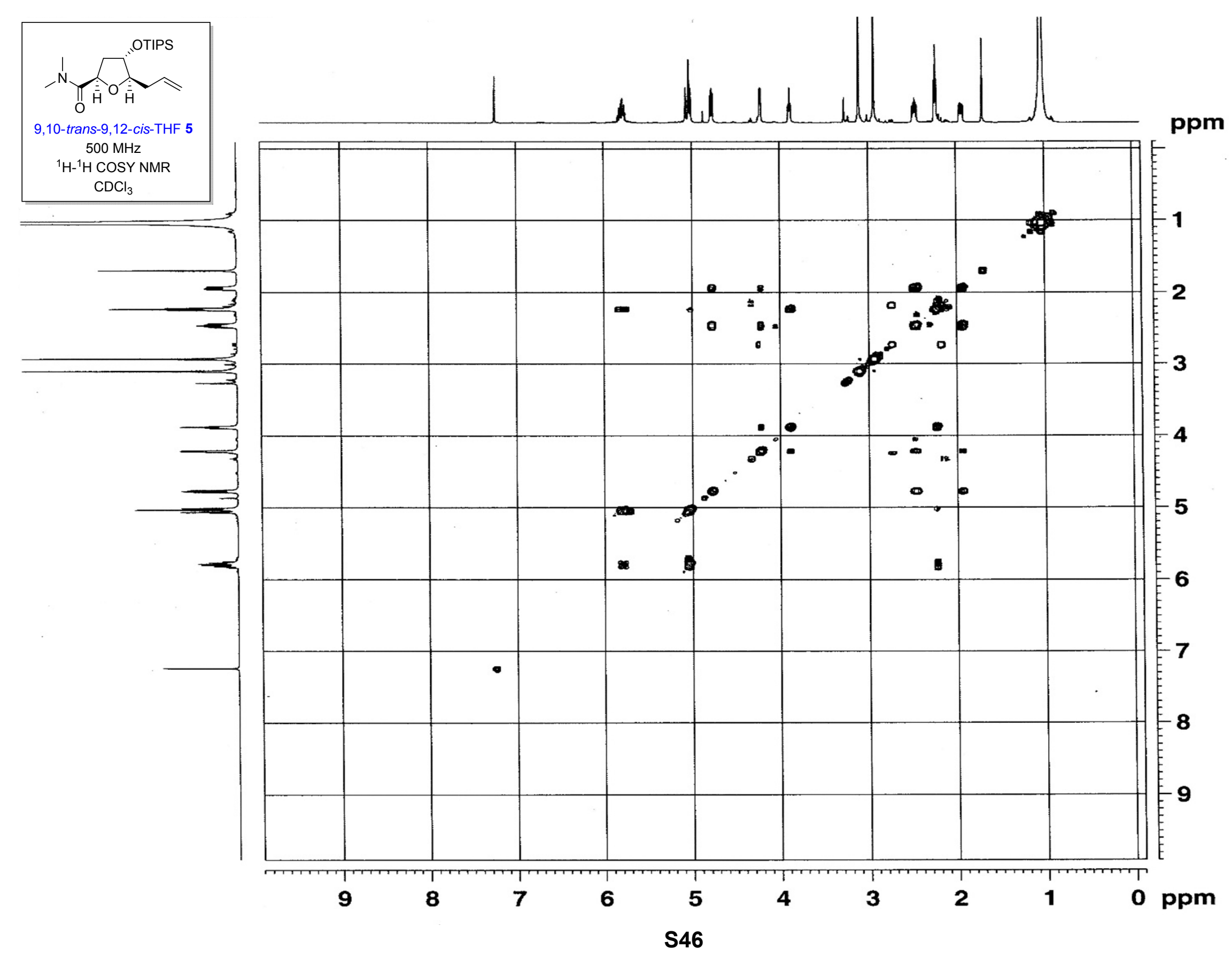




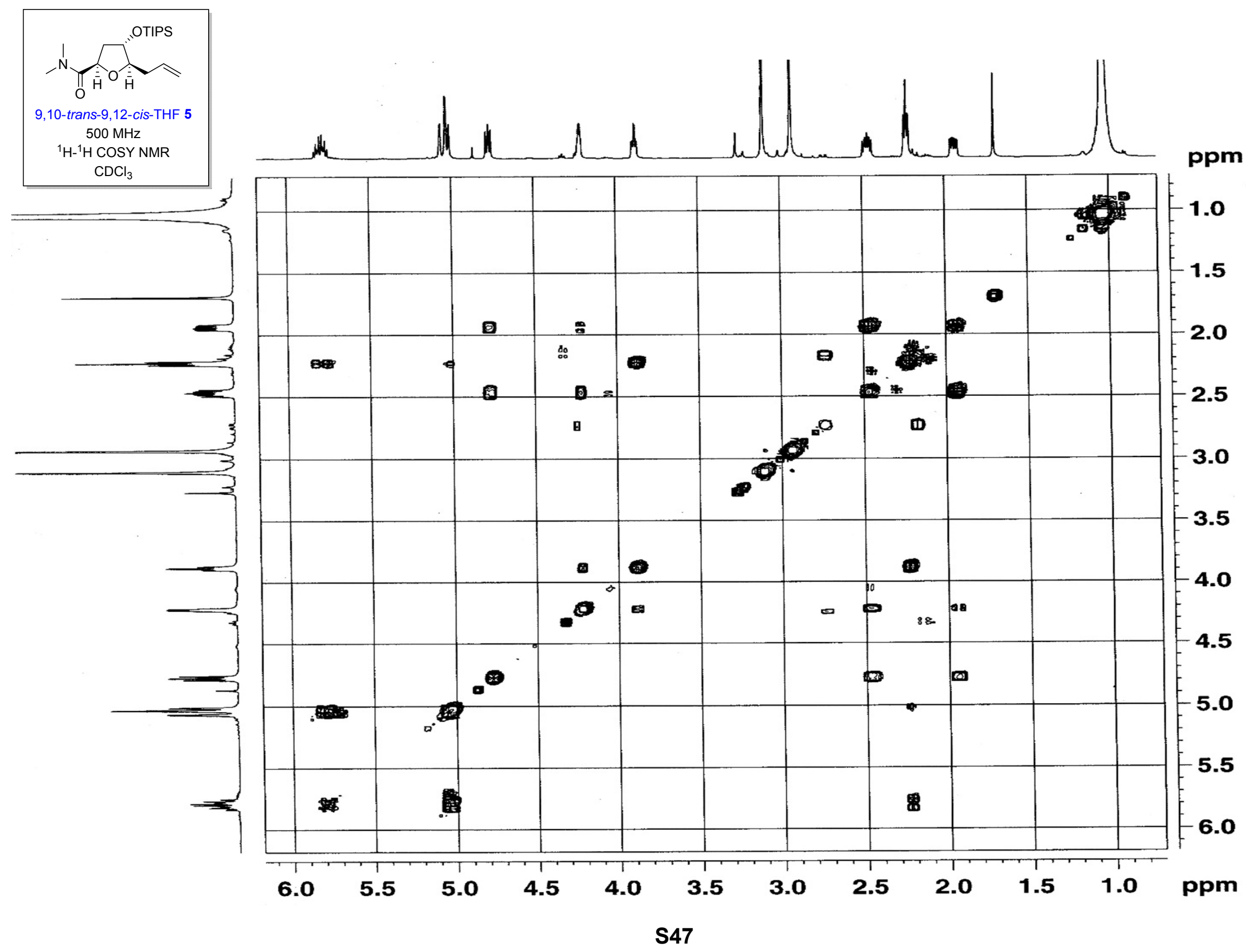




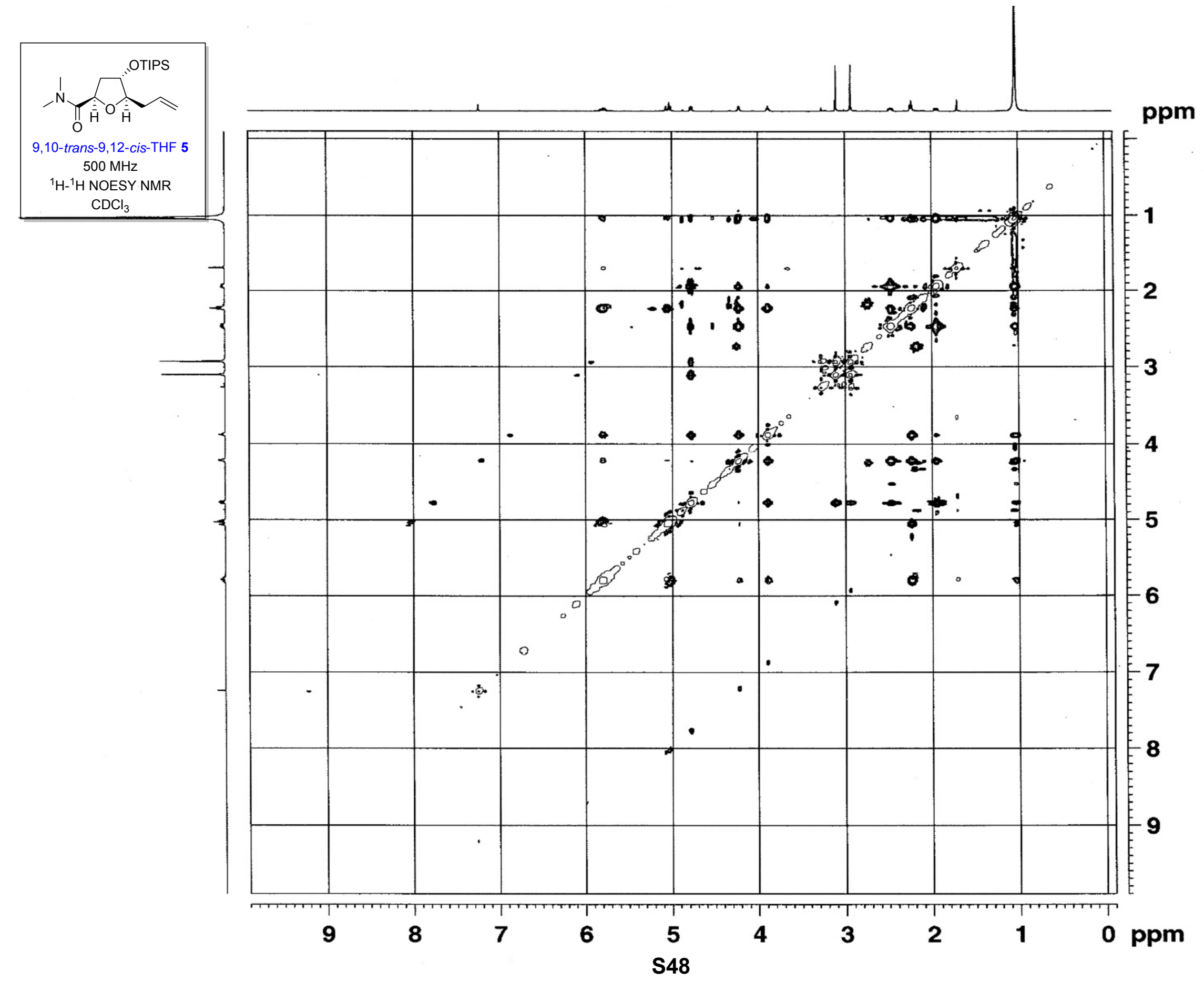




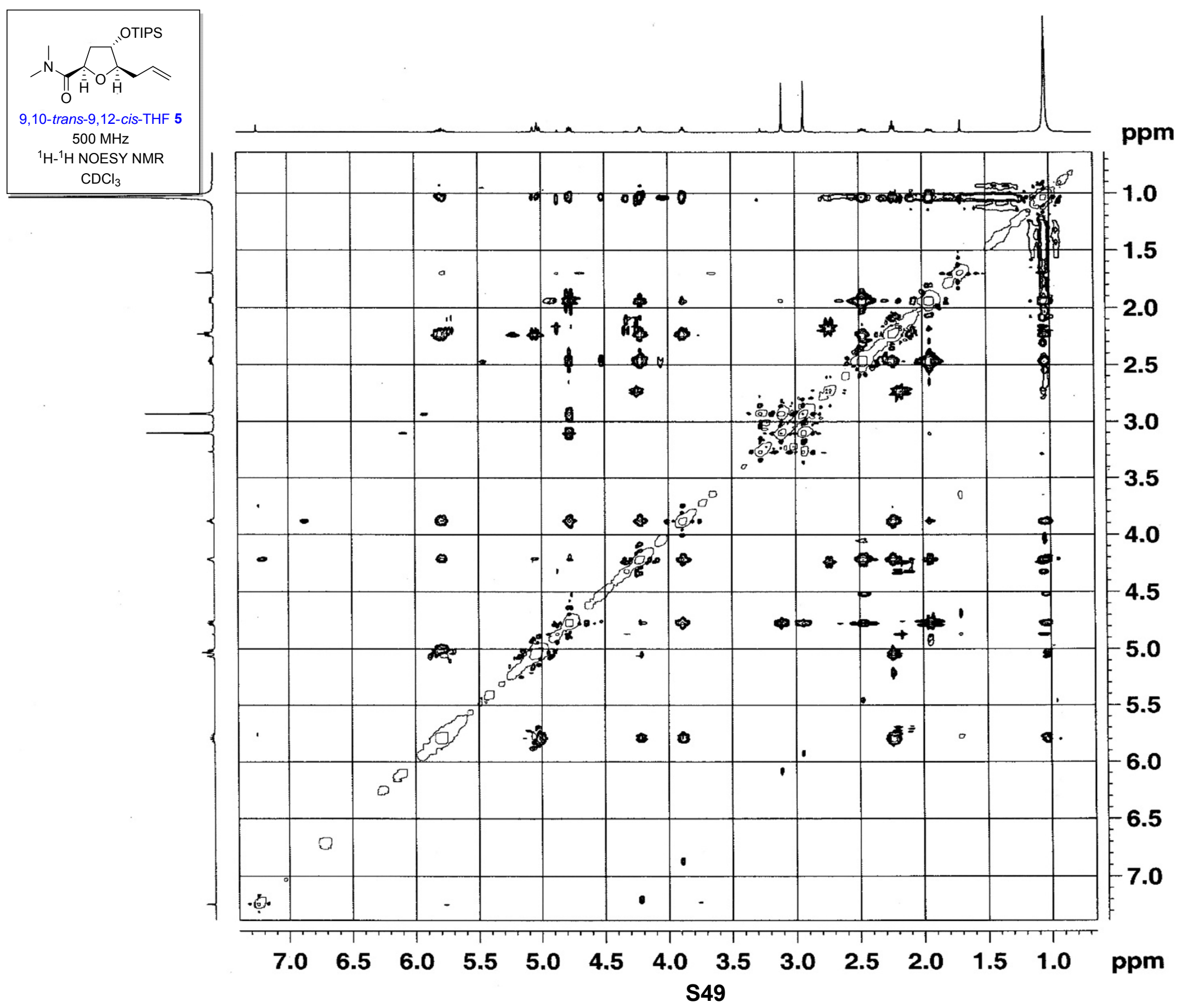



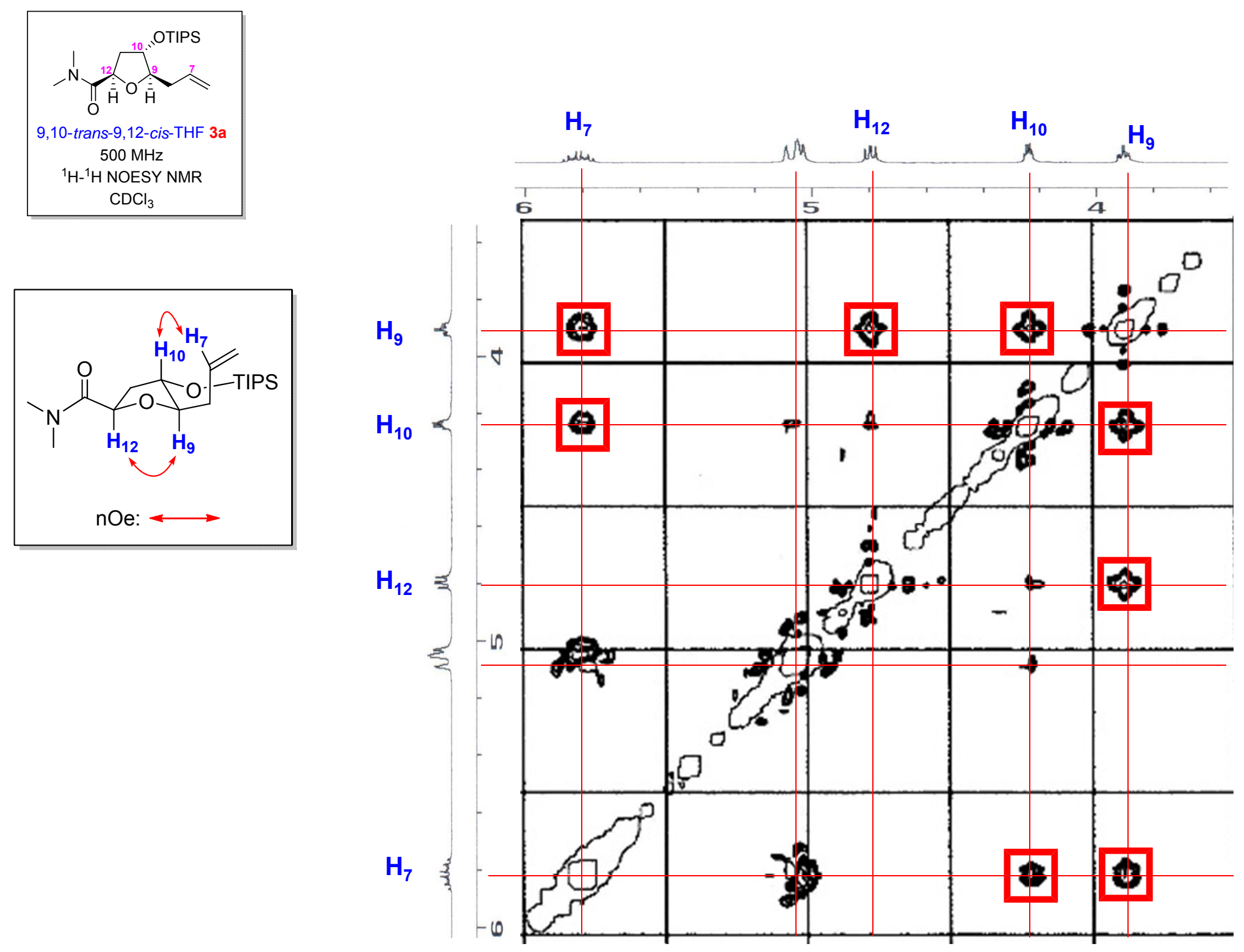


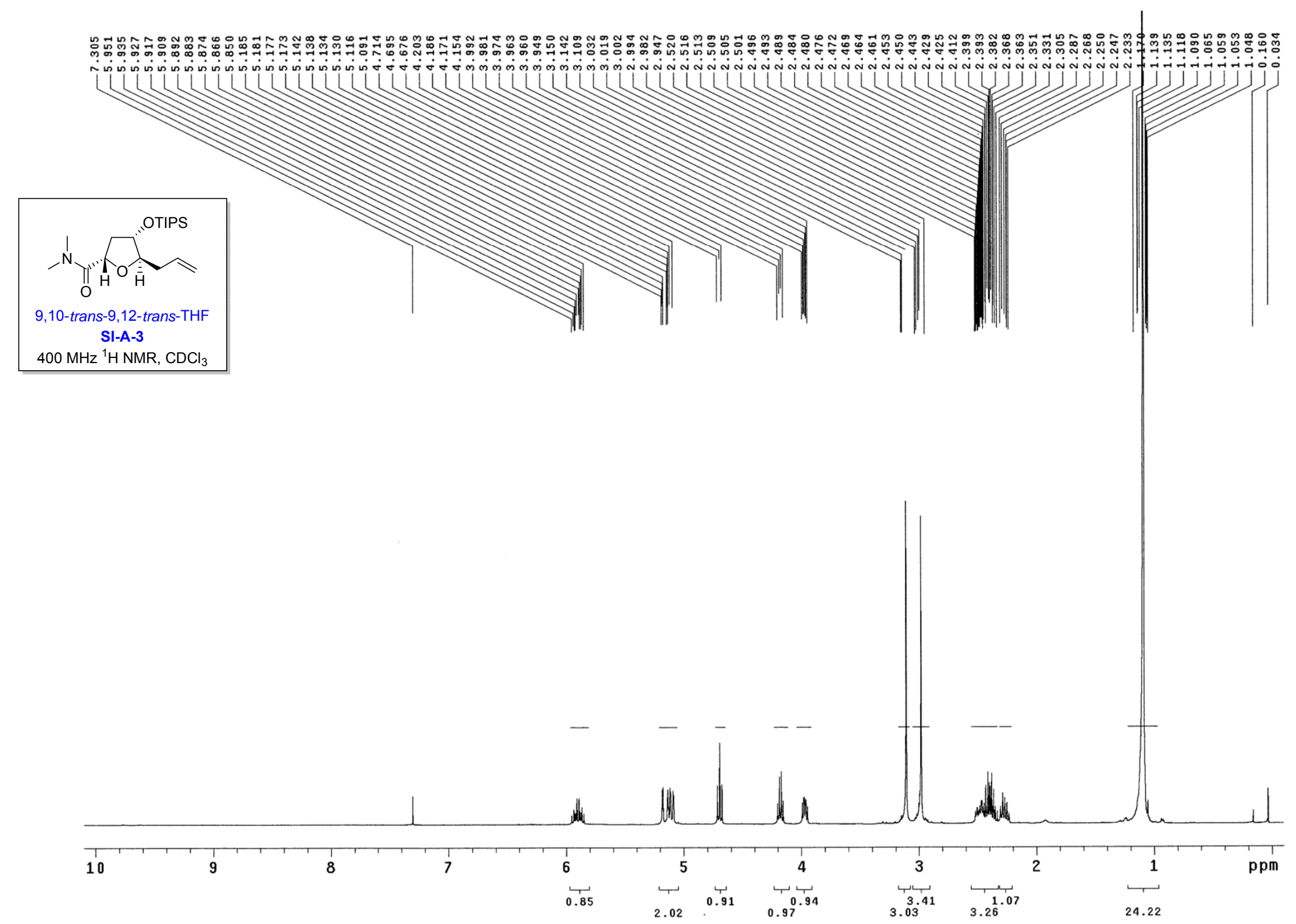




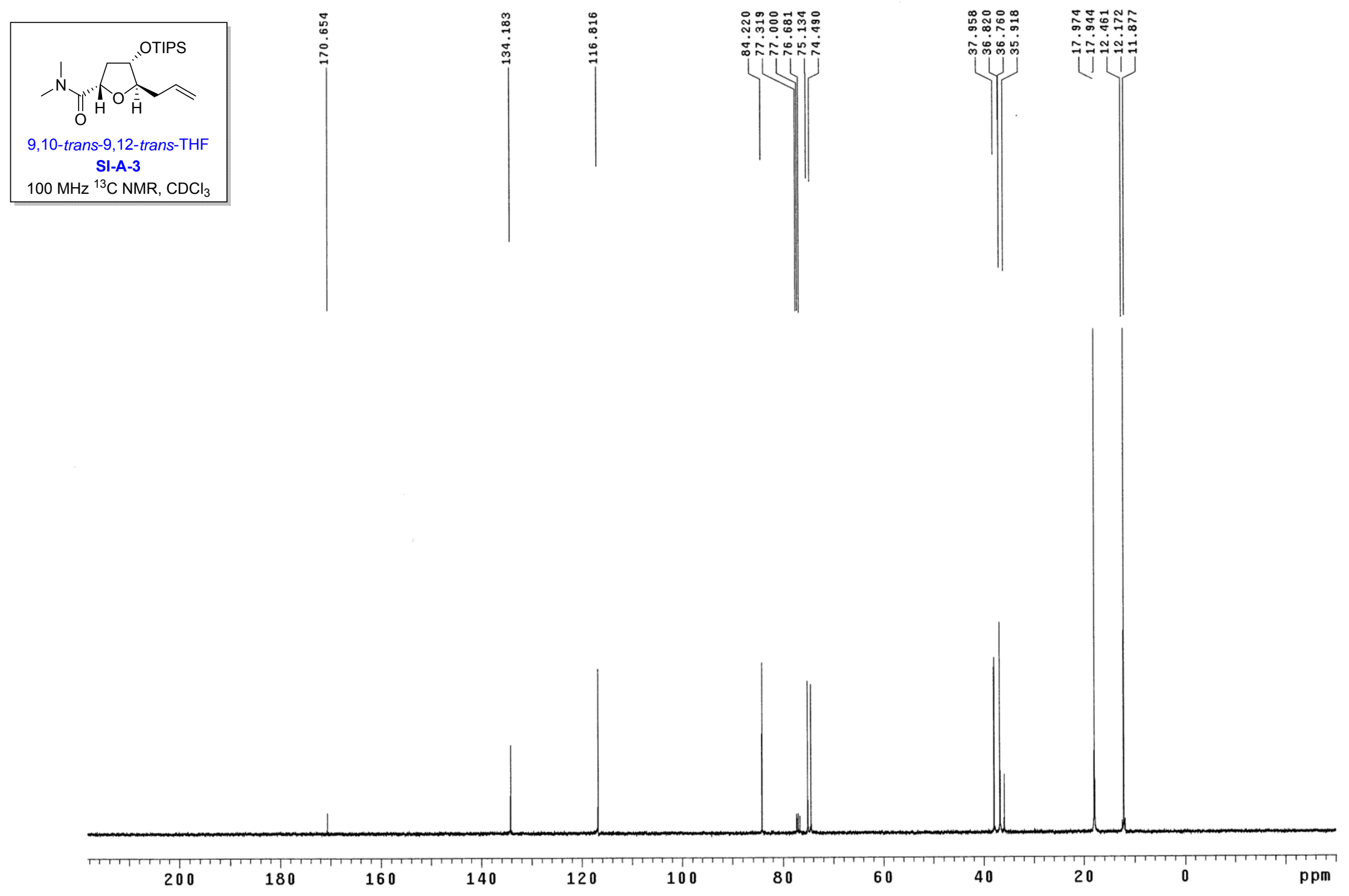




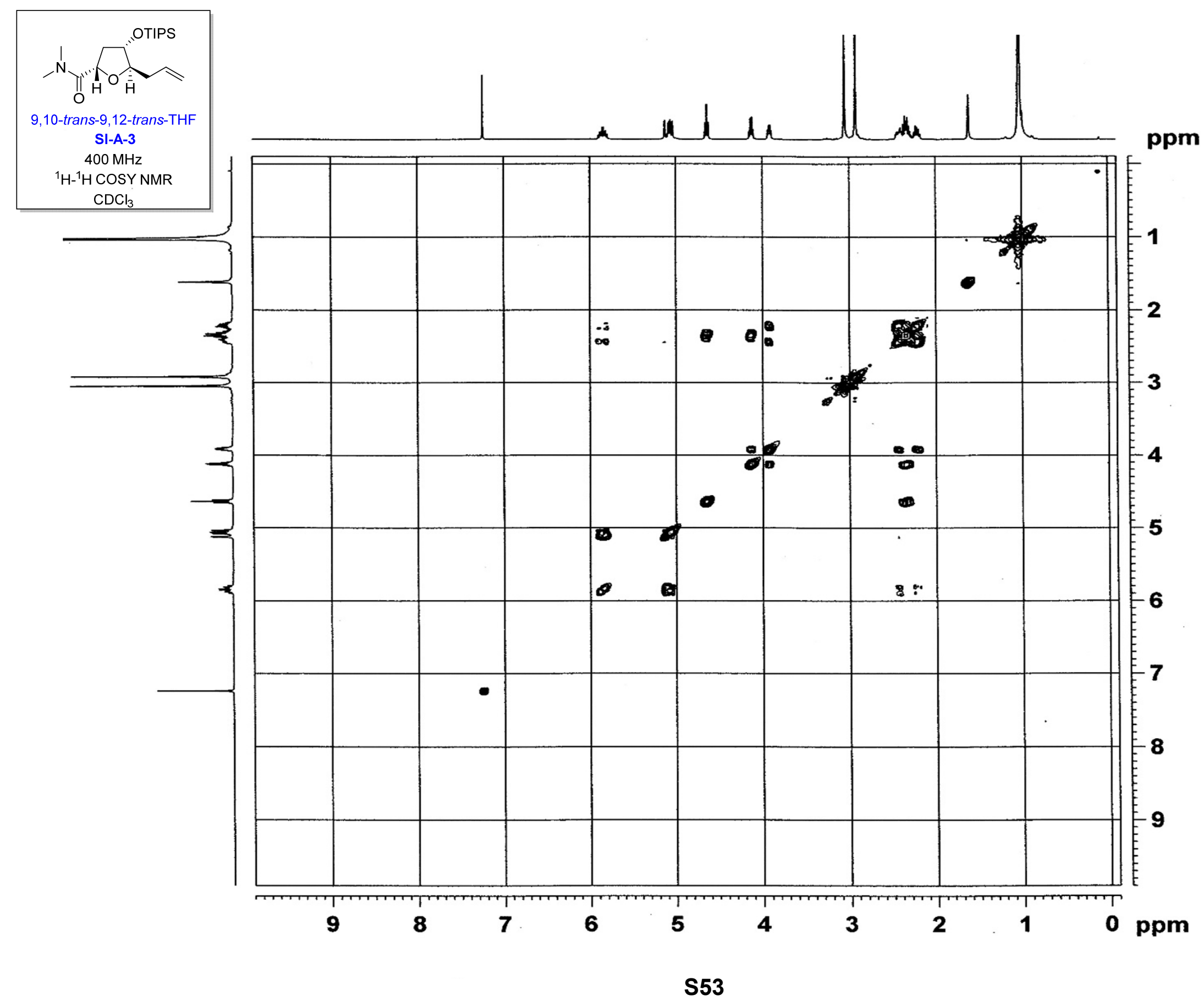




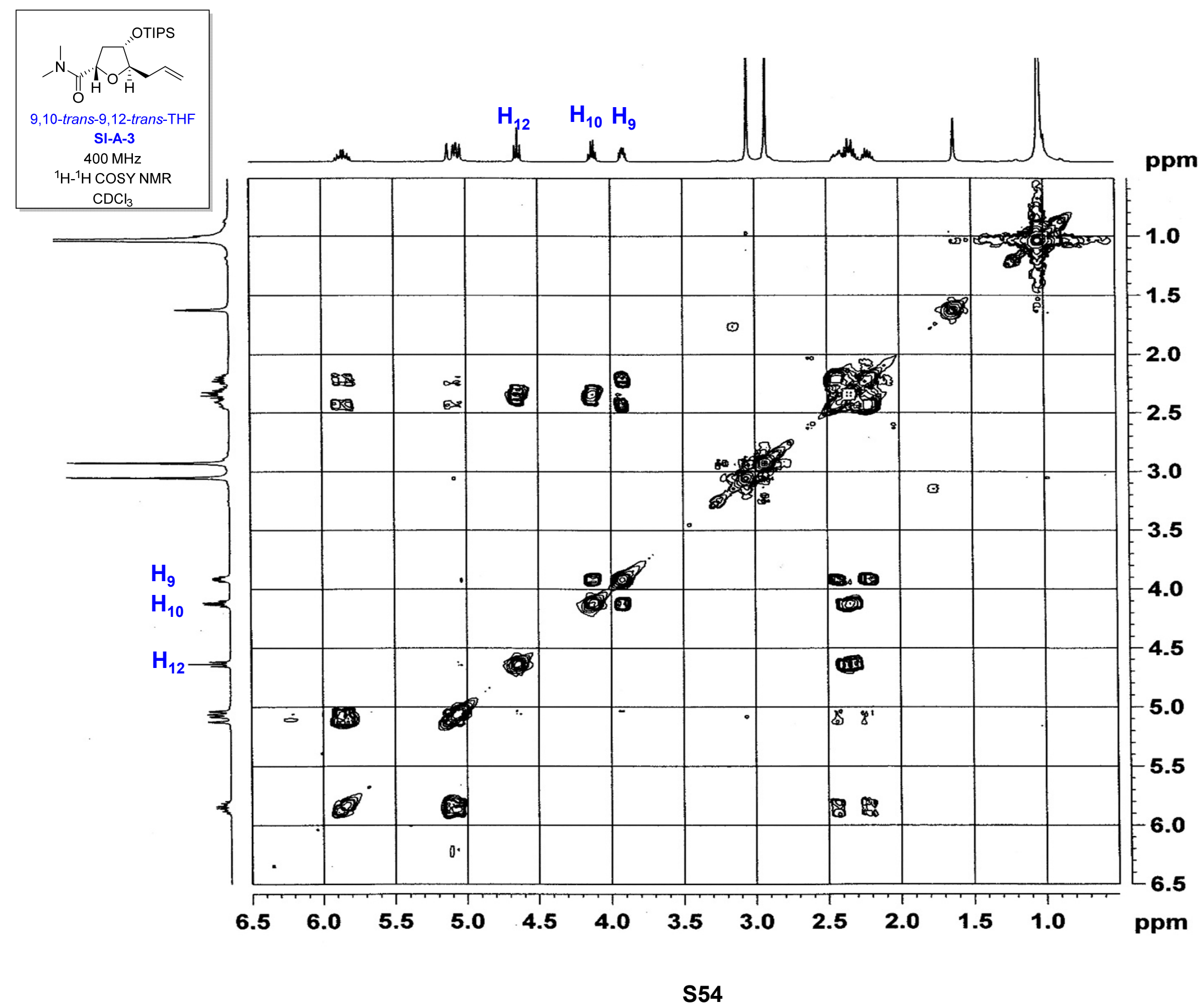




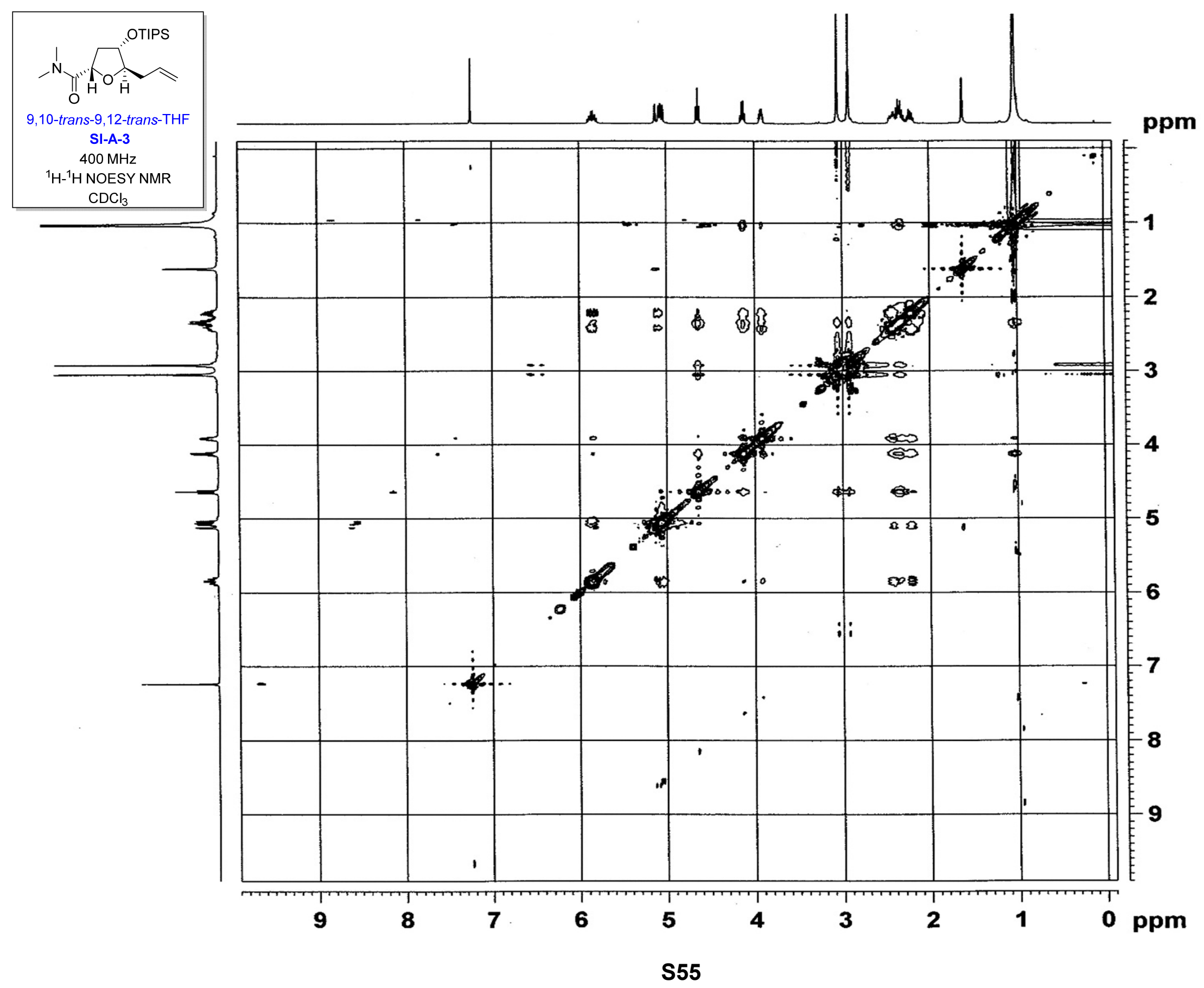




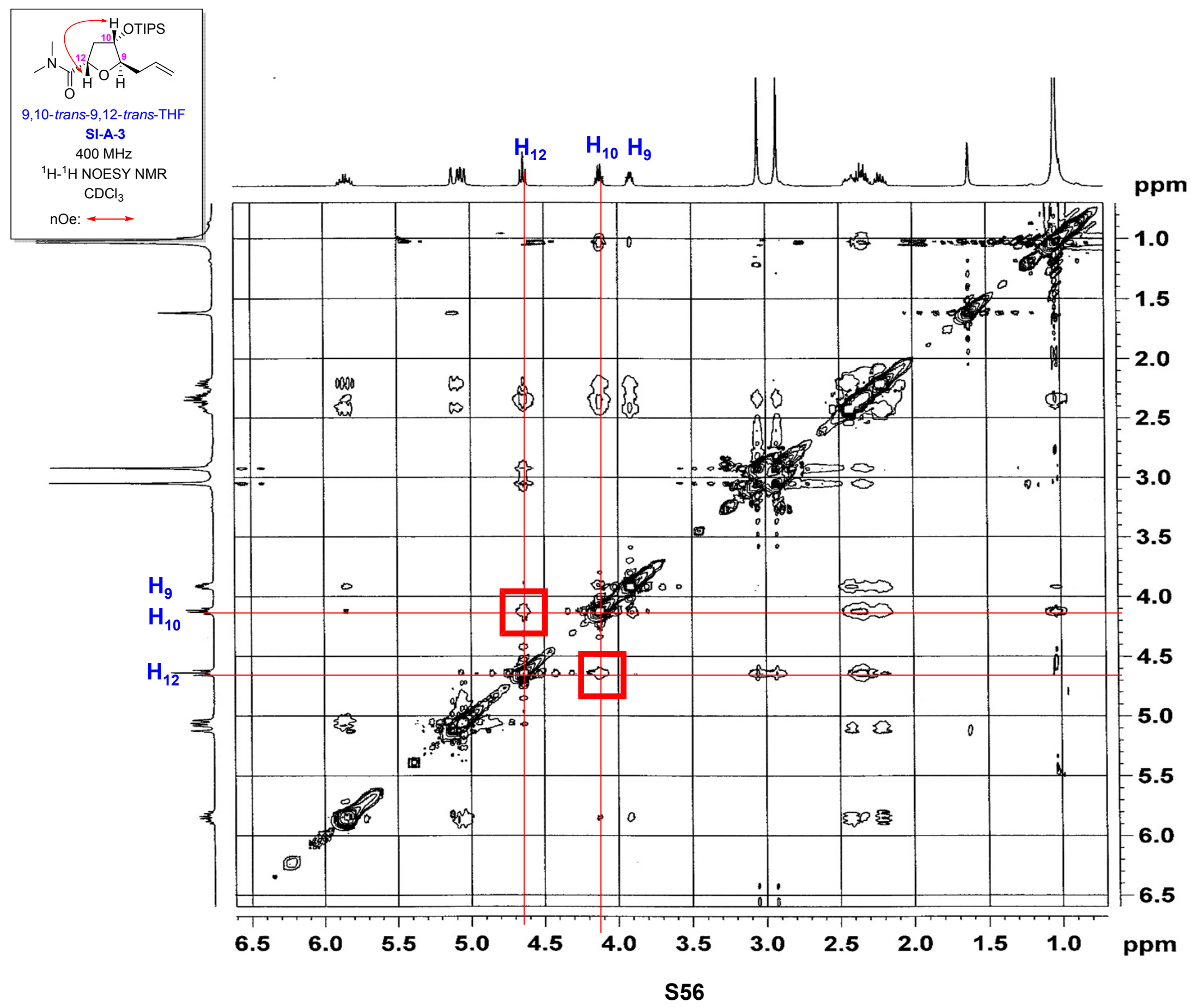




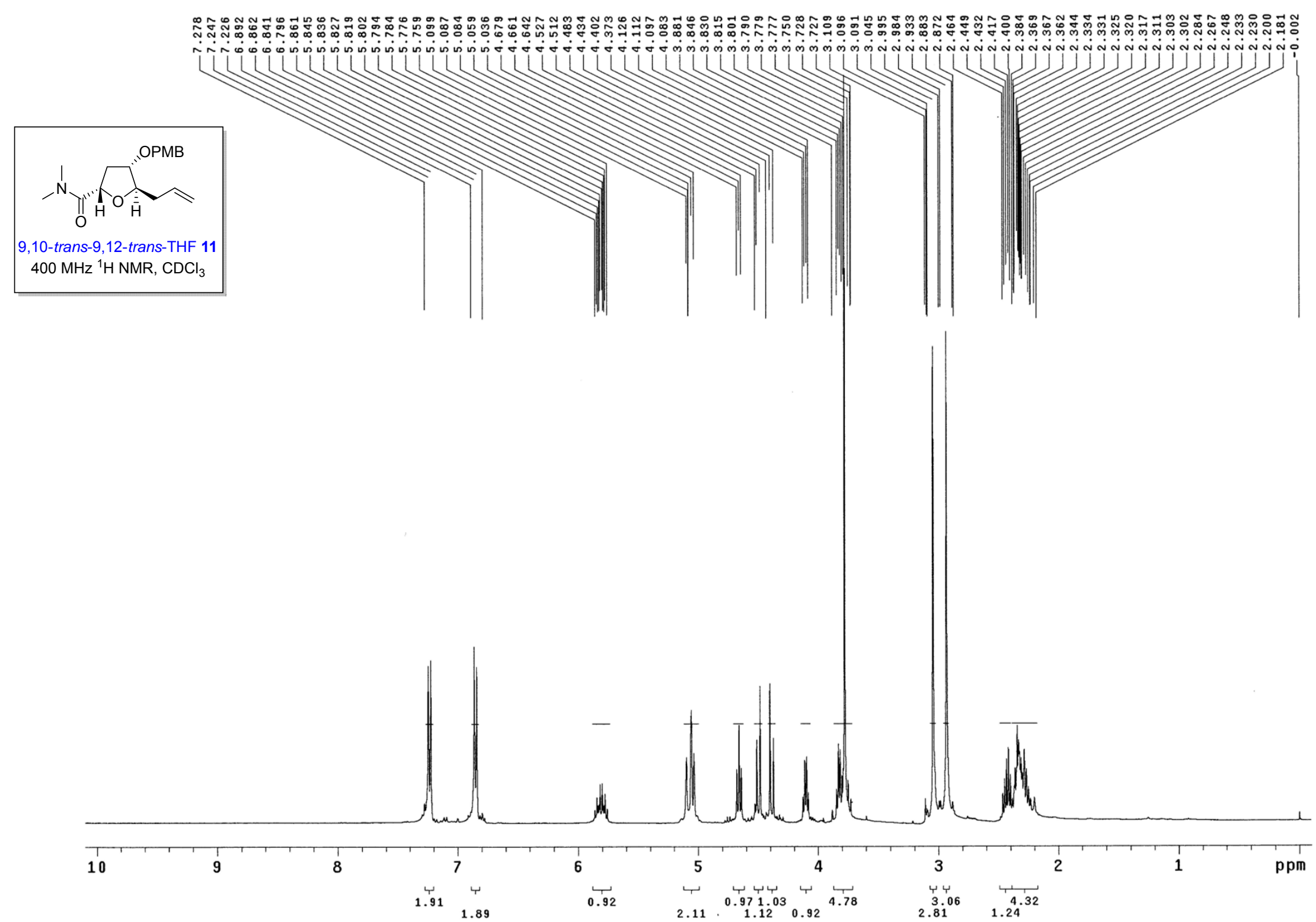




(10-trans-9,12-trans-THF 11
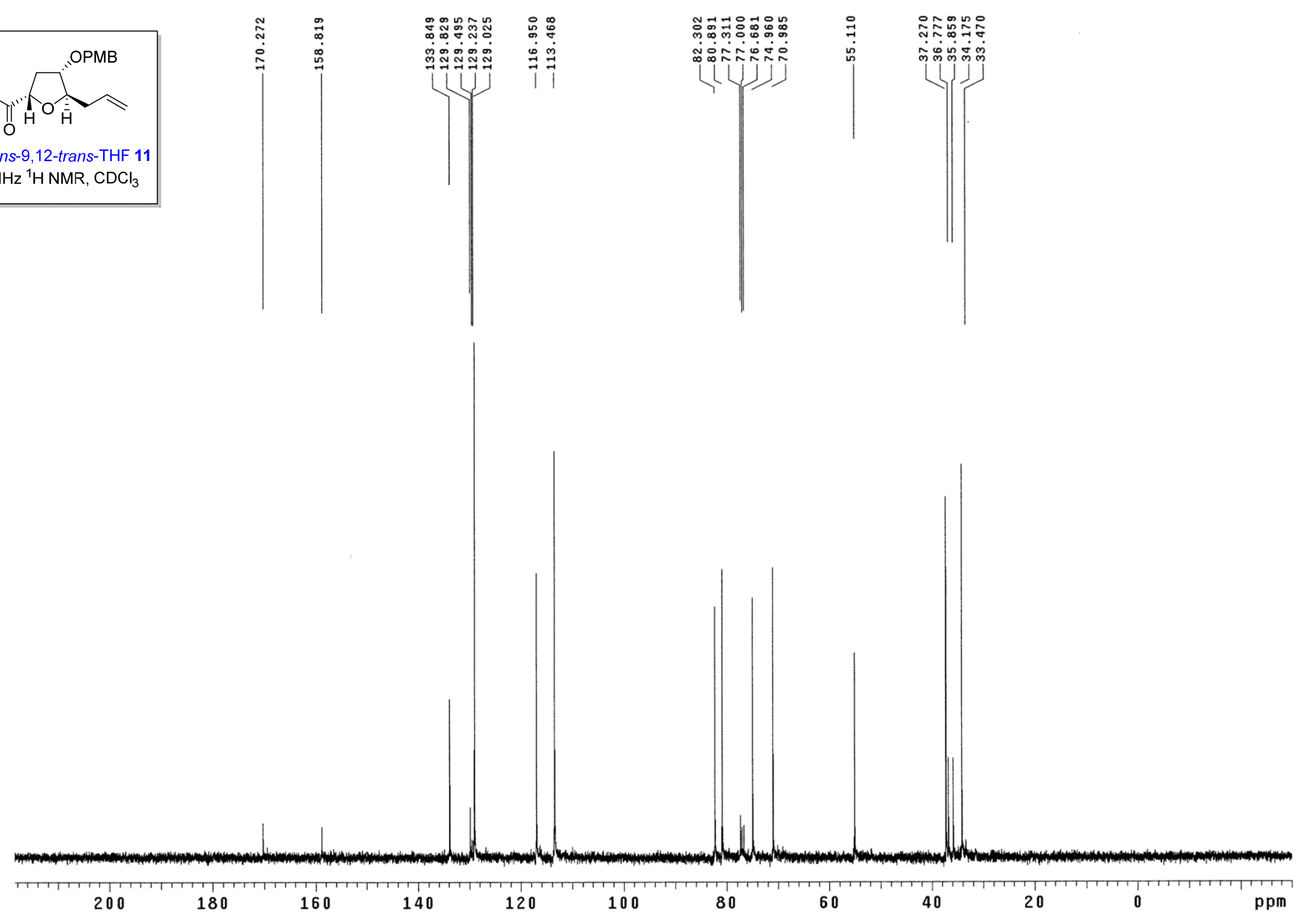


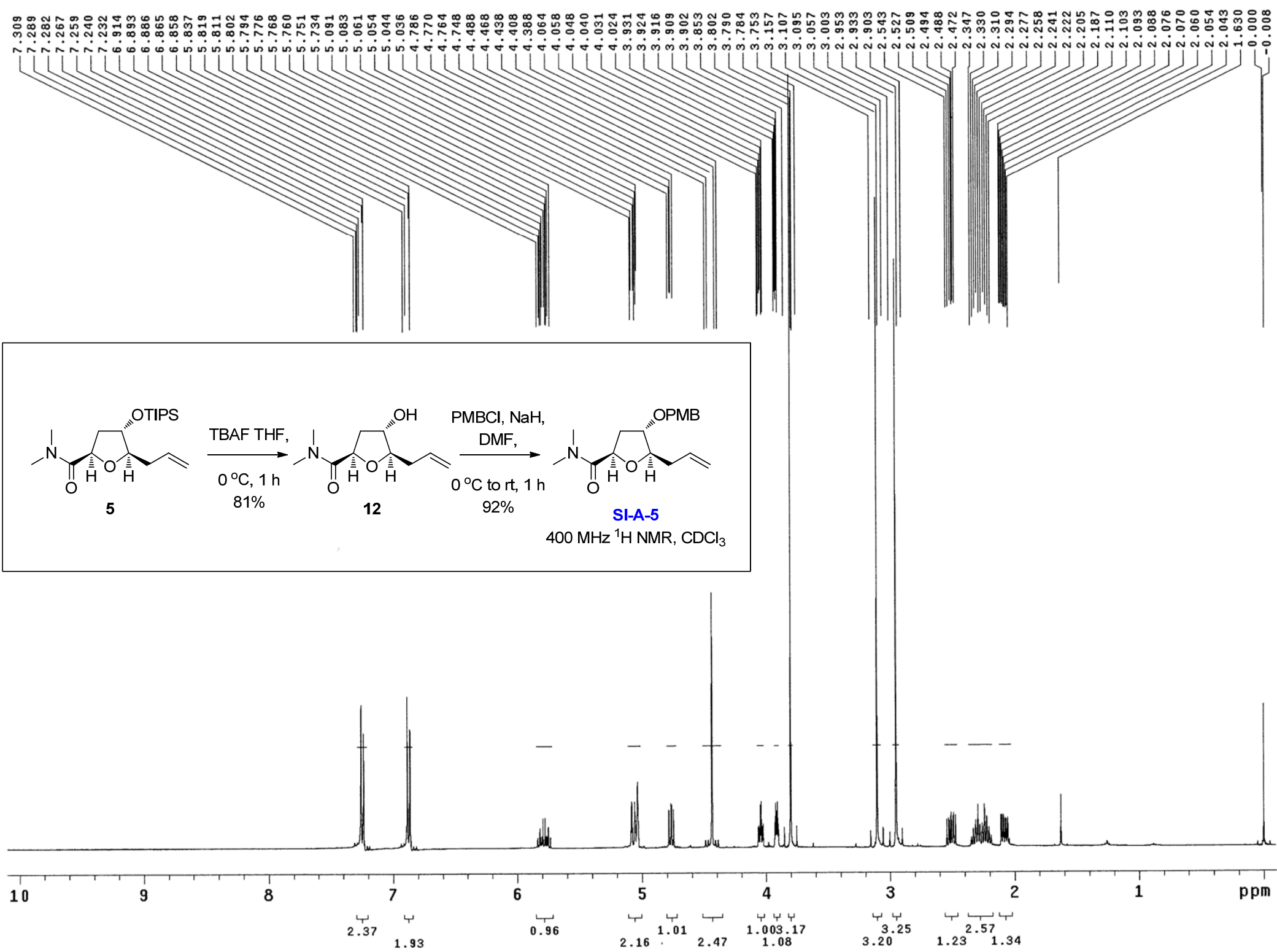




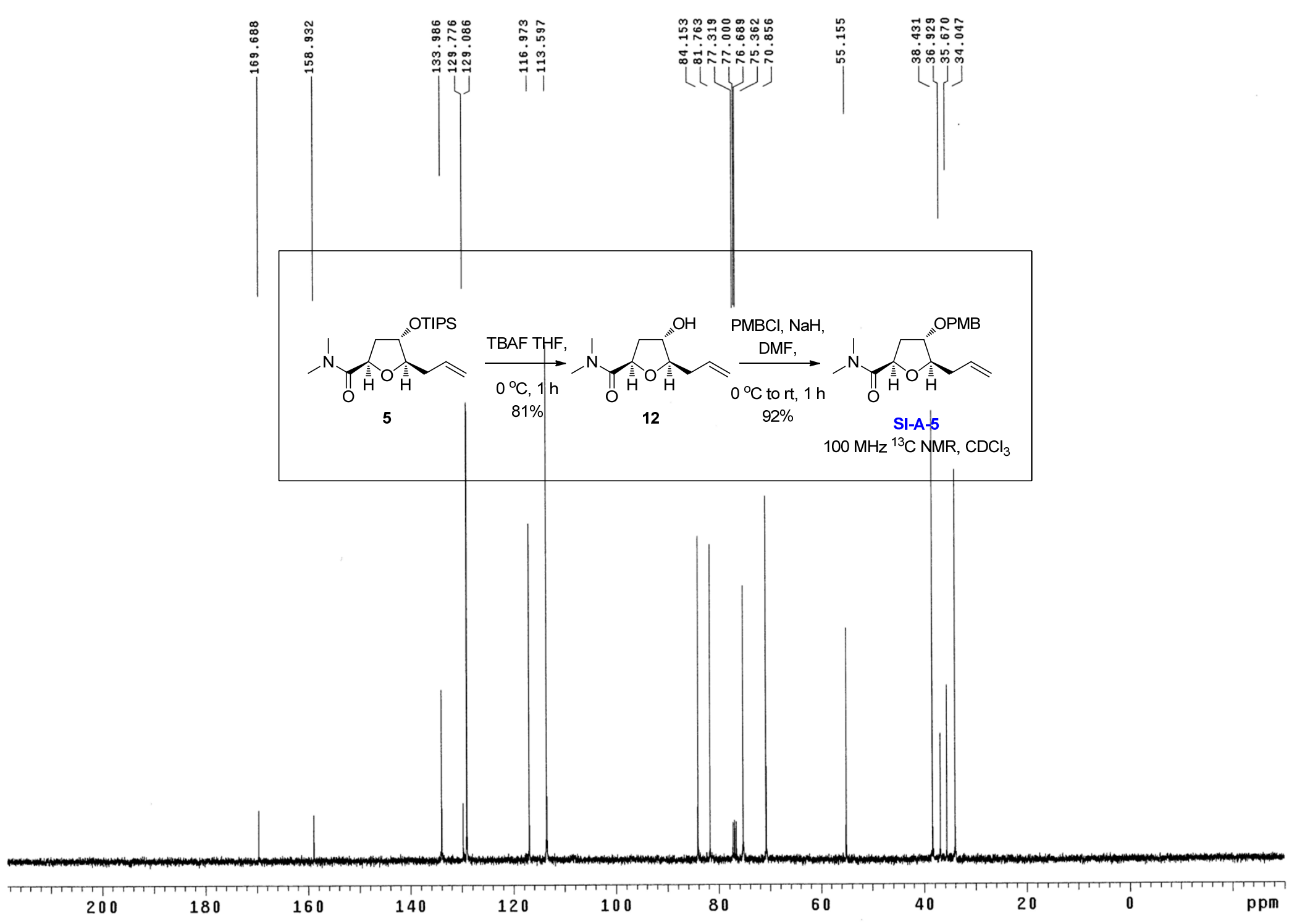



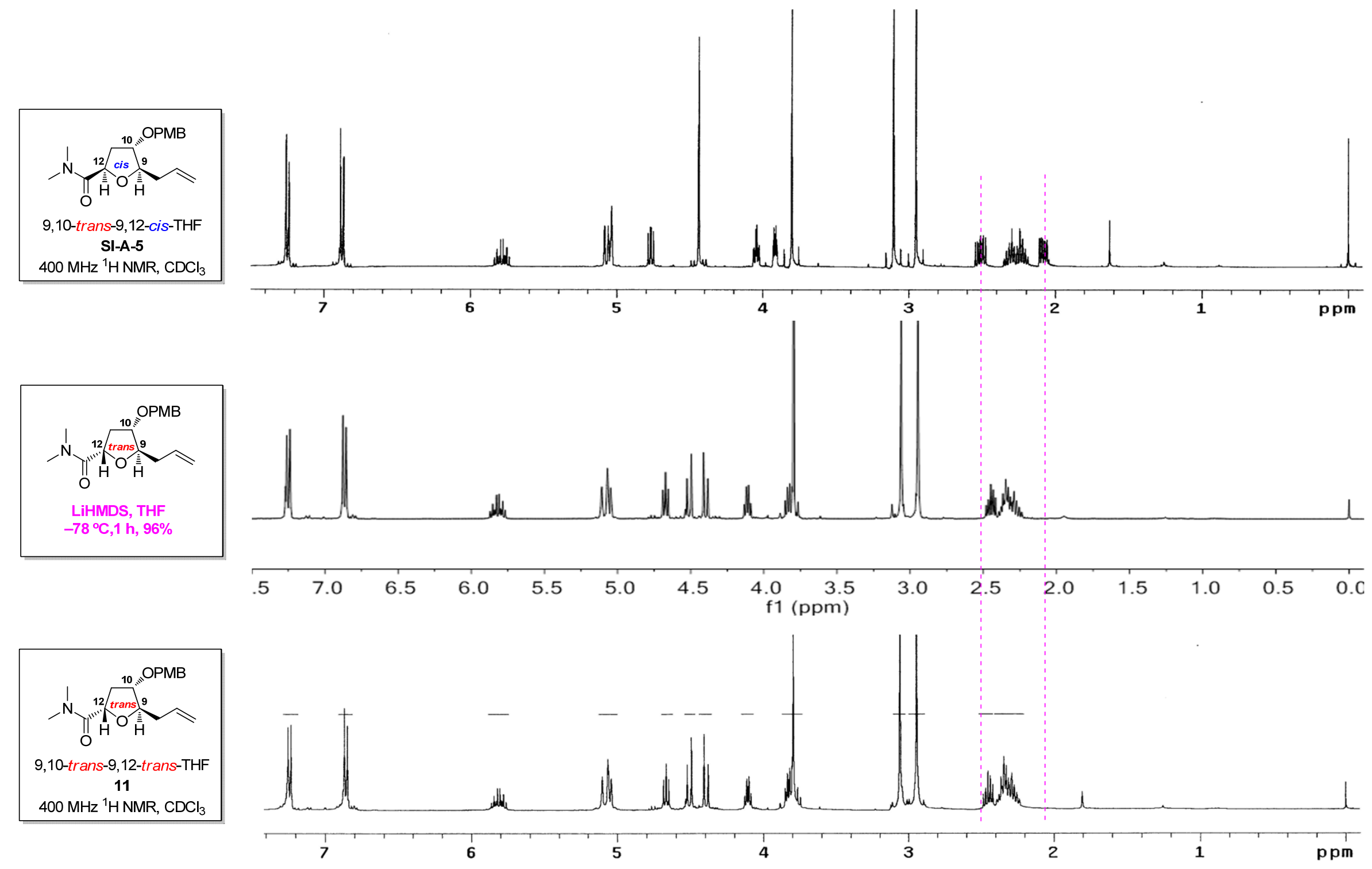


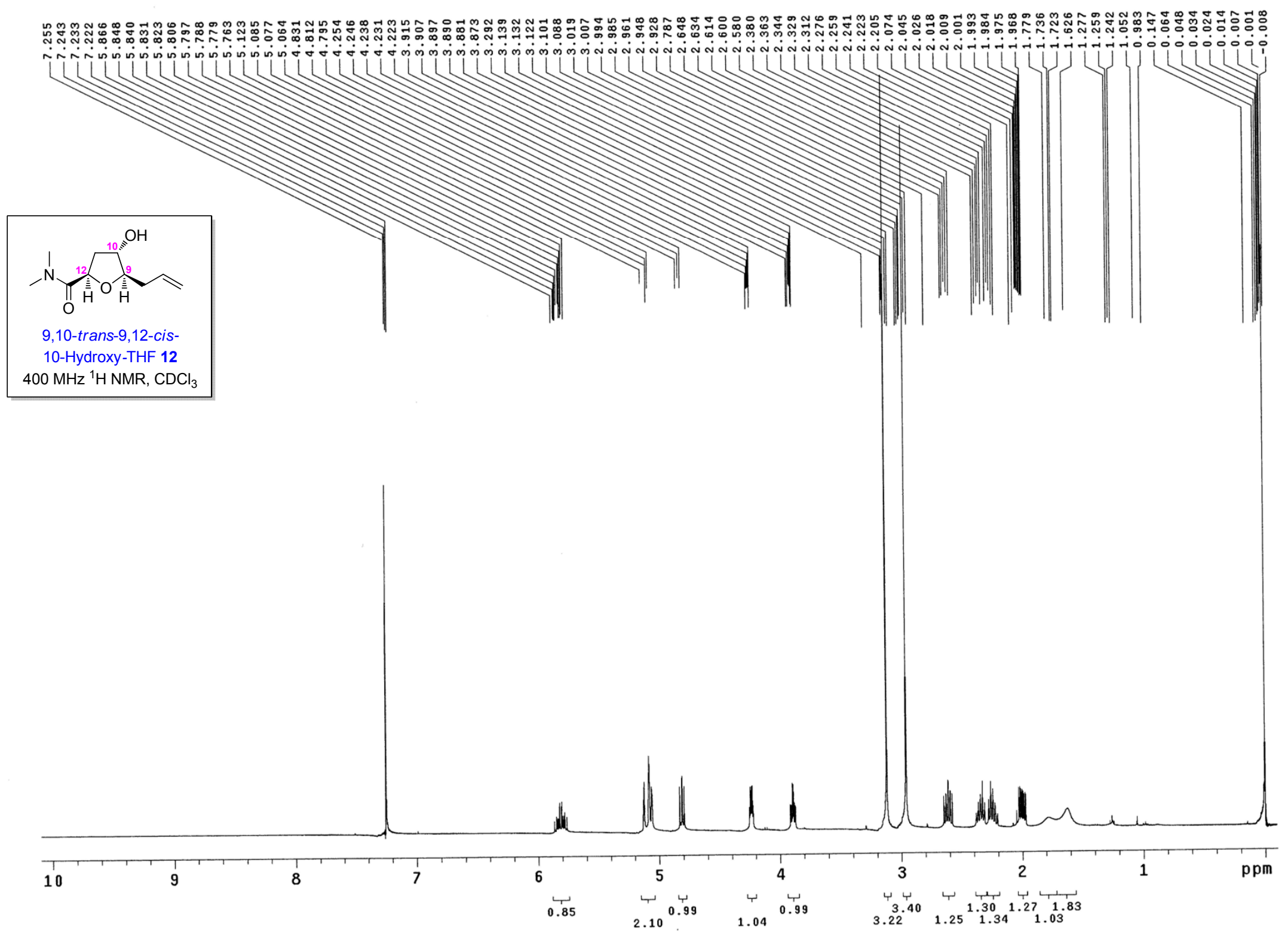




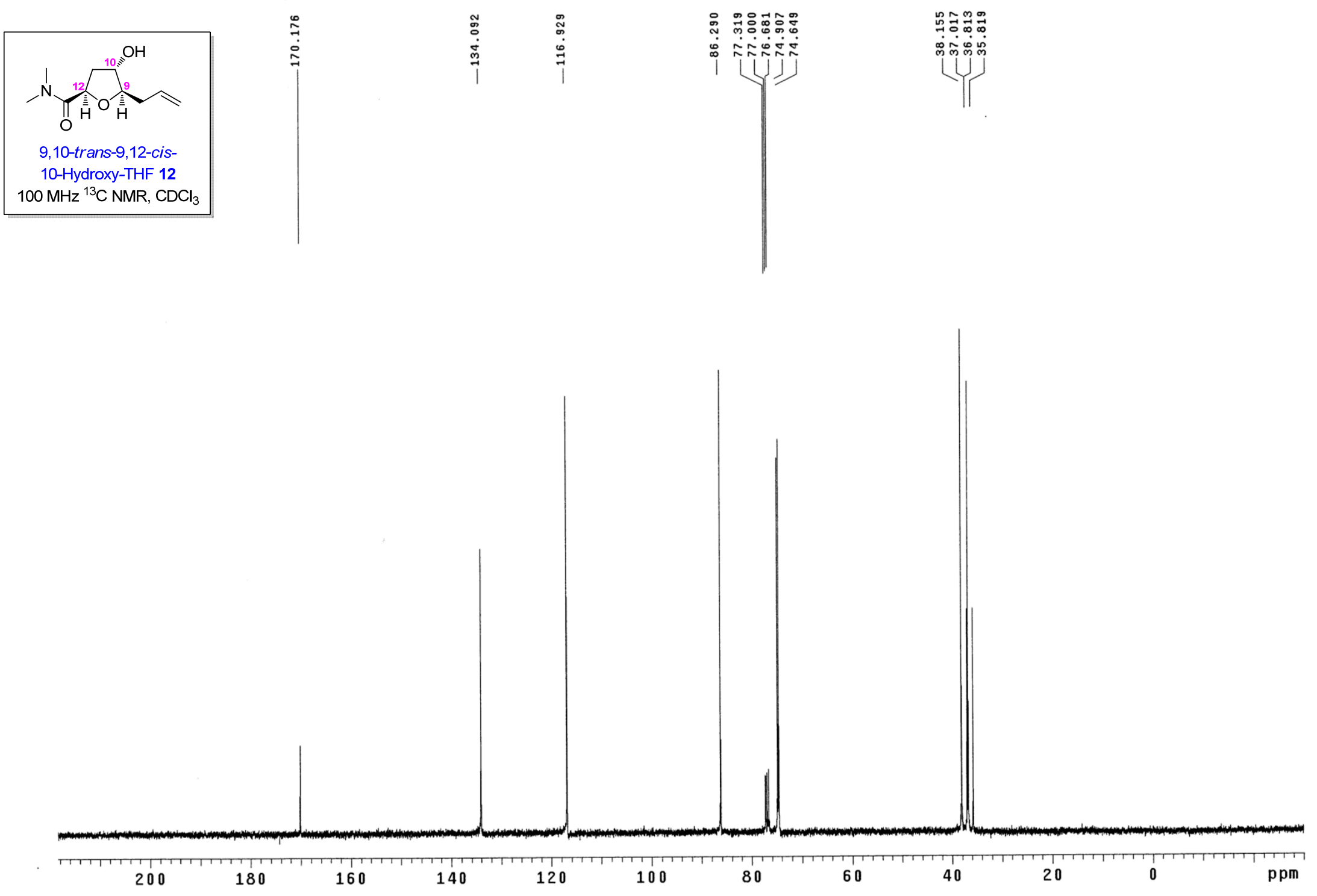




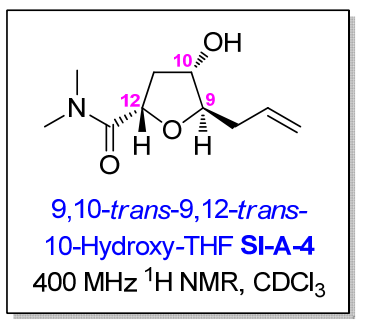

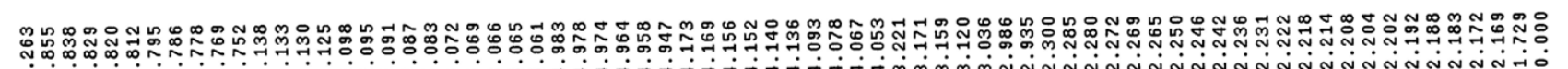

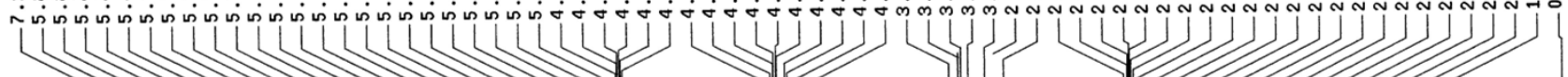

$400 \mathrm{MHz}^{1} \mathrm{H} \mathrm{NMR} \mathrm{CDCl}_{3}$

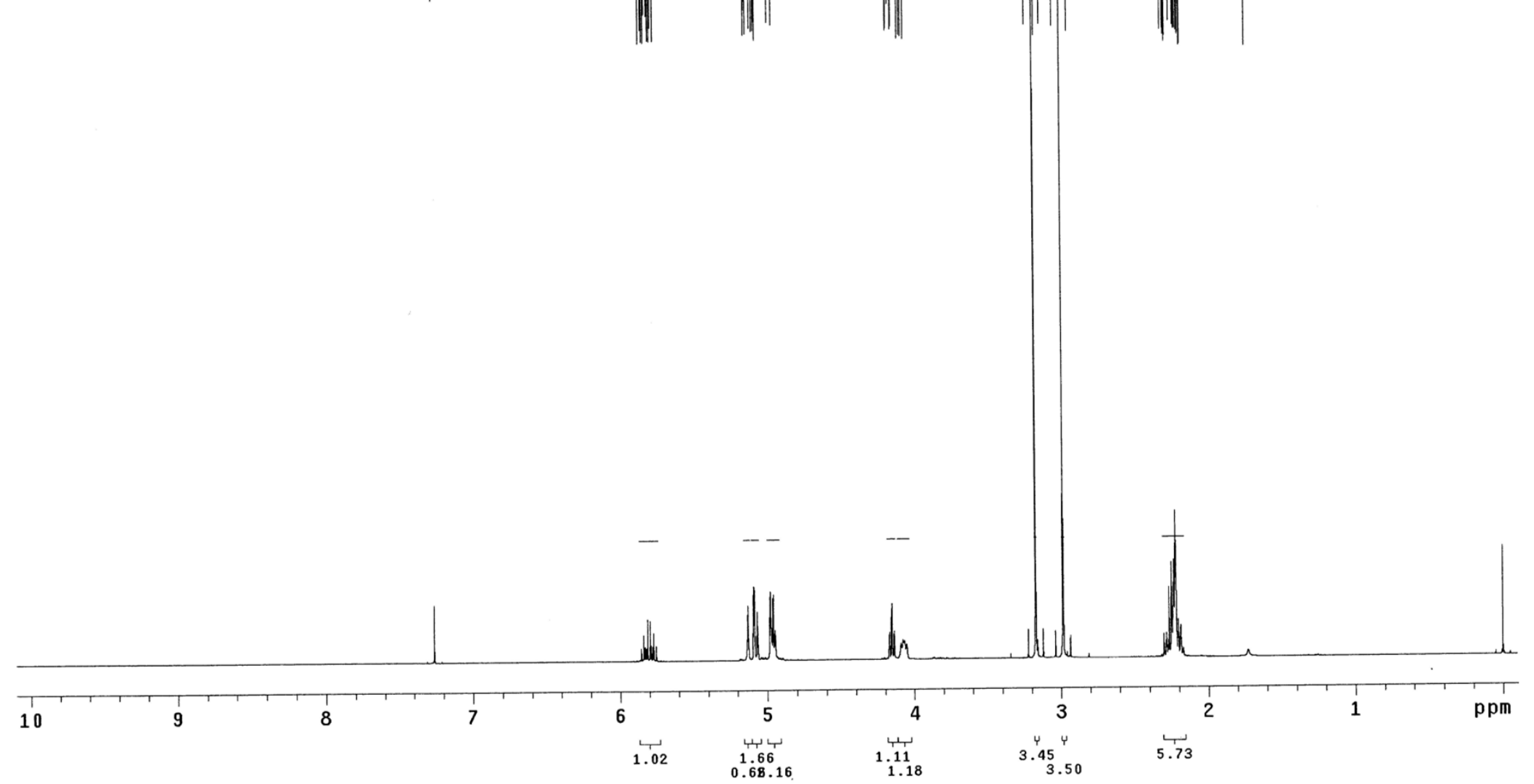



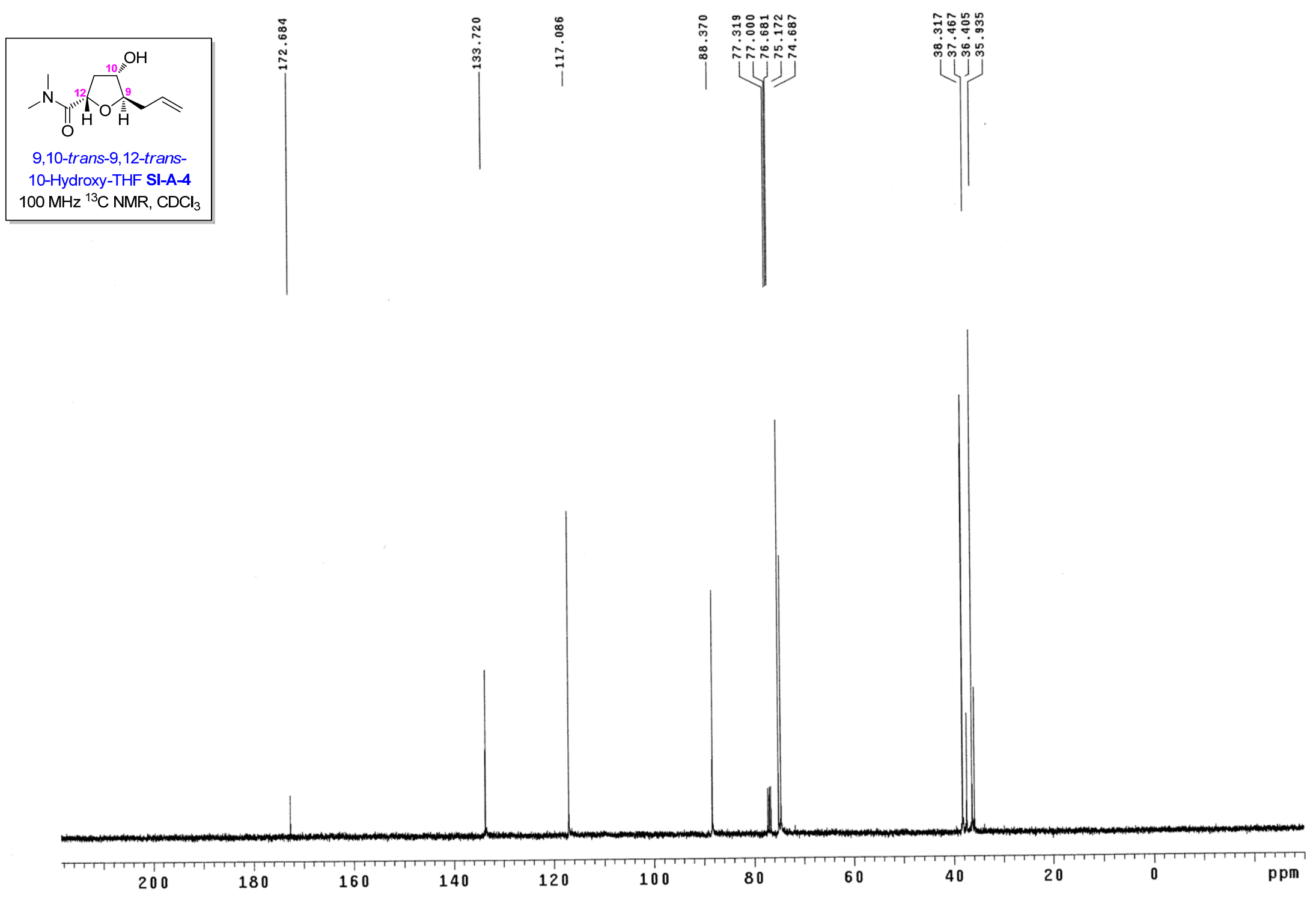


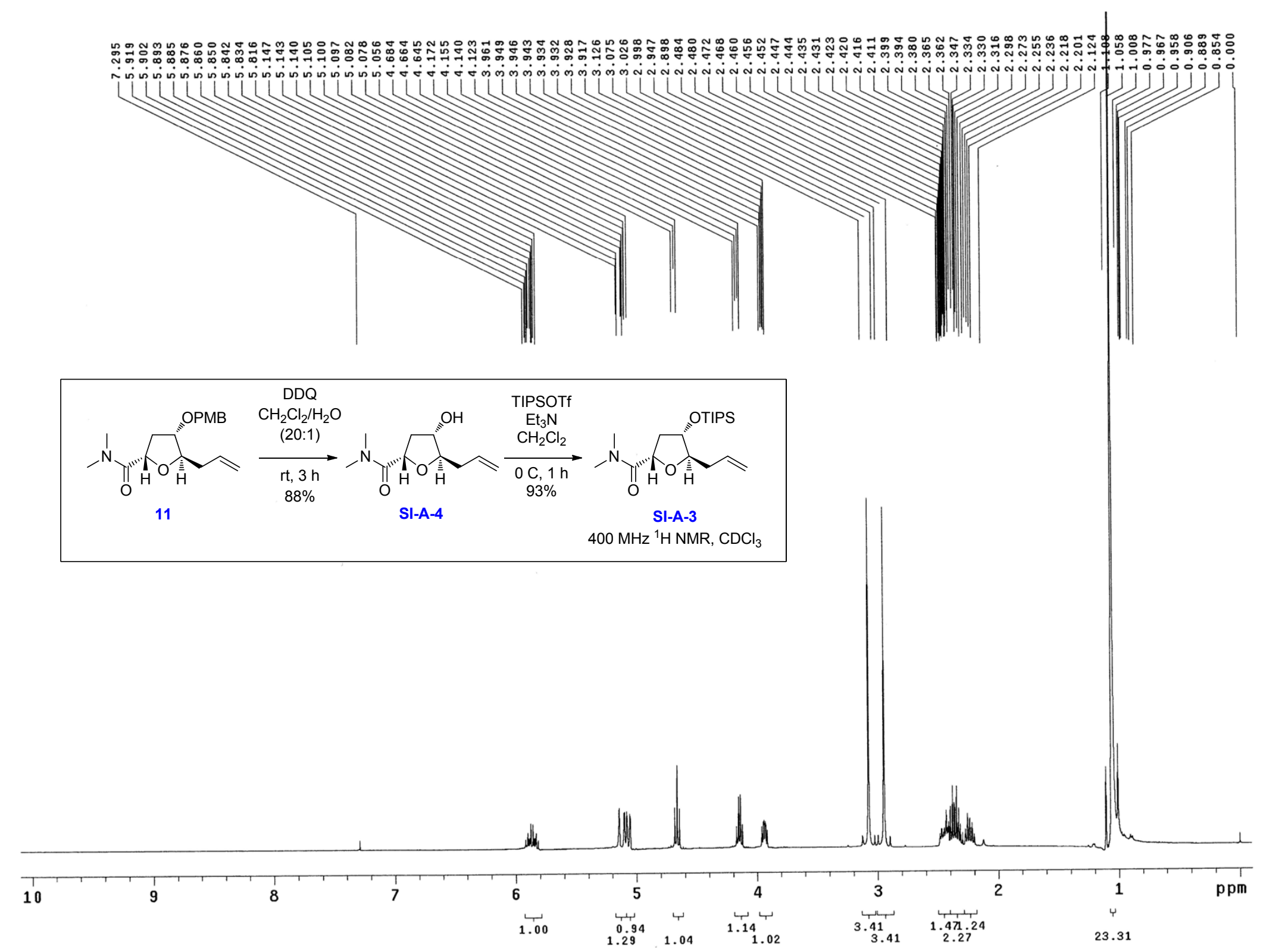



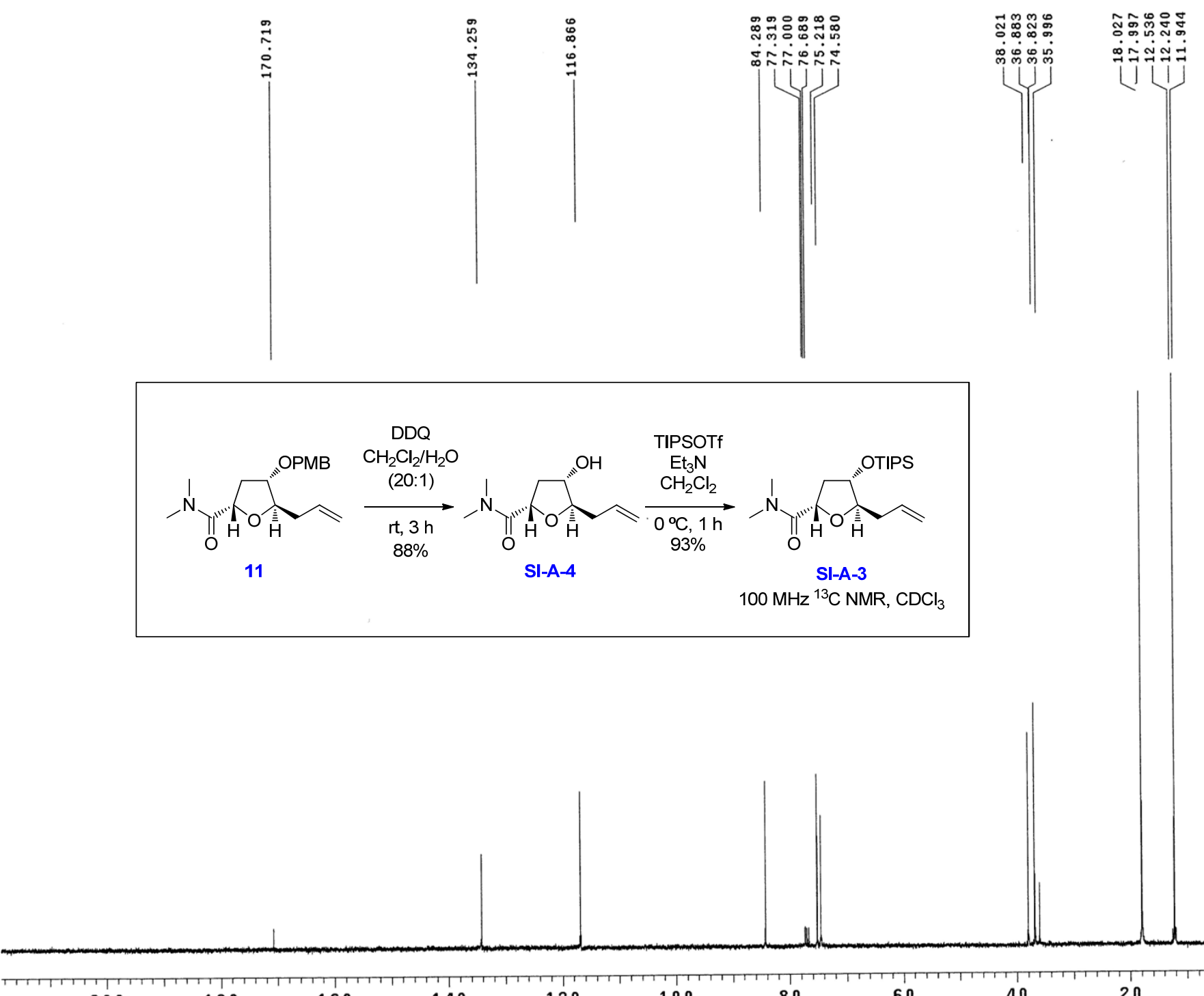


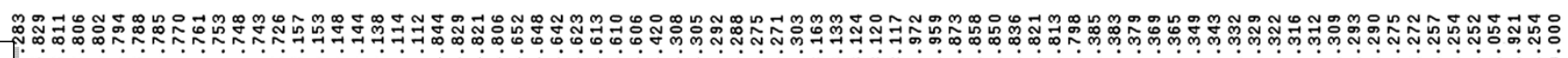
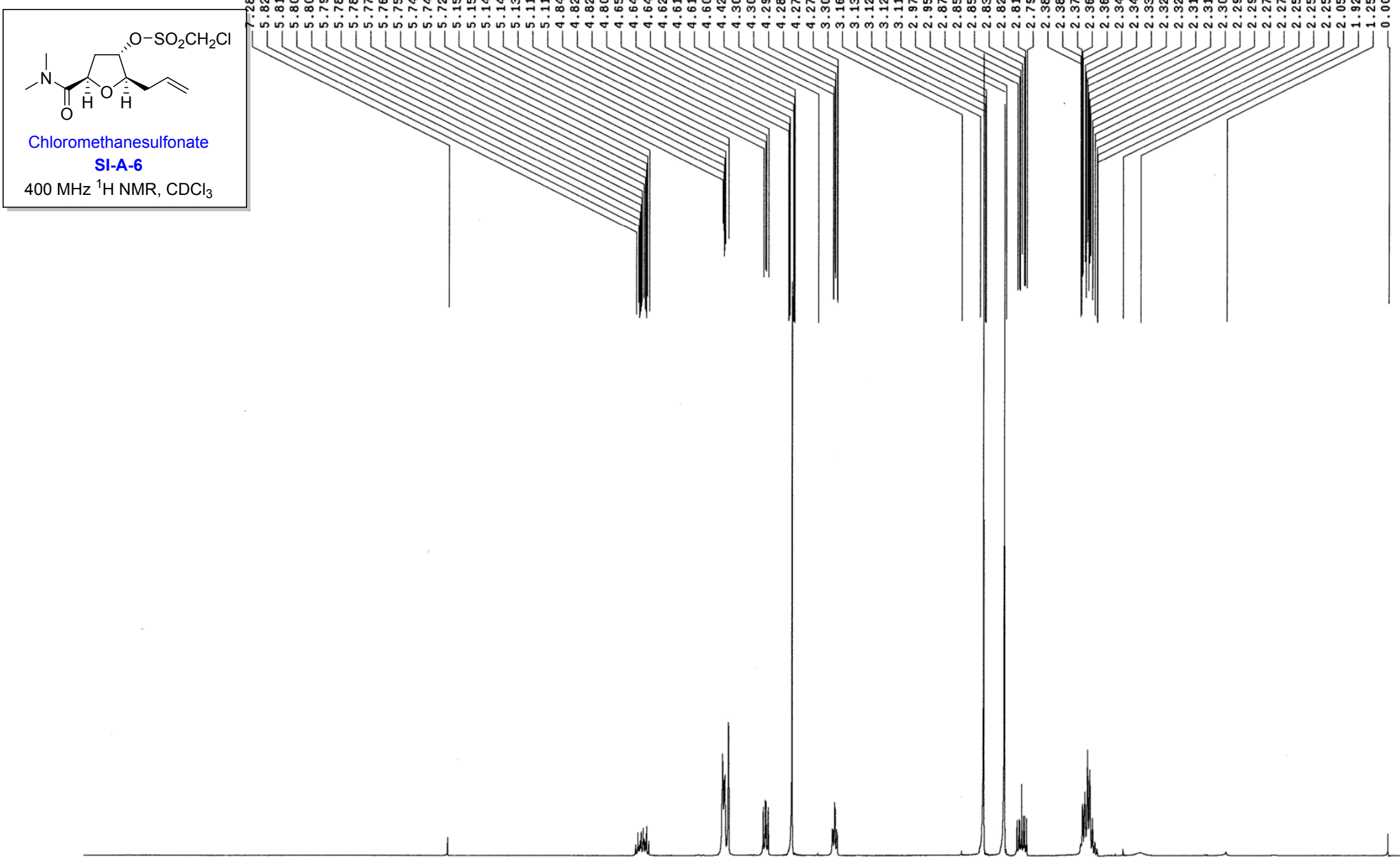

10

8

6

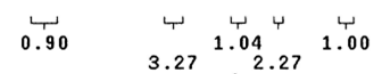

$\underset{3.68}{\sqrt[4]{3.68}}$ 


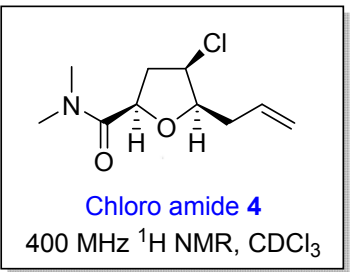

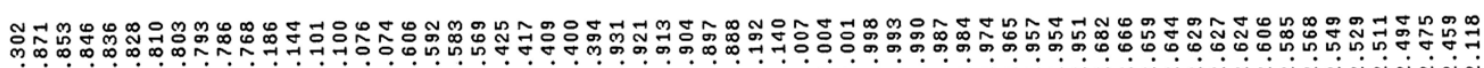

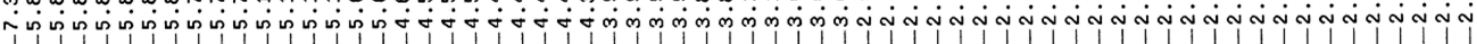

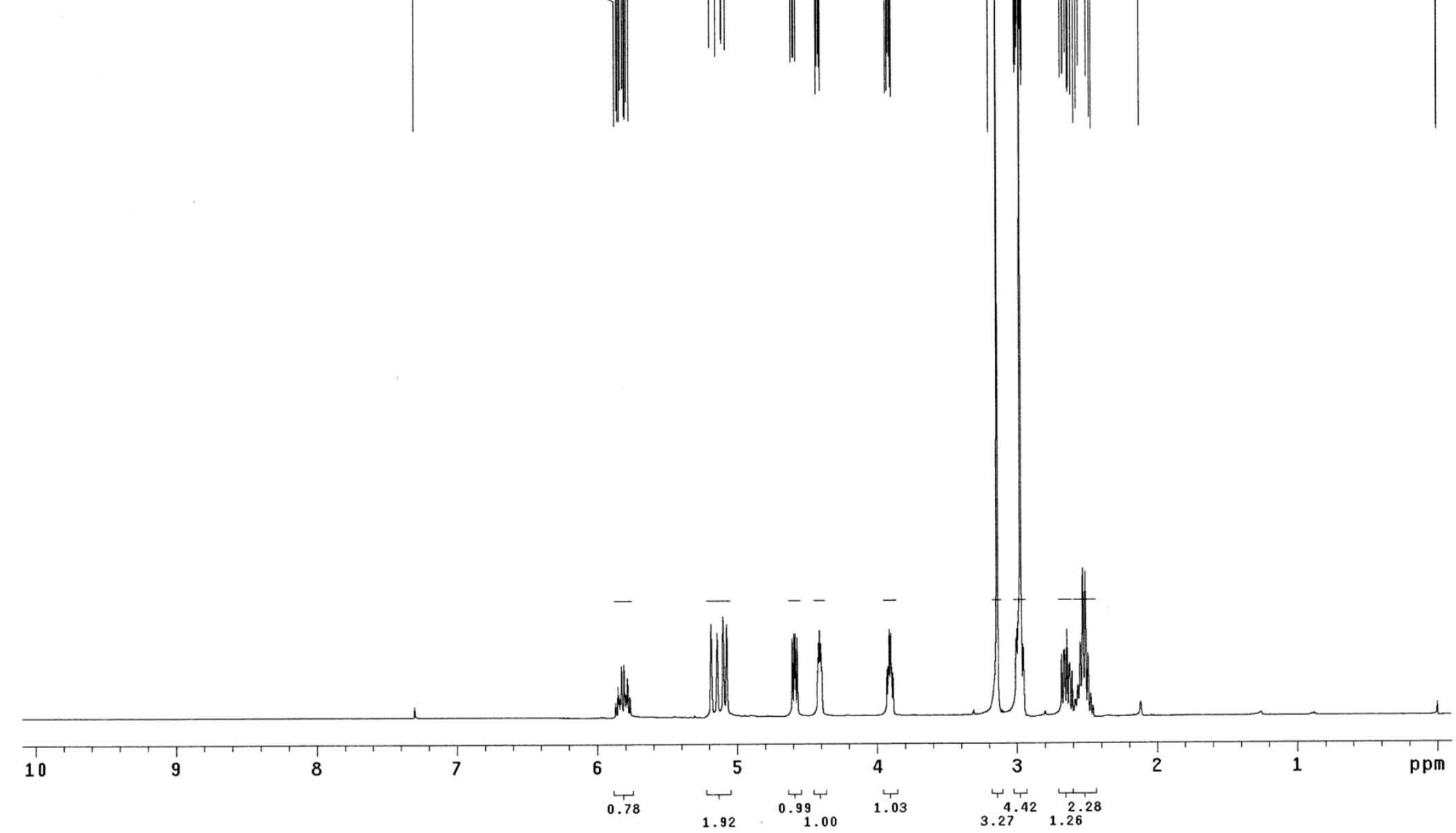




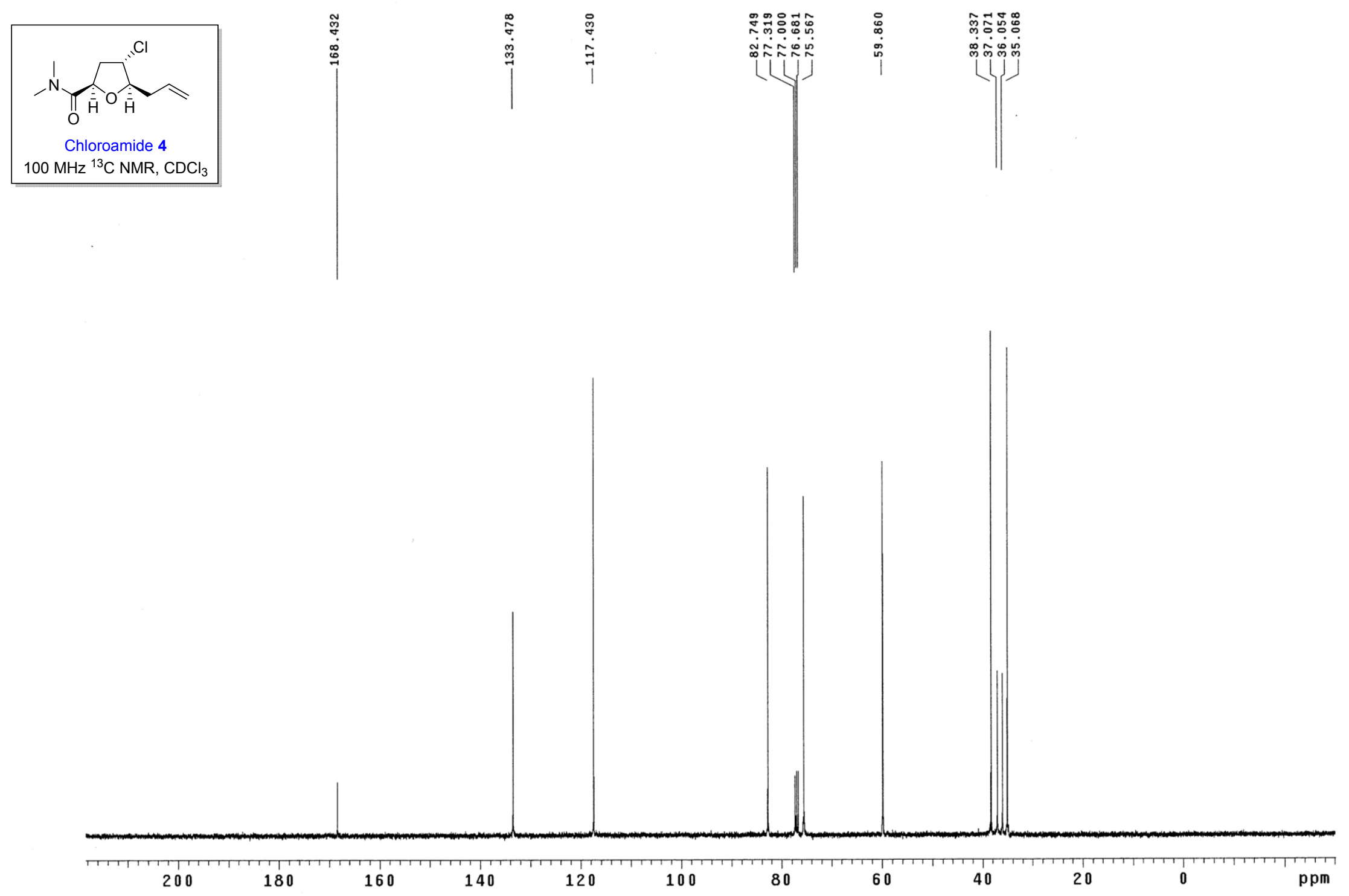




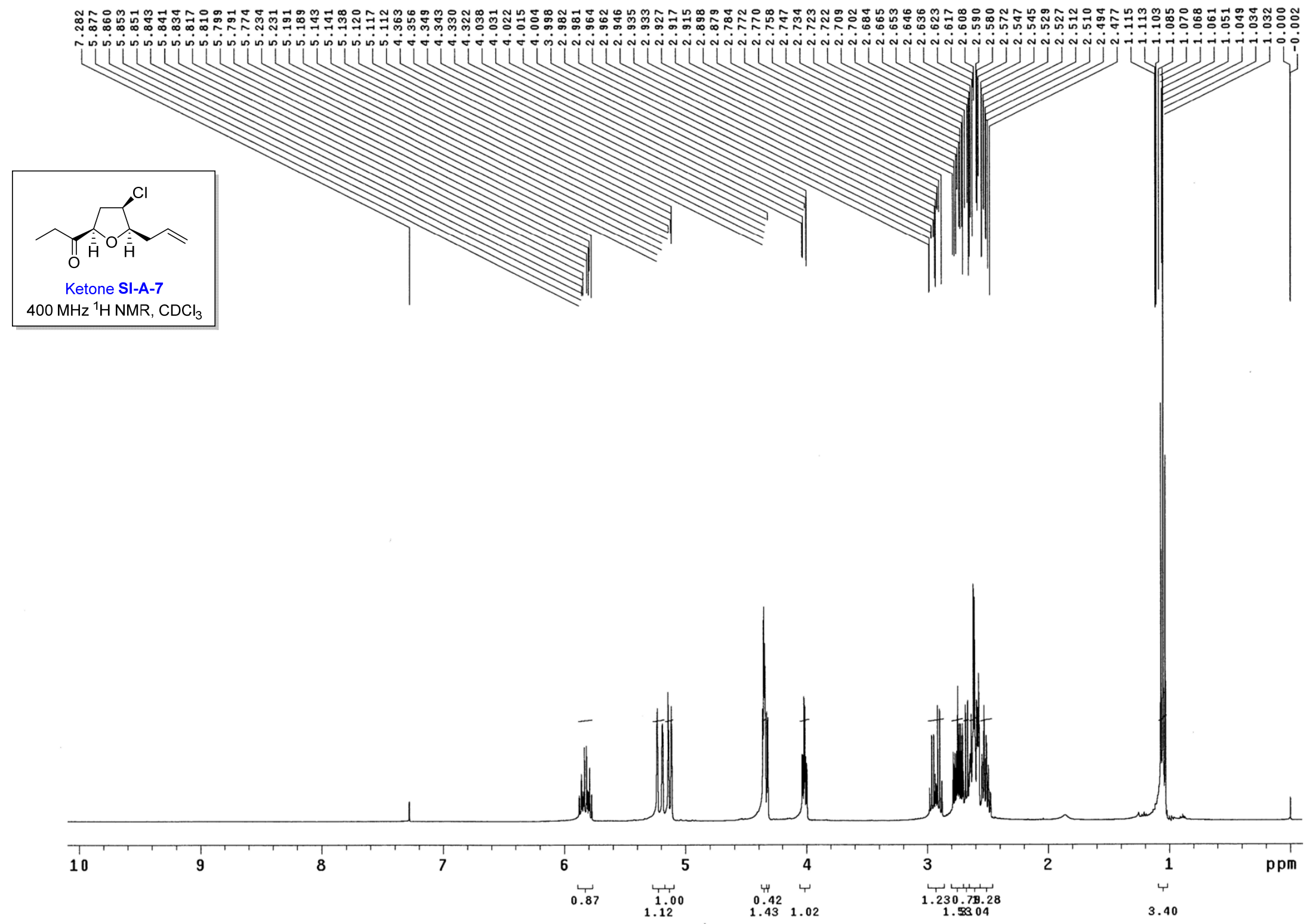




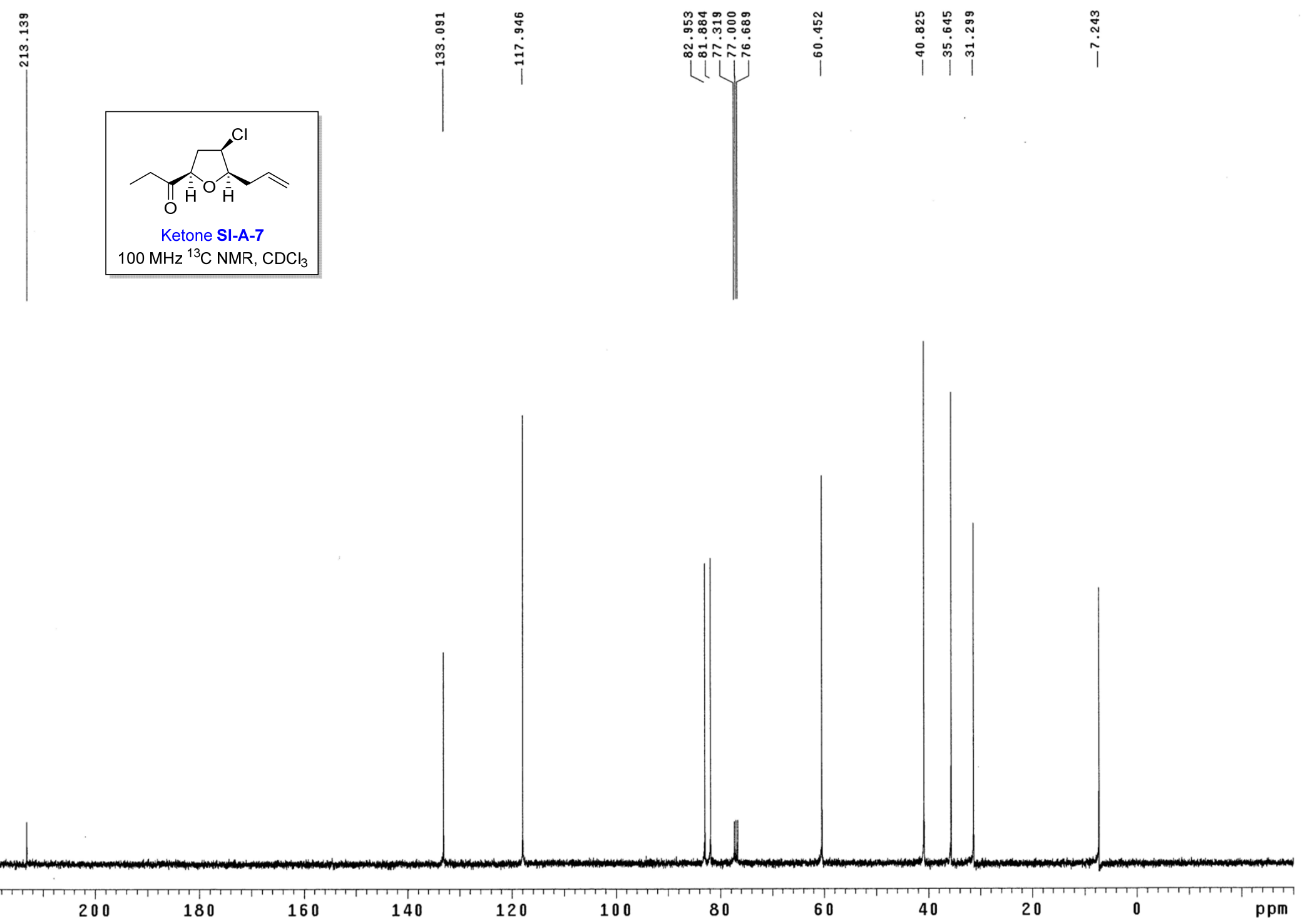




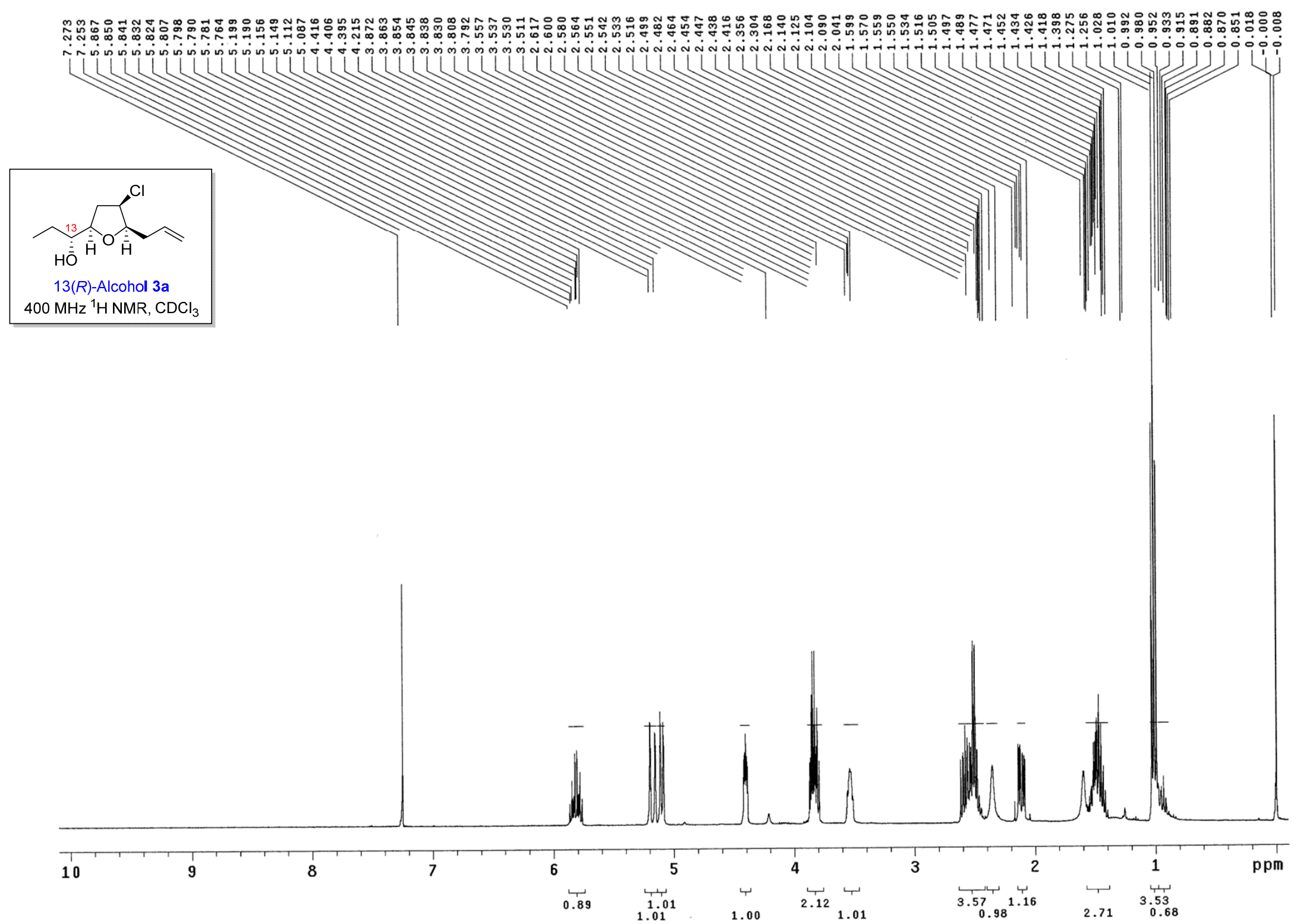



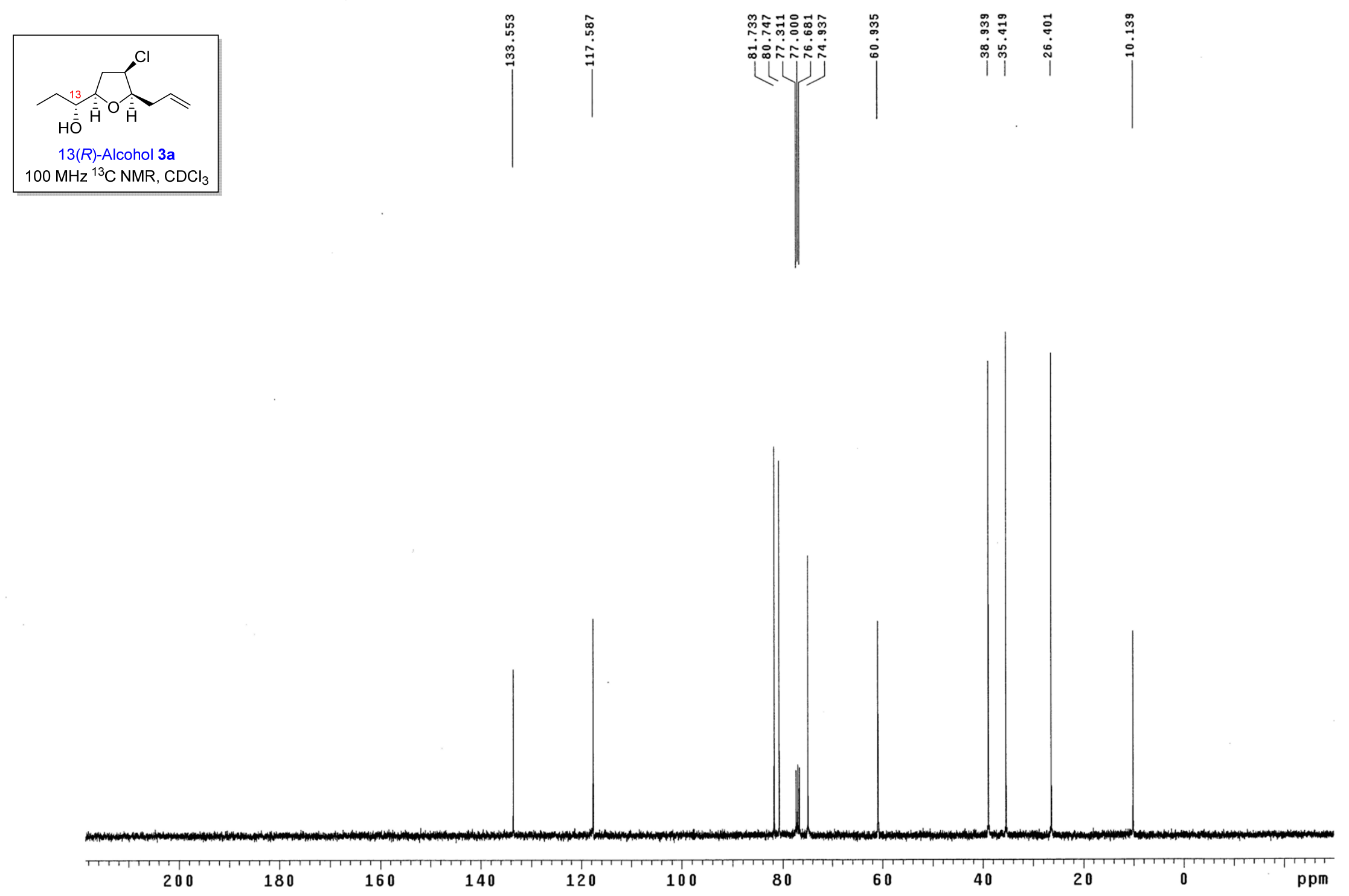


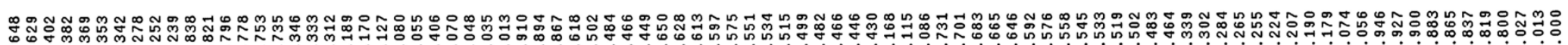

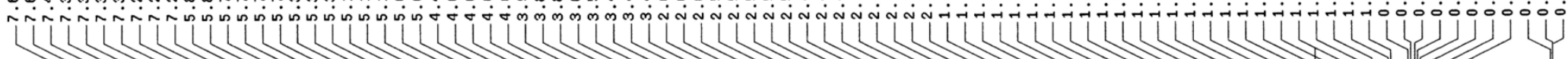

(R)-Mosher Ester SI-A-8
400 MHz ${ }^{1} \mathrm{H}$ NMR, CDCl
OMe
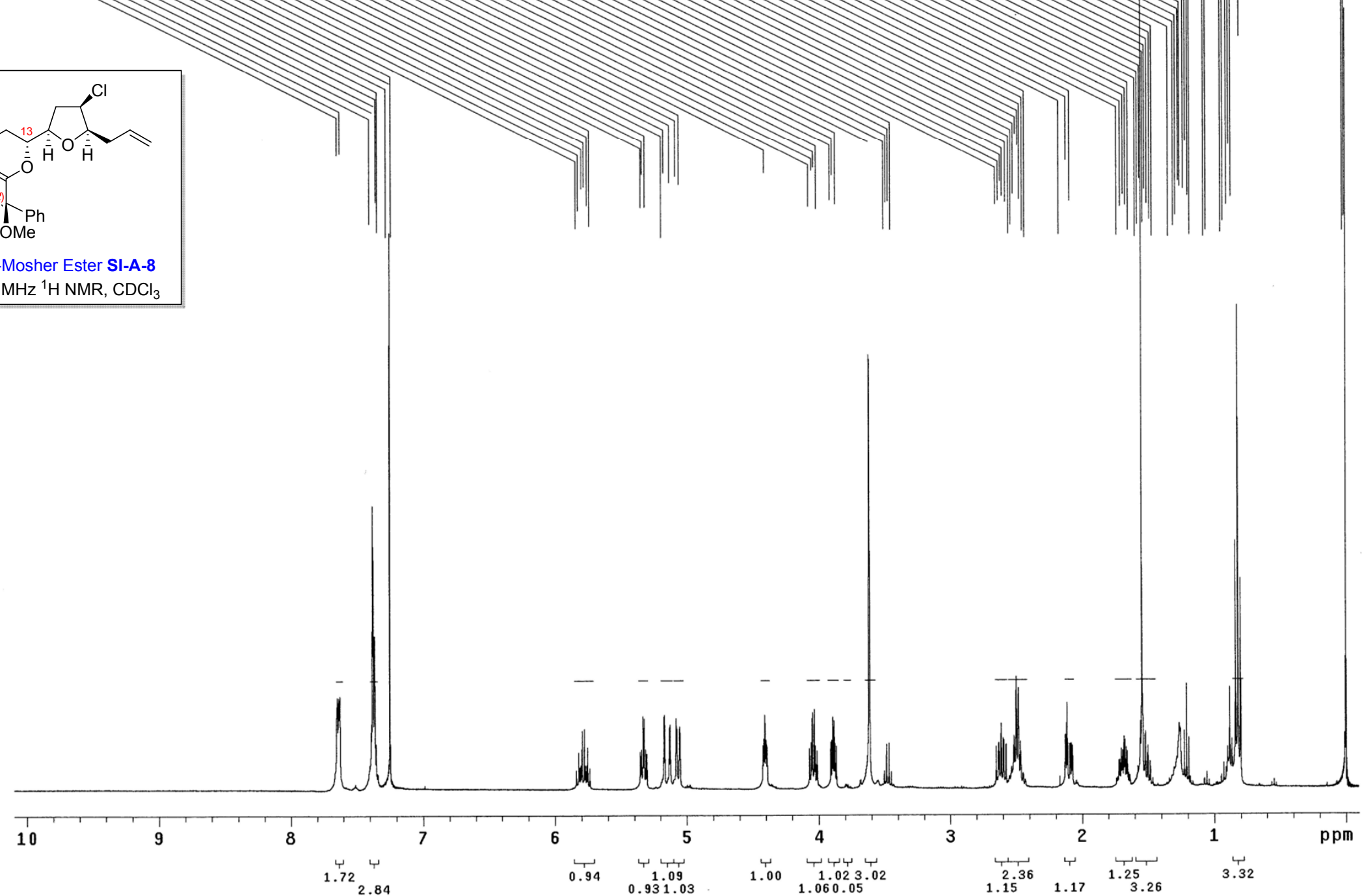


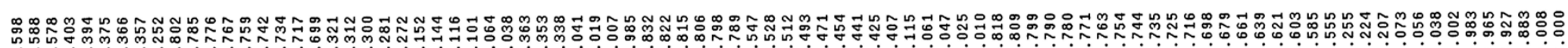
- ن-
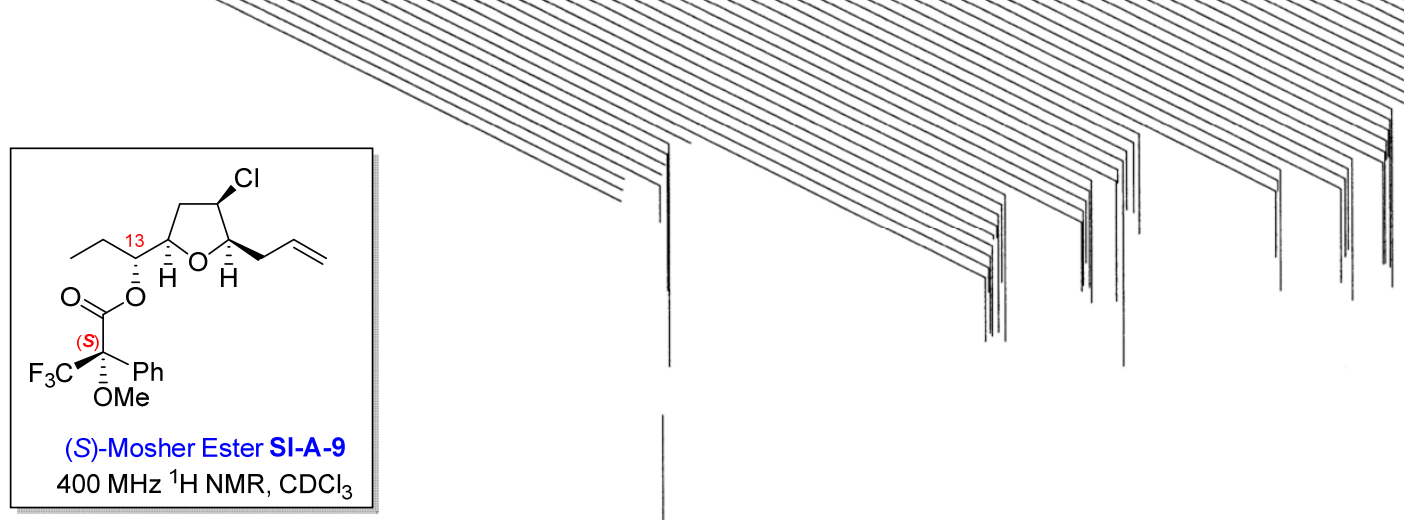

$$
\mathrm{MHz}^{1} \mathrm{HNMR}, \mathrm{CDCl}_{3}
$$

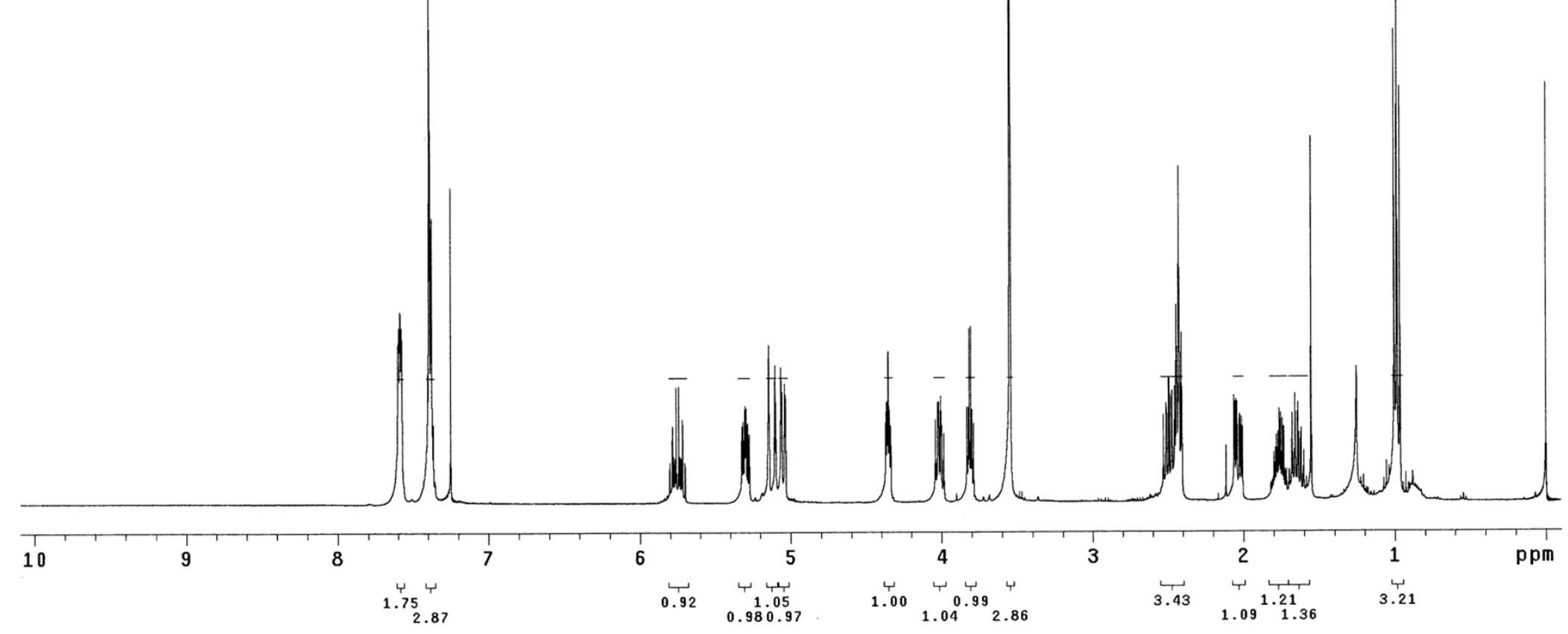




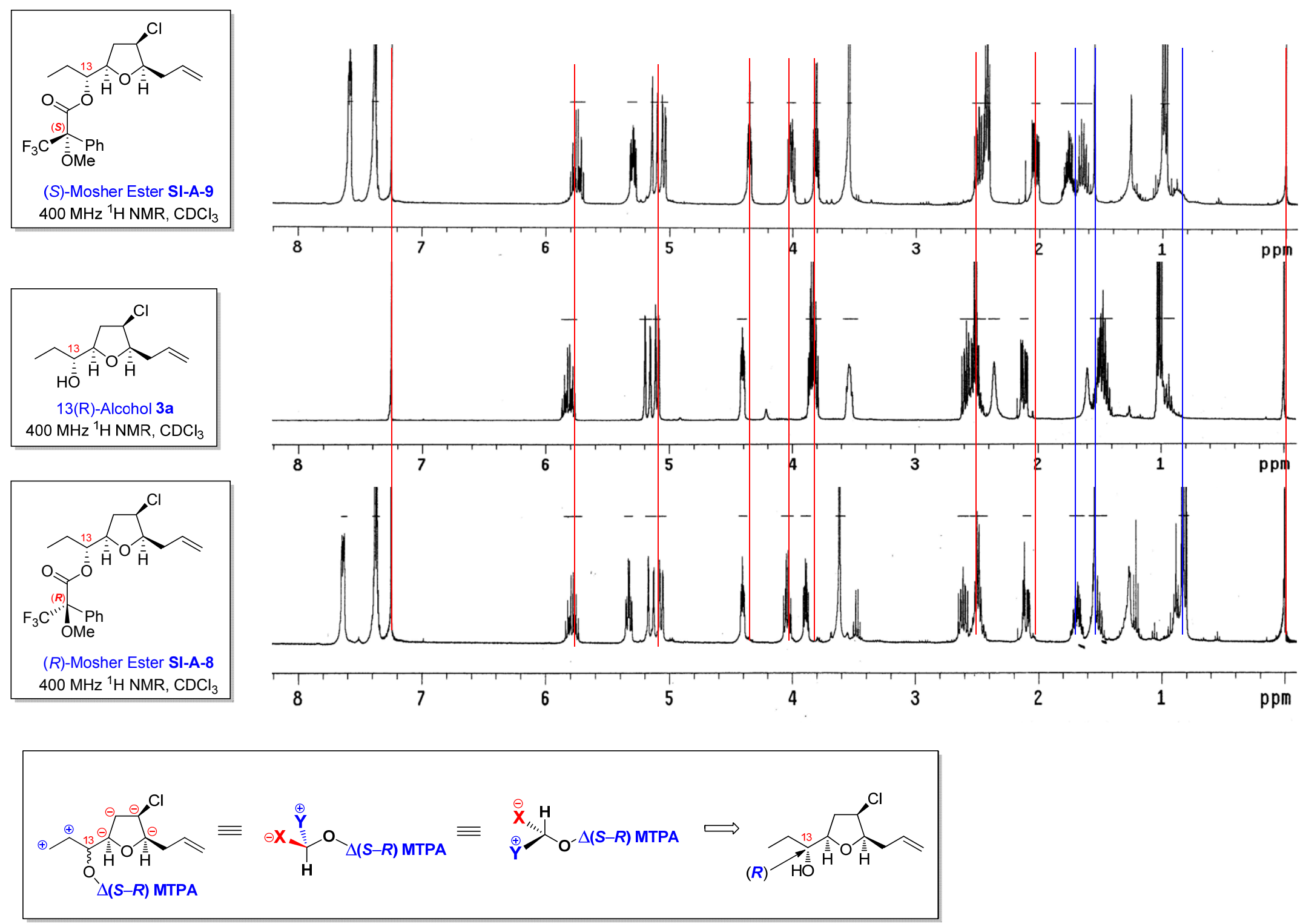




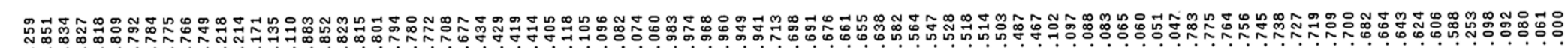

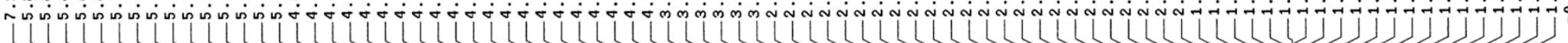
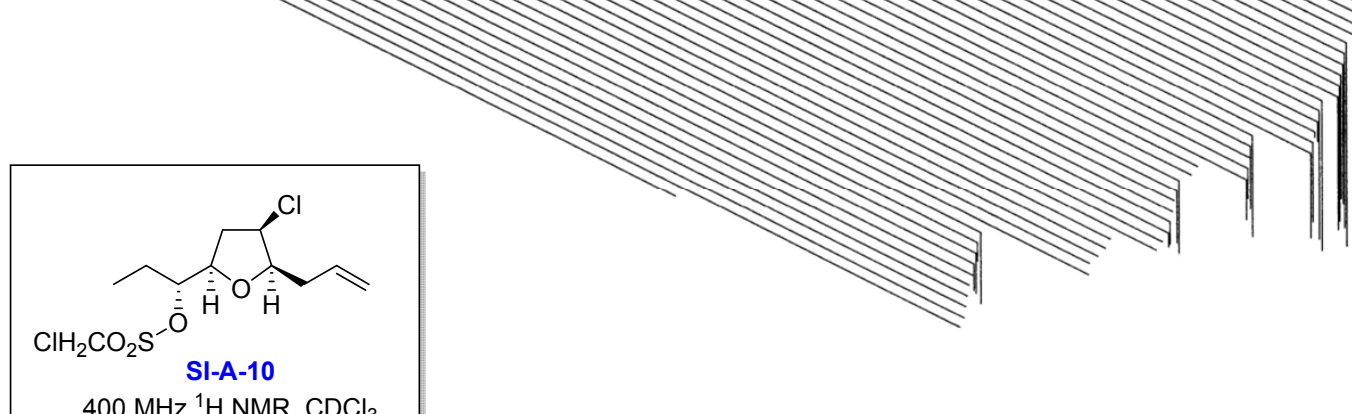

$400 \mathrm{MHz}{ }^{1} \mathrm{H}$ NMR, $\mathrm{CDCl}$

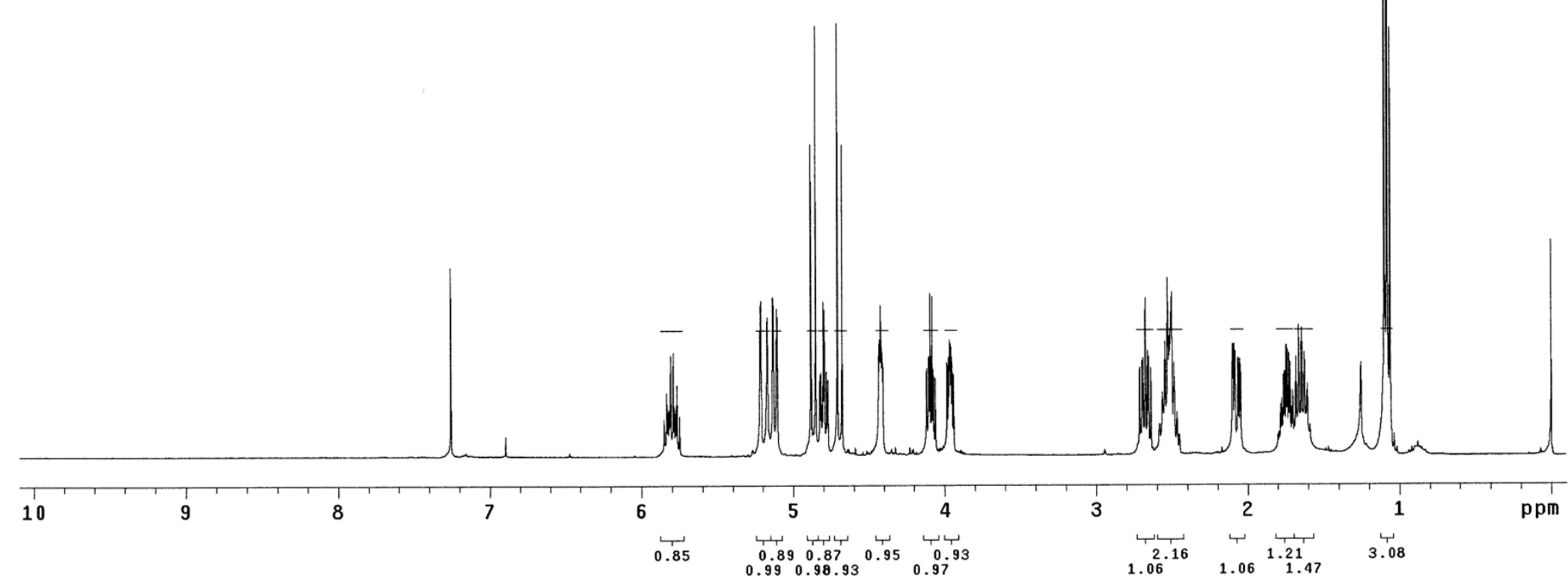




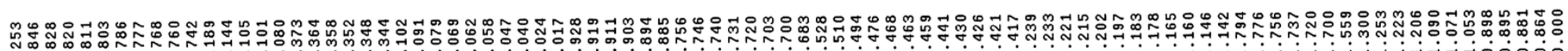

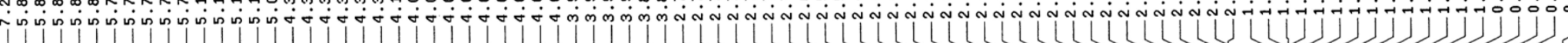

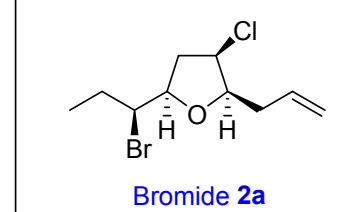

$400 \mathrm{MHz}^{1} \mathrm{H} \mathrm{NMR}, \mathrm{CDCl}_{3}$
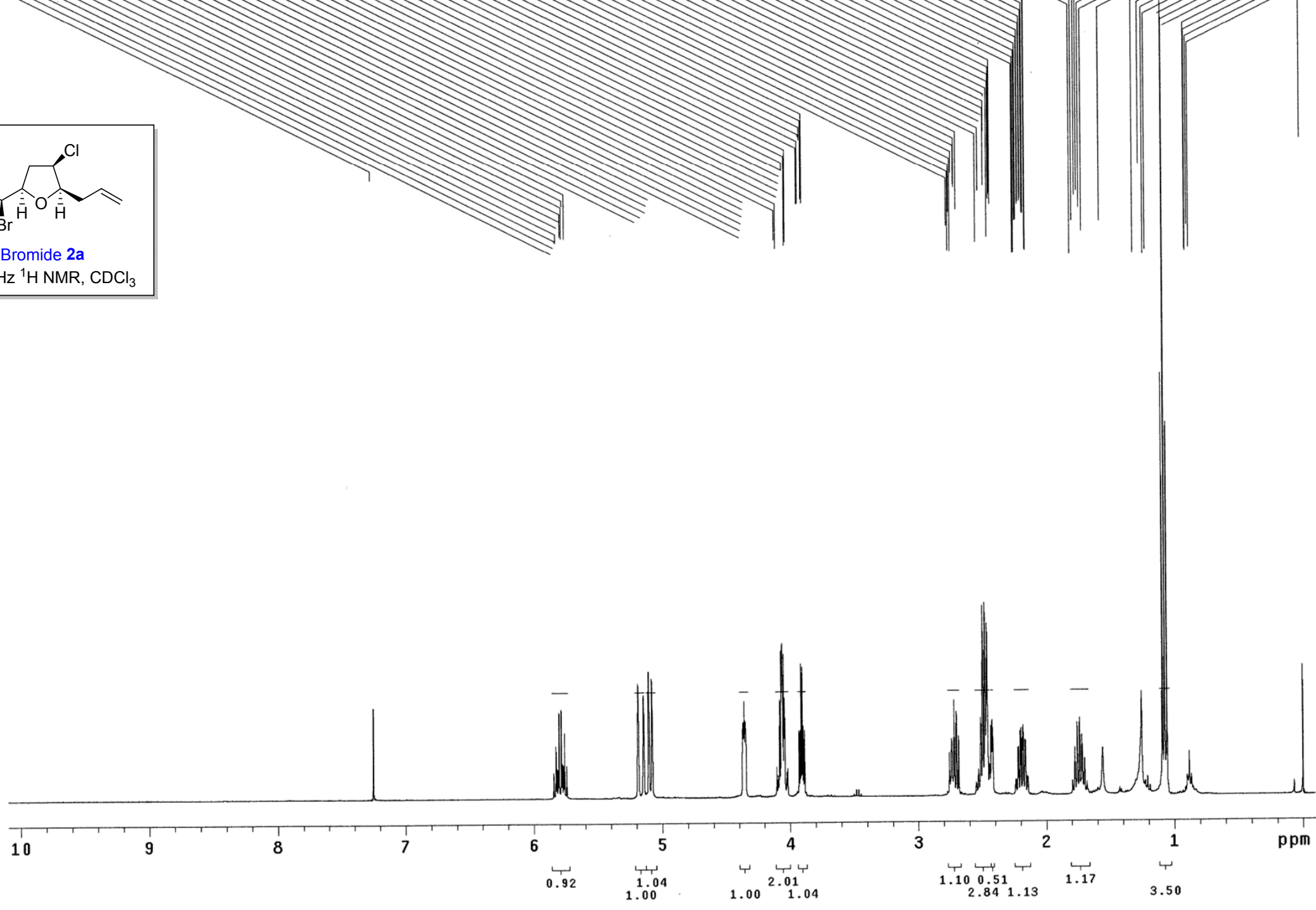

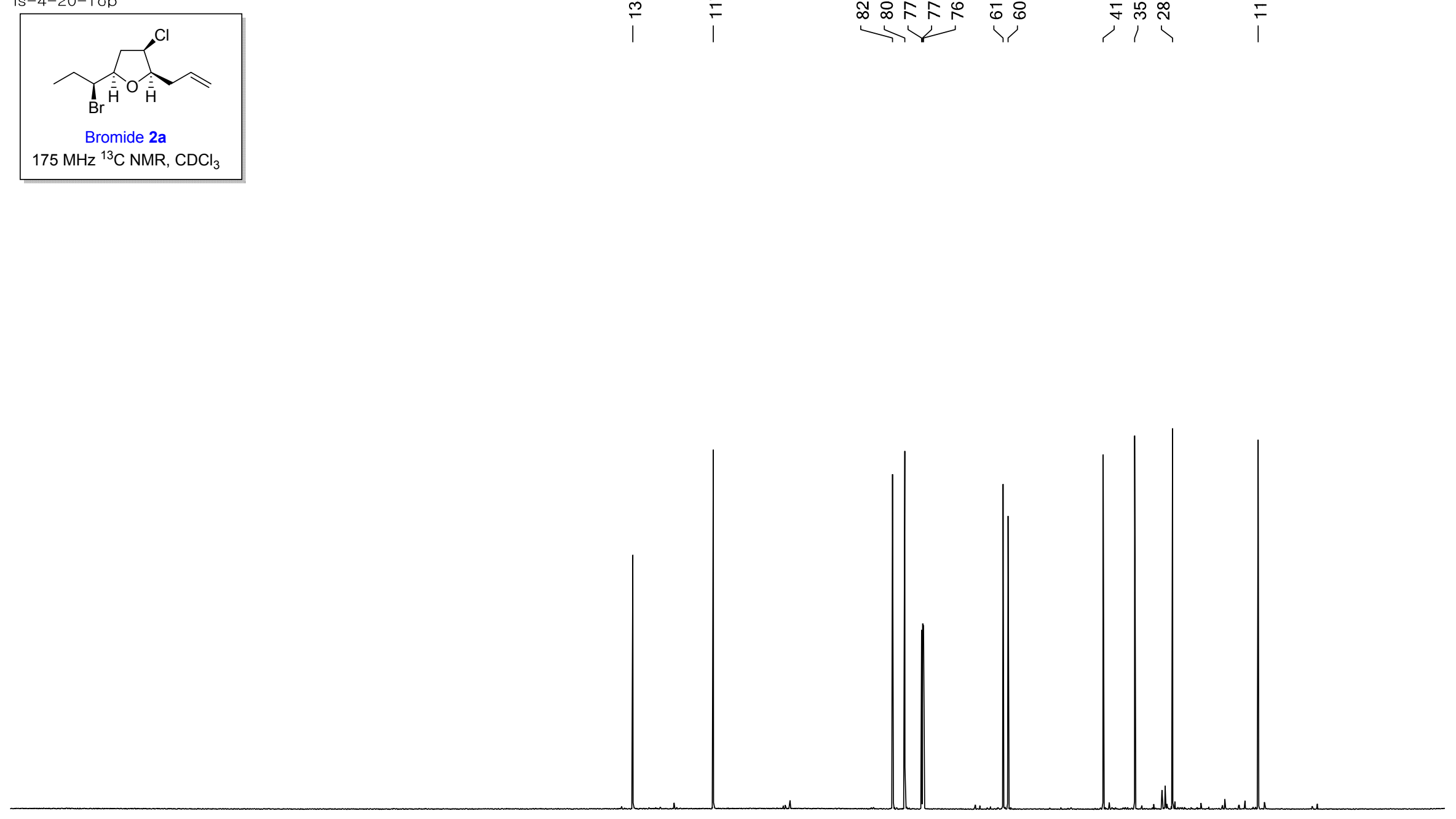

$25024023022021020019018017016015014013012011010090 \quad 8070 \quad 6050 \quad 4030 \quad 2010 \quad 0 \quad-10-20$ f1 (ppm) 

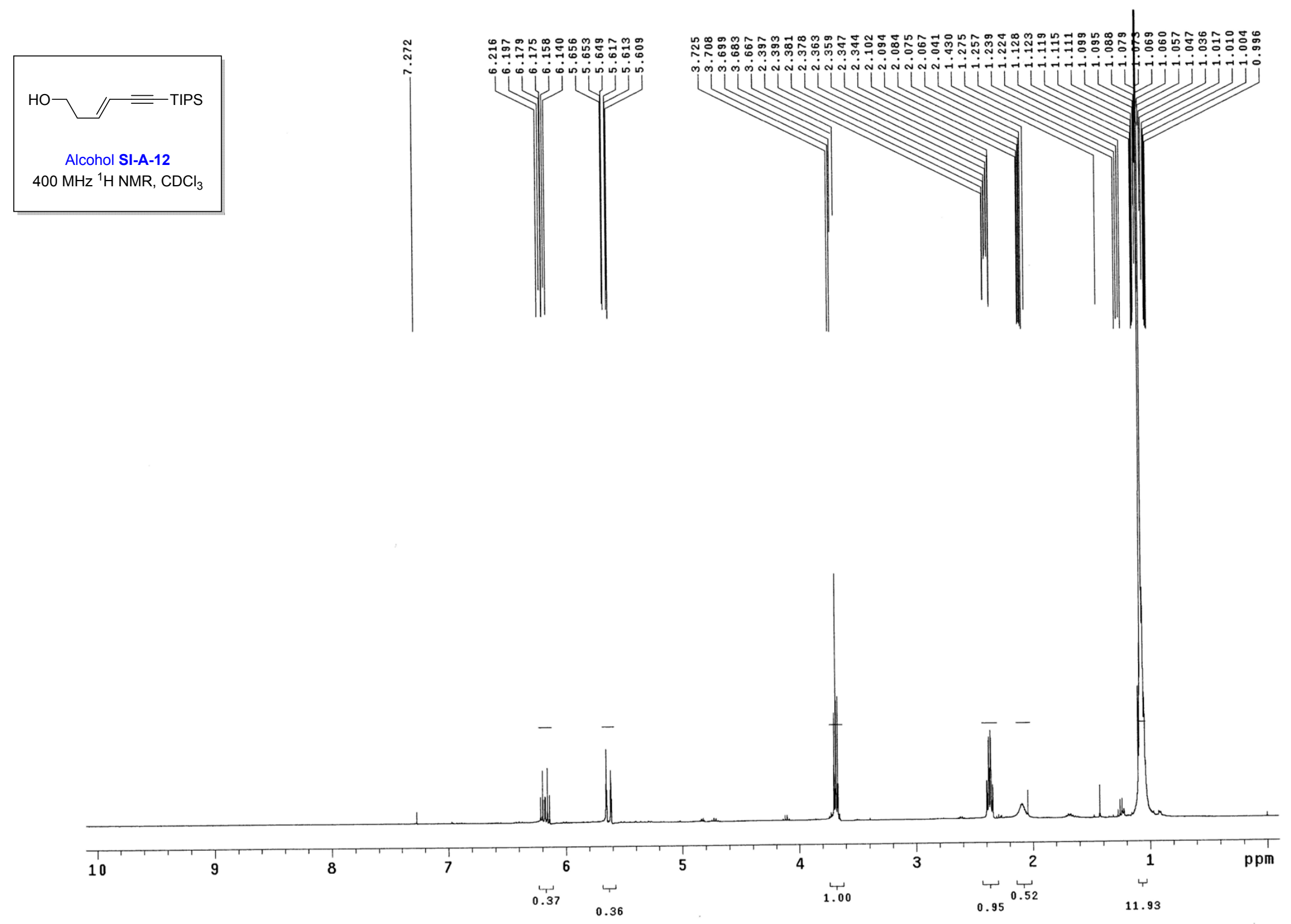

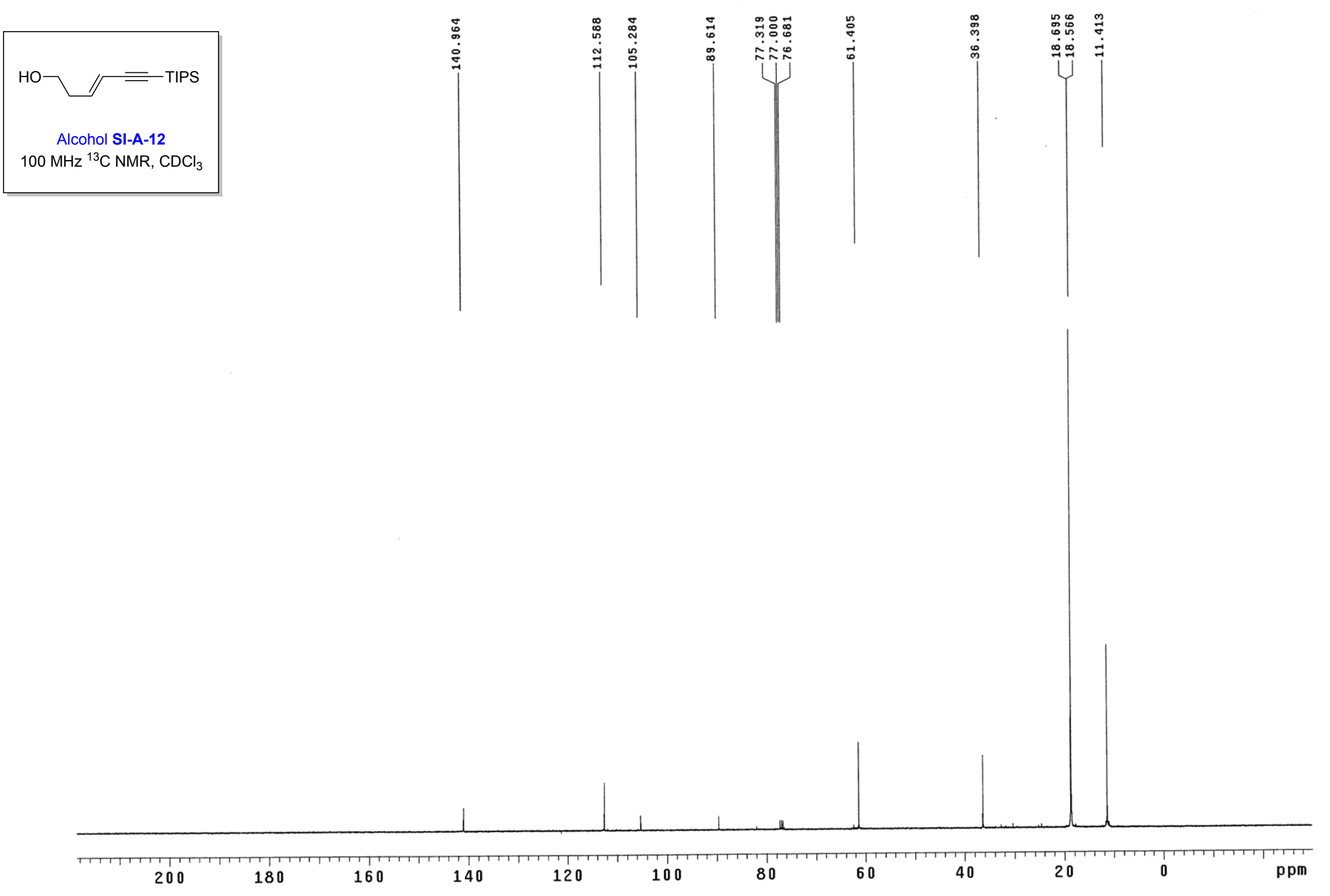

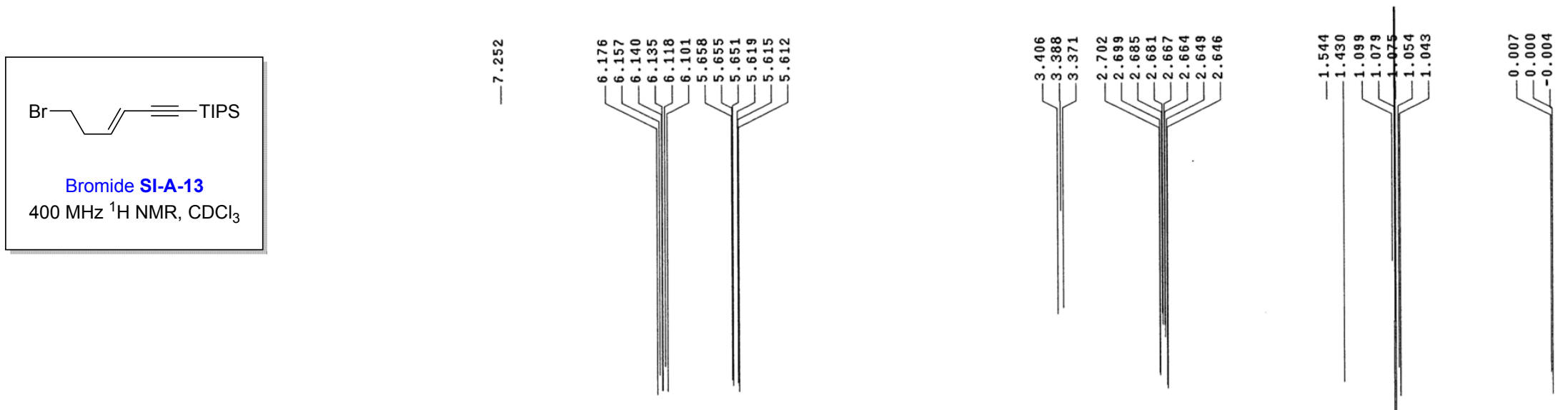


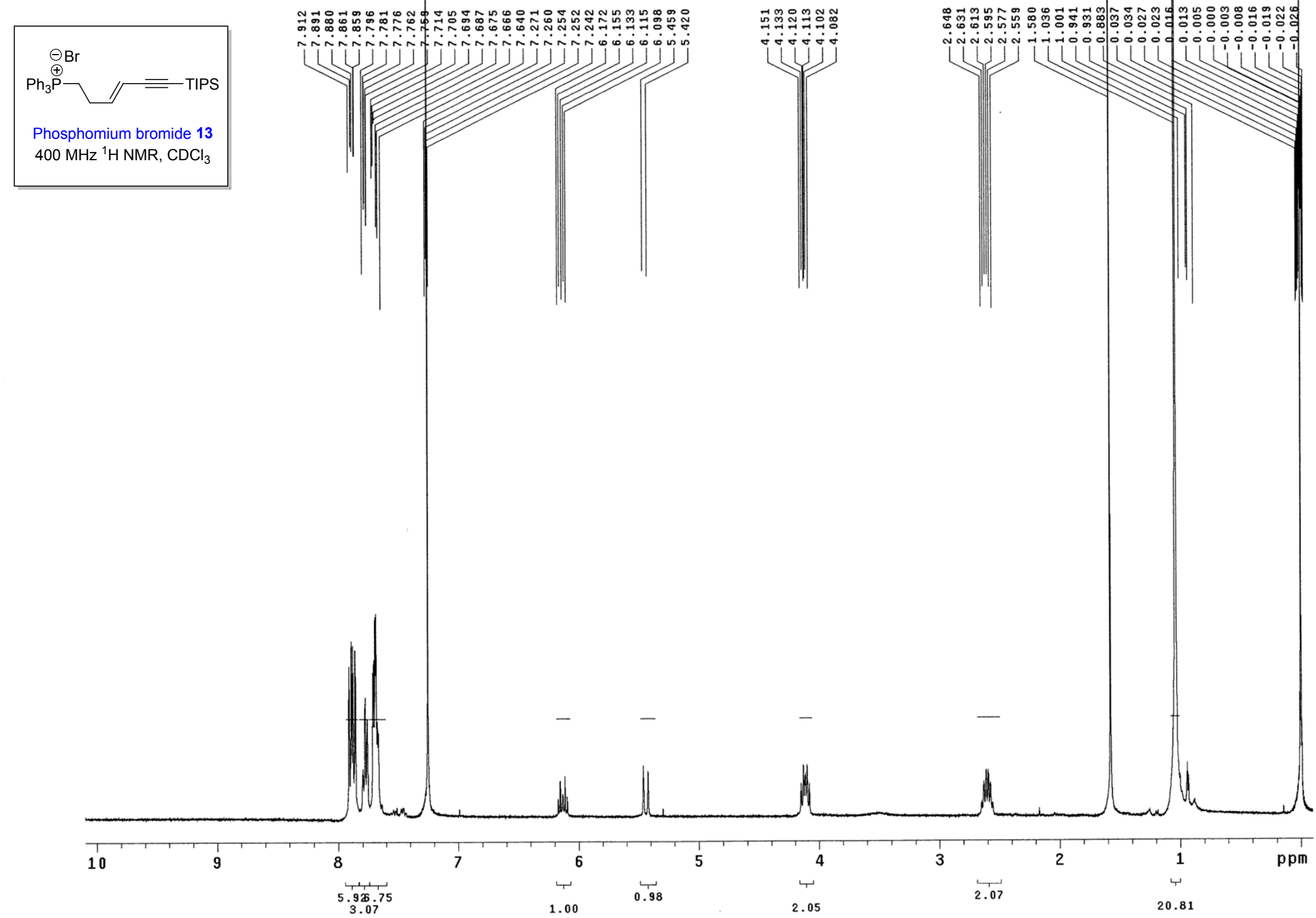




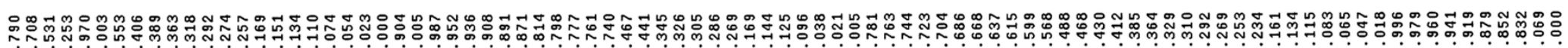

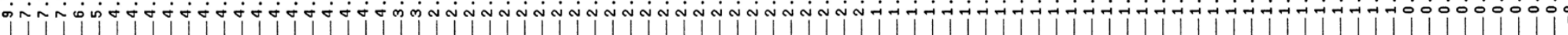

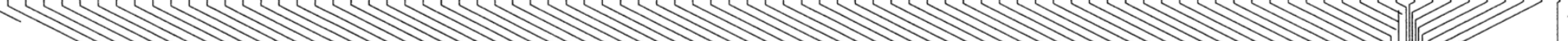
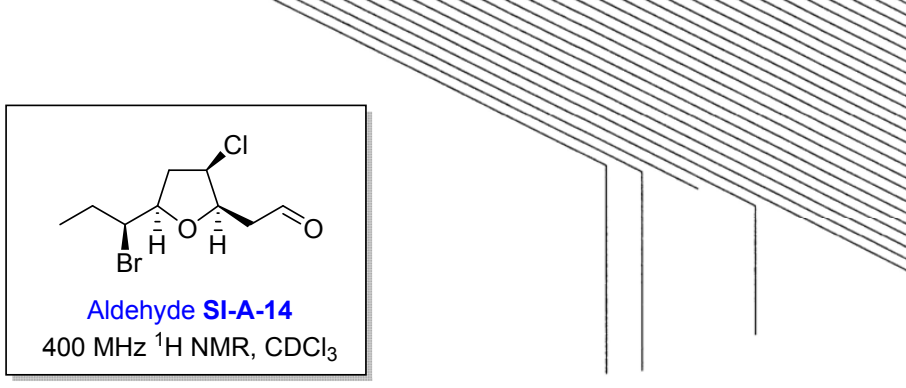

ㄴ.

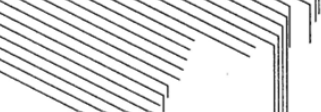
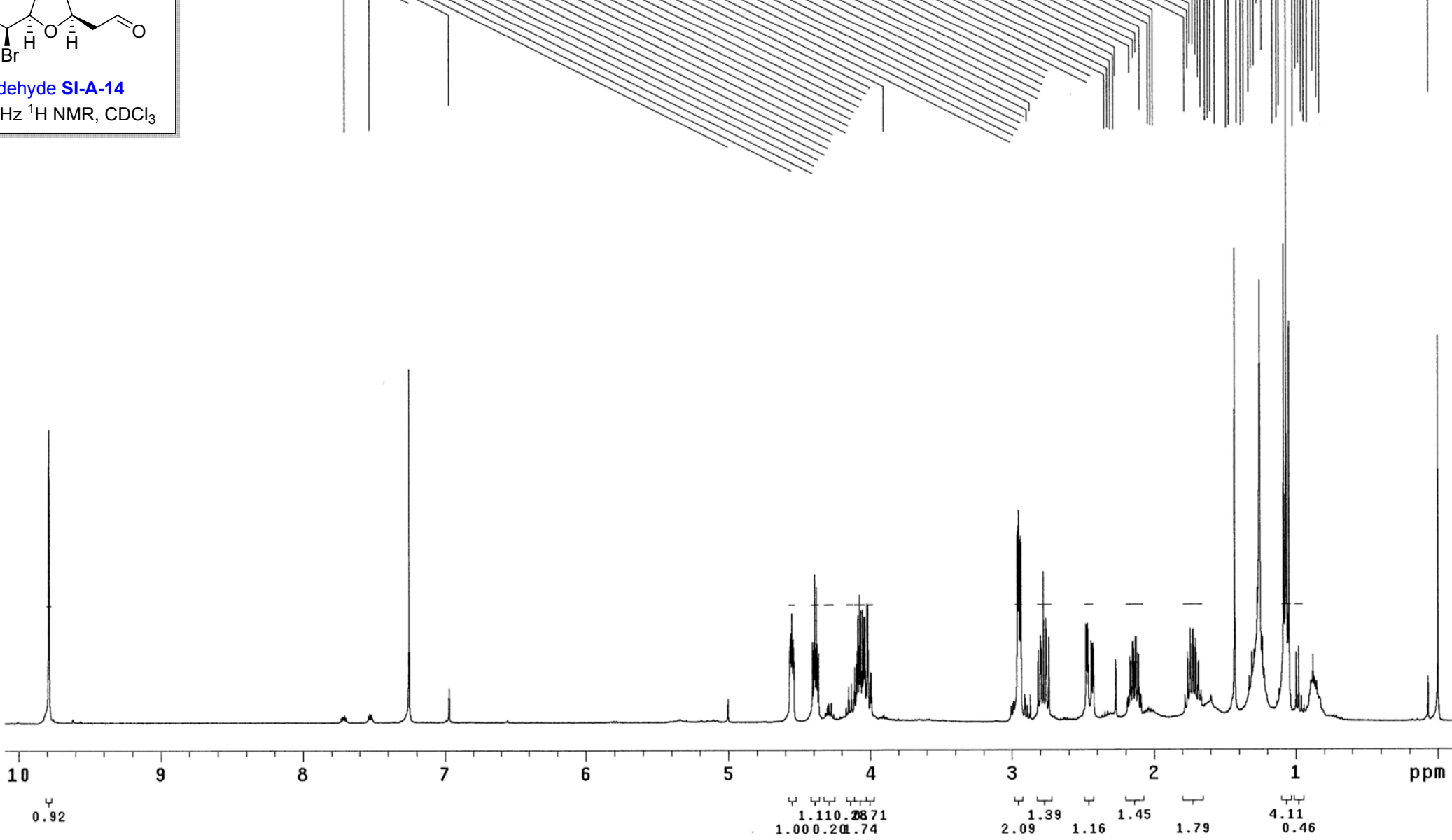


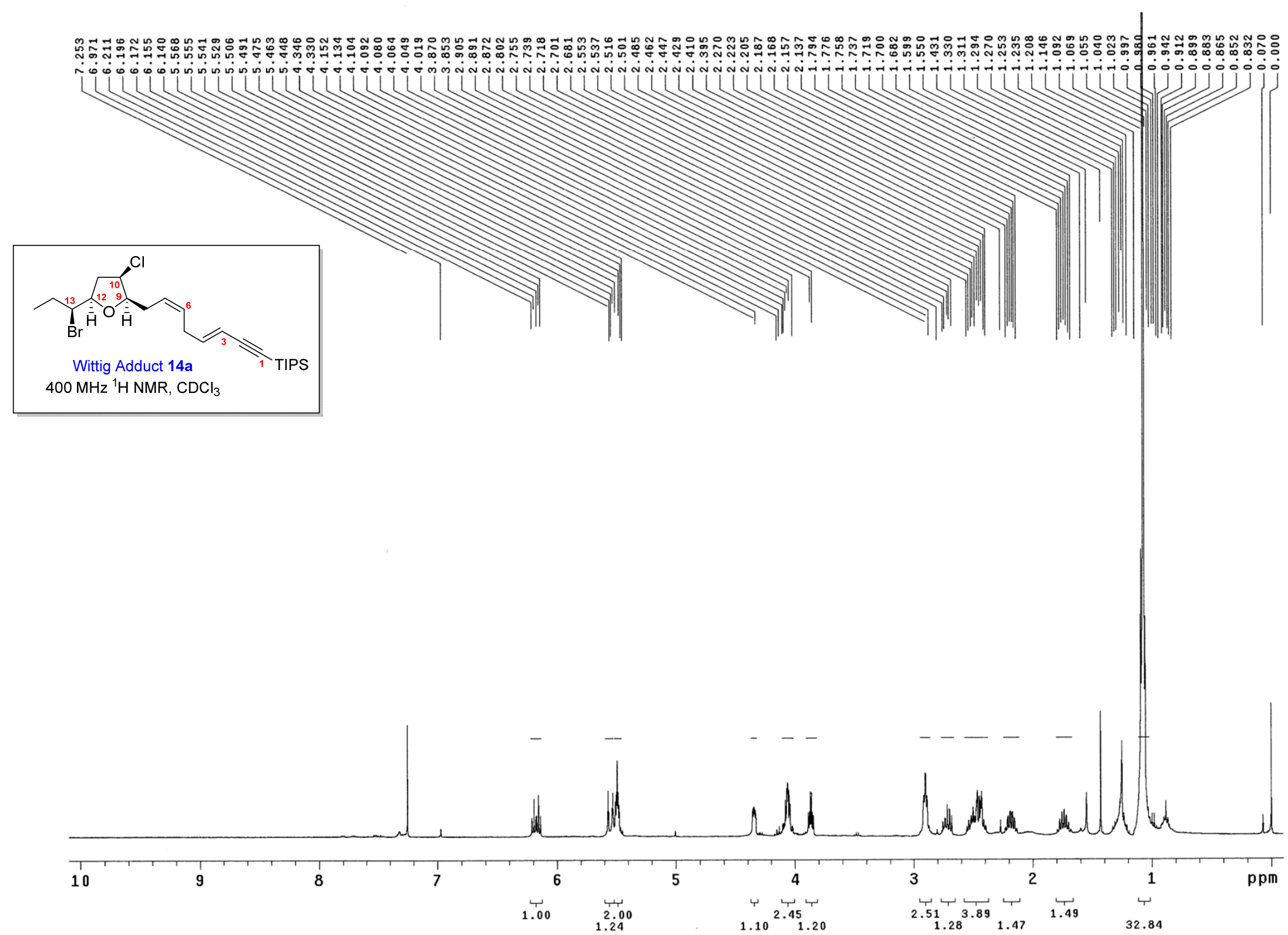




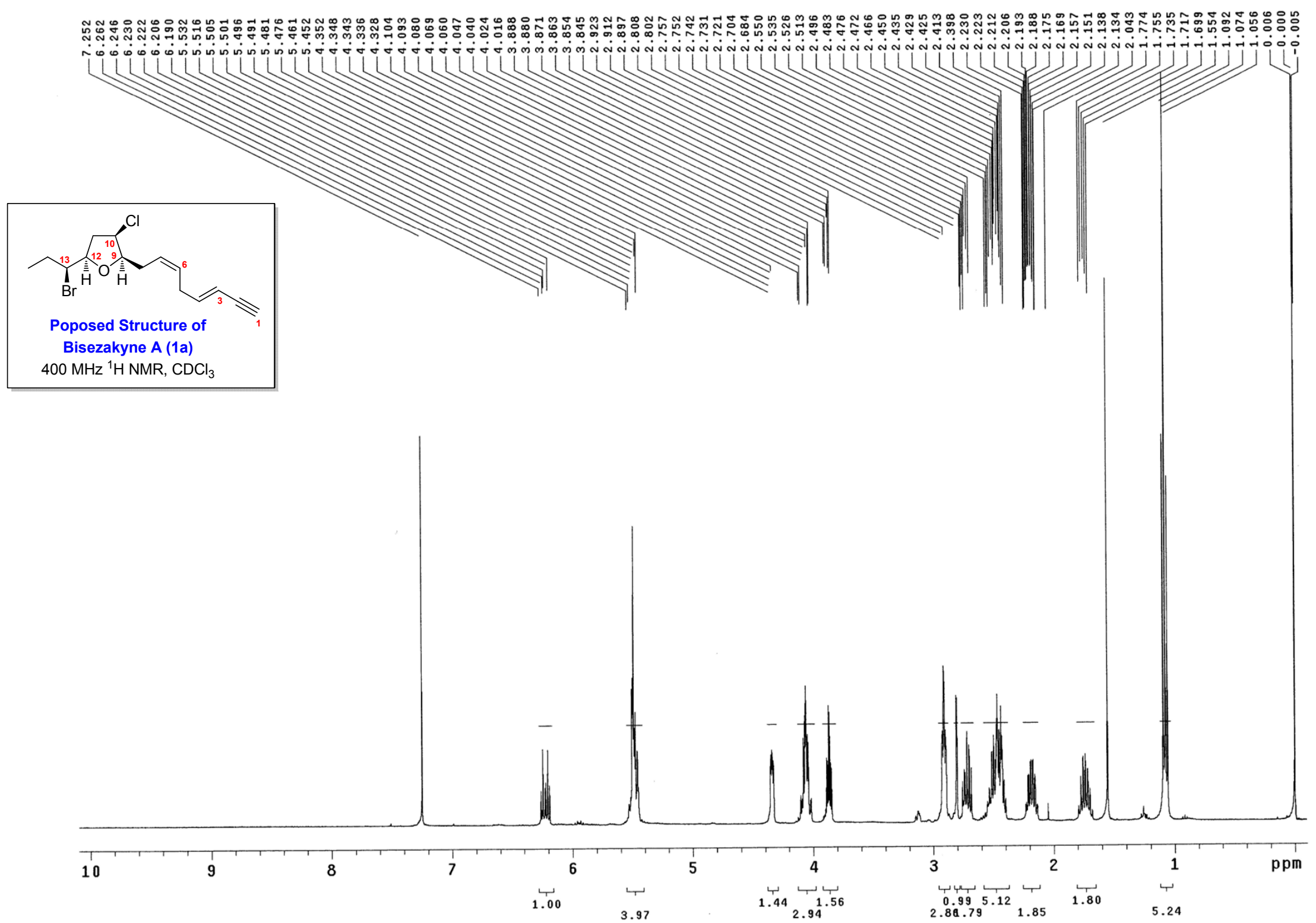



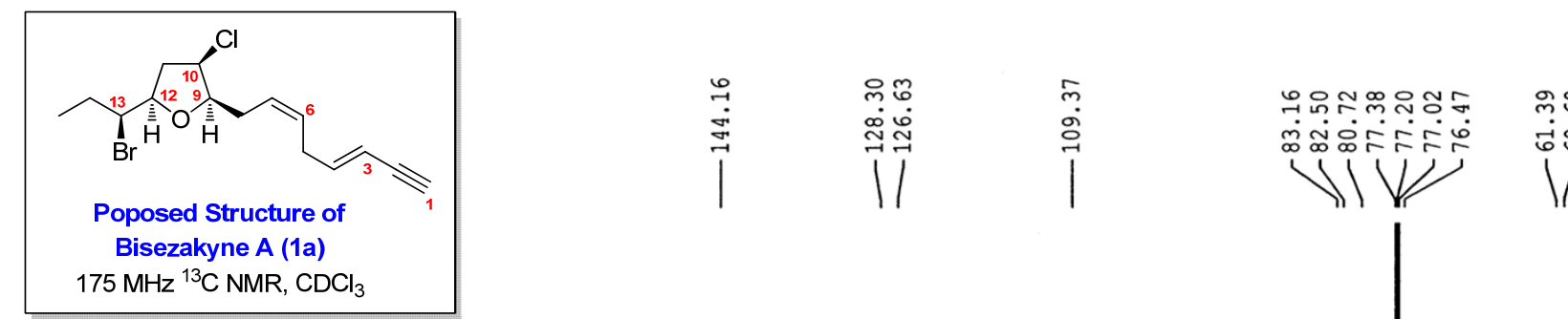

| |

$\mid$
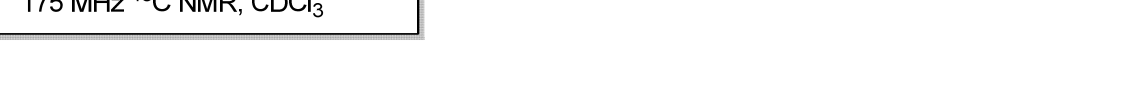


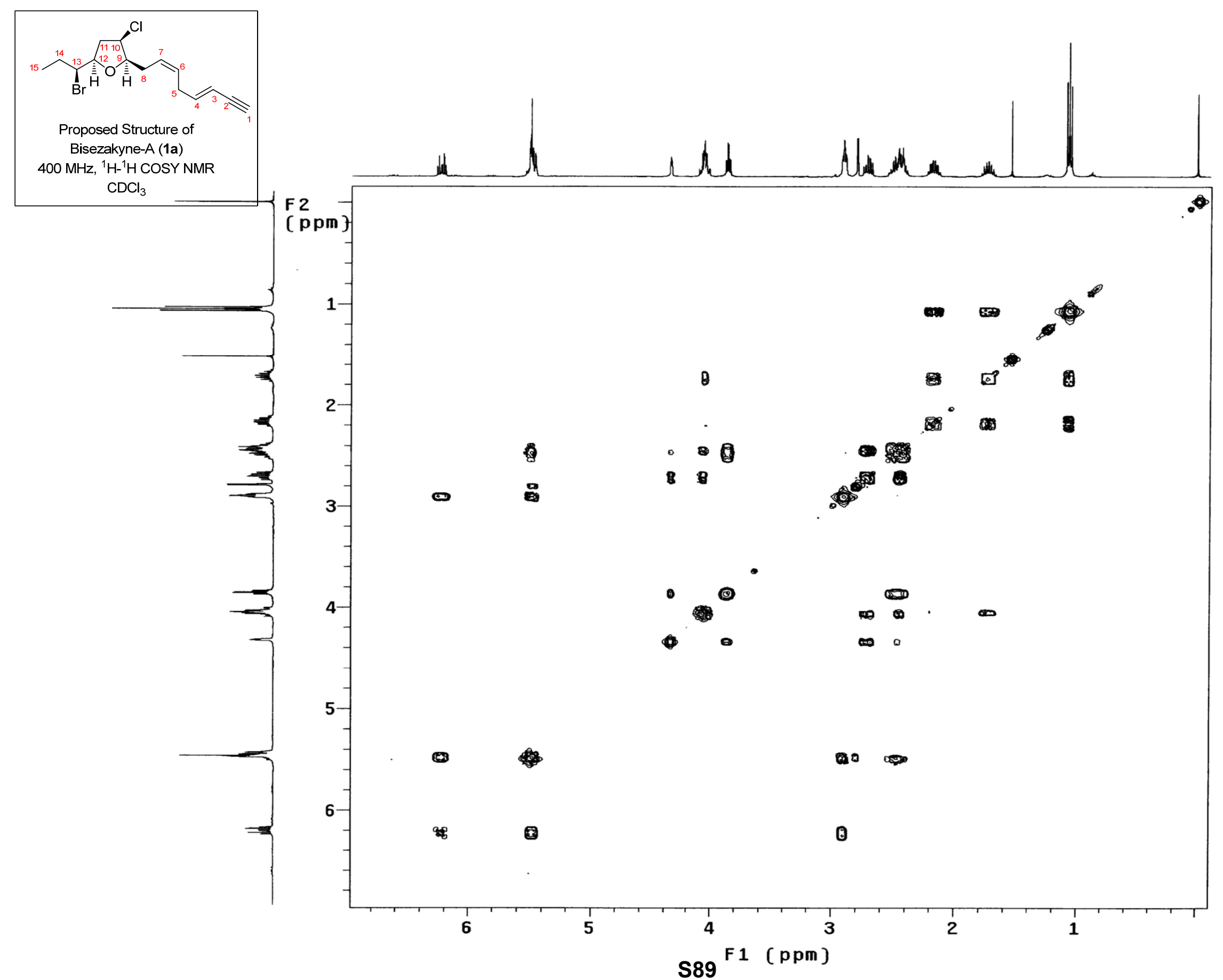




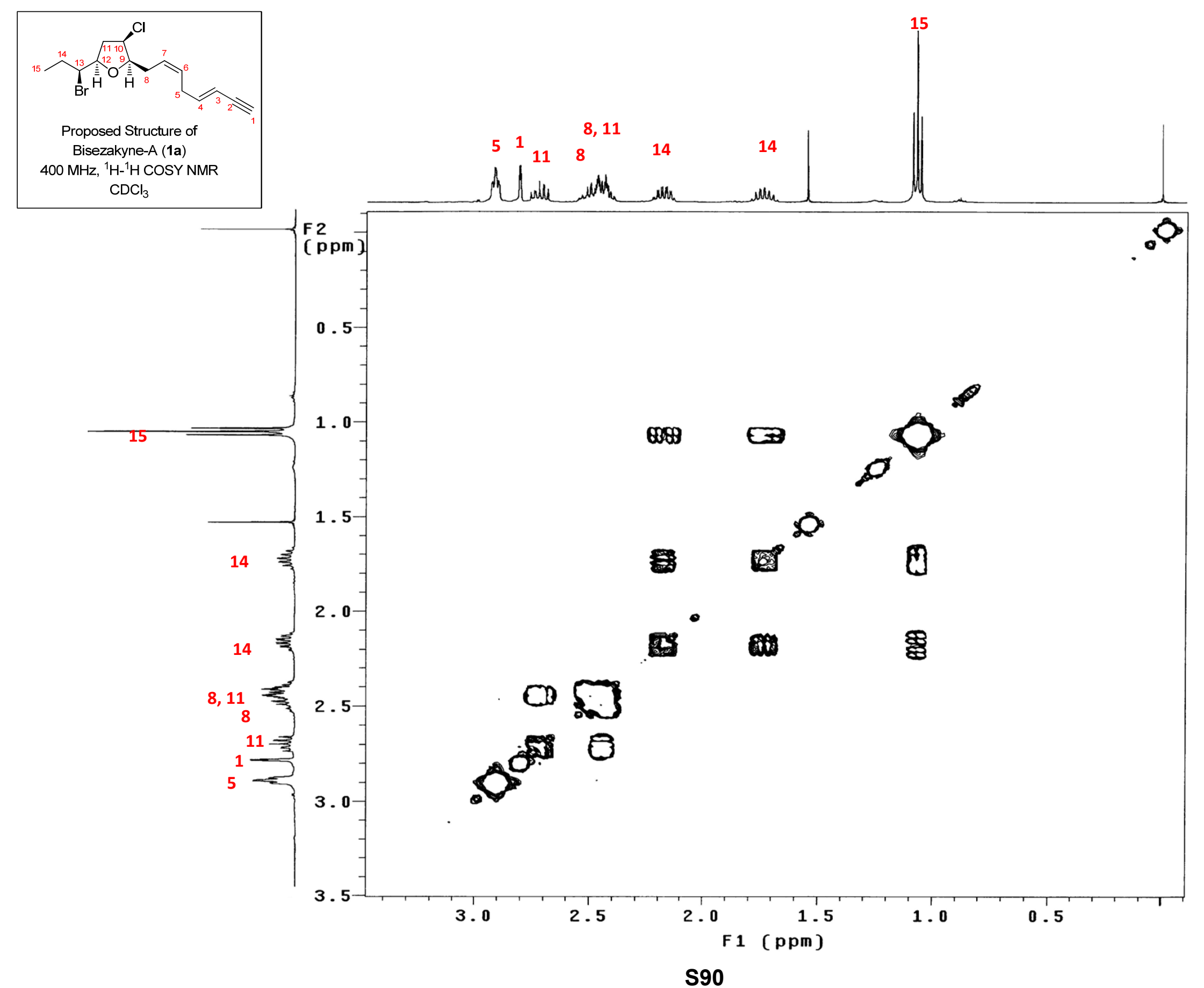




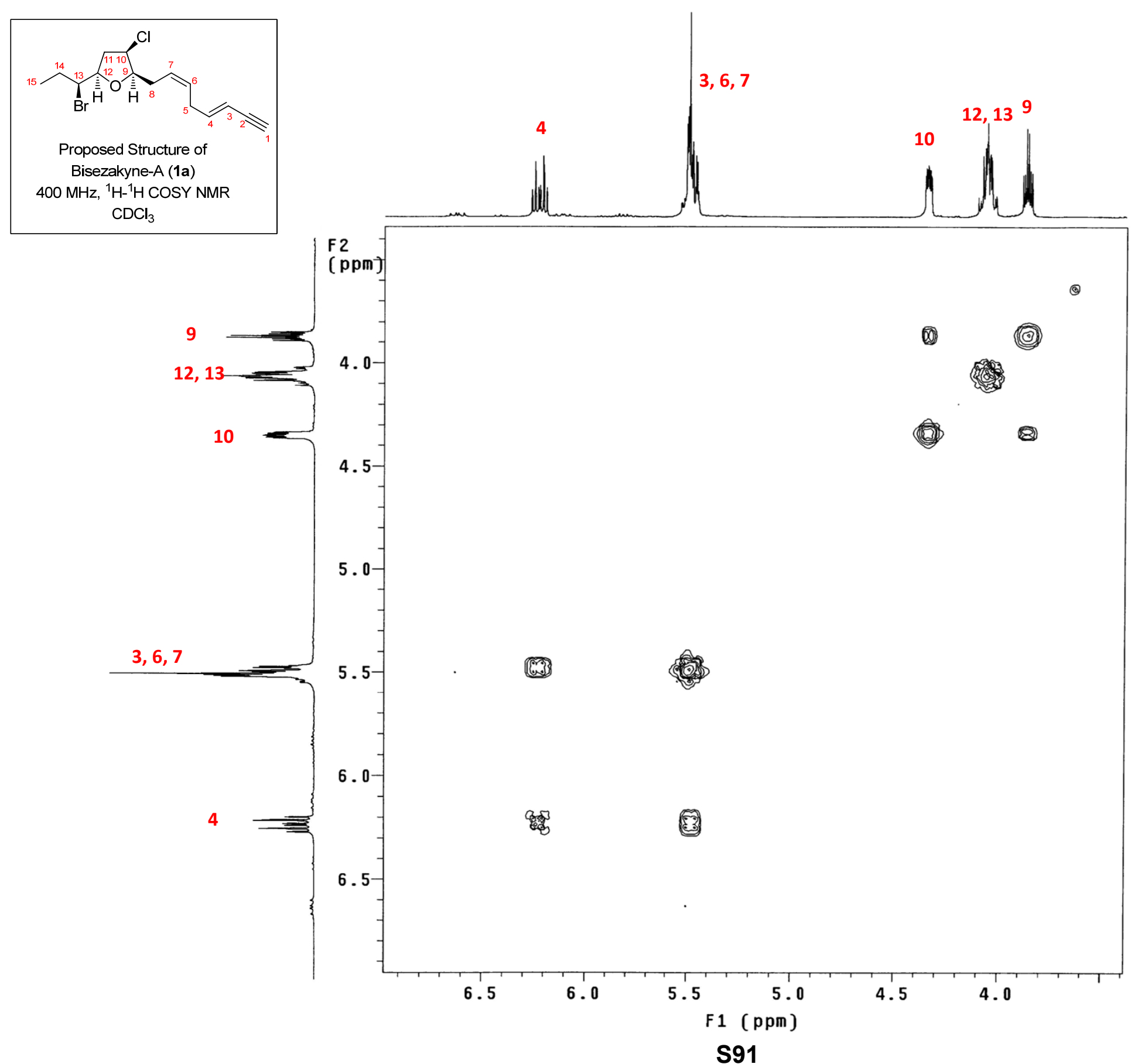




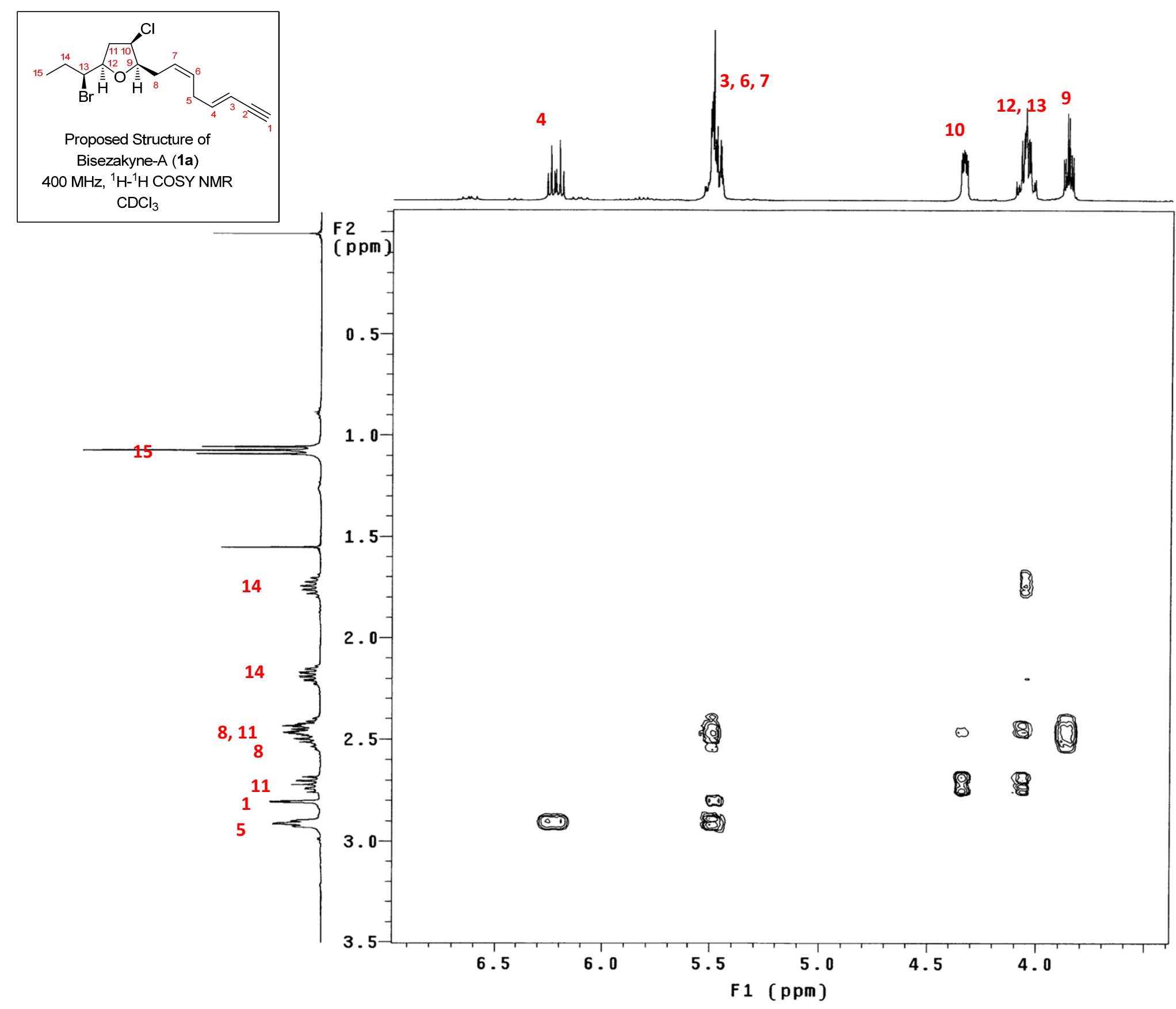

S92 


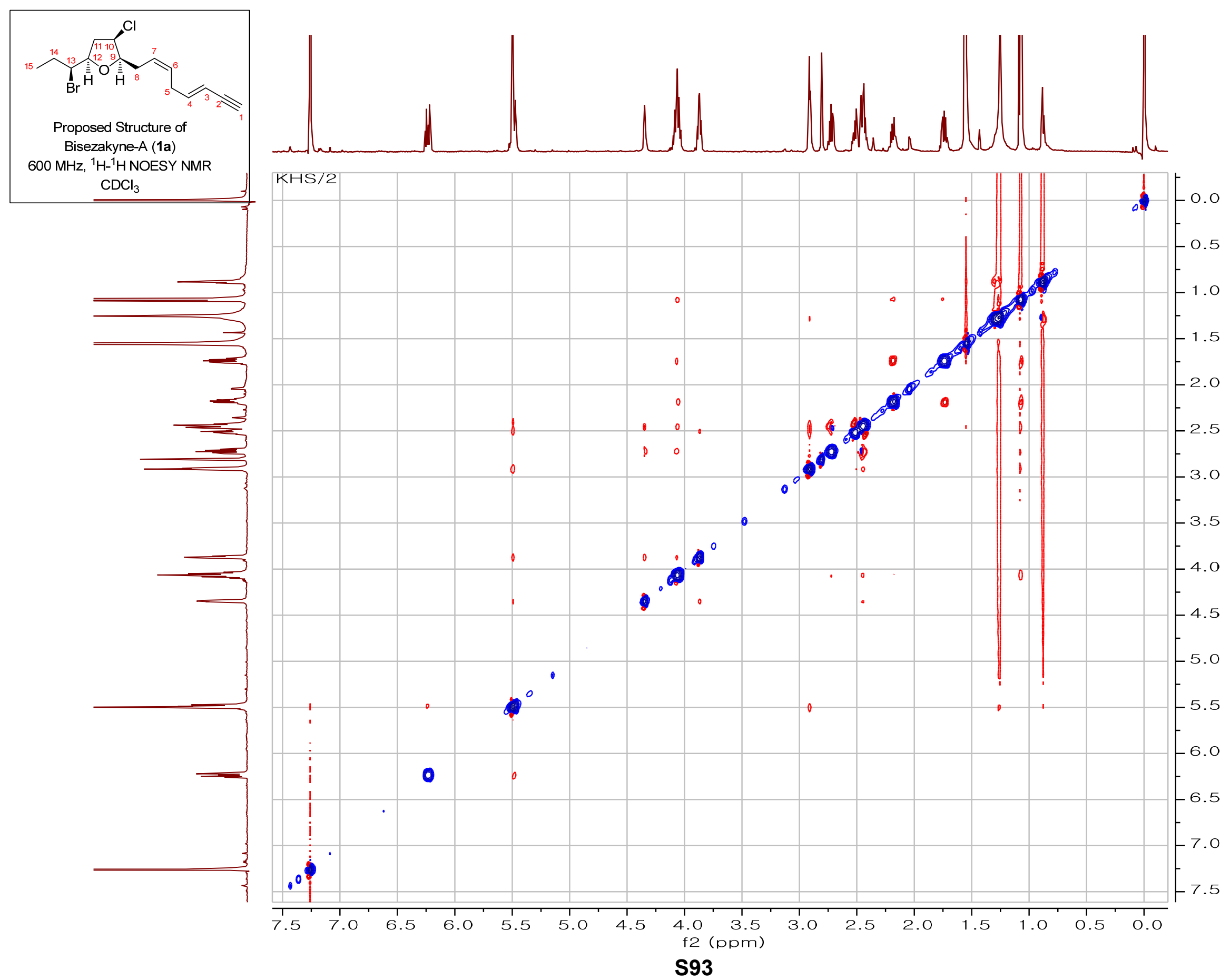




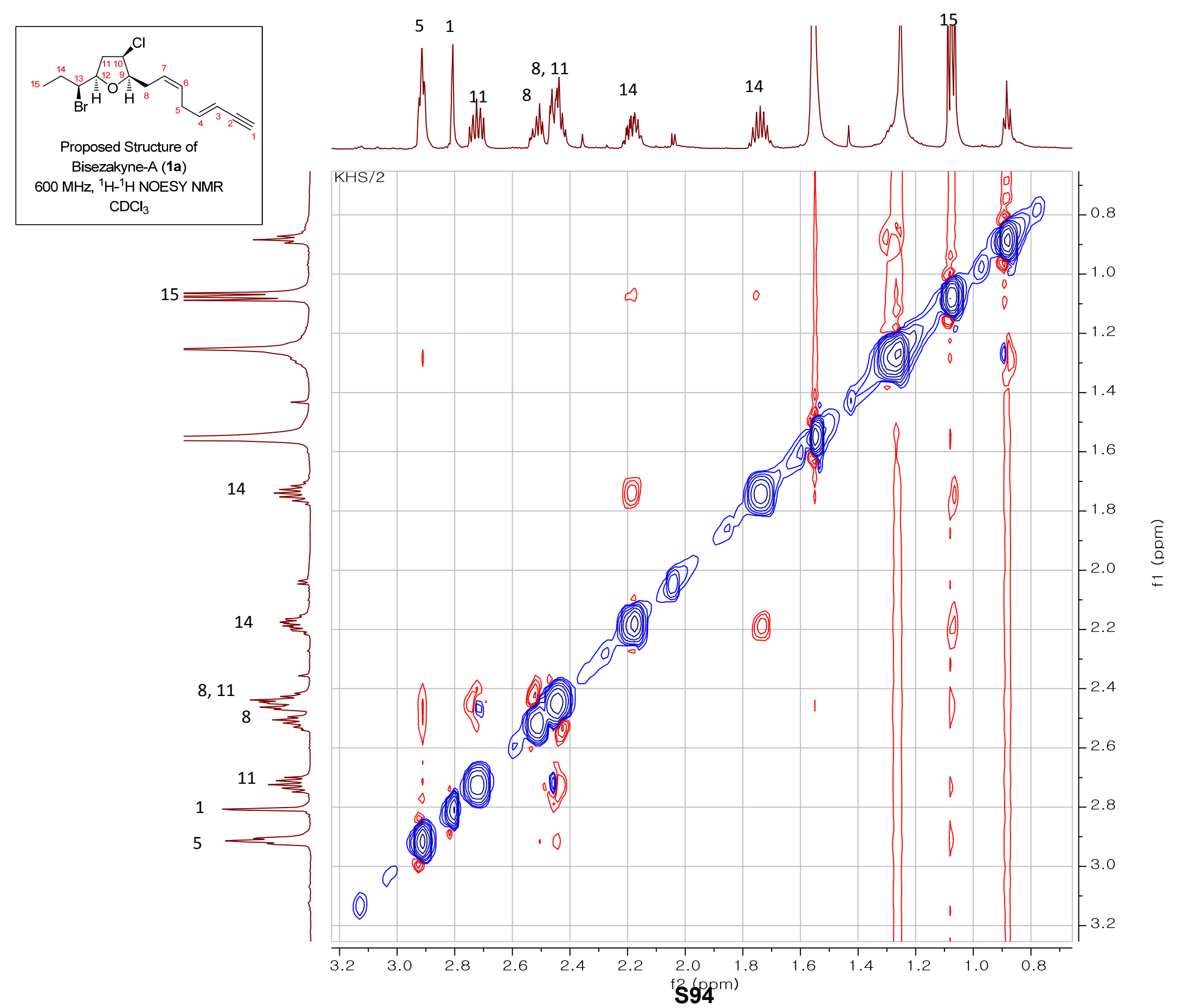




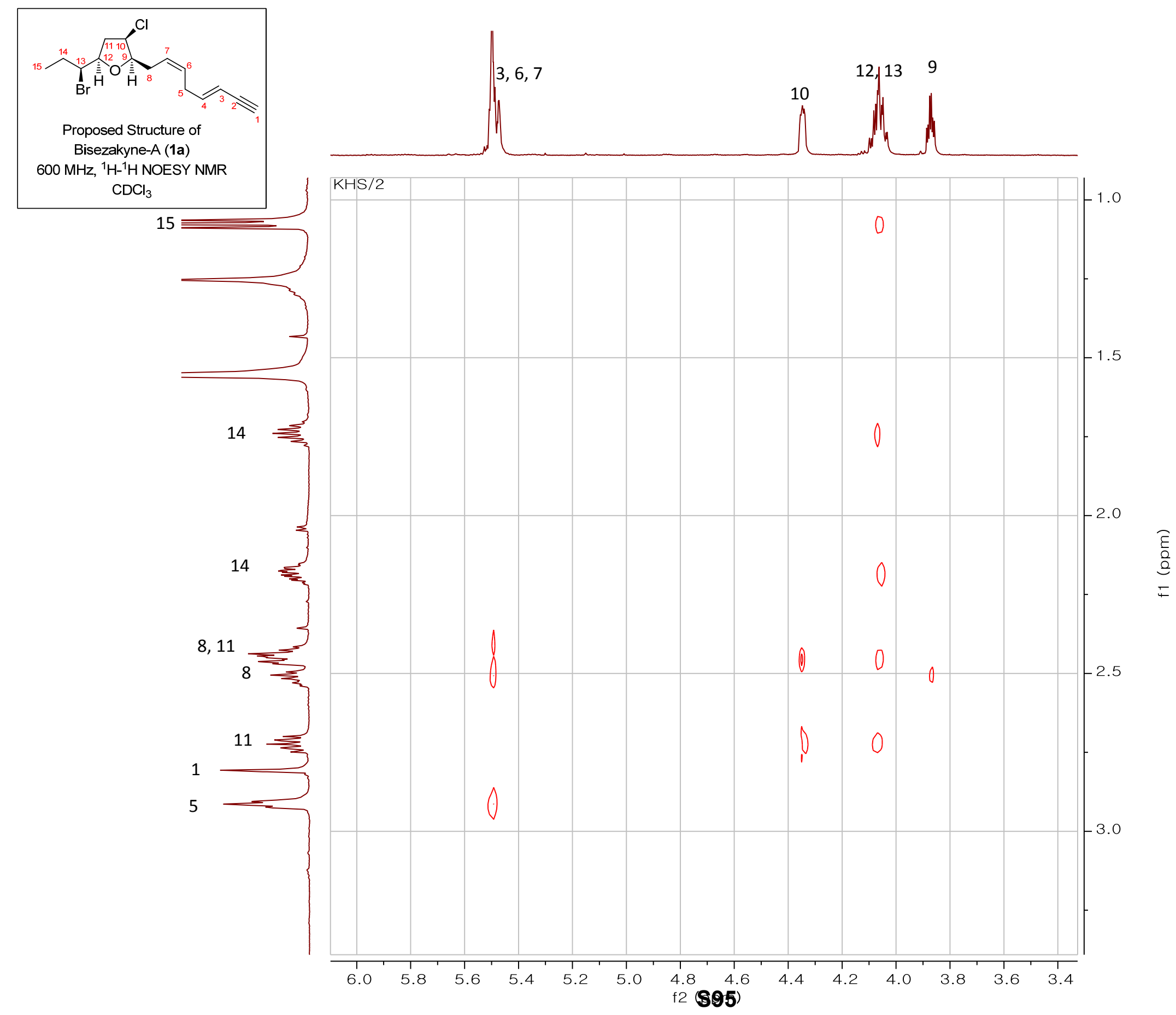




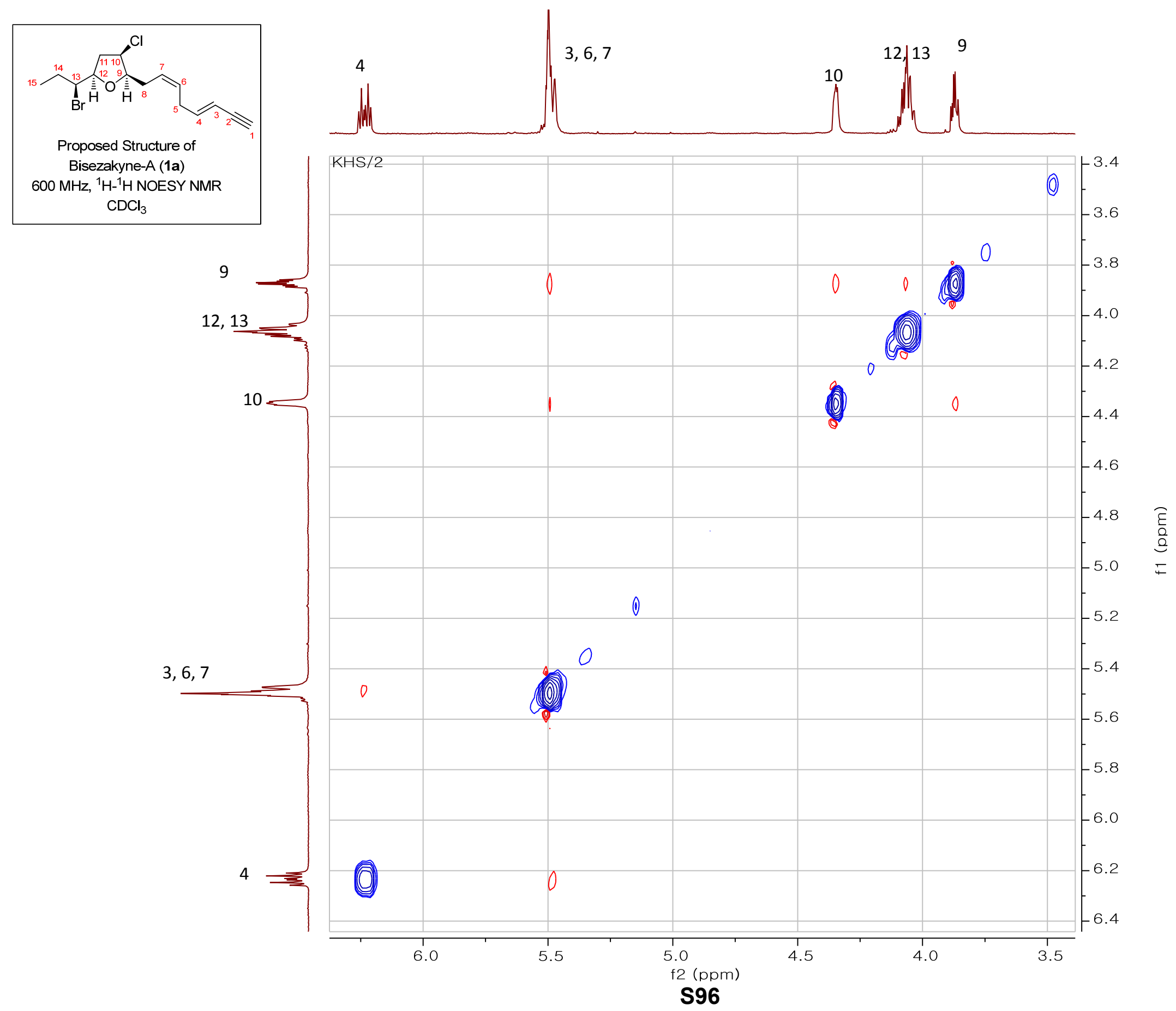




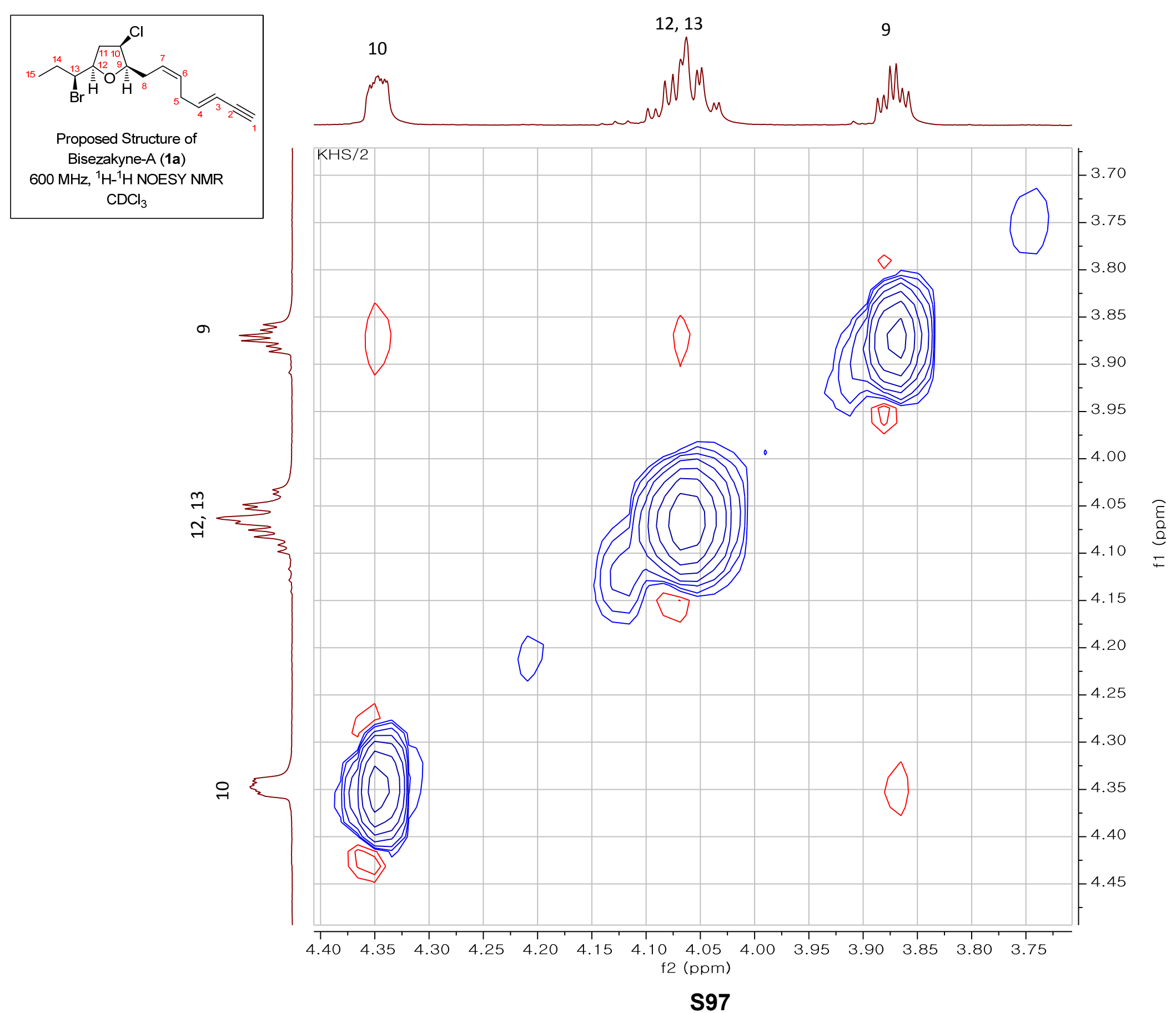




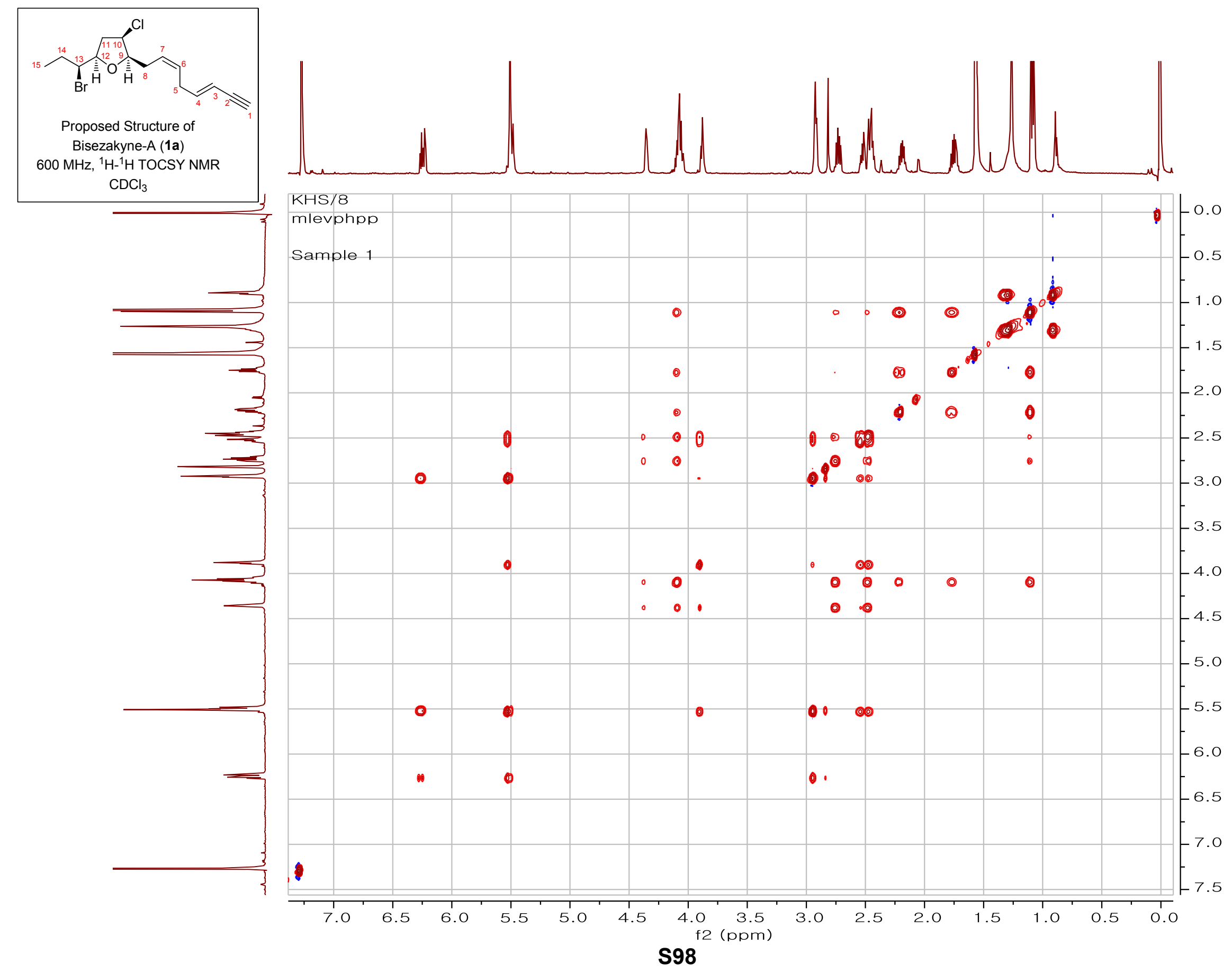




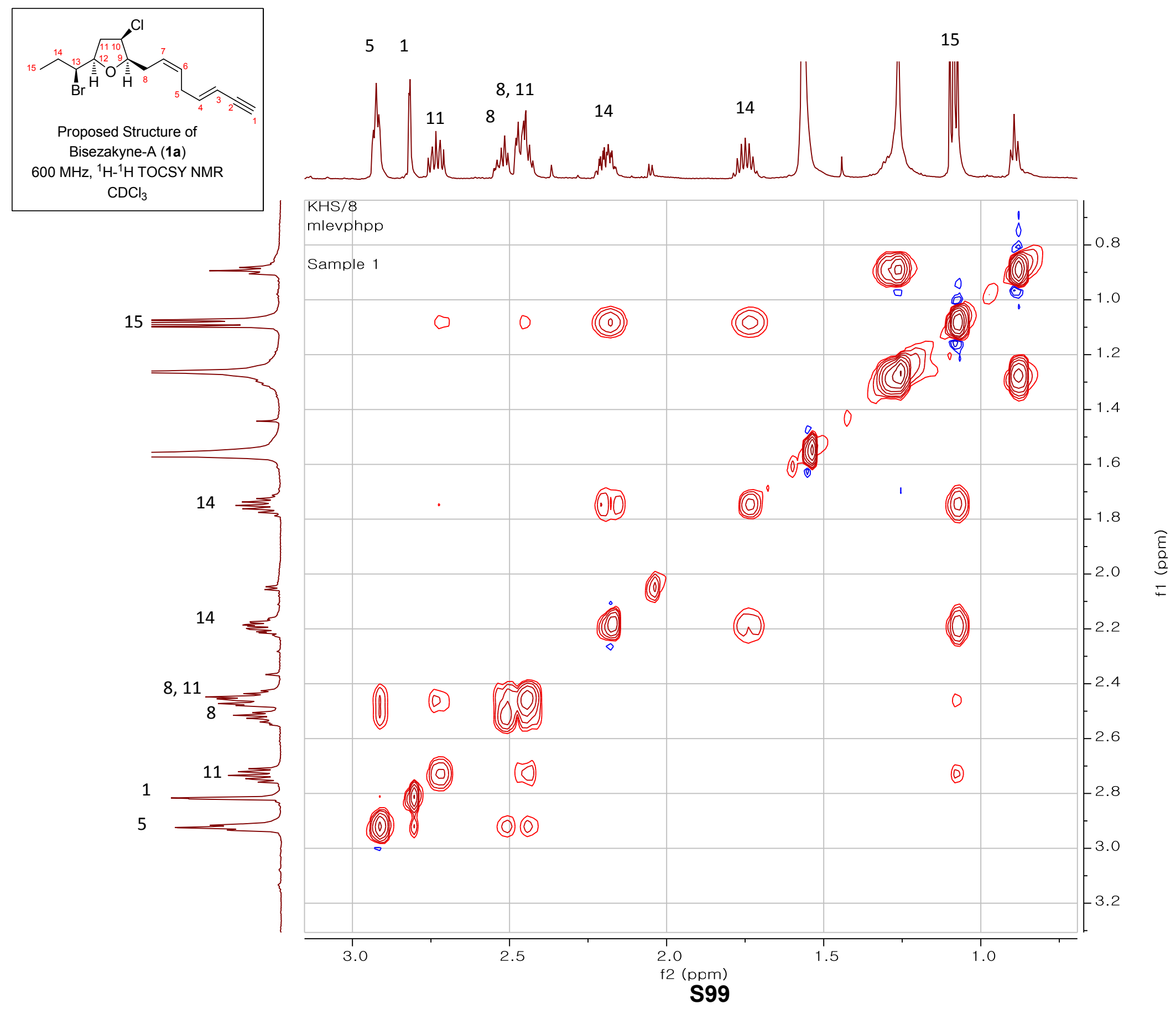




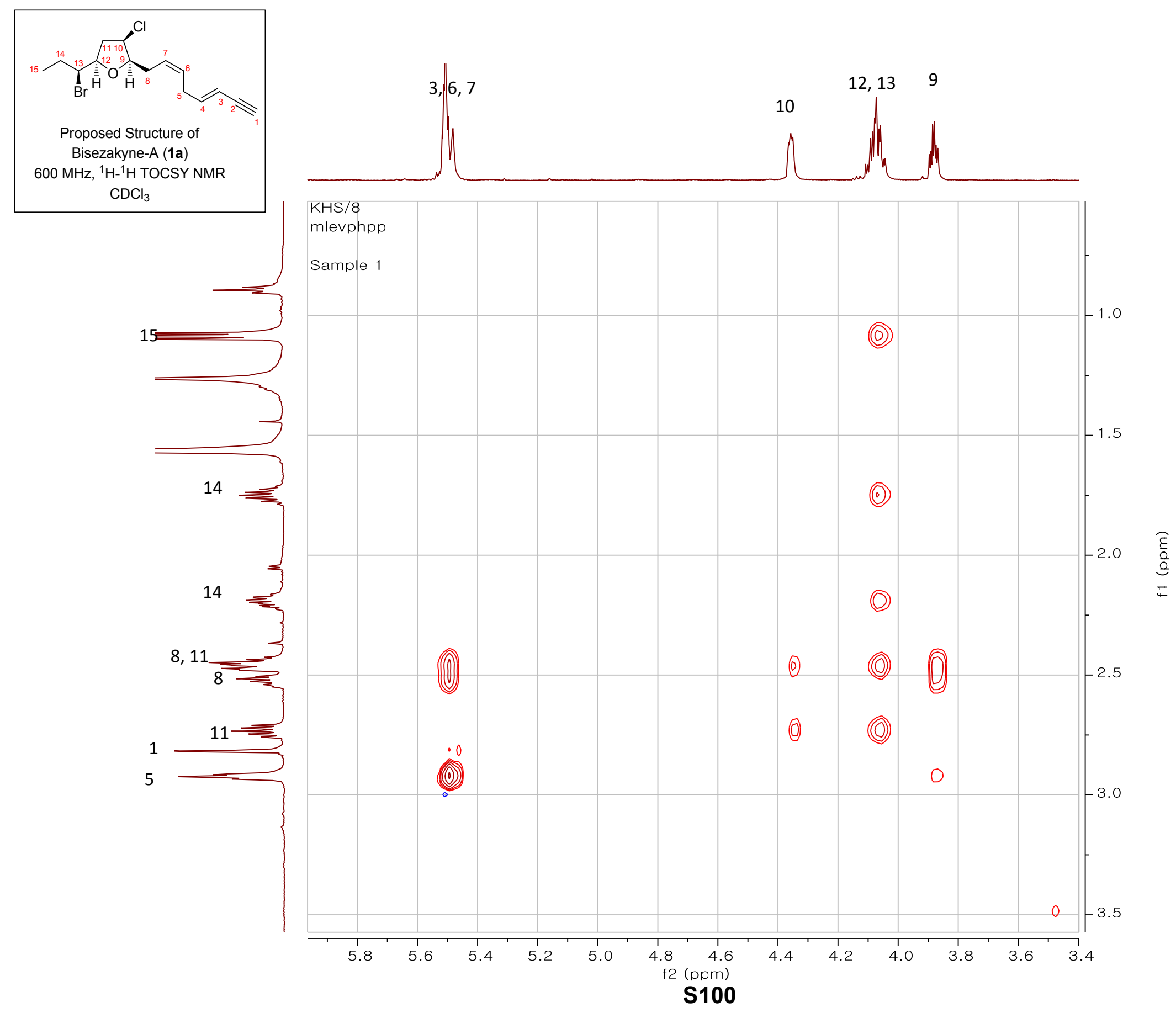




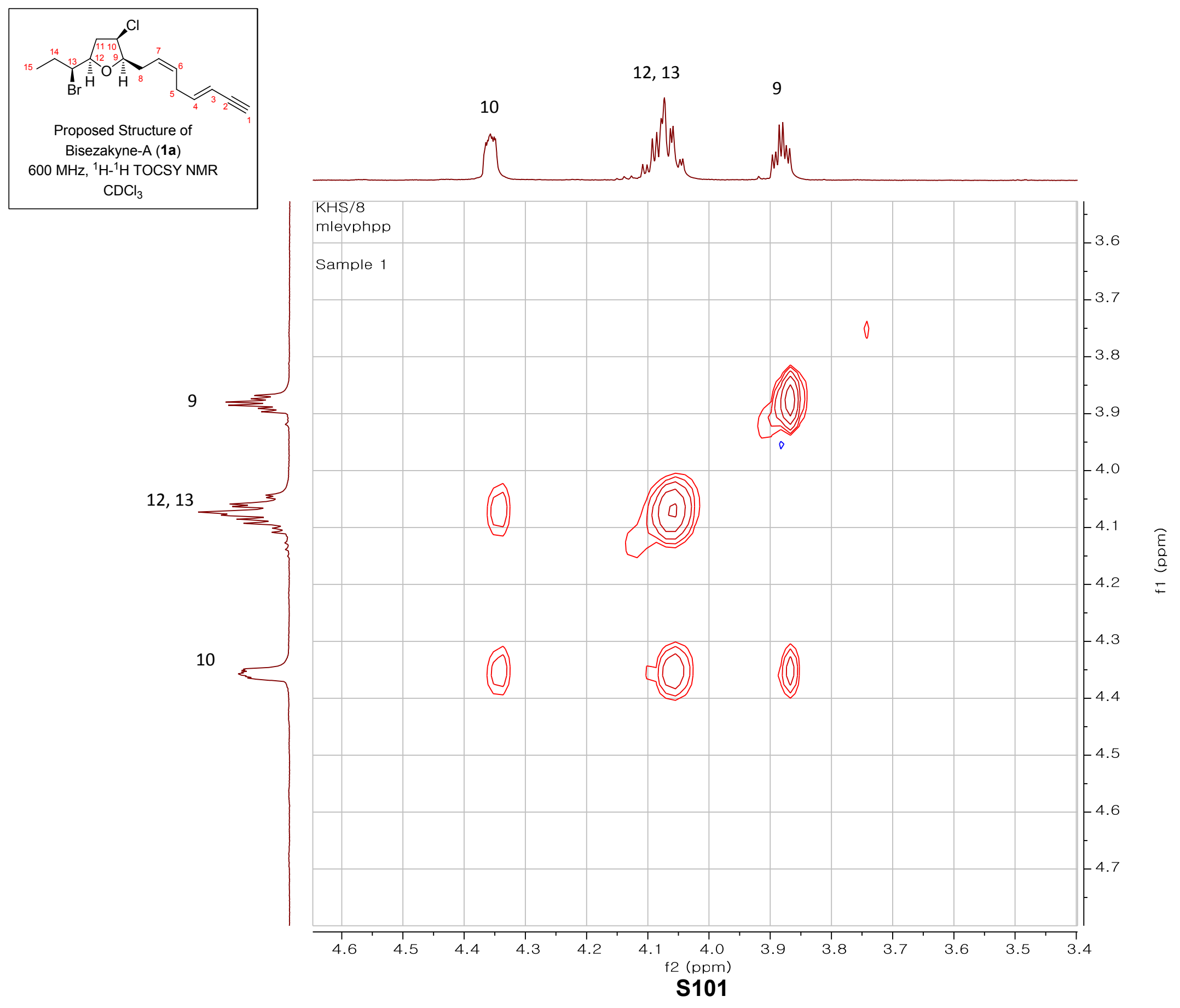




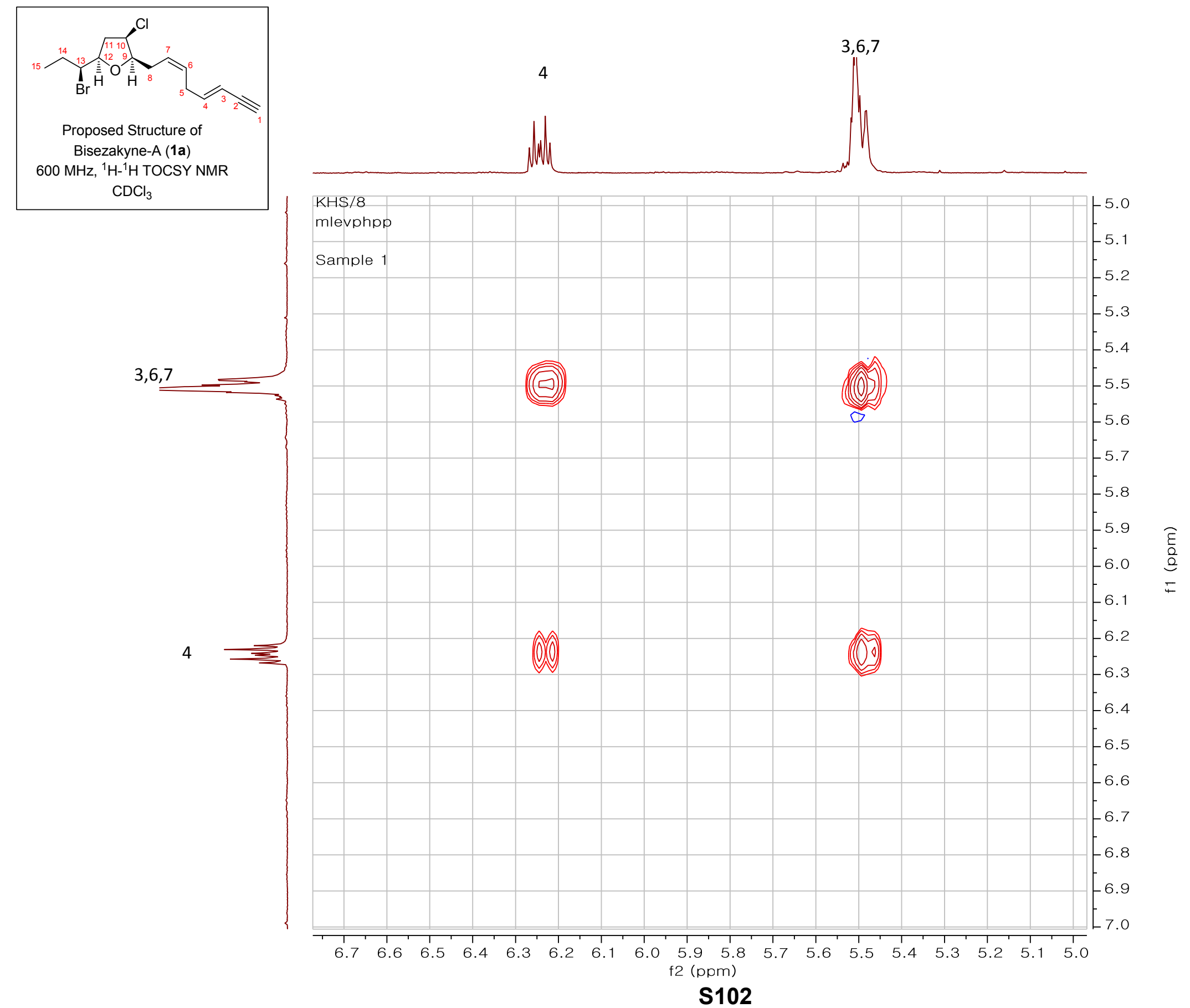




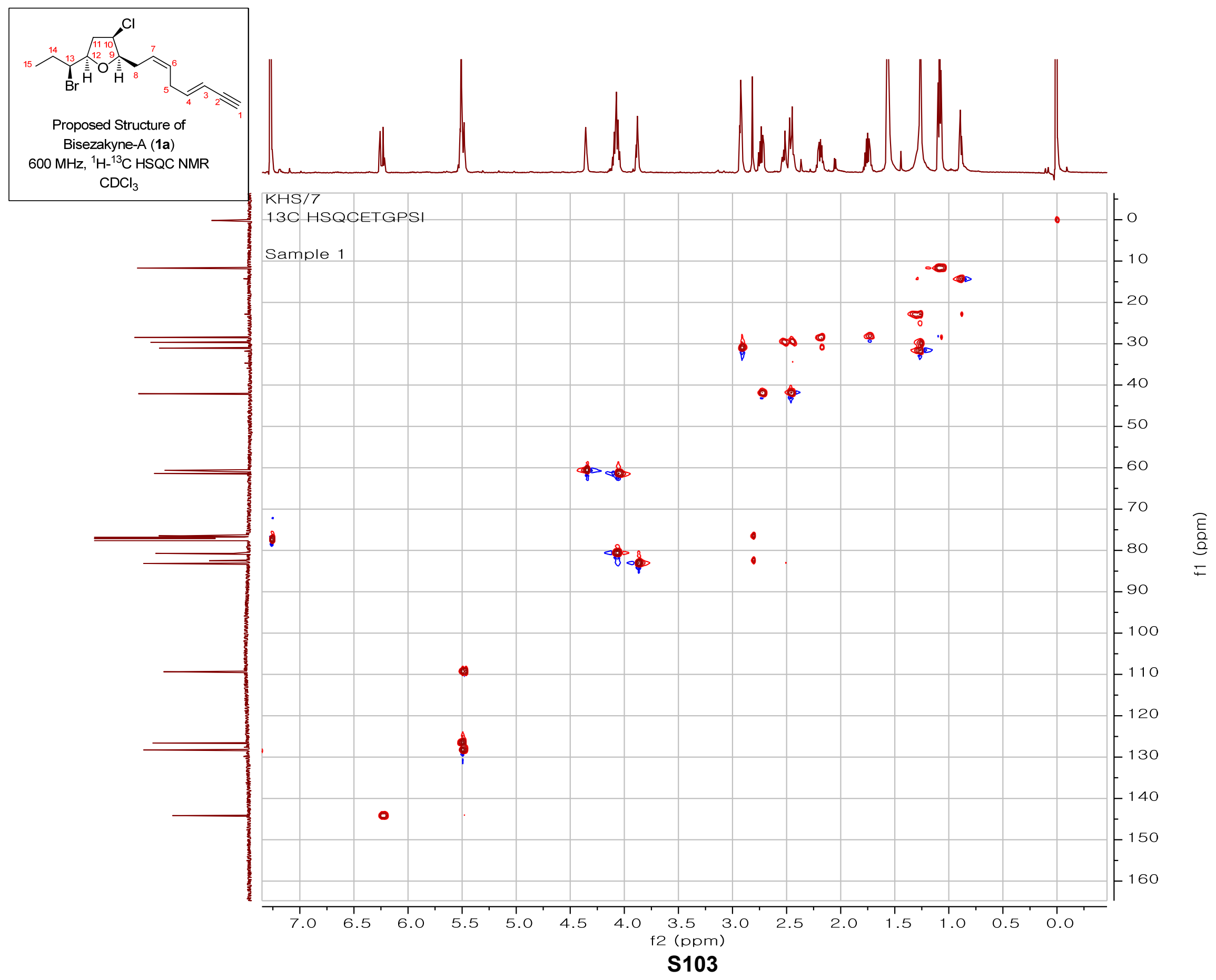




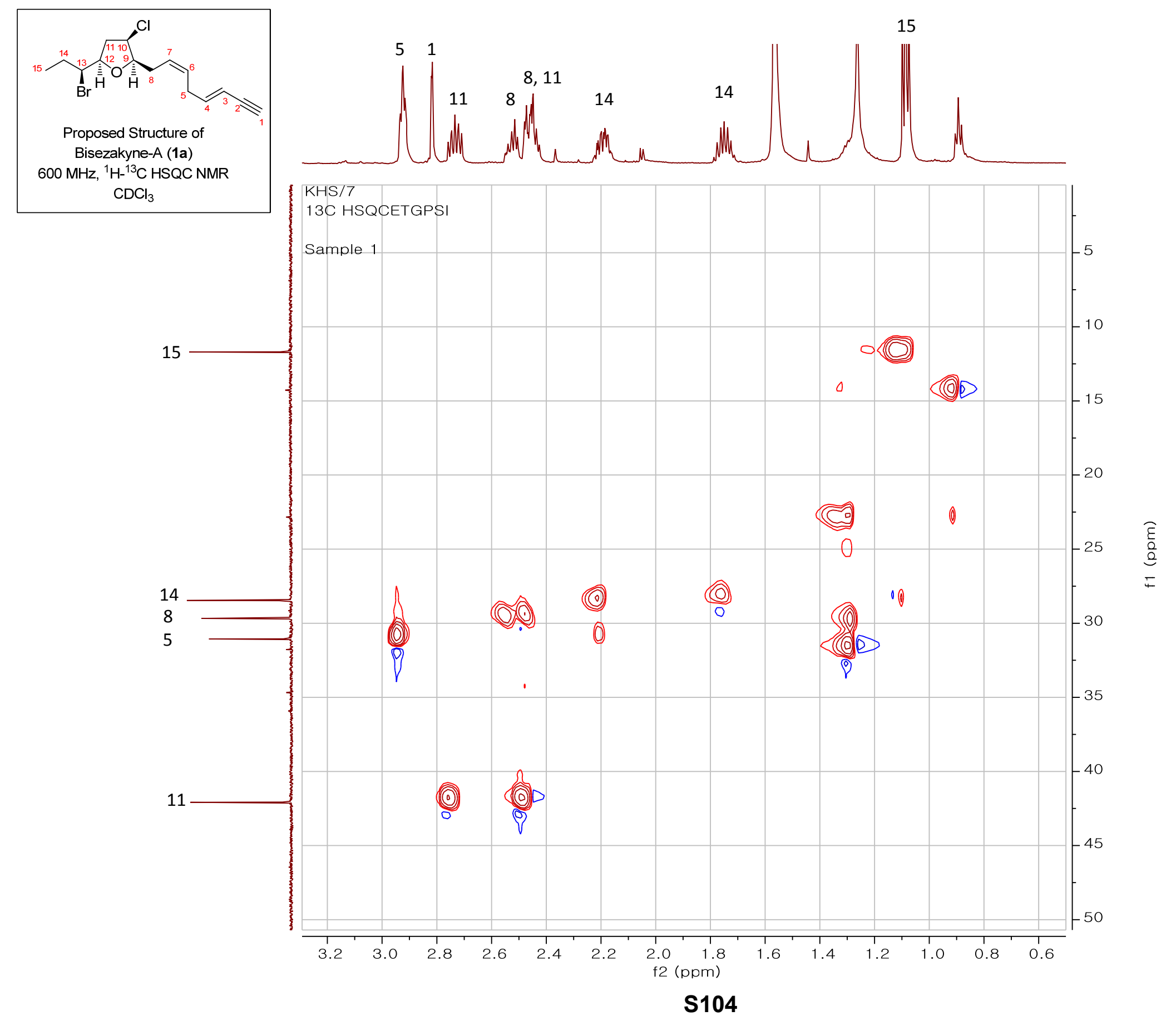




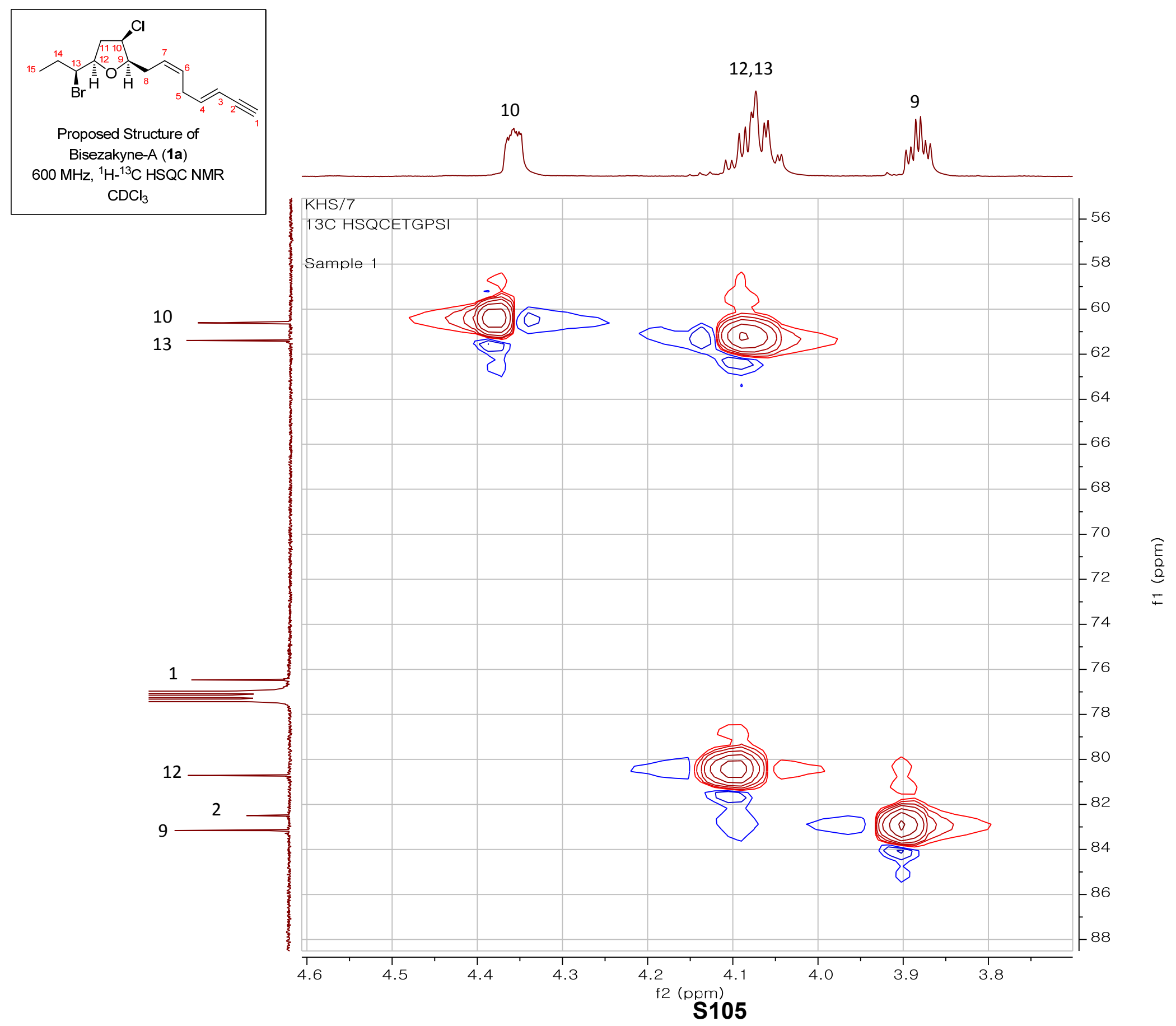




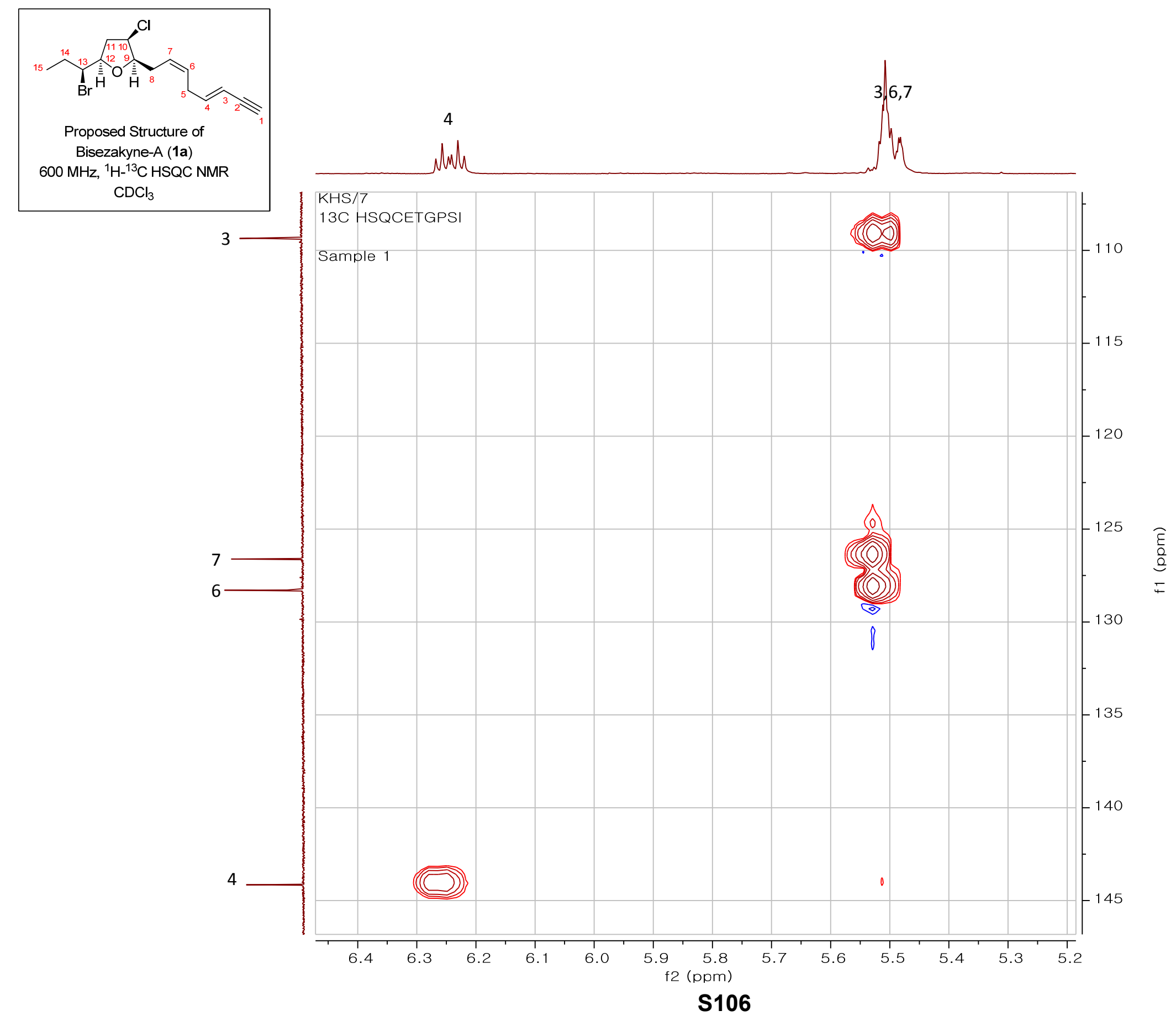




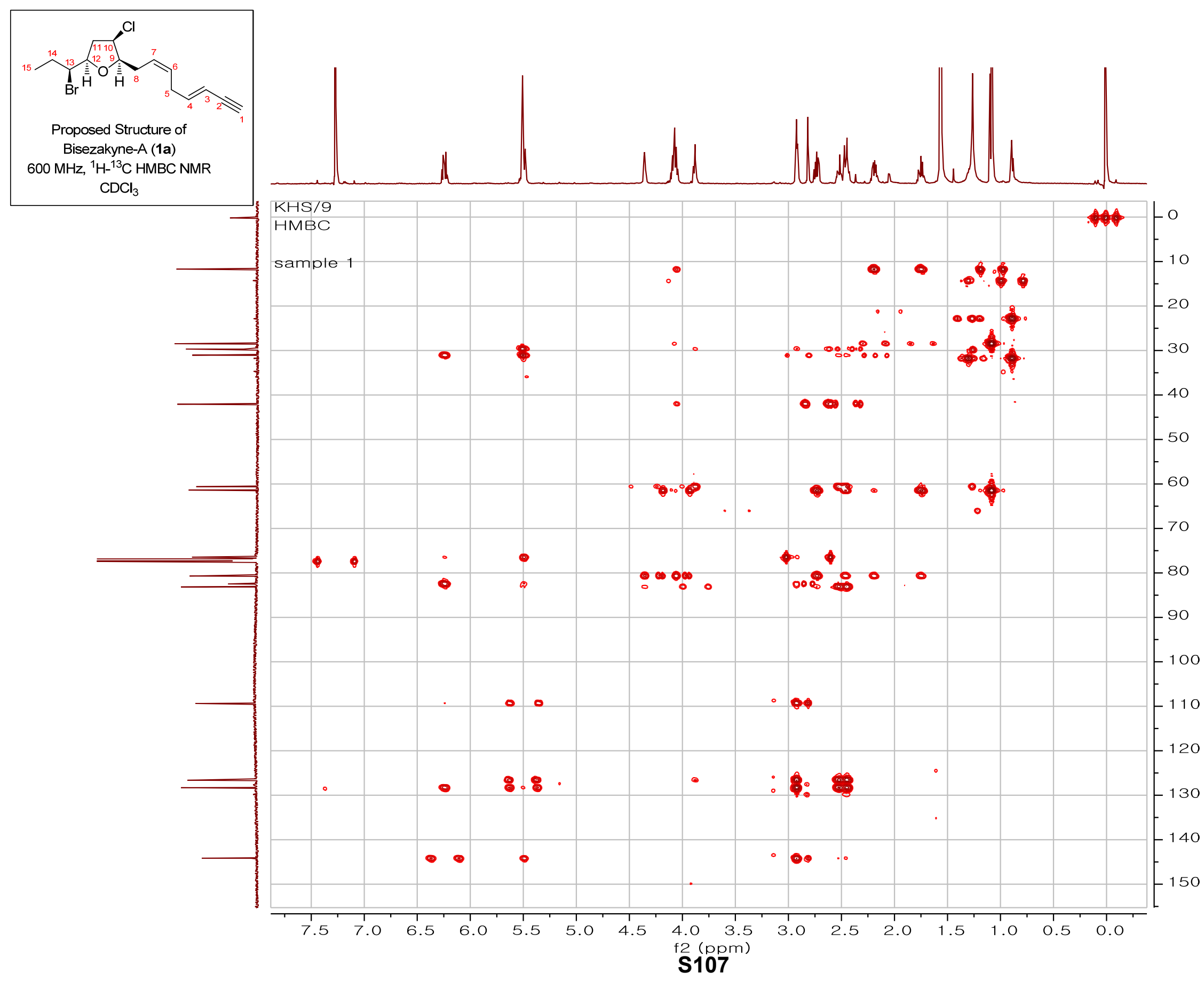

$\bar{\varepsilon}$
a
$\overline{4}$ 


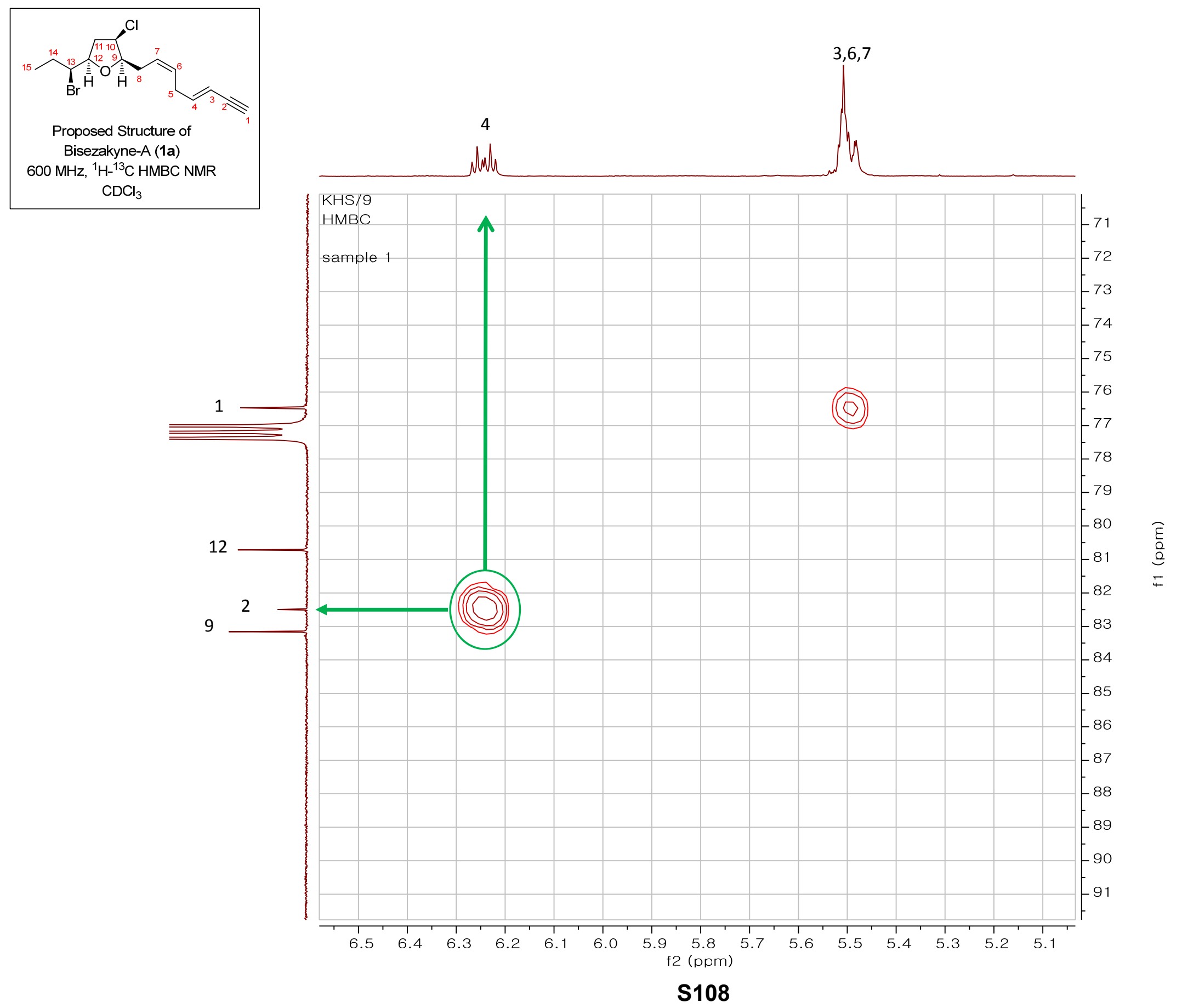




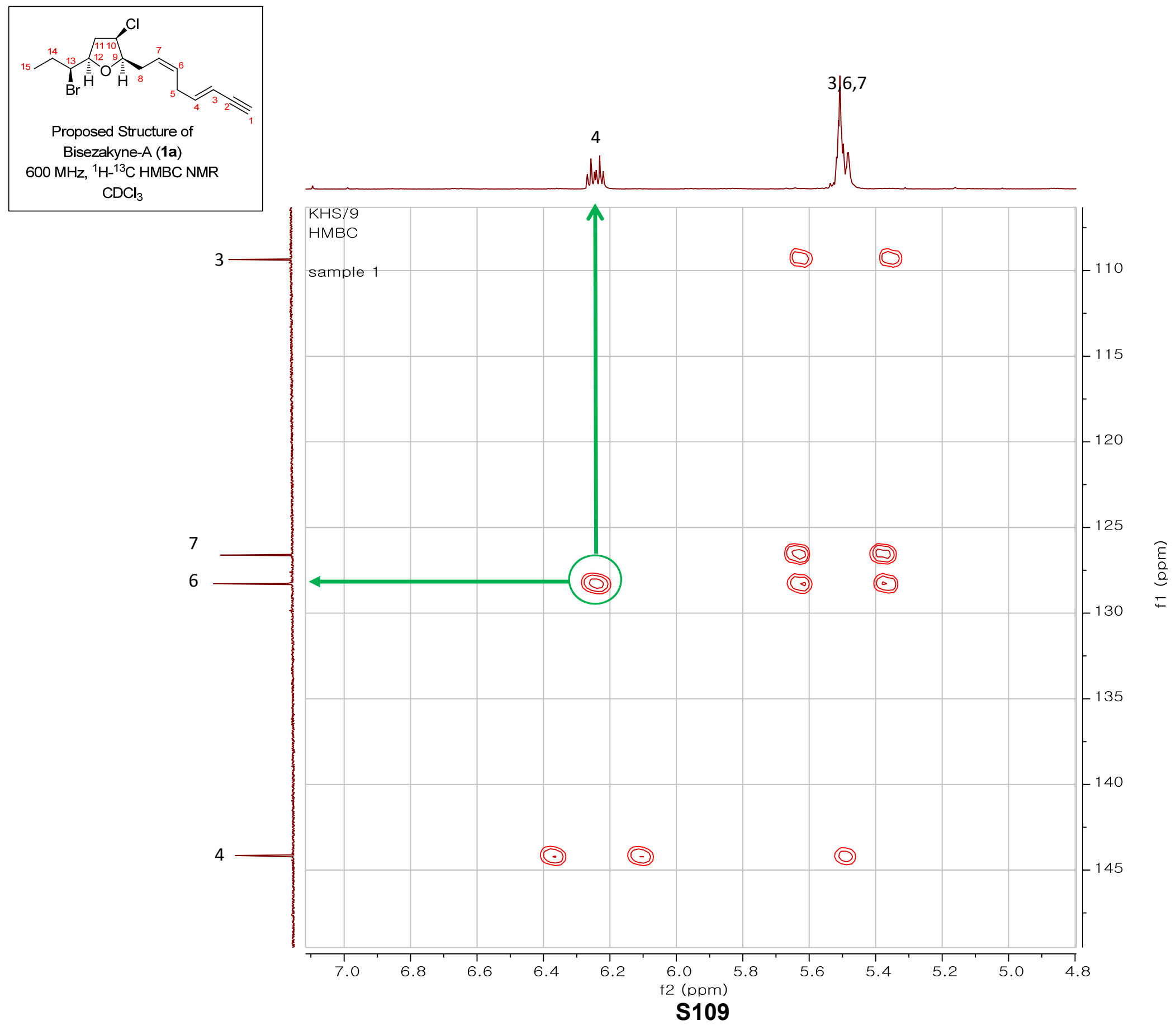




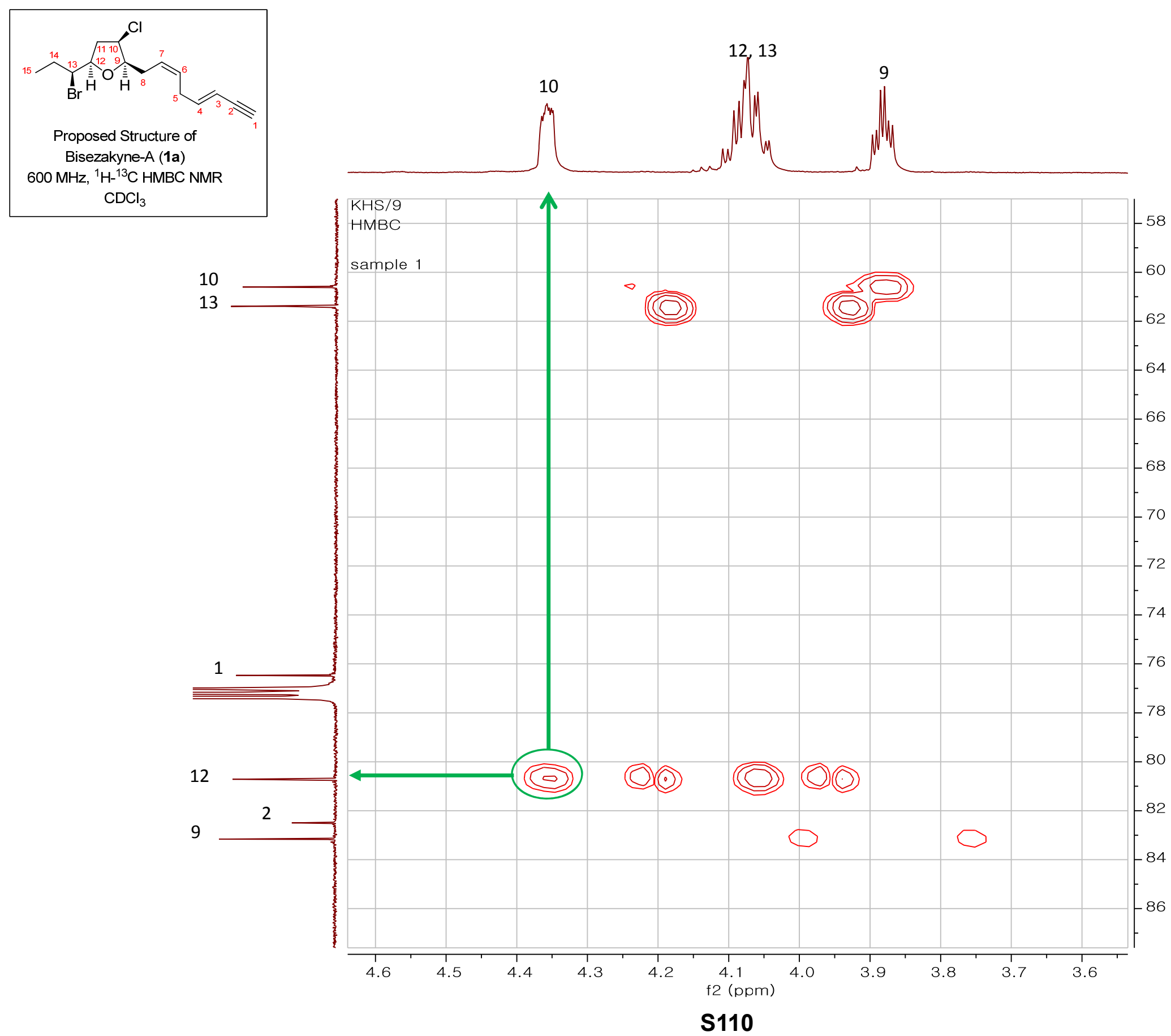




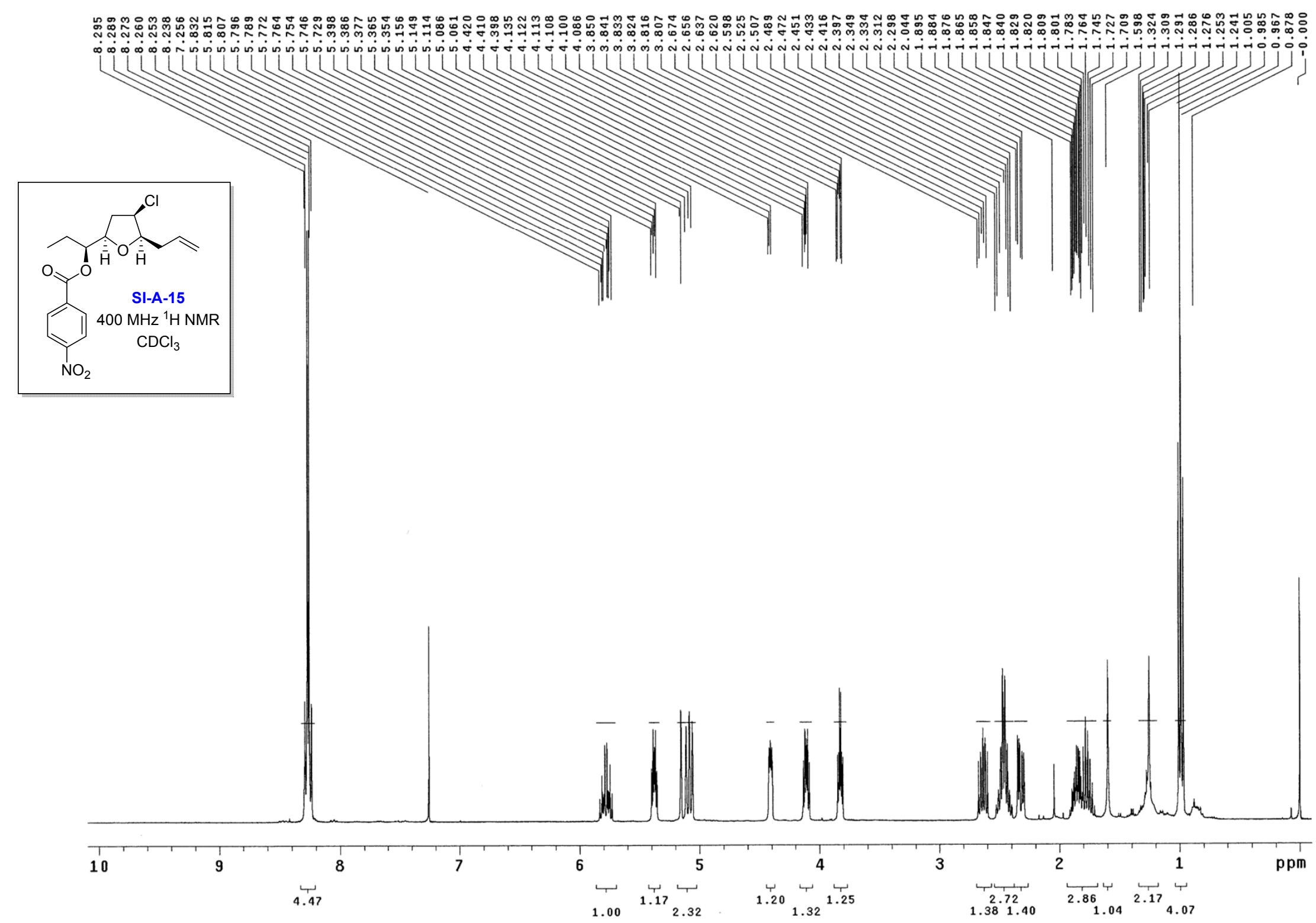




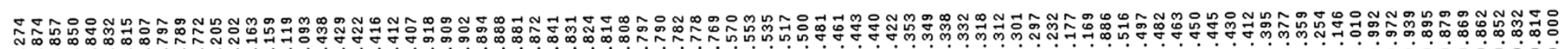

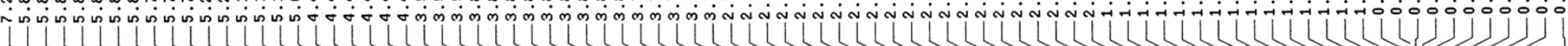

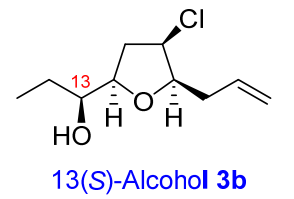

13(S)-Alcohol 3b $400 \mathrm{MHz}^{1} \mathrm{H} \mathrm{NMR}, \mathrm{CDCl}_{3}$
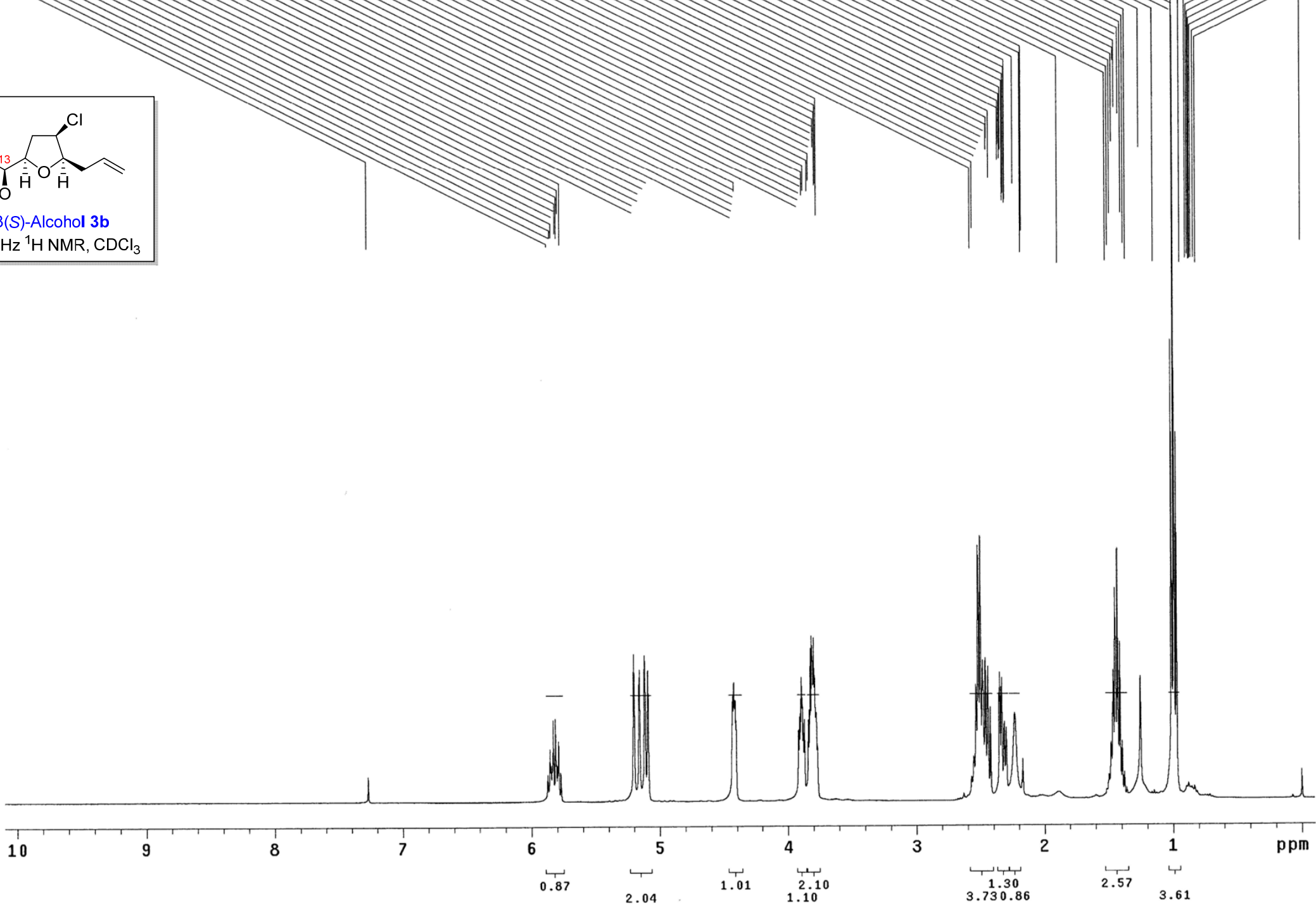

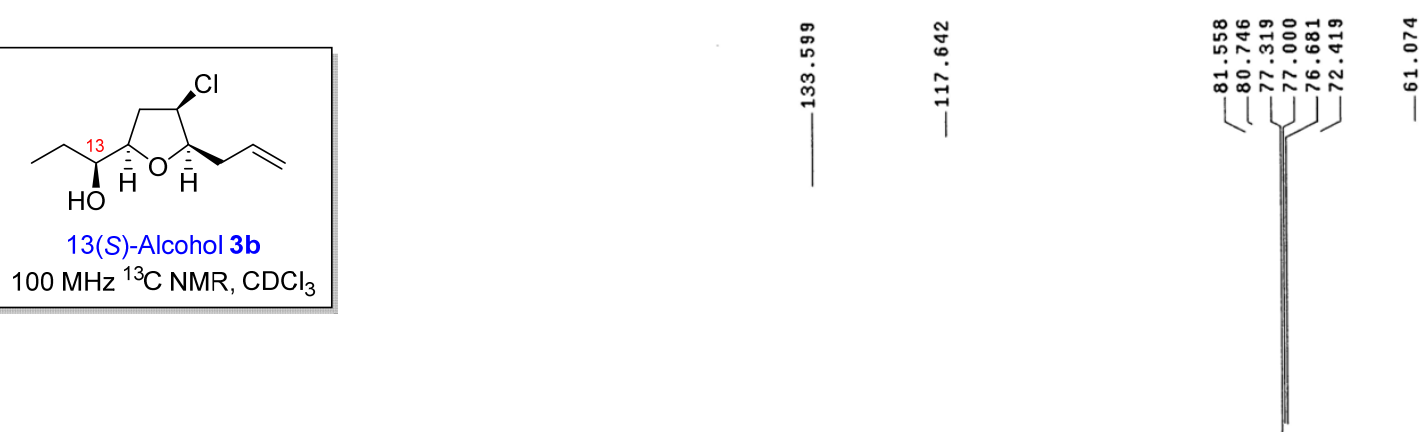

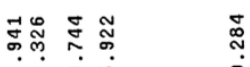

䟺 N

(S)-Alcohol 3b

$100 \mathrm{MHz}{ }^{13} \mathrm{C} \mathrm{NMR}, \mathrm{CDCl}_{3}$

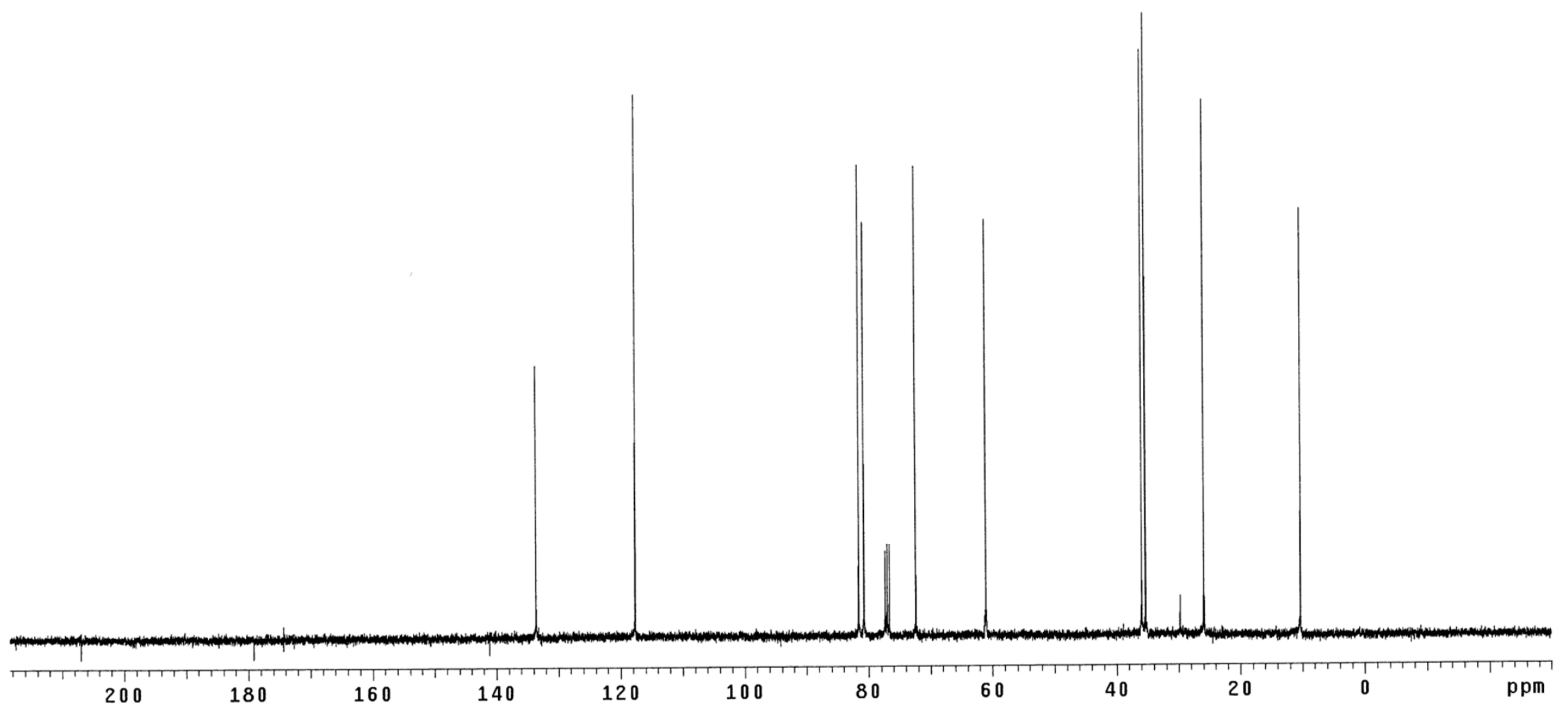




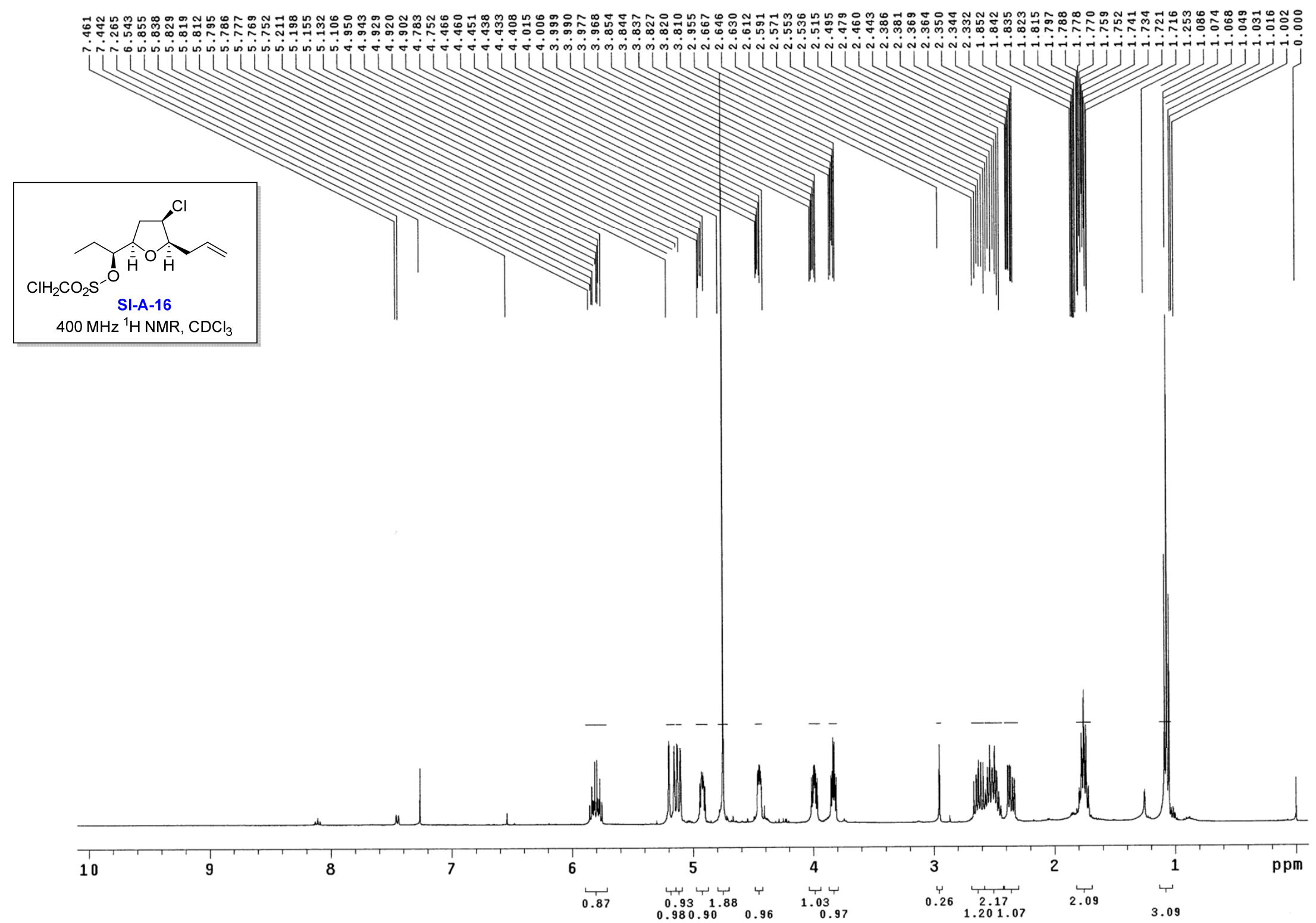

$\mathbf{S 1 1 4}$ 


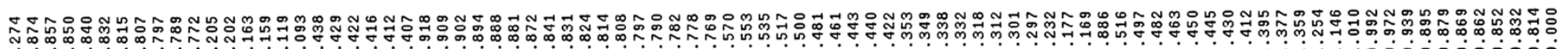
ن́rín
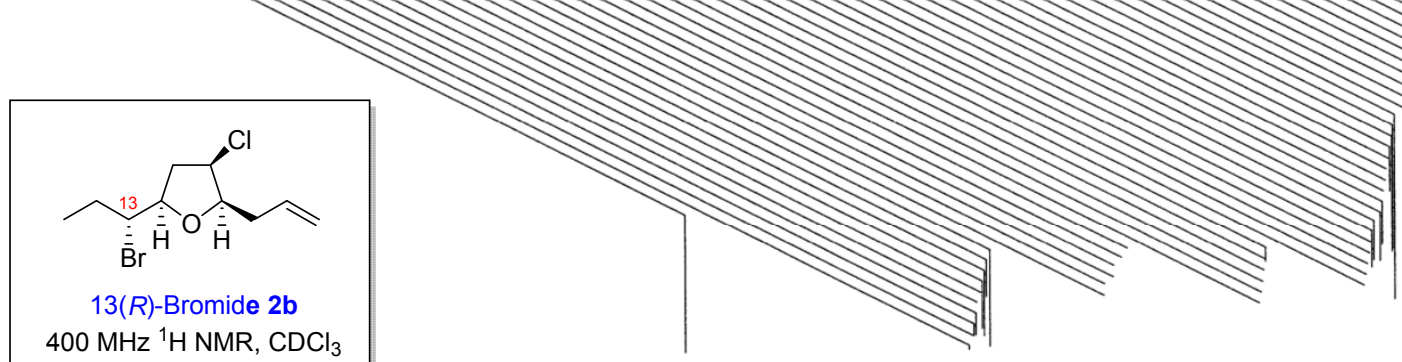

$400 \mathrm{MHz}{ }^{1} \mathrm{H}$ NMR, CDCl
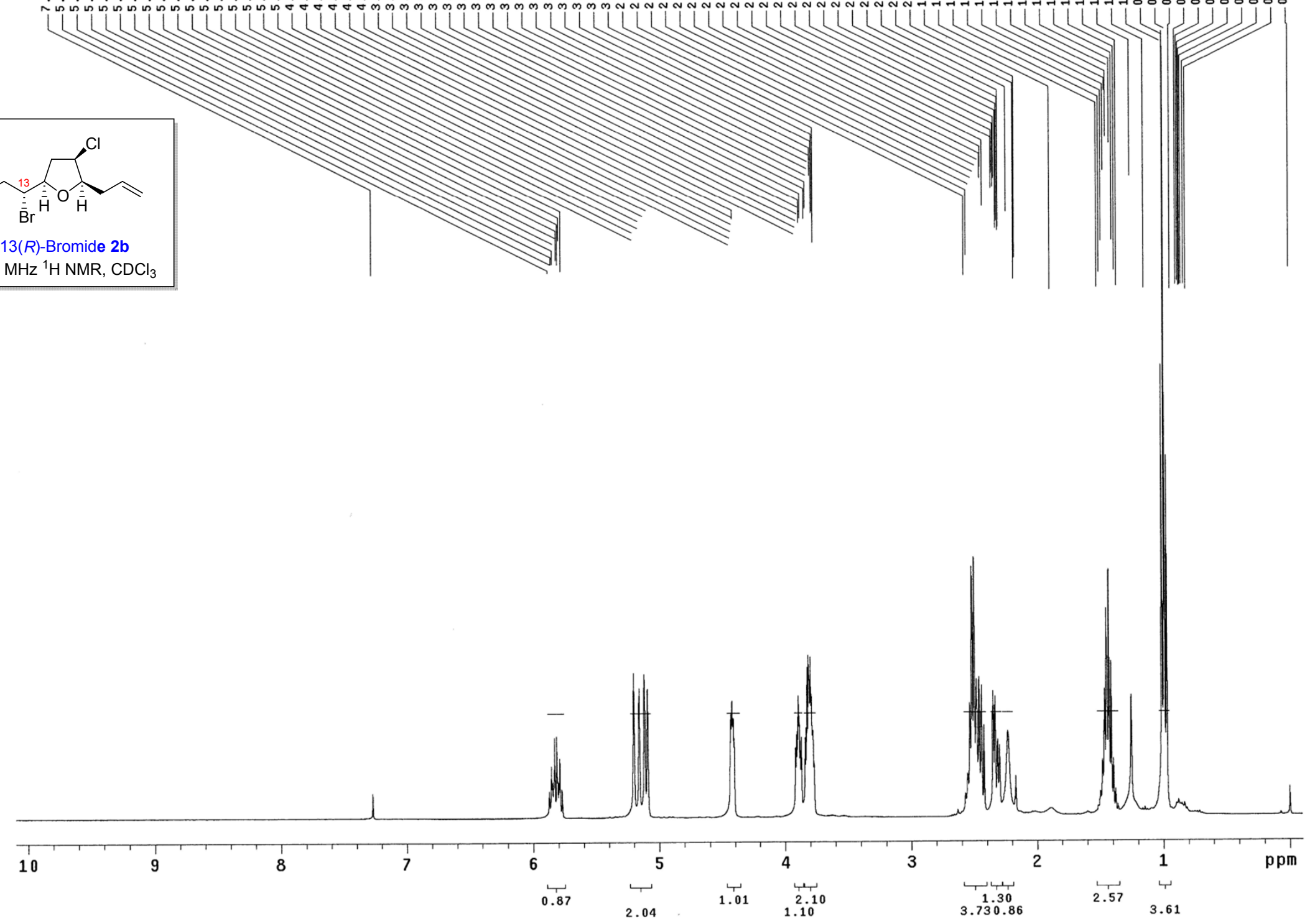

S115 
$3(R)$-Bromide 2b

$175 \mathrm{MHz}^{13} \mathrm{C} \mathrm{NMR}, \mathrm{CDCl}_{3}$

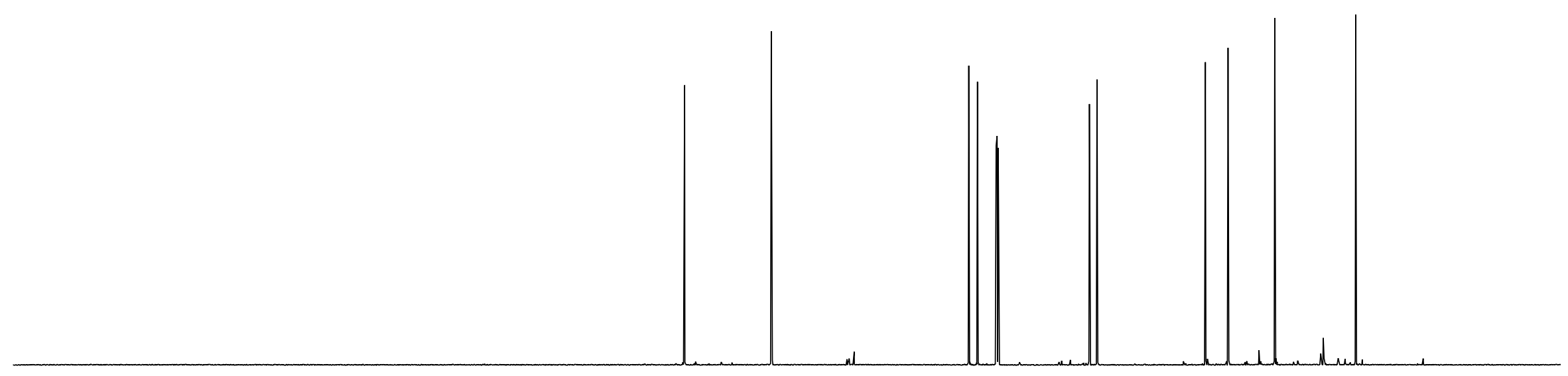

$25024023022021020019018017016015014013012011010090 \quad 8070 \quad 6050 \quad 4030 \quad 2010 \quad 0 \quad-10-20$ f1 (ppm) 


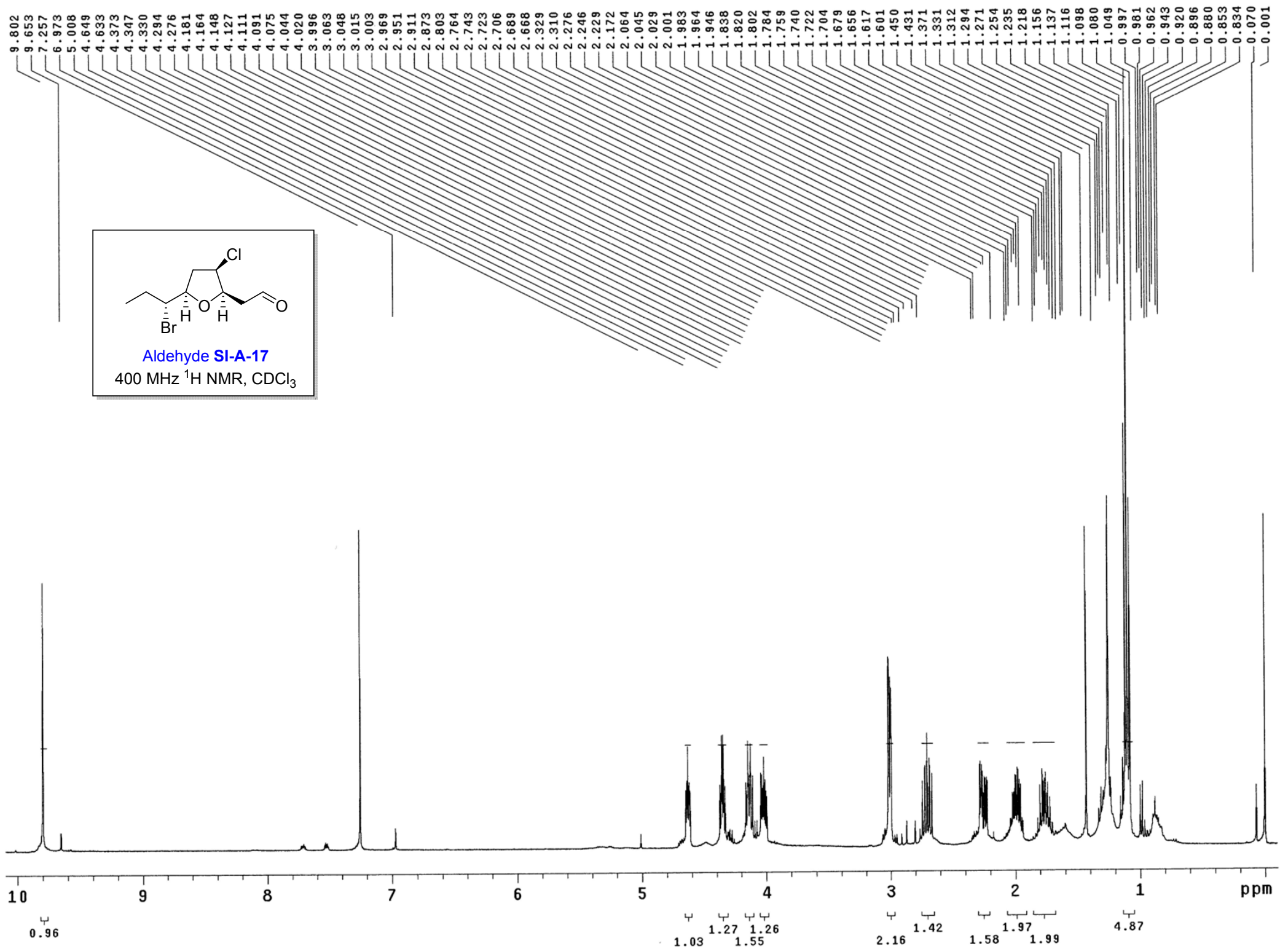




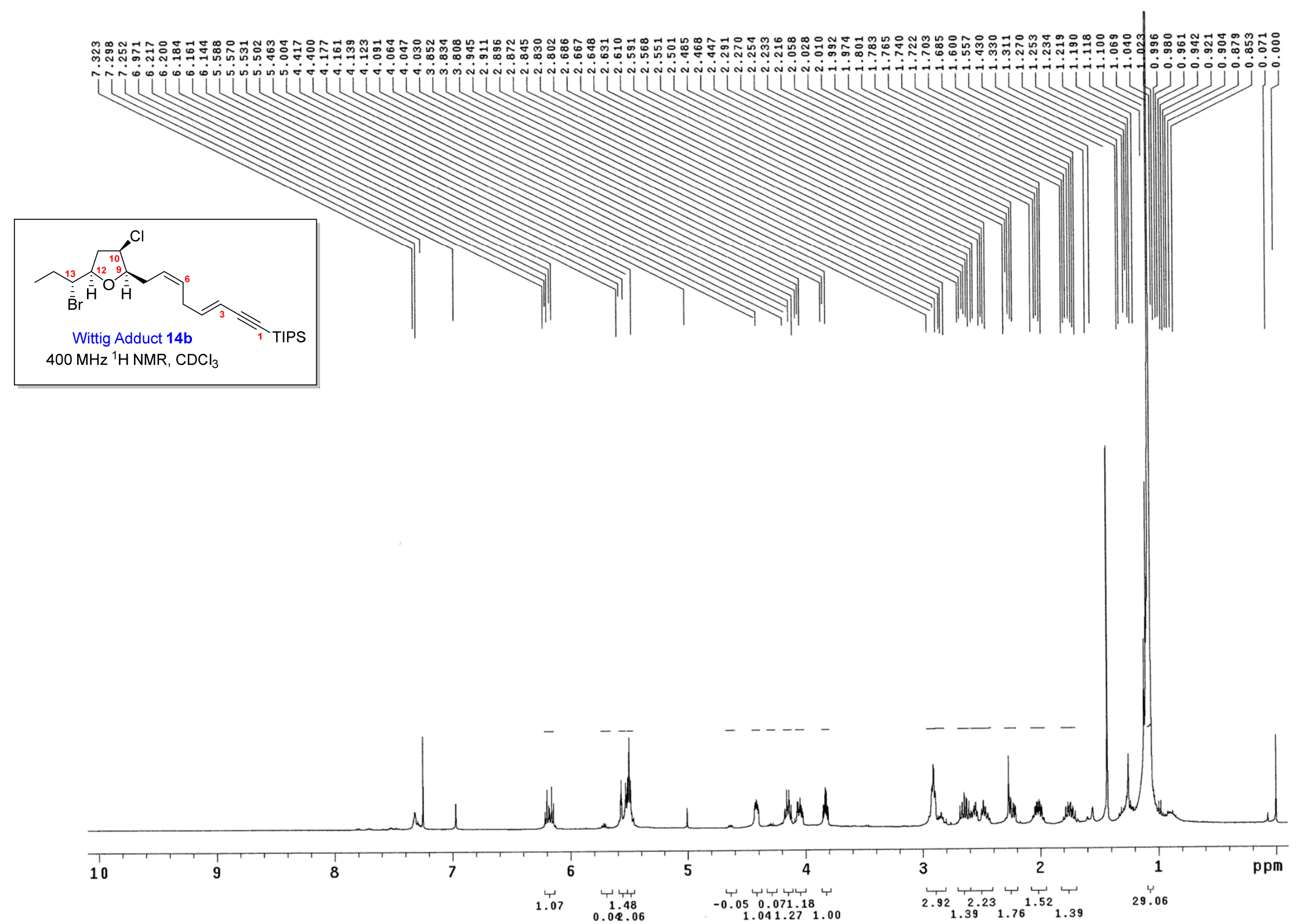




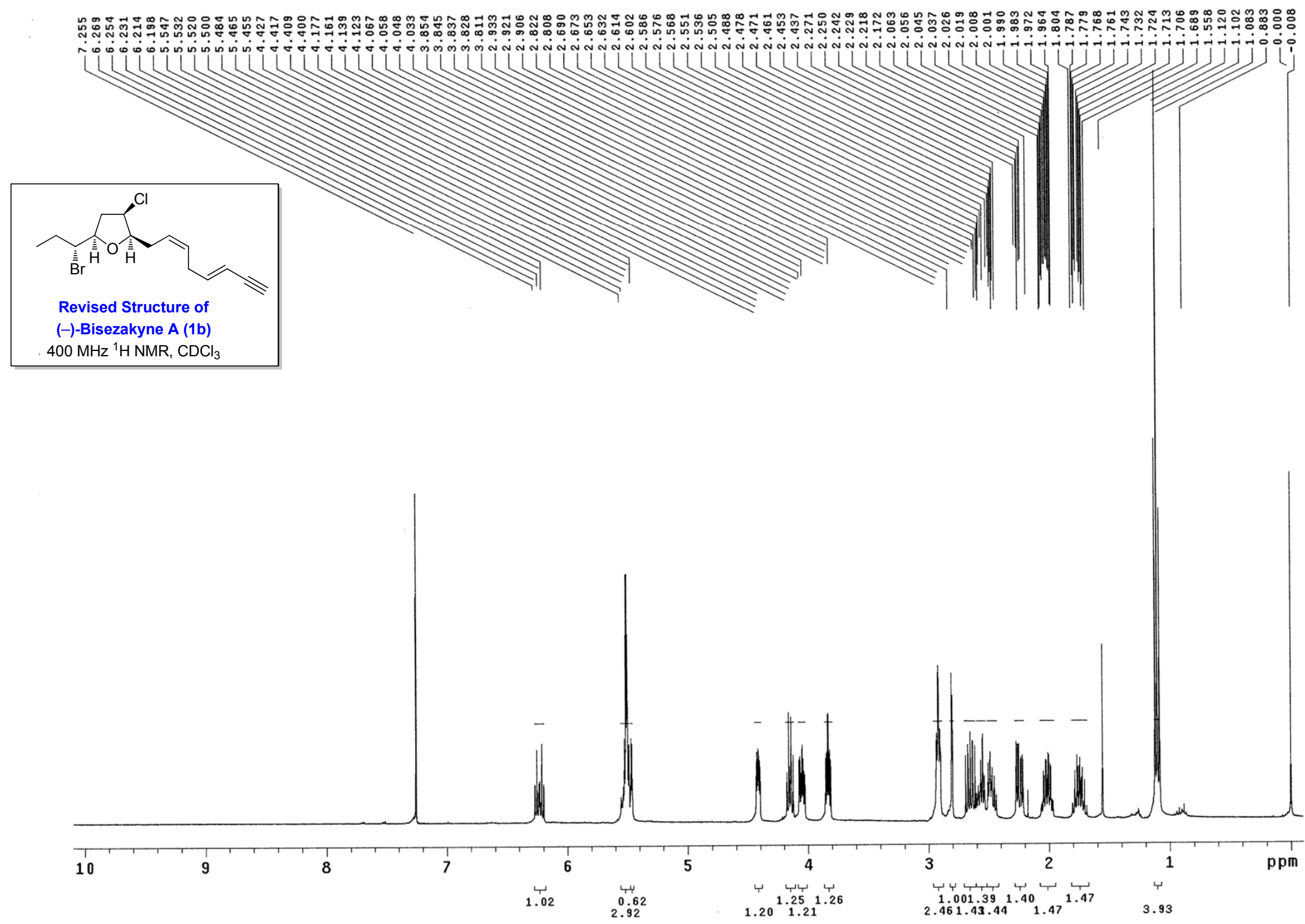



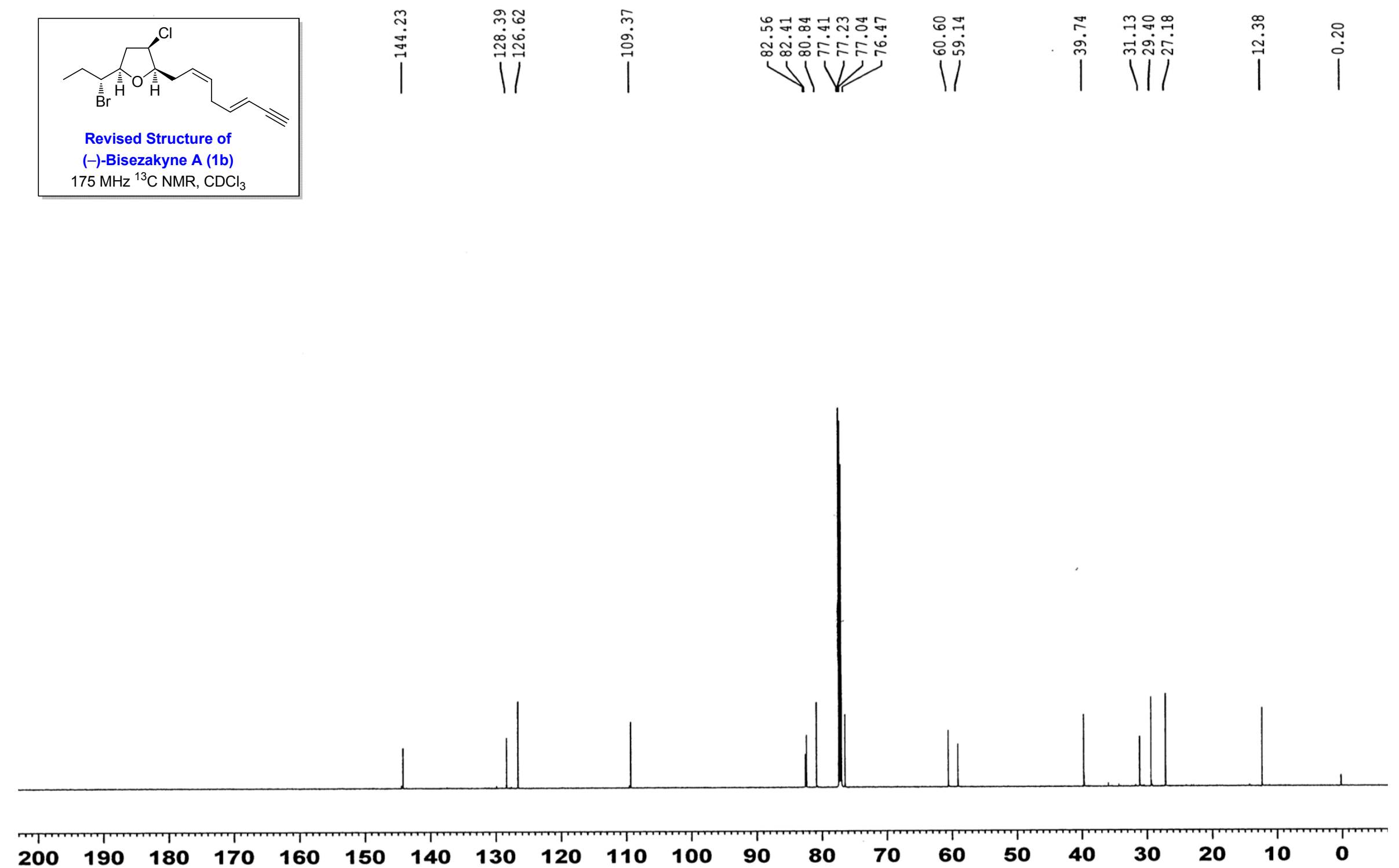


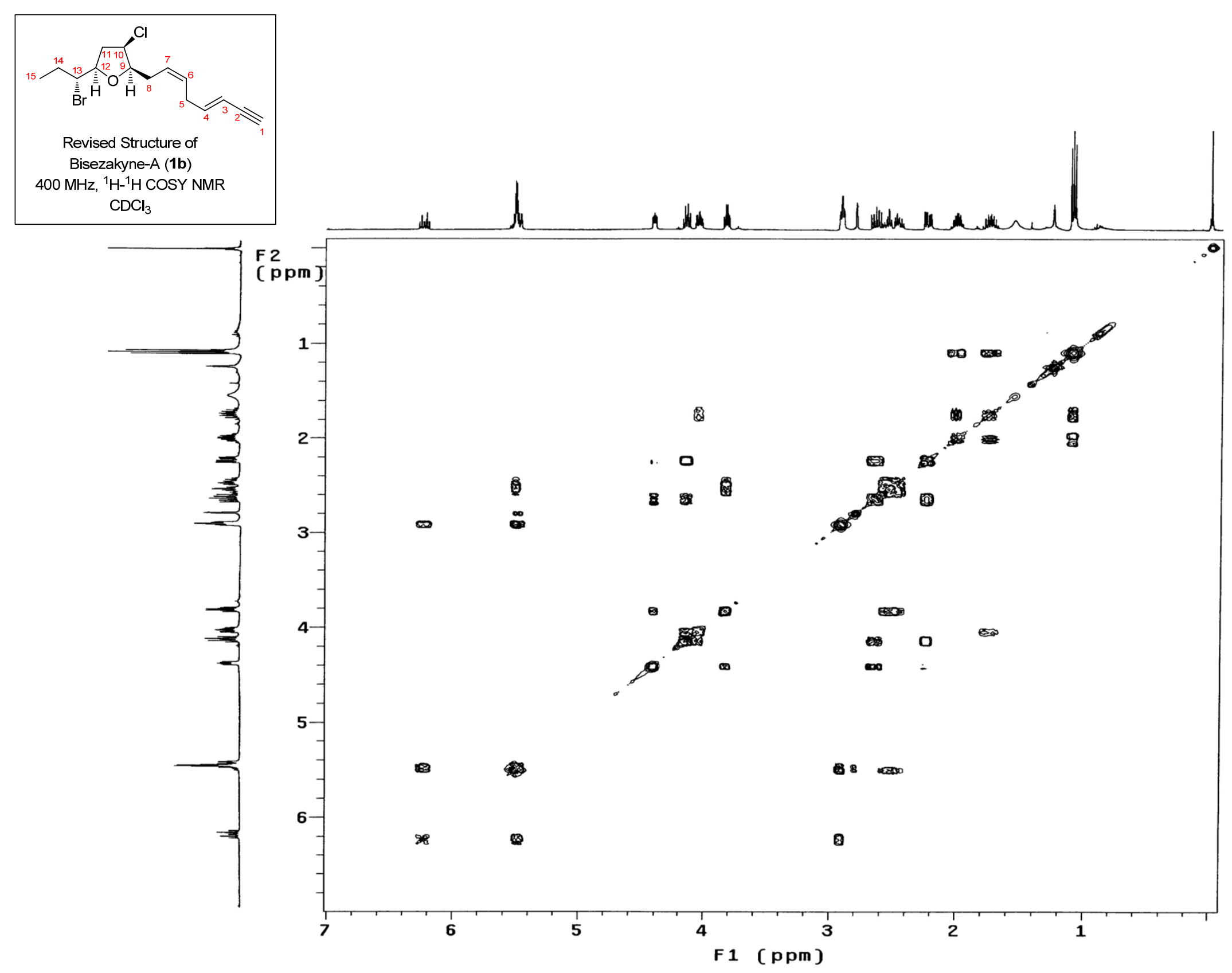




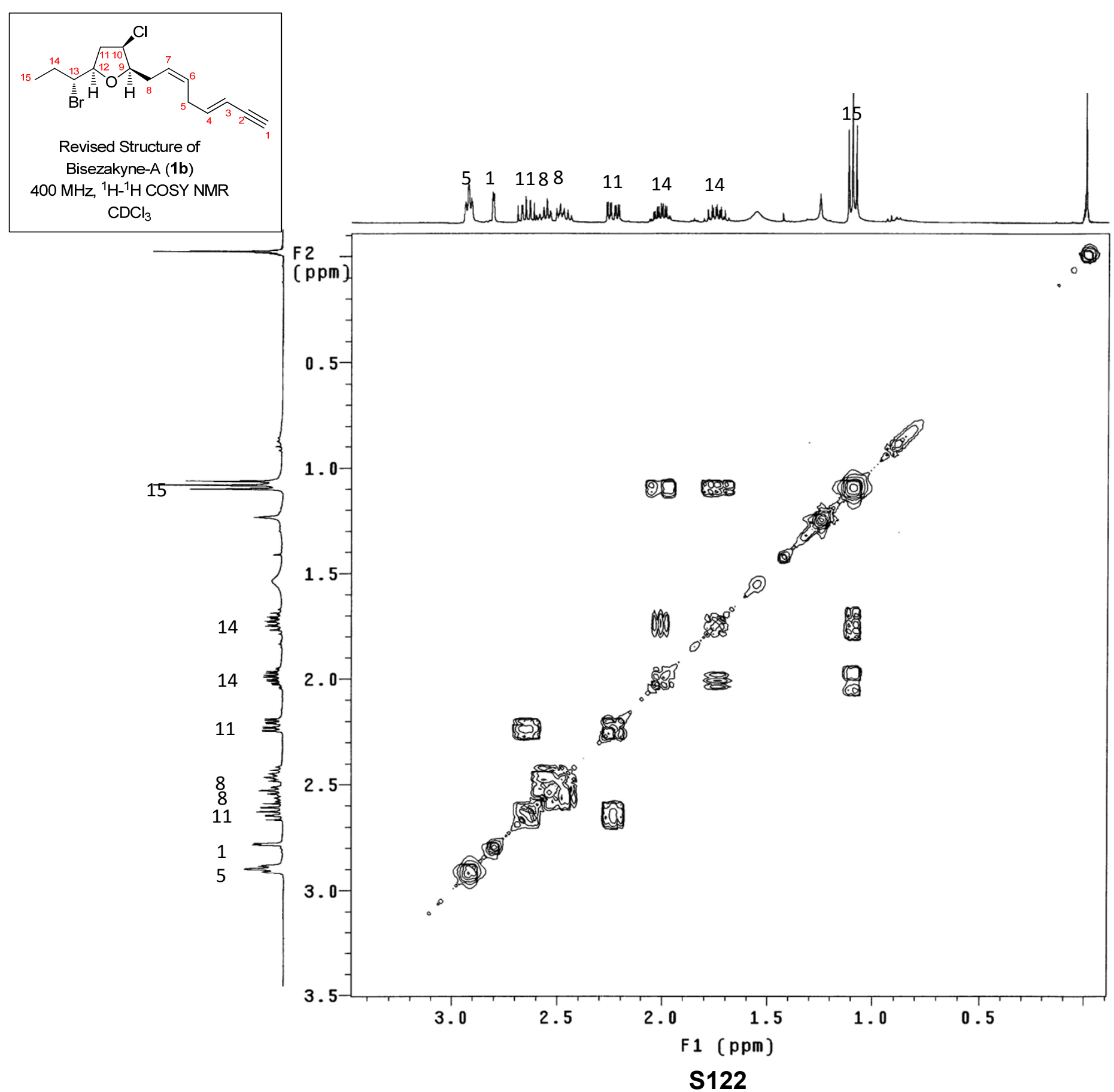




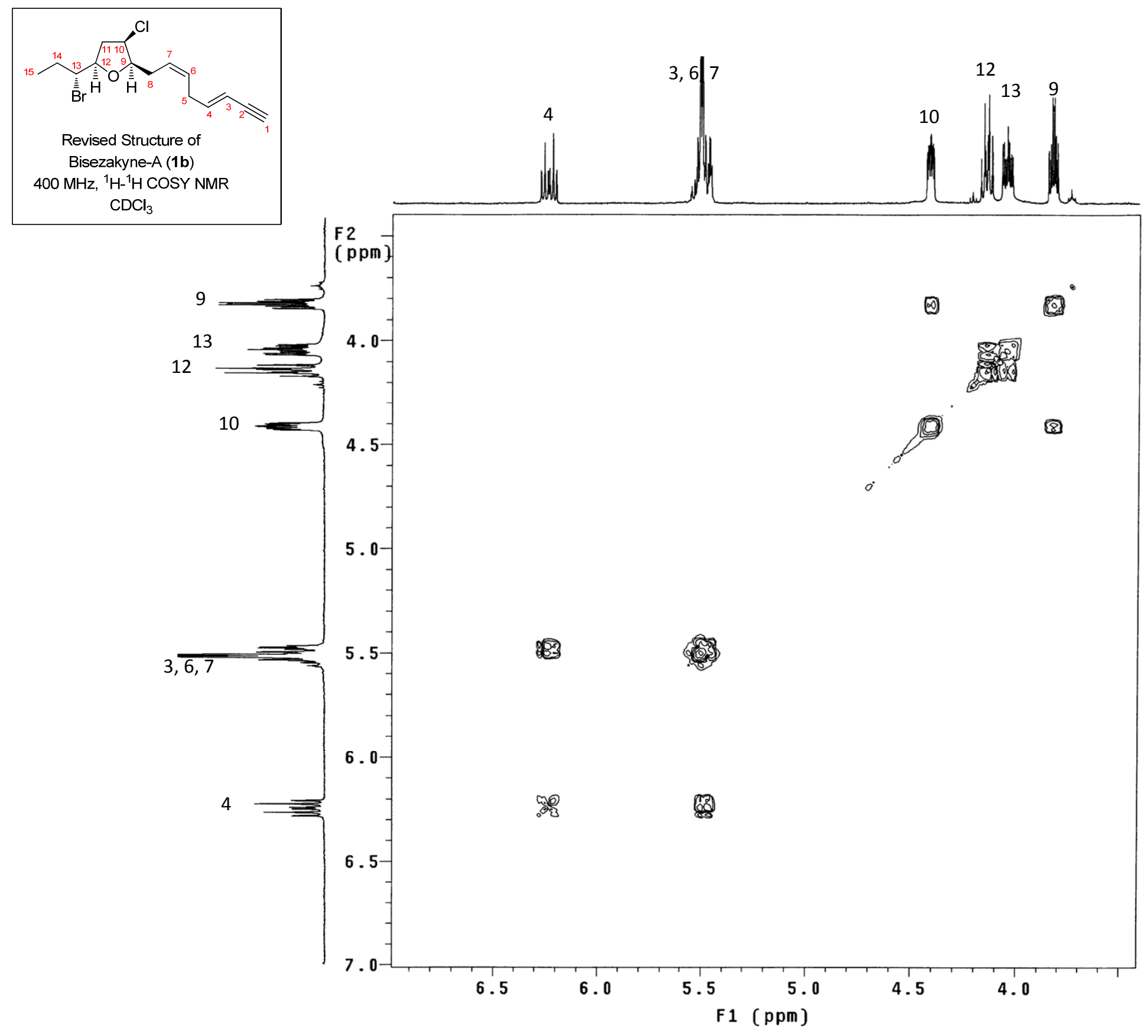

S123 


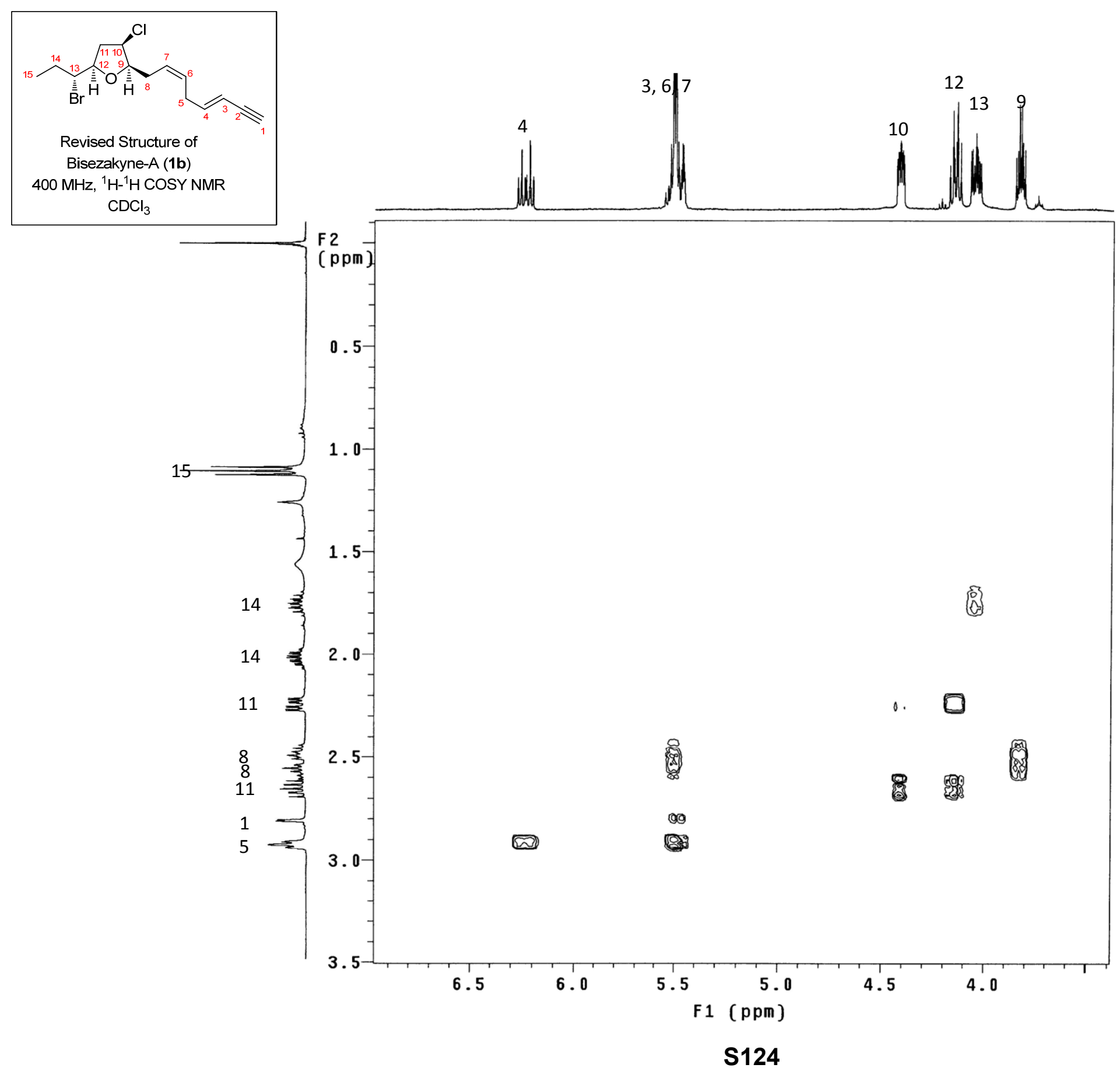




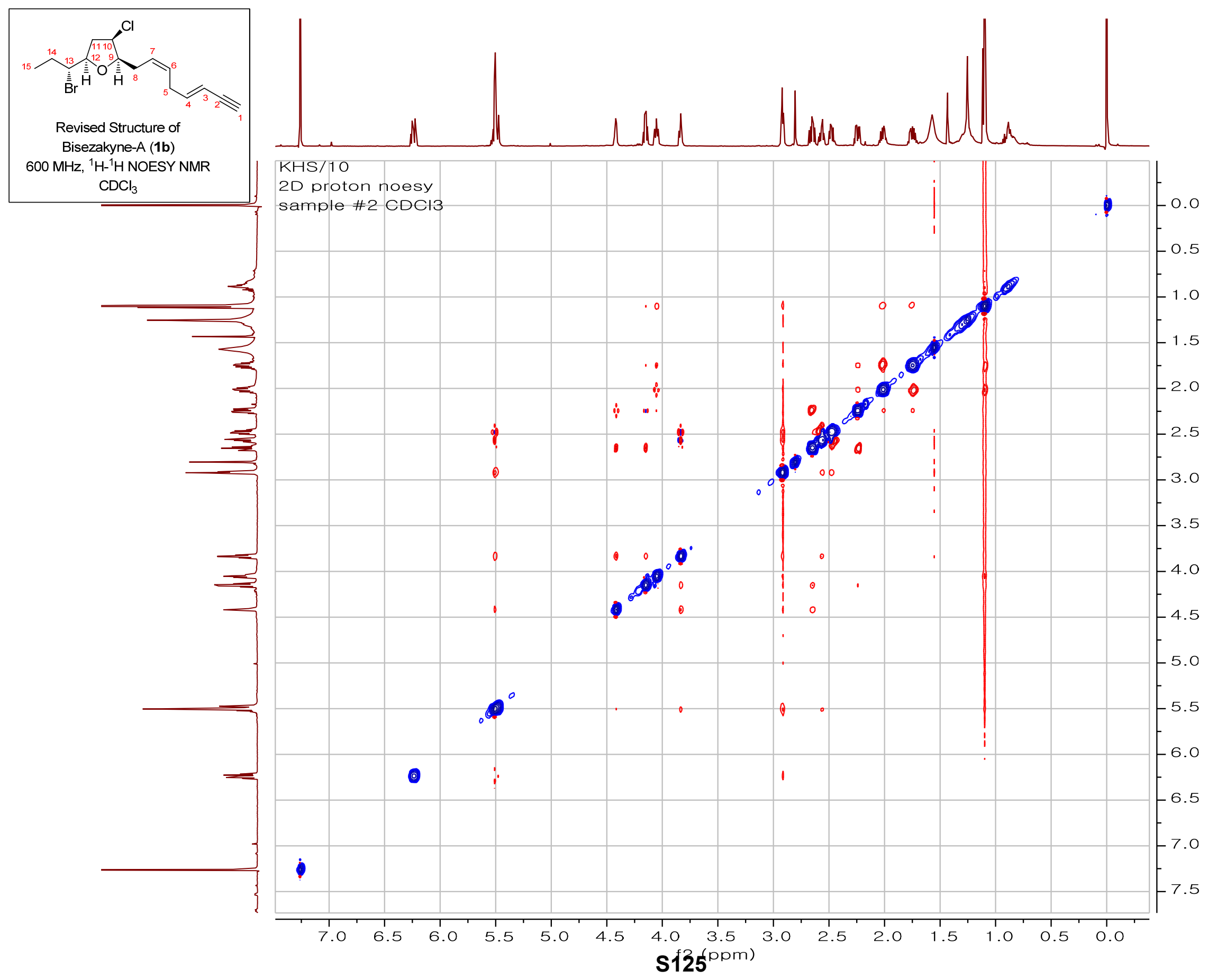




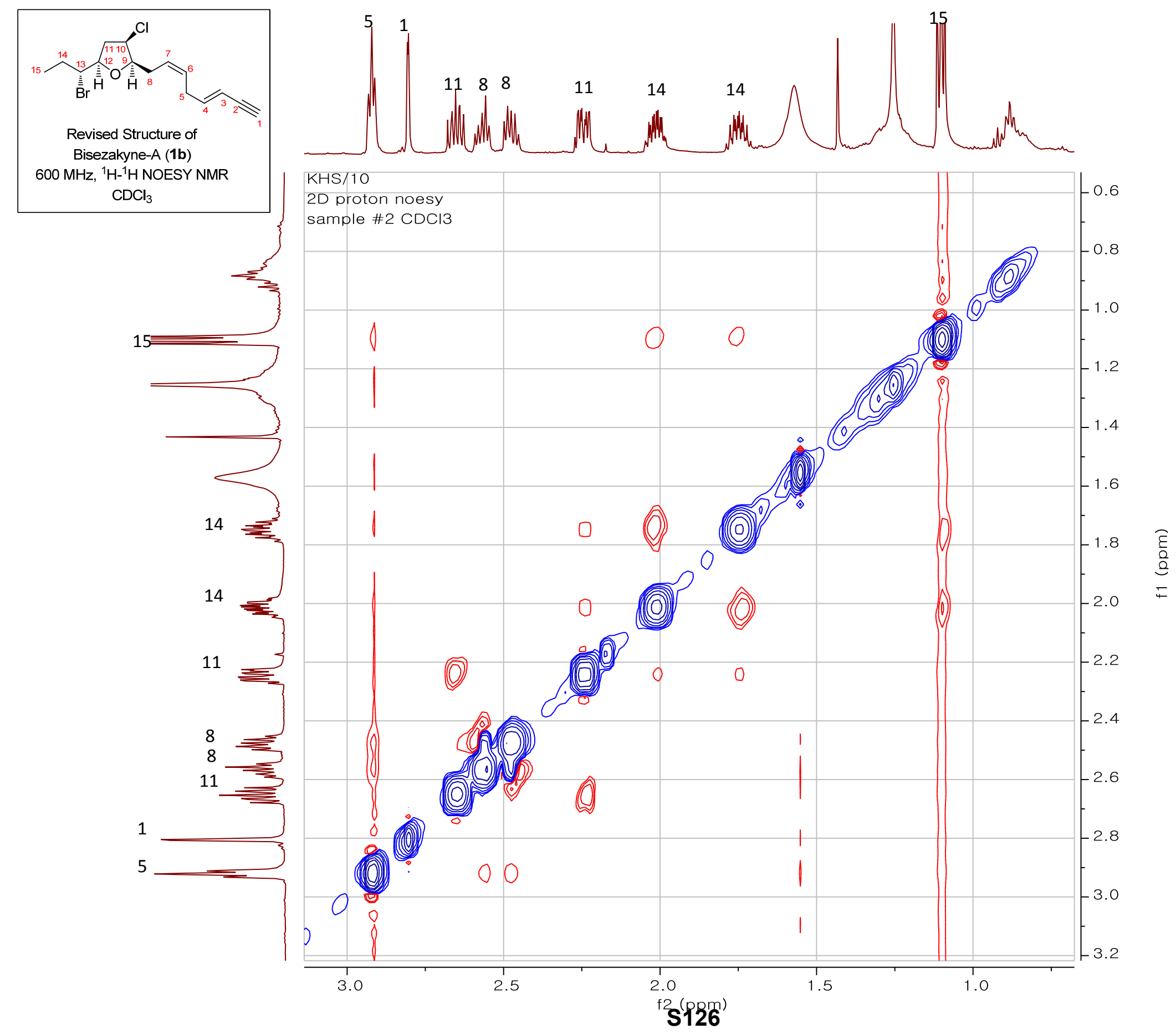




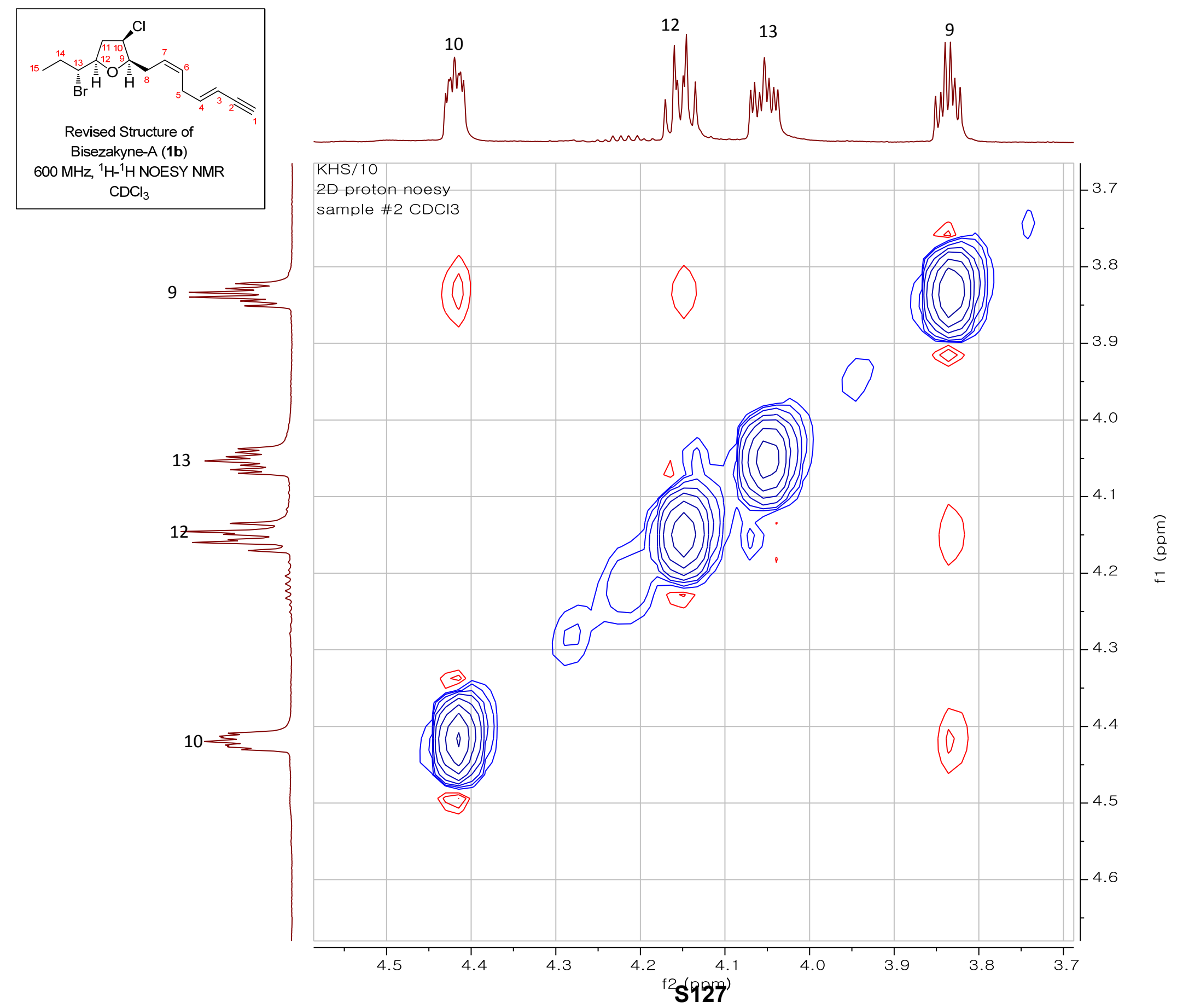




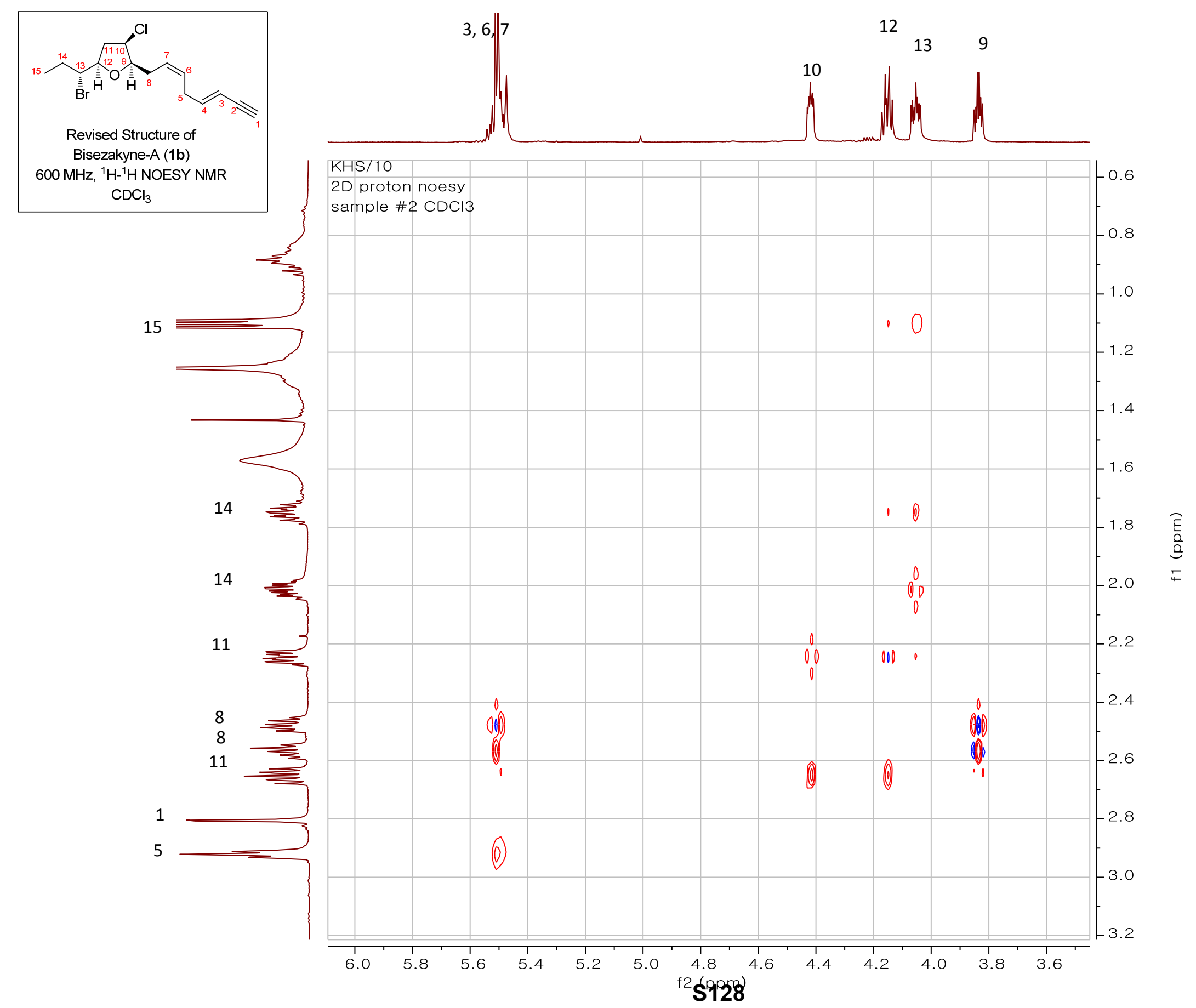



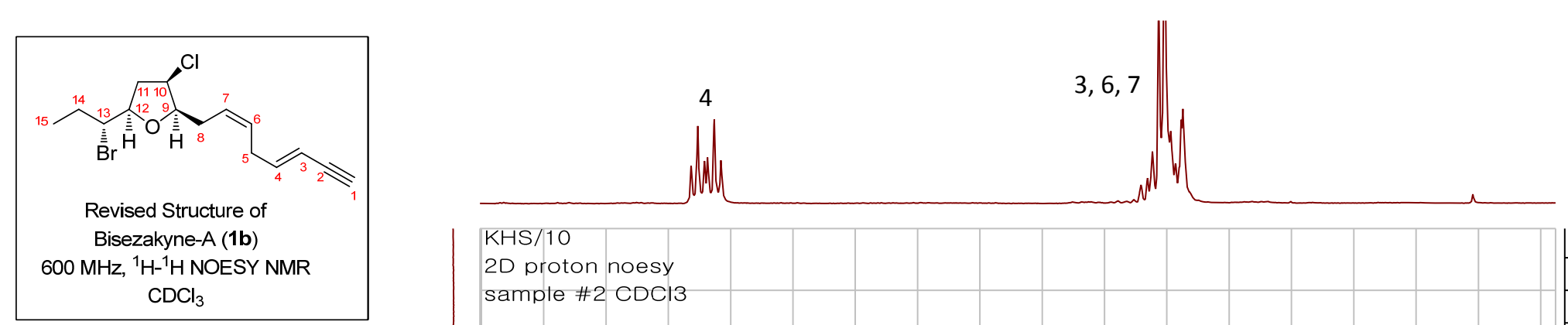

2D proton noesy

sample \#2 CDCI3

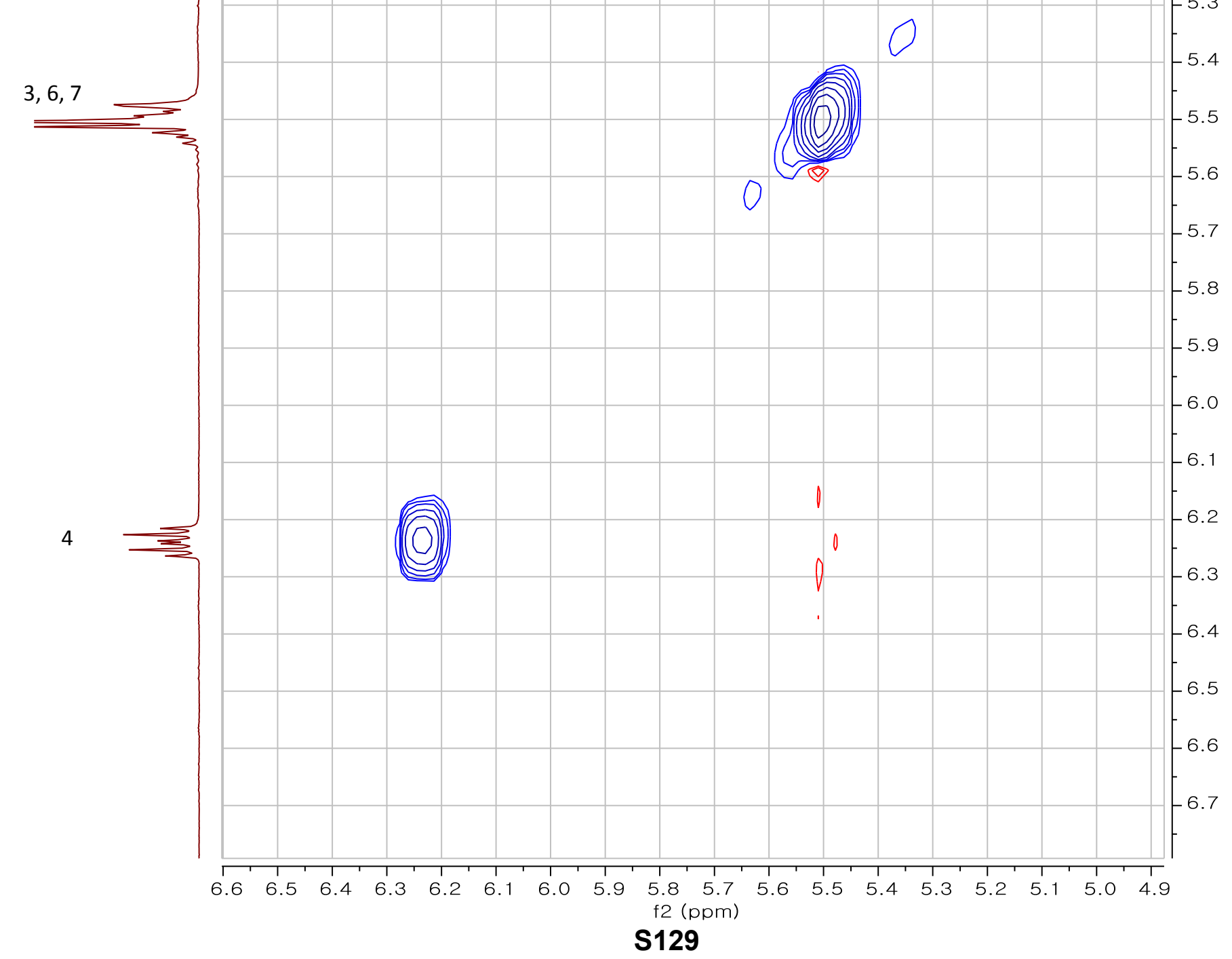

$\bar{\varepsilon}$
$\bar{a}$
$\bar{c}$ 


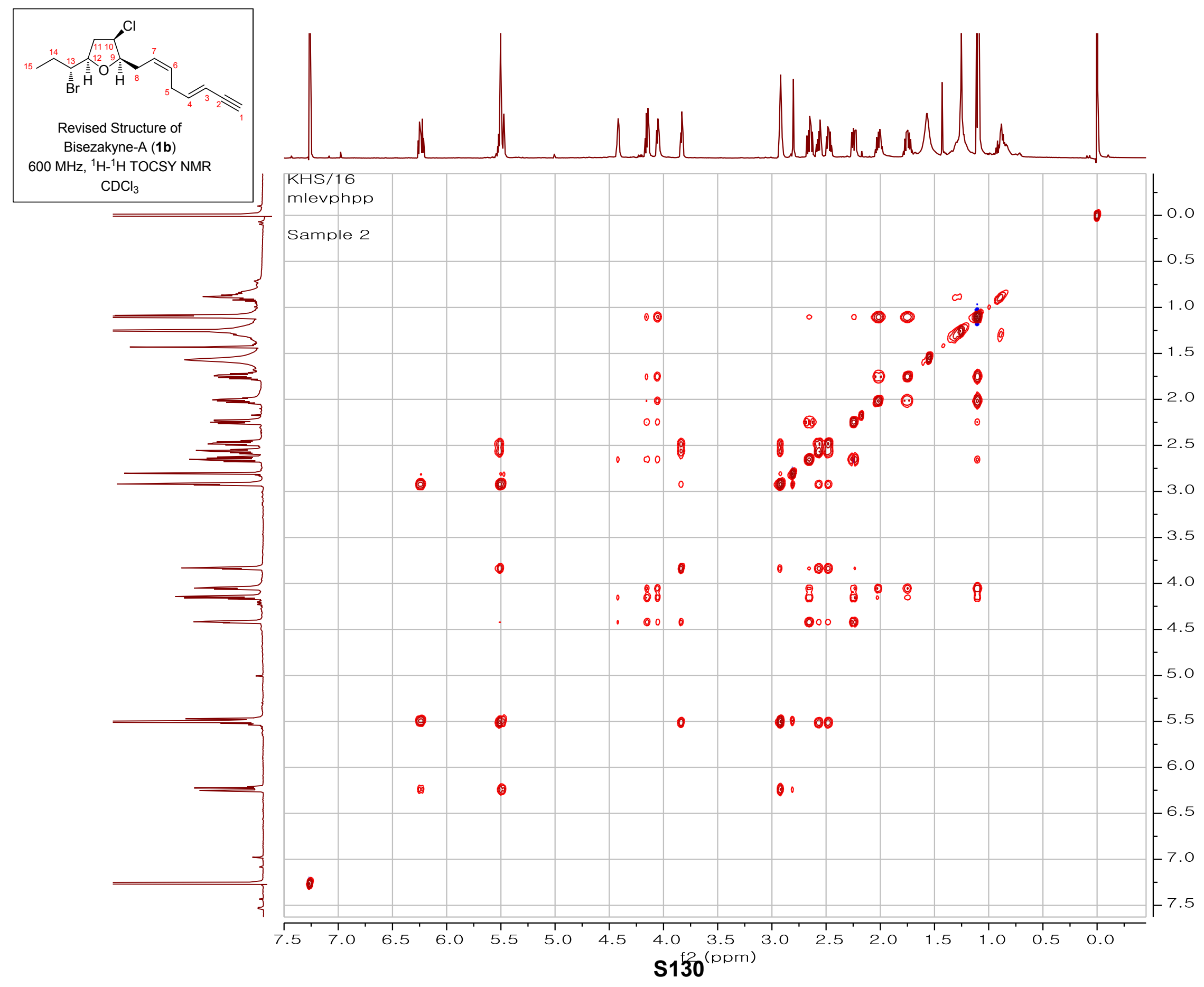




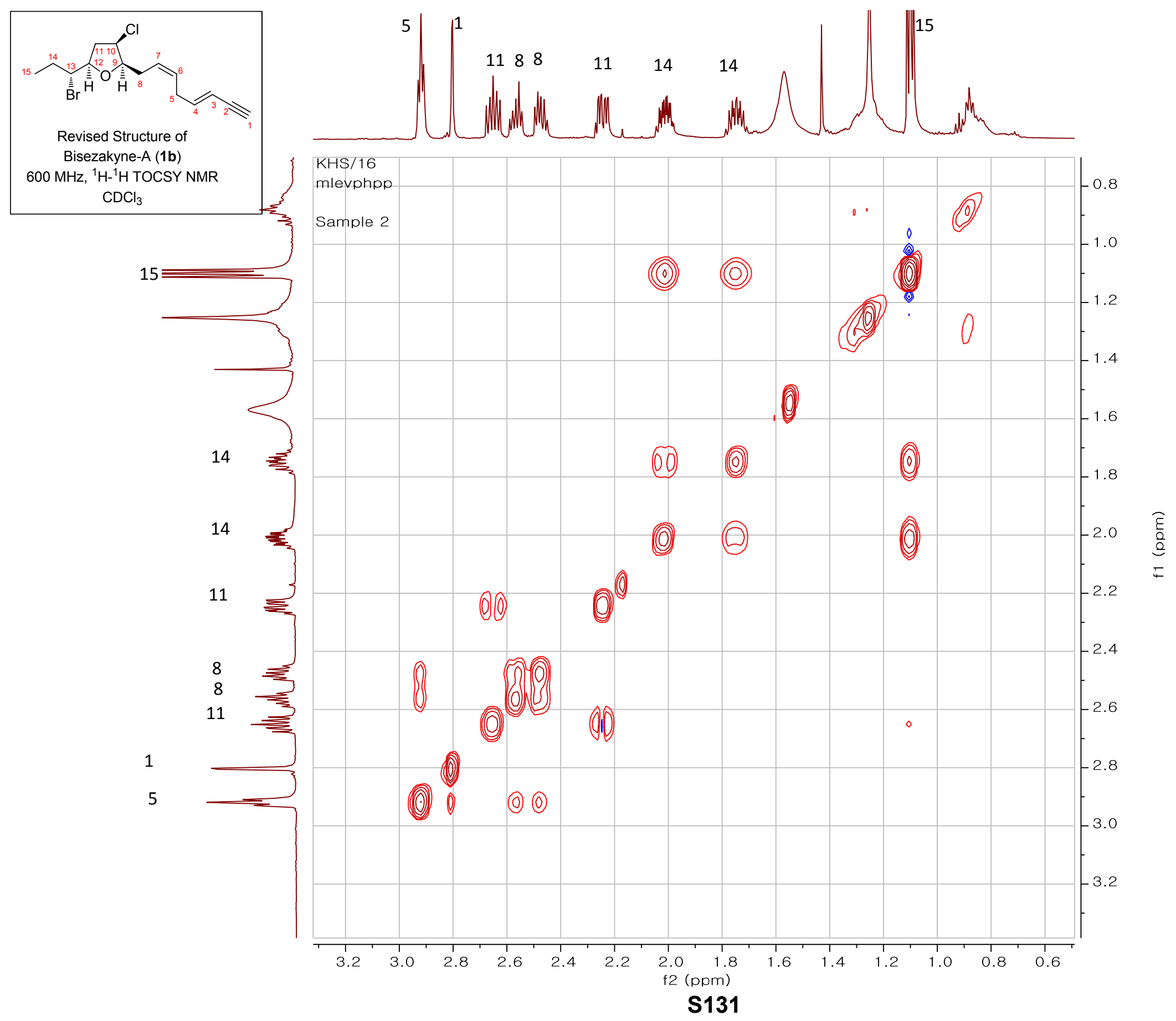




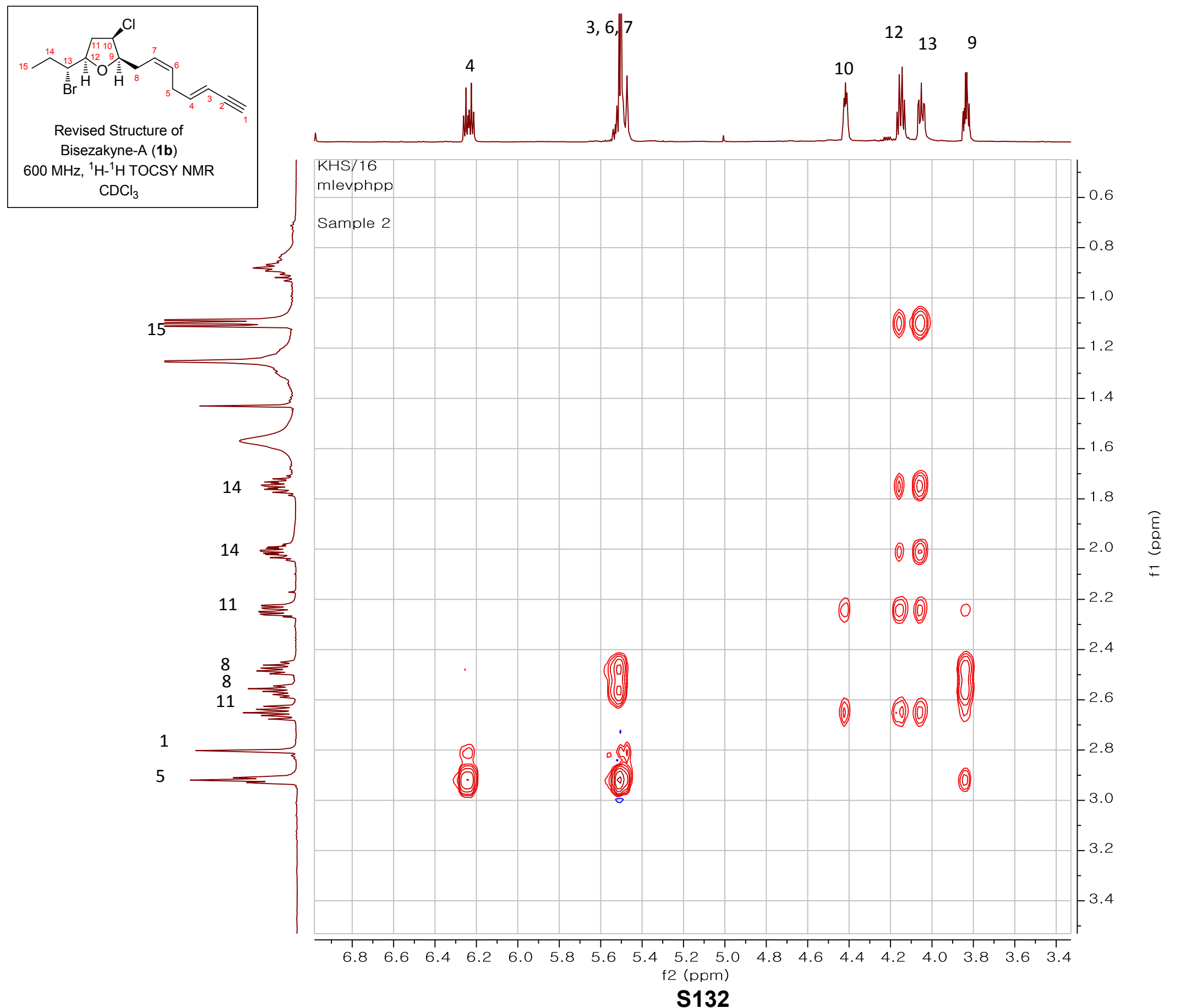




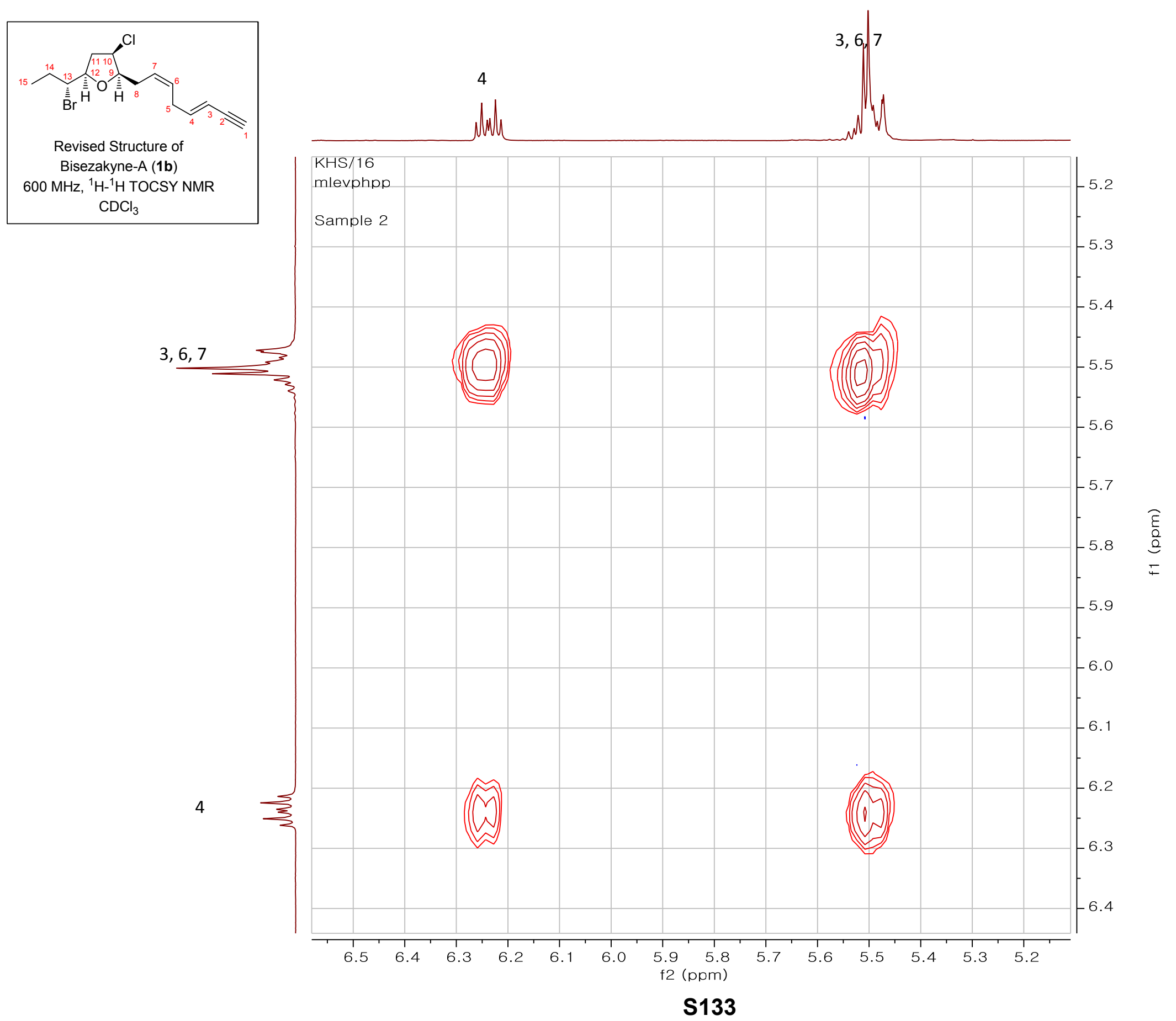




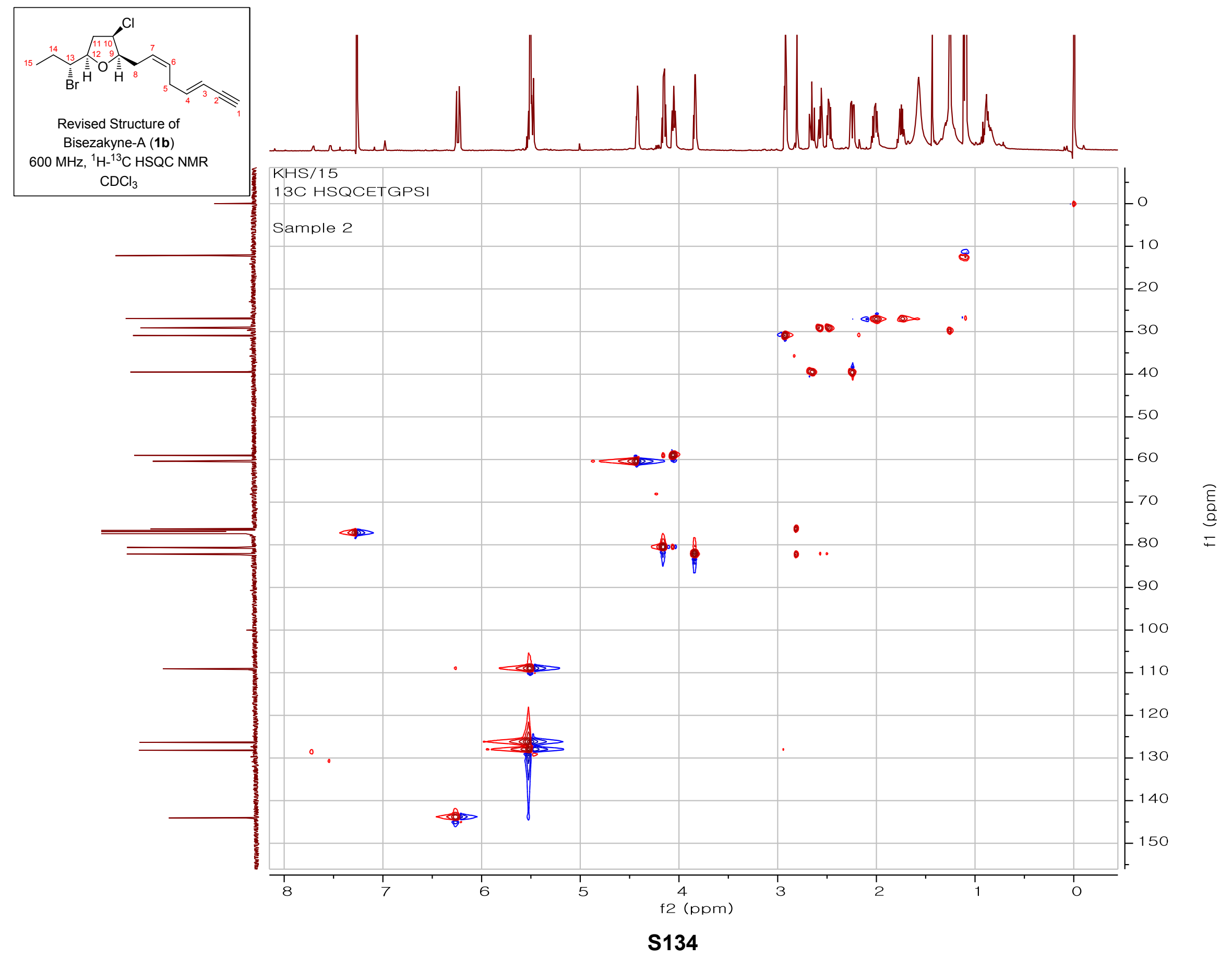




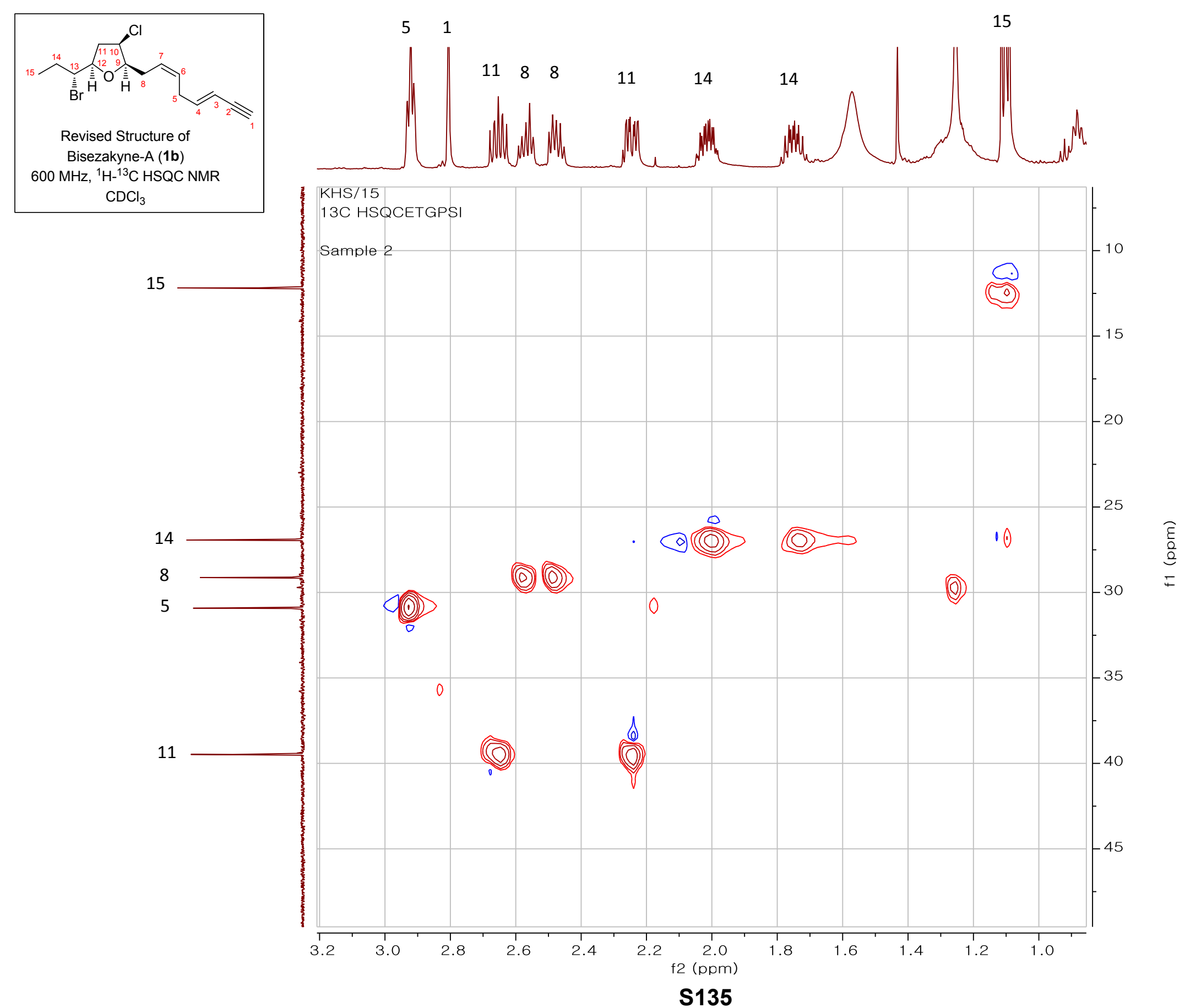




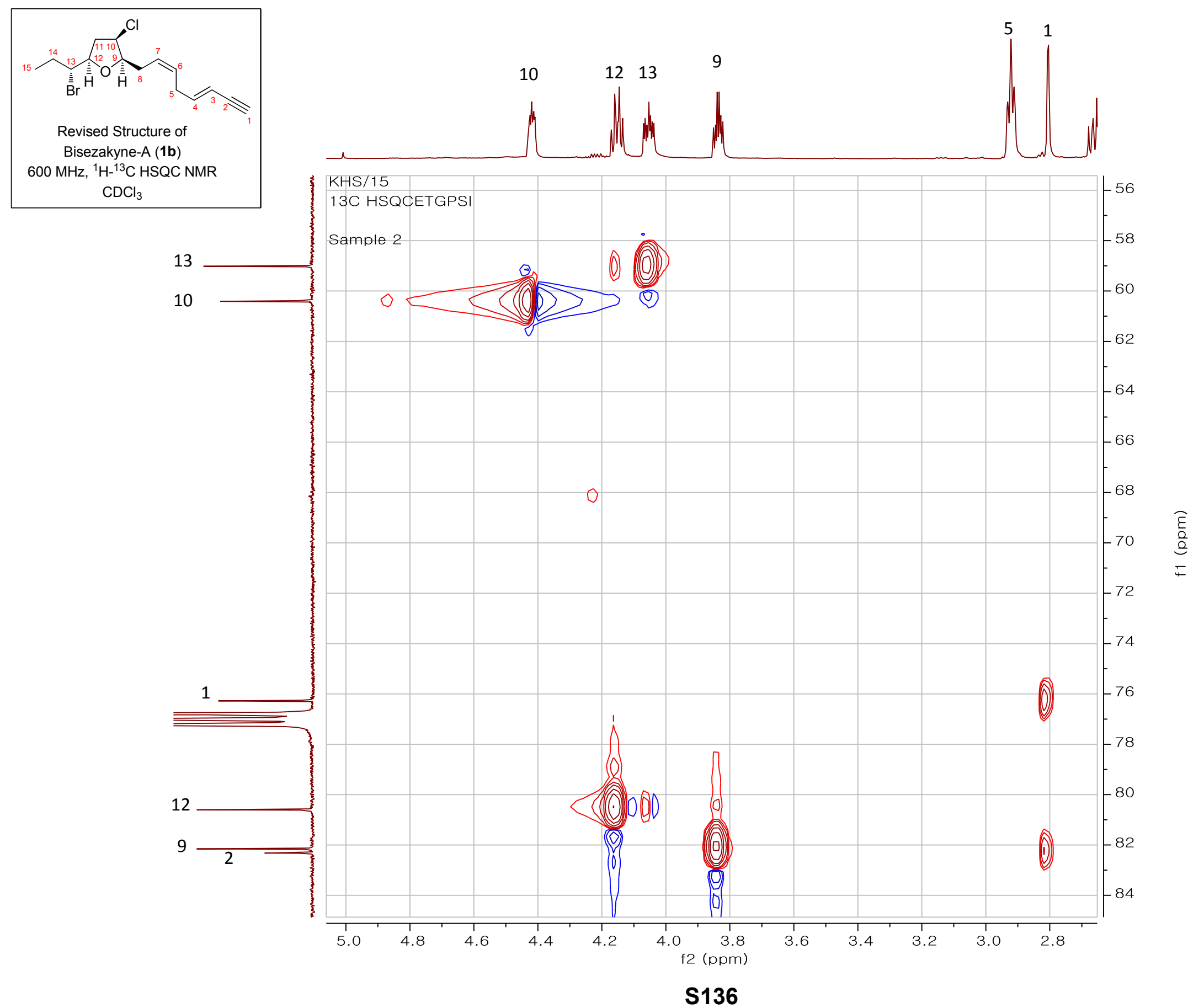



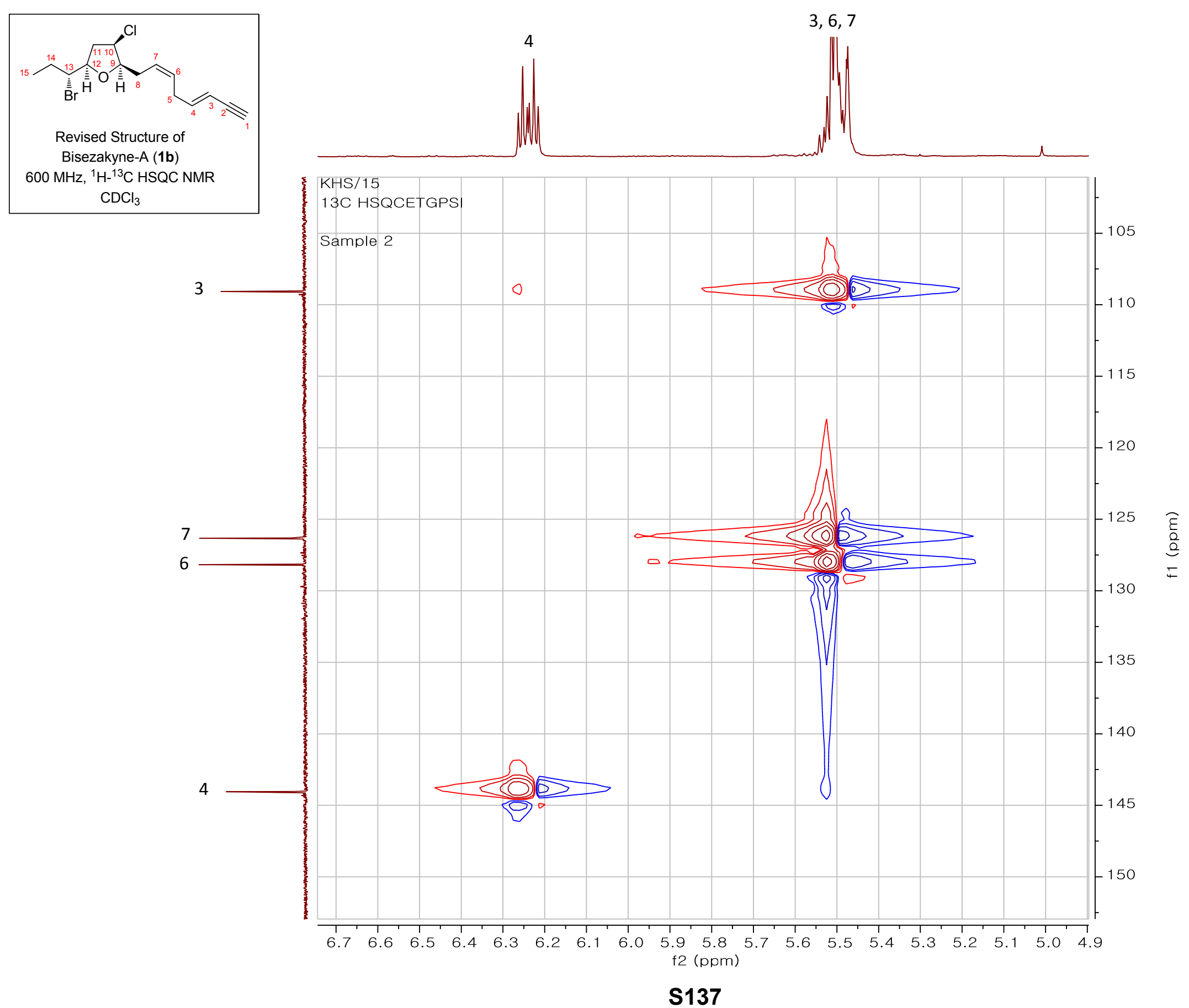


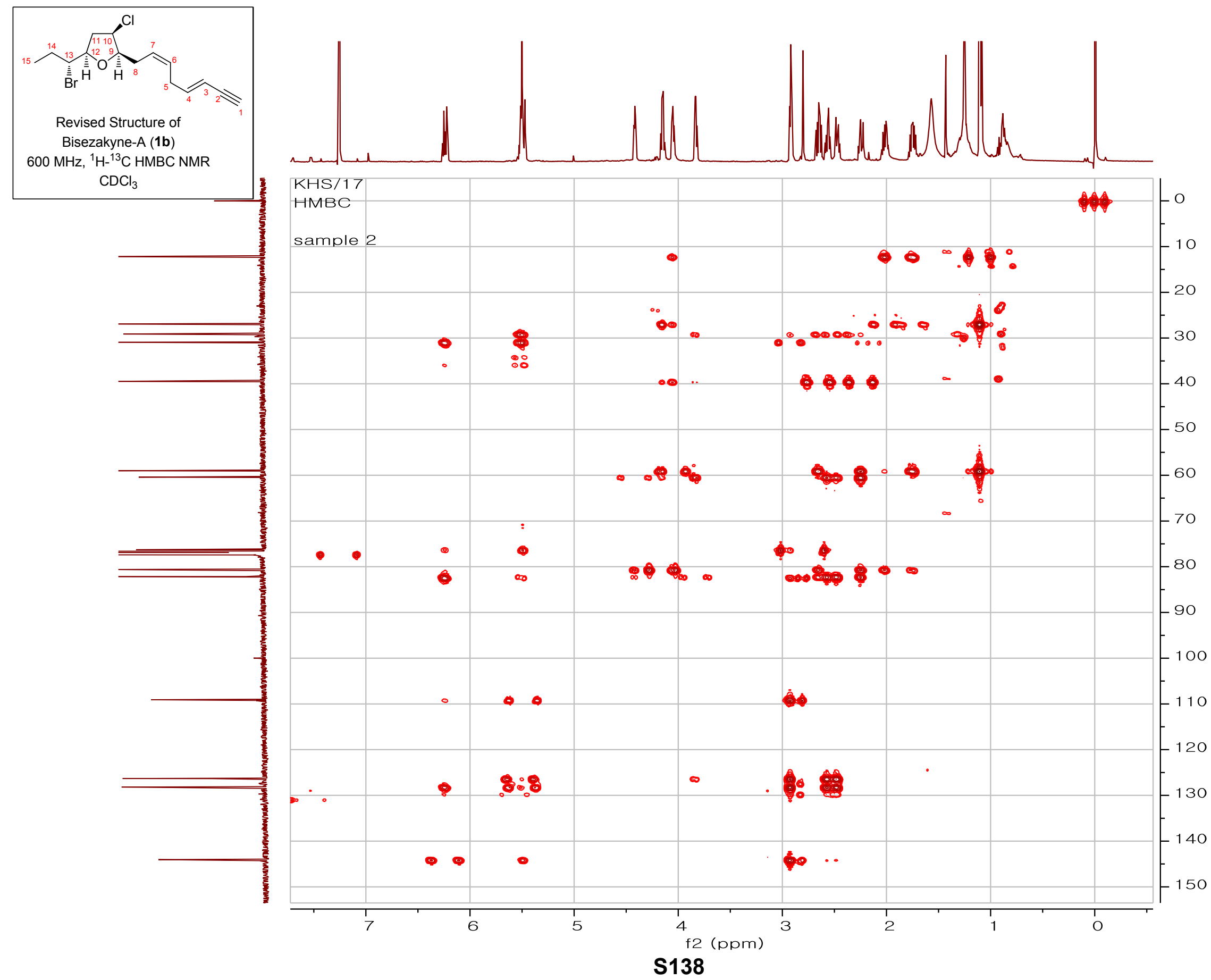



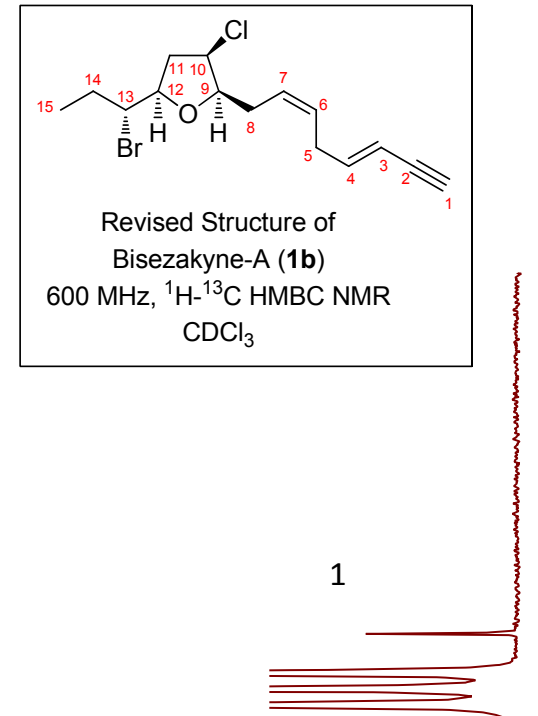

12

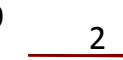

NIN

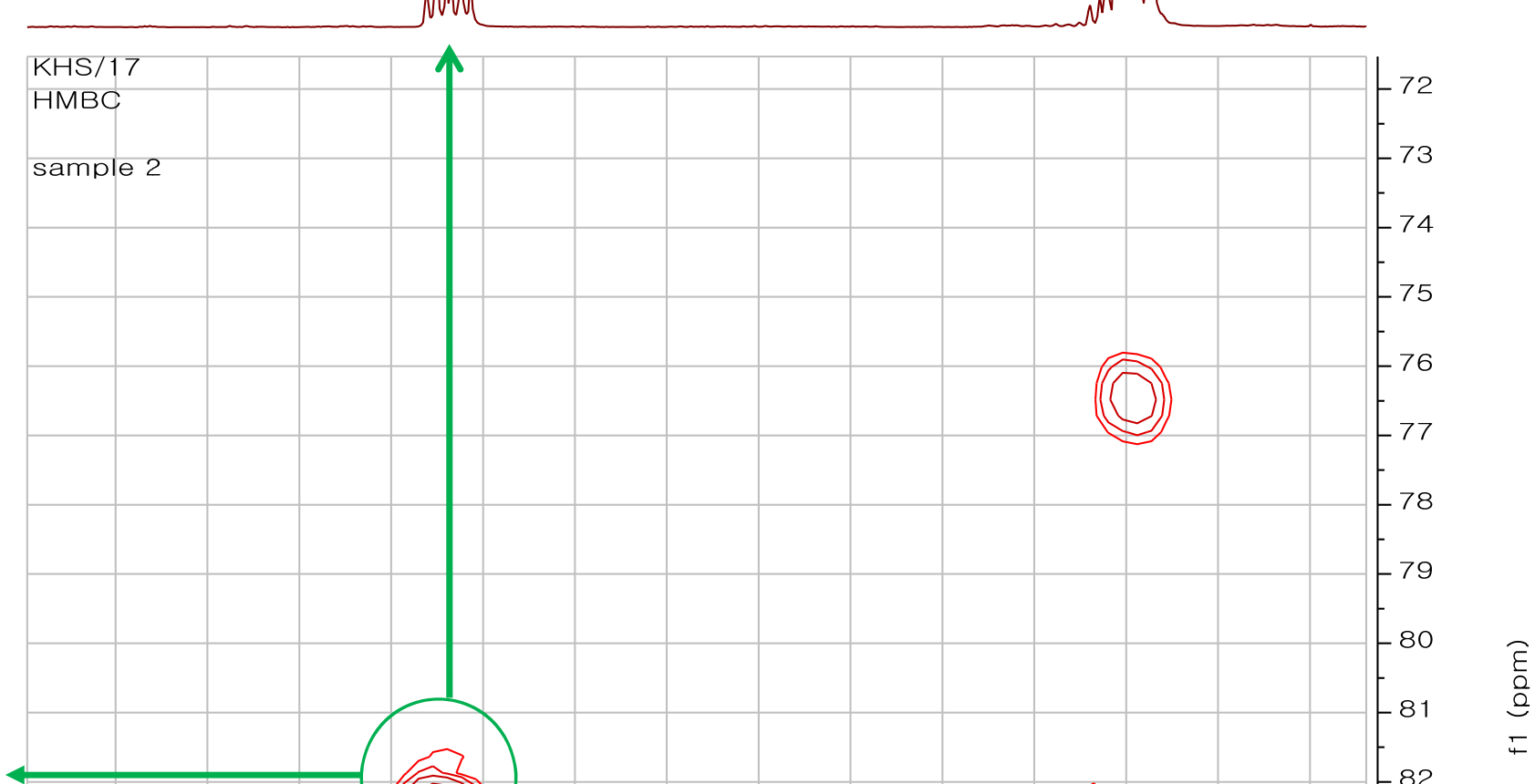

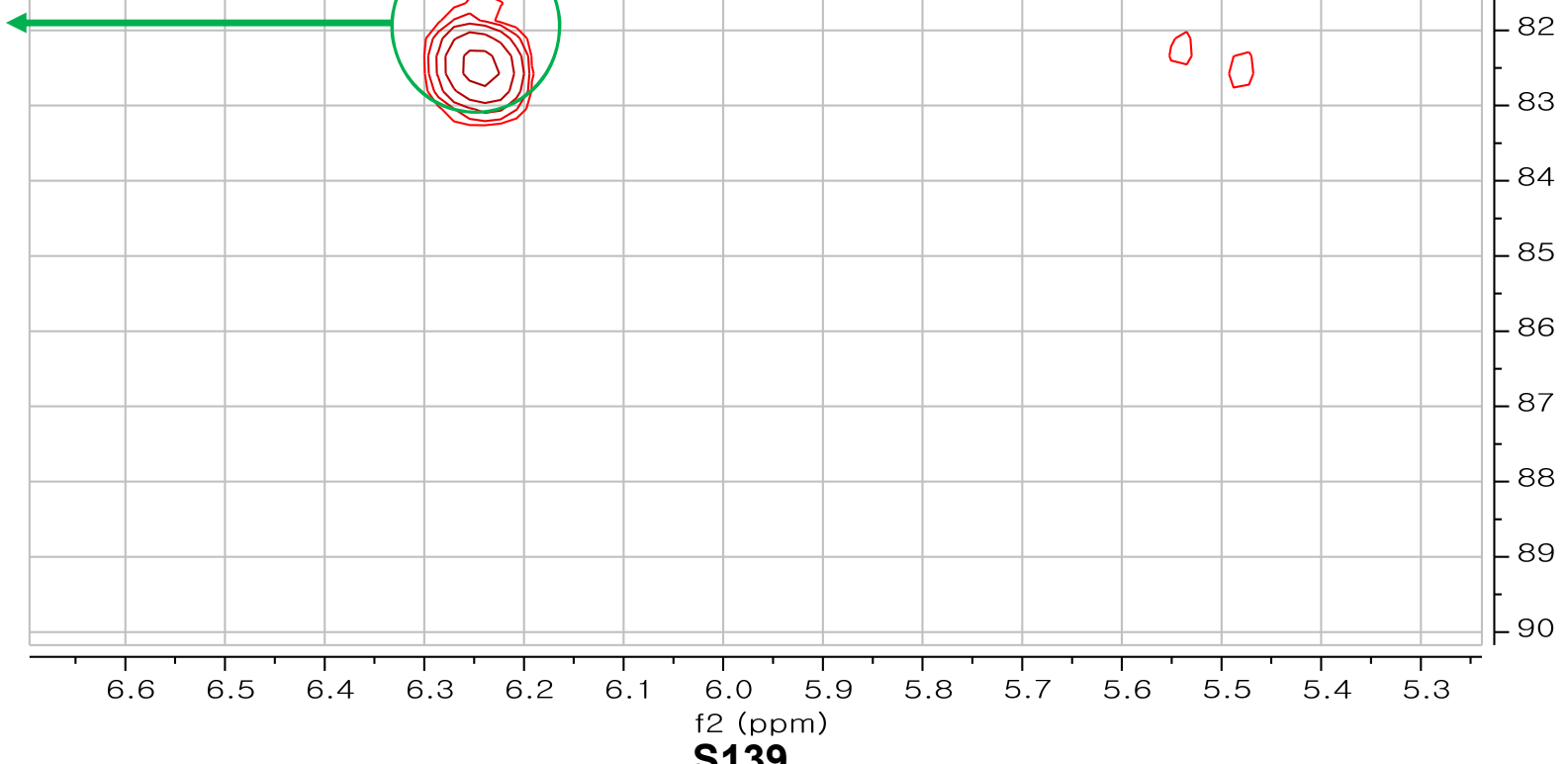




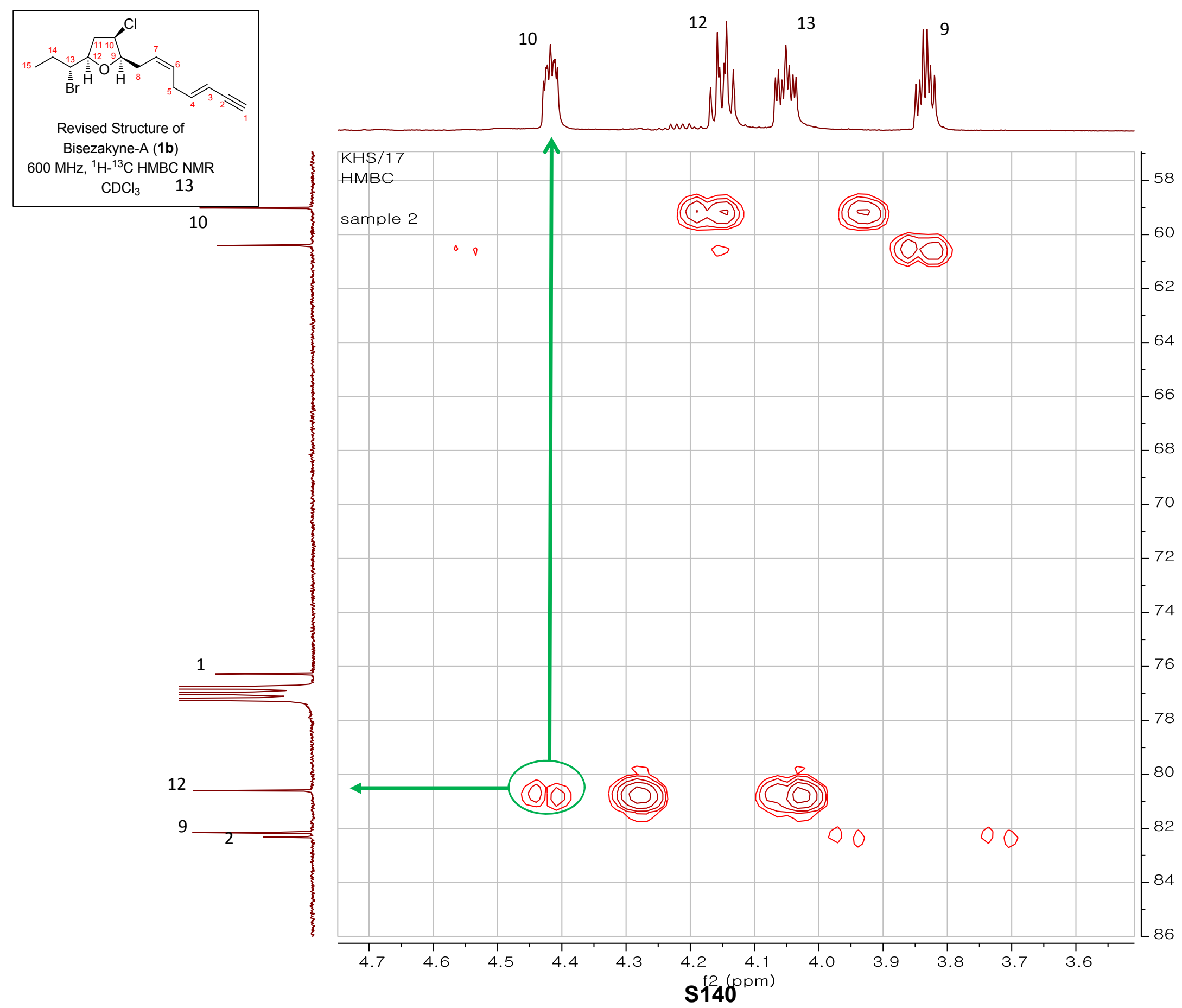

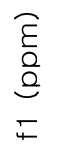




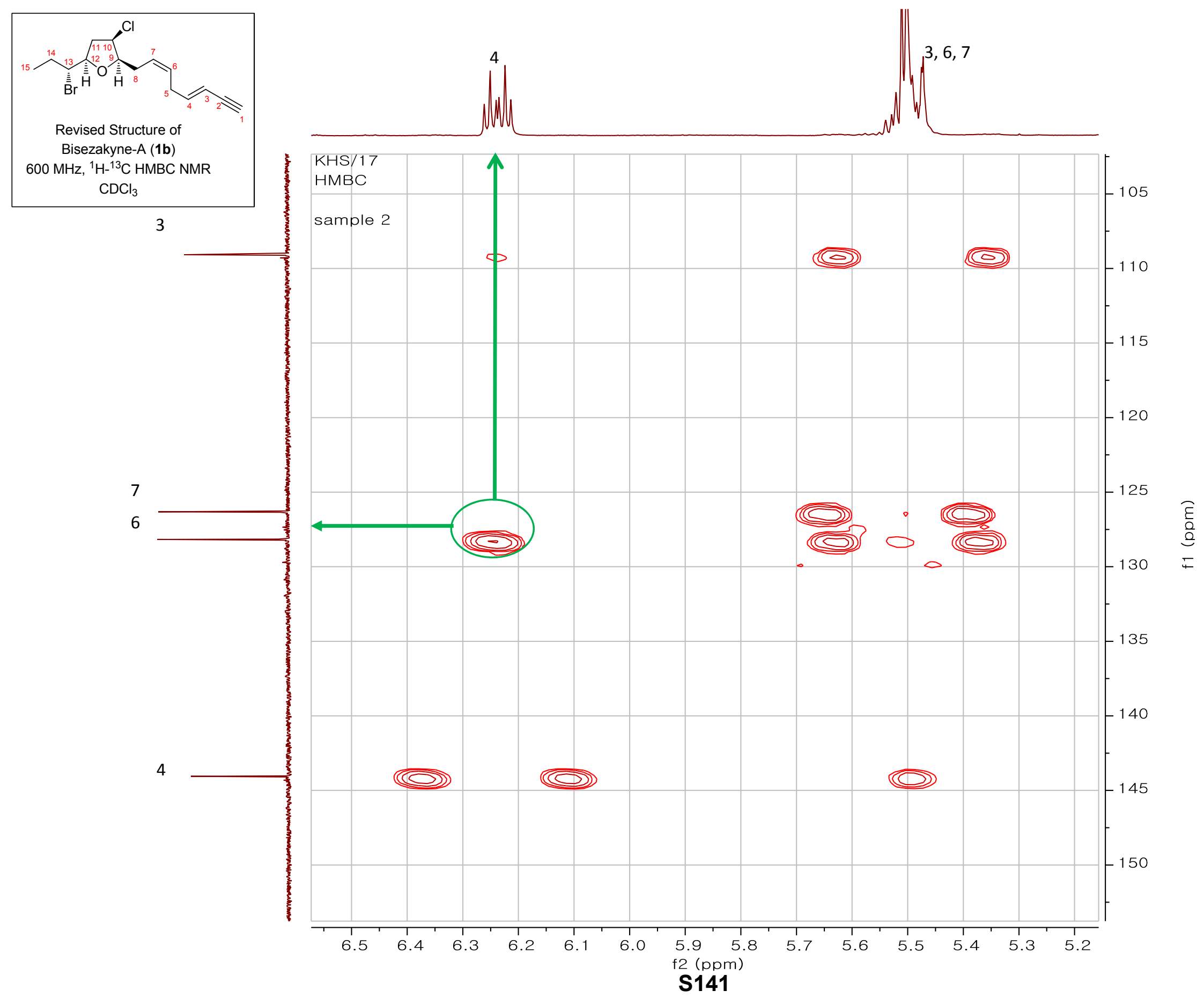




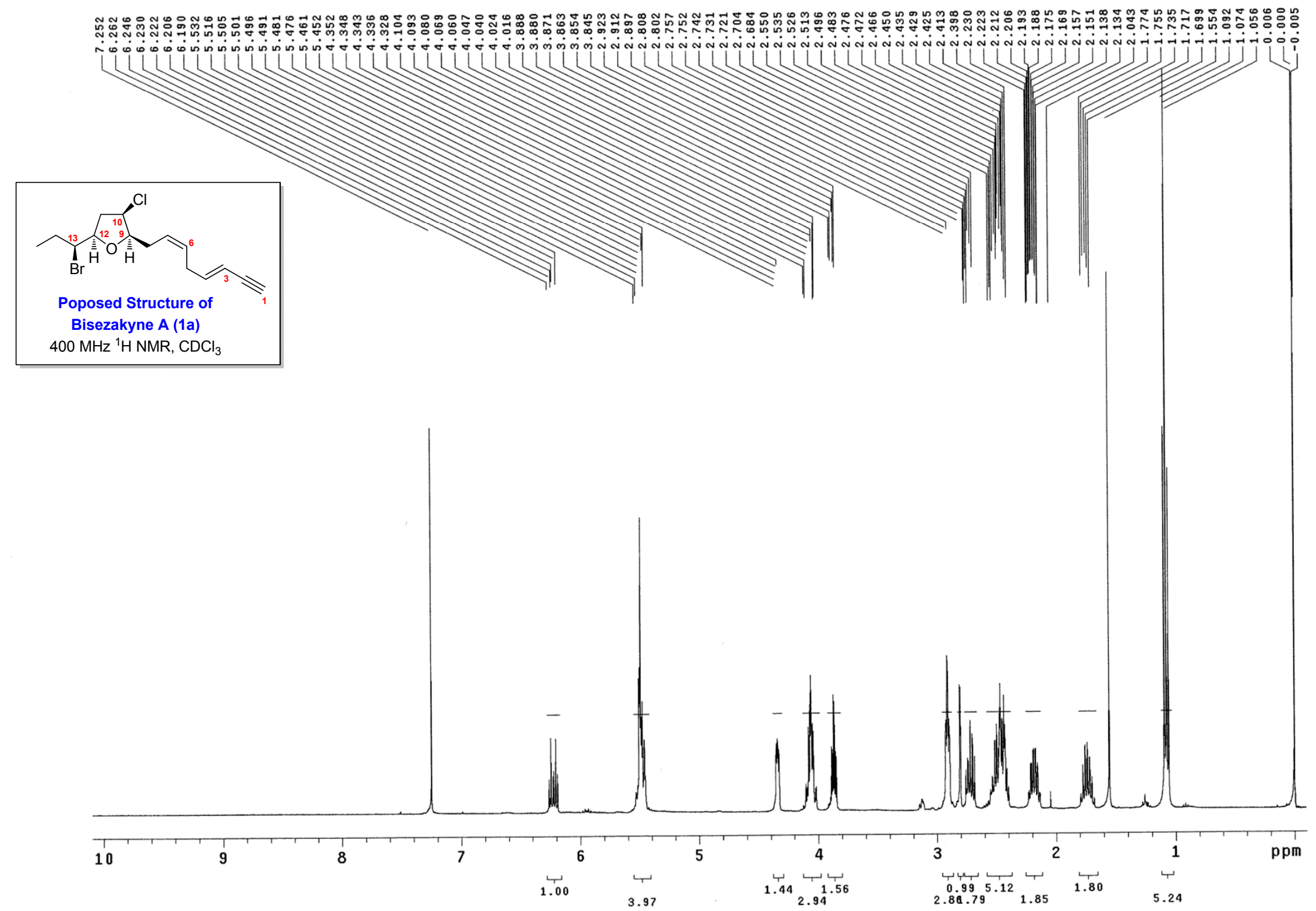

$\mathbf{S 1 4 2}$ 


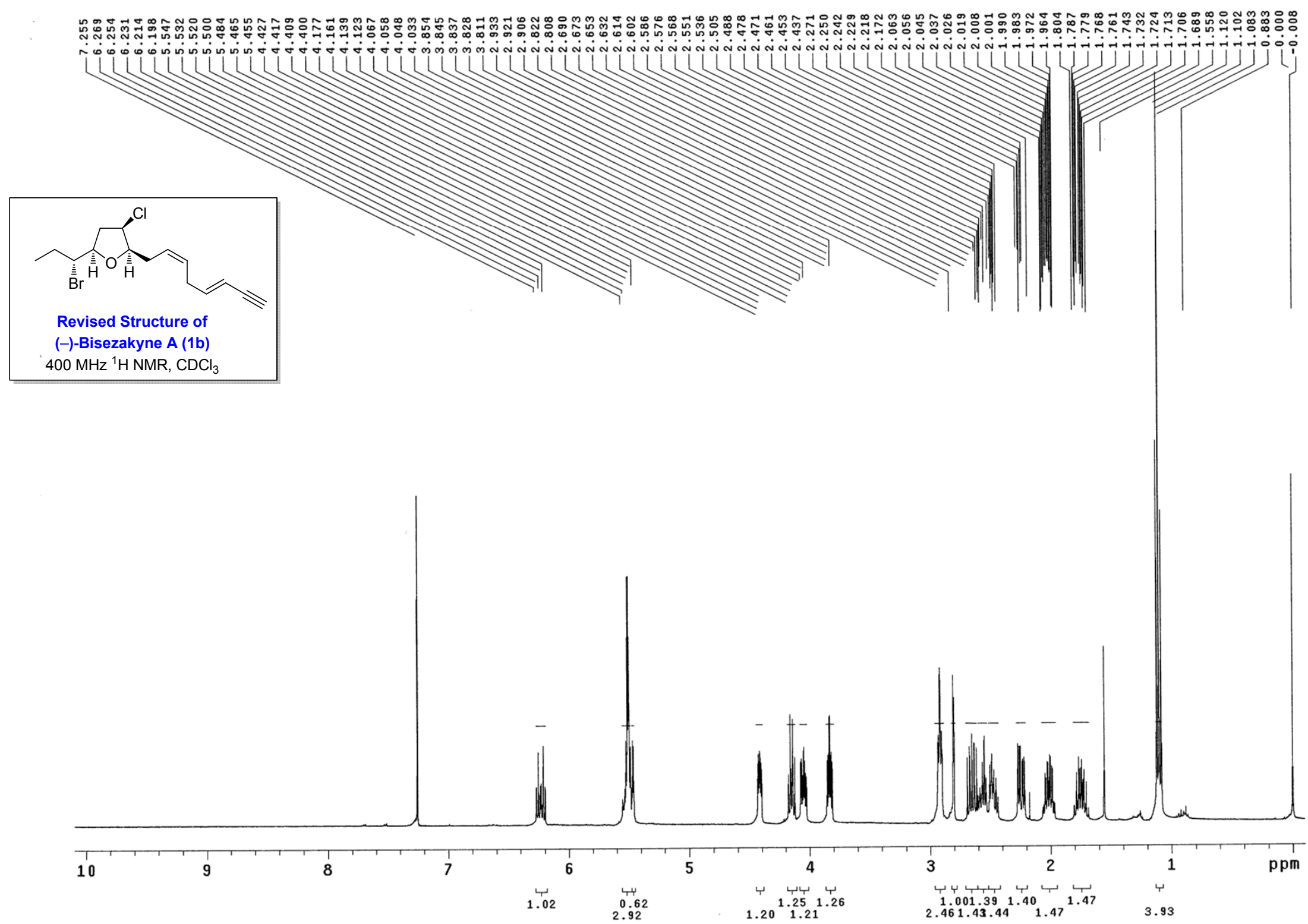

S143 


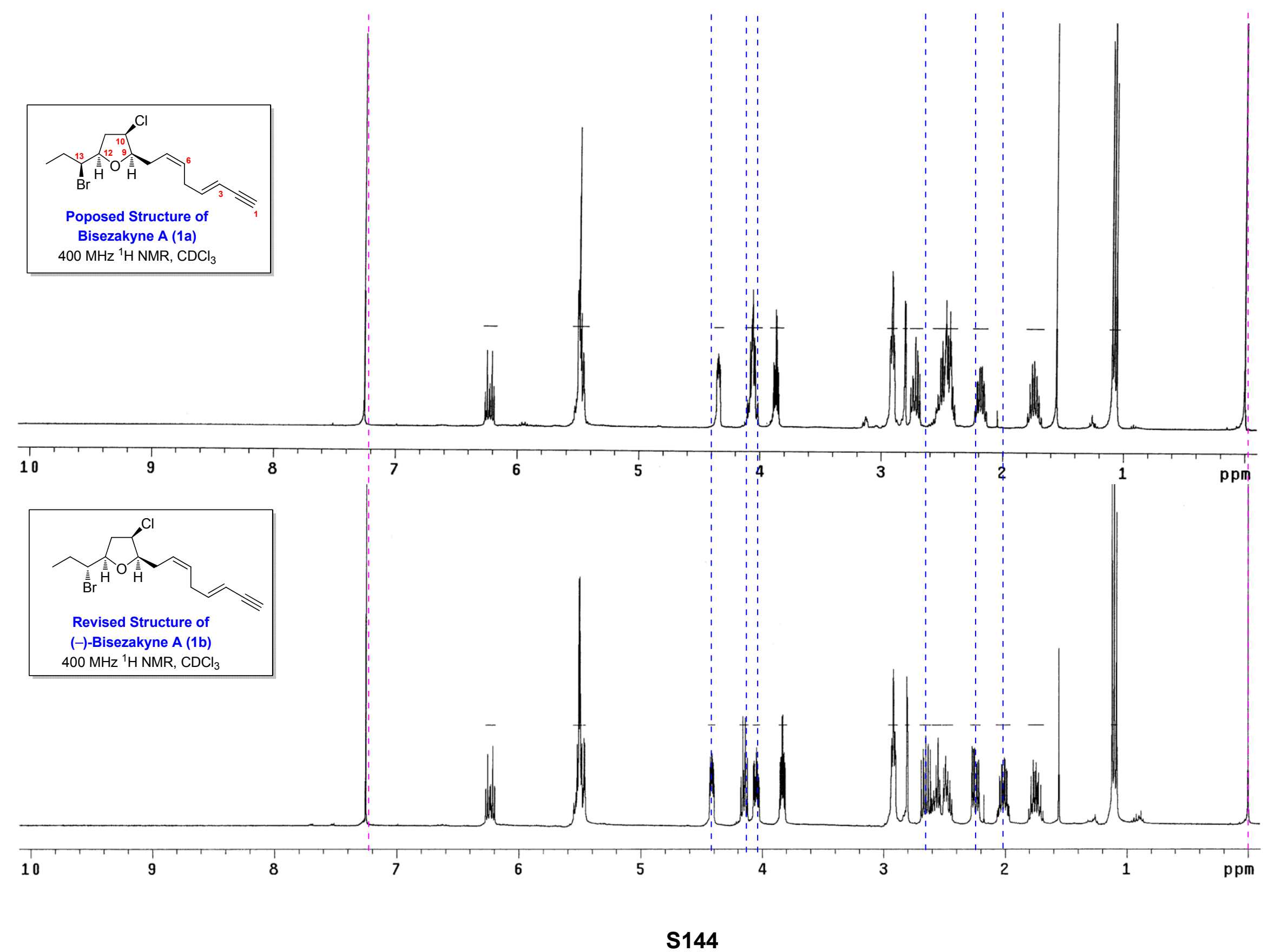




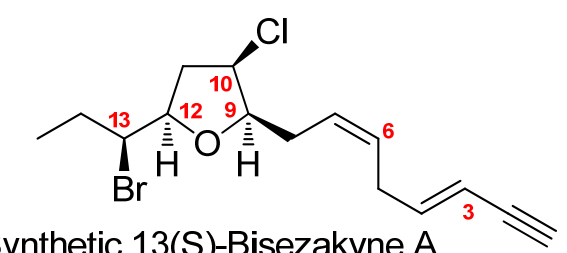

Synthetic 13(S)-Bisezakyne A

$$
\text { (erythro) }
$$

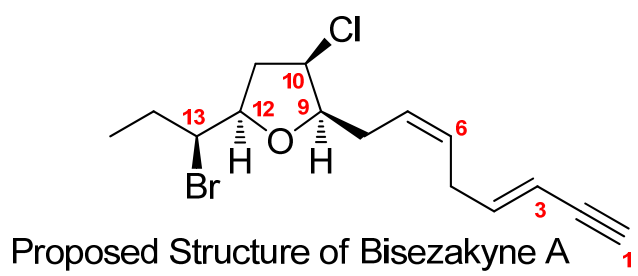

(erythro) By Suzuki Group

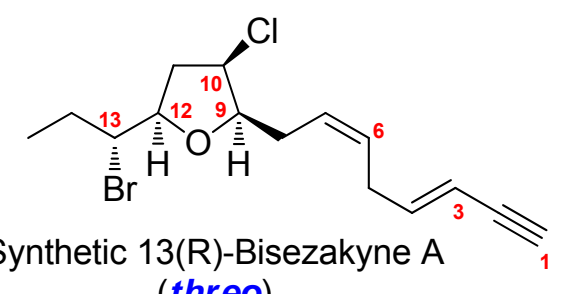

(threo)

\begin{tabular}{|c|c|}
\hline $\begin{array}{l}\text { Carbon } \\
\text { number }\end{array}$ & Synthetic 13(S) (erythro, Proposed) \\
\hline 1 & $2.80(d, J=2.4)$ \\
\hline \multicolumn{2}{|l|}{2} \\
\hline 3 & 5.53-5.45 (m) \\
\hline 4 & 6.23 (ddd, $J=16.4,6.8,6.8$ ) \\
\hline 5 & $2.91(d d, J=6.0,2.0)$ \\
\hline 6 & $5.53-5.45(\mathrm{~m})$ \\
\hline 7 & $5.53-5.45(\mathrm{~m})$ \\
\hline \multirow[t]{2}{*}{8} & $2.51(\mathrm{~m})$ \\
\hline & $2.47(\mathrm{~m})$ \\
\hline 9 & 3.87 (ddd, $J=6.8,6.8,3.6$ ) \\
\hline 10 & 4.34 (ddd, J=6.0, 3.2, 1.6) \\
\hline \multirow[t]{2}{*}{11} & 2.72 (ddd, J= 14.4, 8.4, 6.0) \\
\hline & $2.43(\mathrm{~m})$ \\
\hline 12 & 4.07 (ddd, J=9.2, 9.2, 4.4) \\
\hline 13 & 4.04 (ddd, J= 9.6, 9.6, 2.8) \\
\hline \multirow[t]{2}{*}{14} & 2.18 (ddddd, J= 14.8, 7.6, 7.6, 7.6 2.8) \\
\hline & 1.74 (ddddd, J= 14.8, 8.4, 7.6, 7.6, 7.6) \\
\hline 15 & $1.07(d d, J=7.2,7.2)$ \\
\hline
\end{tabular}

Natural

Synthetic 13(R) (threo, Revised)

$2.89(d, J=1.5)$

$2.81(d, J=1.6)$

5.49 (br dd, J=16.1, 6.4, 6.4)

5.48 (dddd, $\mathrm{J}=16.0,2.0,2.0,2.0$ )

6.24 (ddd, J= 16.1, 6.4, 6.4)

6.23 (ddd, $J=16.0,6.0,6.0$ )

$2.92(\mathrm{~m})$

$5.51(\mathrm{~m})$

$5.51(\mathrm{~m})$

2.56 (ddd, J= 13.7, 6.4, 6.4; $\mathrm{Ha}$ )

2.47 (ddd, J= 13.7, 6.8, 6.8; $\mathrm{Hb}$ )

3.83 (ddd, $J=6.8,6.4,3.6$ )

4.42 (ddd, $J=6.8,3.6,2.4$ )

2.65 (ddd, J= 15.1, 8.3, 6.8; Ha)

2.24 (ddd, J= 15.1, 6.4, 2.4; $\mathrm{Hb}$ )

4.15 (ddd, $J=8.3,6.4,6.4$ )

4.05 (ddd, J= 9.9, 6.4, 2.9)

2.01 (ddddd, $\mathrm{J}=14.6,7.3,7.3,7.3,2.9$ )

2.92 (ddd, $J=6.0,6.0,1.2$ )

5.55-5.48 (m)

5.55-5.48 (m)

2.57 (ddd, , J = 12.8, 6.0, 6.0)

2.47 (ddd, , $J=13.6,6.8,6.8$ )

3.83 (ddd, $J=6.8,6.8,3.6$ )

4.42 (ddd, J = 6.8, 3.6, 2.0)

2.65 (ddd, J = 14.8, 8.4, 6.8)

2.24 (ddd, $J=15.2,6.4,2.4$ )

4.15 (ddd, $J=8.4,6.4,6.4$ )

4.05 (ddd, $\mathrm{J}=10.0,6.0,2.8$ )

2.01 (ddddd, $\mathrm{J}=14.8,7.2,7.2,7.2,2.8$ )

1.75 (ddddd, $\mathrm{J}=14.4,10.0,6.8,6.8,6.8$ )

1.10 (dd, $\mathrm{J}=7.2,7.2$ ) 


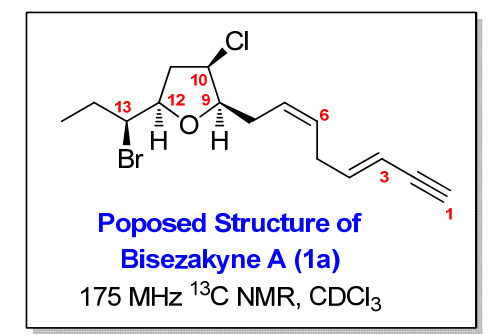

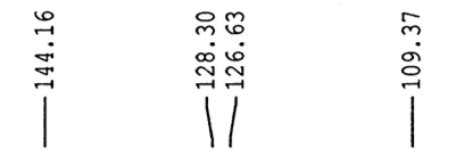

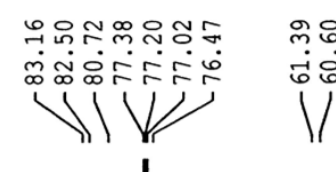

|

||
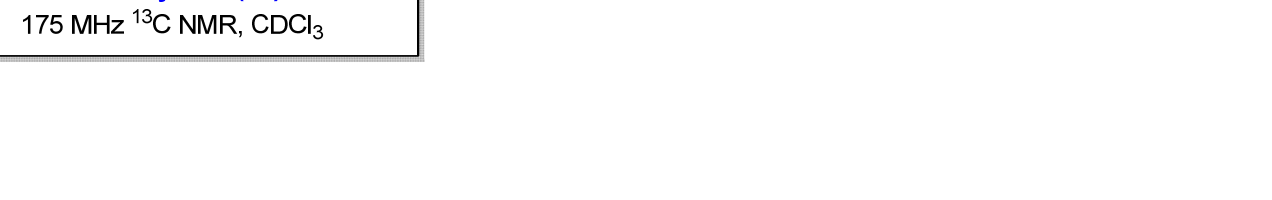

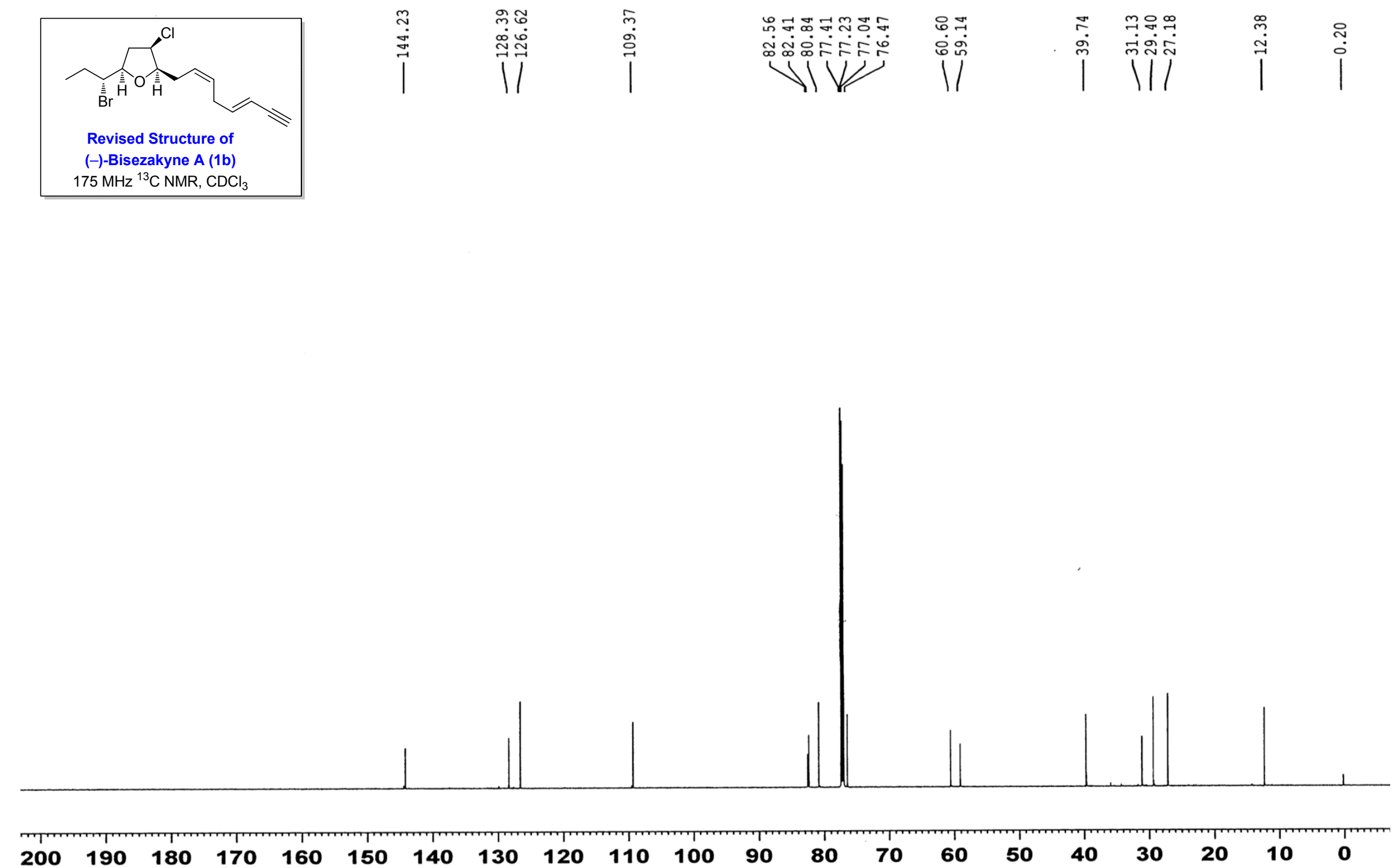

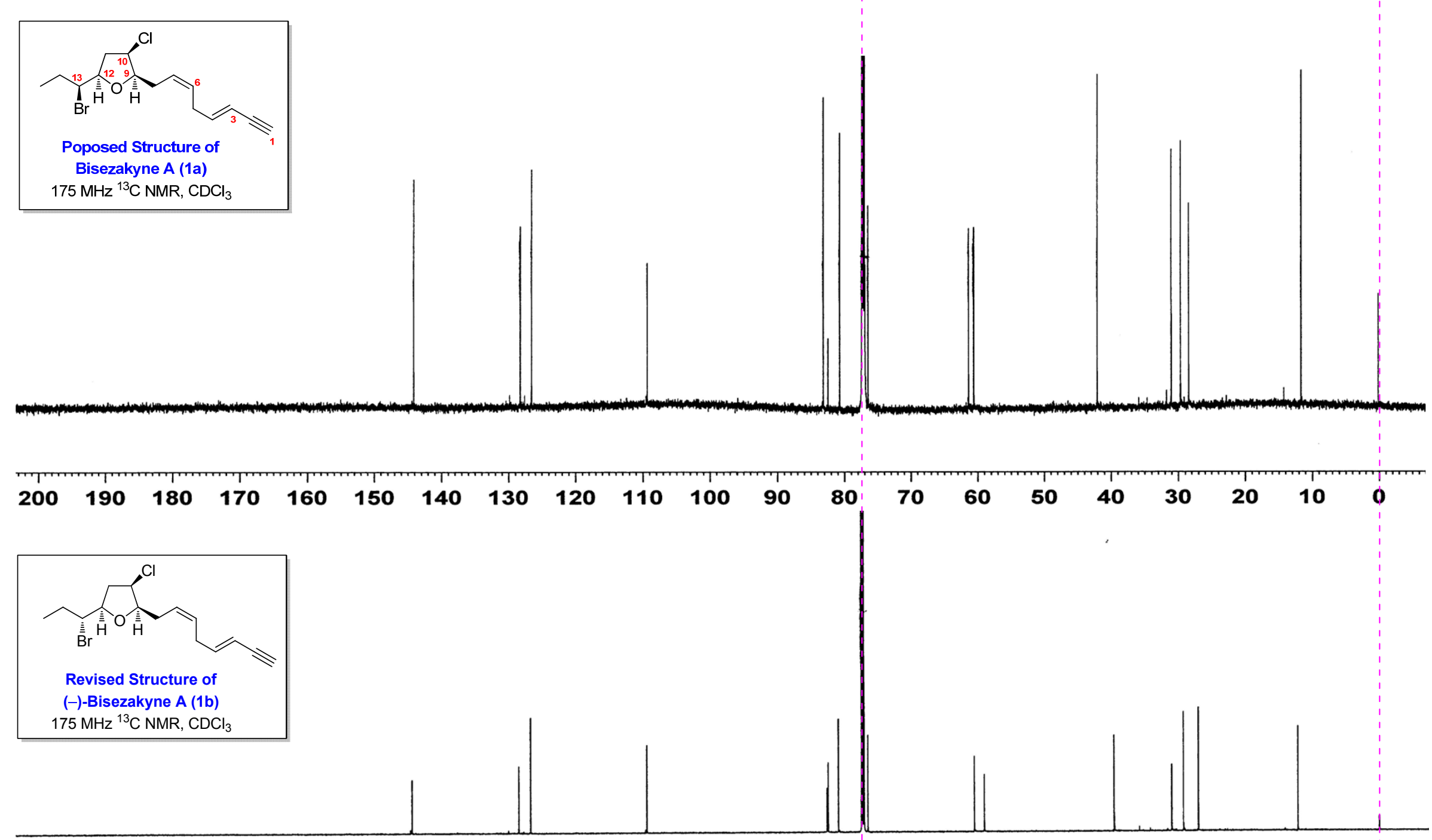

$\begin{array}{lllllllllllllllllllll}200 & 190 & 180 & 170 & 160 & 150 & 140 & 130 & 120 & 110 & 100 & 90 & 80 & 70 & 60 & 50 & 40 & 30 & 20 & 10 & 0\end{array}$ 


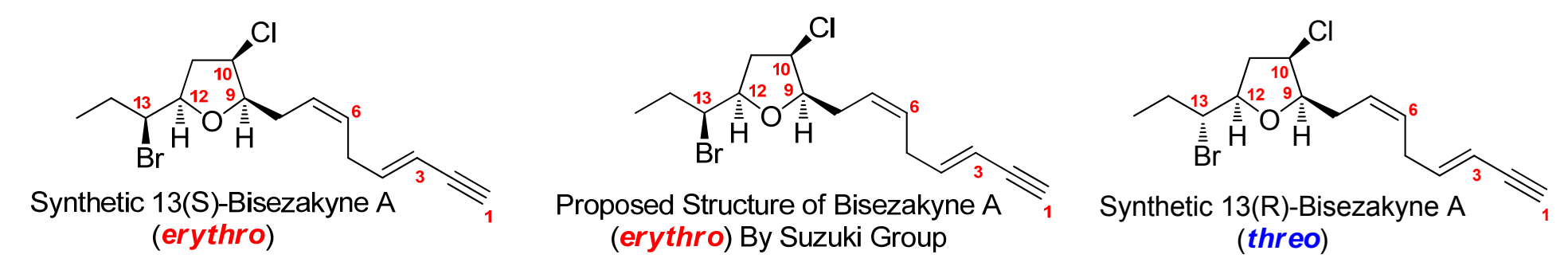

\begin{tabular}{|c|c|c|c|c|c|}
\hline $\begin{array}{l}\text { Carbon } \\
\text { number }\end{array}$ & $\begin{array}{l}\text { Synthetic 13(S) } \\
\text { (Proposed) }\end{array}$ & $\begin{array}{c}\Delta \\
\text { Syn.(erythro)-Nat. }\end{array}$ & Natural & $\begin{array}{c}\Delta \\
\text { Syn.(threo)-Nat. }\end{array}$ & $\begin{array}{c}\text { Synthetic 13(R) } \\
\text { (Revised) }\end{array}$ \\
\hline 1 & 76.3 & 0.0 & 76.3 & -0.1 & 76.2 \\
\hline 2 & 83.0 & 0.6 & 82.4 & -0.1 & 82.3 \\
\hline 3 & 109.2 & 0.1 & 109.1 & 0.0 & 109.1 \\
\hline 4 & 144.0 & 0.0 & 144.0 & 0.0 & 144.0 \\
\hline 5 & 30.9 & 0.0 & 30.9 & 0.0 & 30.9 \\
\hline 6 & 128.1 & -0.1 & 128.2 & 0.0 & 128.2 \\
\hline 7 & 126.4 & 0.0 & 126.4 & 0.0 & 126.4 \\
\hline 8 & 29.5 & 0.3 & 29.2 & 0.0 & 29.2 \\
\hline 9 & 82.3 & 0.1 & 82.2 & 0.0 & 82.2 \\
\hline 10 & 61.2 & 0.8 & 60.4 & 0.0 & 60.4 \\
\hline 11 & 41.9 & 2.4 & 39.5 & 0.0 & 39.5 \\
\hline 12 & 80.5 & -0.1 & 80.6 & 0.0 & 80.6 \\
\hline 13 & 60.4 & 1.4 & 59.0 & -0.1 & 58.9 \\
\hline 14 & 28.3 & 1.3 & 27.0 & 0.0 & 27.0 \\
\hline 15 & 11.5 & -0.7 & 12.2 & 0.0 & 12.2 \\
\hline
\end{tabular}

